\title{
Measuring atmospheric composition change
}

P. Laj ${ }^{\text {a,b,* }}$, J. Klausen ${ }^{c}$, M. Bilde ${ }^{\text {d }}$, C. Plaß-Duelmer ${ }^{\mathrm{e}}$, G. Pappalardo ${ }^{\mathrm{f}}$, C. Clerbaux $^{\mathrm{g}, 1}$, U. Baltensperger ${ }^{\mathrm{h}}$, J. Hjorth $^{\mathrm{i}}$, D. Simpson ${ }^{\mathrm{j}, \mathrm{k}}$, S. Reimann ${ }^{\mathrm{c}}$, P.-F. Coheur ${ }^{1}$, A. Richter ${ }^{\mathrm{m}}$, M. De Mazière ${ }^{\mathrm{n}}$, Y. Rudich $^{\mathrm{o}}$, G. McFiggans $^{\mathrm{p}}$, K. Torseth ${ }^{\mathrm{q}}$, A. Wiedensohler ${ }^{\mathrm{r}}$, S. Morin ${ }^{\mathrm{b}, 1}$, M. Schulz ${ }^{\mathrm{s}}$, J.D. Allan ${ }^{\mathrm{p}}$, J.-L. Attié ${ }^{\mathrm{t}}$, I. Barnes ${ }^{\mathrm{u}}$, W. Birmili ${ }^{\mathrm{r}}$, J.P. Cammas ${ }^{\mathrm{t}}$, J. Dommen ${ }^{\mathrm{h}}$, H.-P. Dorn ${ }^{\mathrm{w}}$, D. Fowler ${ }^{\mathrm{x}}$, S. Fuzzi ${ }^{\mathrm{v}}$, M. Glasius ${ }^{d}$, C. Granier ${ }^{\text {g,y,z }}$, M. Hermann ${ }^{\mathrm{r}}$, I. Isaksen ${ }^{\mathrm{aa}, \mathrm{bb}}$, S. Kinne ${ }^{\mathrm{cc}}$, I. Koren ${ }^{\mathrm{o}}$, F. Madonna ${ }^{\mathrm{f}}$, M. Maione ${ }^{\mathrm{dd}}$, A. Massling ee , O. Moehler ff, L. Mona ${ }^{\text {f, P.S. Monks }}{ }^{\text {gg }}$, D. Müller ${ }^{\text {r, hh }}$, T. Müller ${ }^{\mathrm{r}}$, J. Orphal ${ }^{\mathrm{ii}, 2}$, V.-H. Peuch ${ }^{\mathrm{jj}}$, F. Stratmann ${ }^{\mathrm{r}}$, D. Tanré ${ }^{\mathrm{kk}}$, G. Tyndall $^{\mathrm{ll}}$, A. Abo Riziq ${ }^{\mathrm{mm}}$,

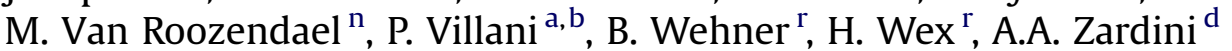

${ }^{a}$ Laboratoire de Météorologie Physique, Observatoire de Physique du Globe de Clermont-Ferrand, Université Blaise Pascal - CNRS, 63177 Aubière, France

${ }^{\mathrm{b}}$ Université Joseph Fourier - Grenoble 1 / CNRS, Laboratoire de Glaciologie et Géophysique de l'Environnement, 38400 St Martin d'Hères, France

${ }^{\mathrm{c}}$ Empa, Swiss Federal Laboratories for Materials Testing and Research, Laboratory for Air Pollution/Environmental Technology, 8600 Dübendorf, Switzerland

${ }^{\mathrm{d}}$ Department of Chemistry, University of Copenhagen, 2100 Copenhagen, Denmark

e Deutscher Wetterdienst, Meteorologisches Observatorium Hohenpeissenberg, 82383 Hohenpeissenberg, Germany

${ }^{\mathrm{f}}$ Istituto di Metodologie per l'Analisi Ambientale - CNR, 85050 Tito Scalo, Italy

${ }^{g}$ UPMC Univ. Paris 06; Université Versailles St.-Quentin; CNRS/INSU, LATMOS-IPSL, 75005 Paris, France

${ }^{\text {h }}$ Paul Scherrer Institut, Laboratory of Atmospheric Chemistry, 5232 Villigen, Switzerland

i Institute for Environment and Sustainability, The European Commission, Joint Research Centre, 21020 Ispra, Italy

${ }^{\mathrm{j}}$ EMEP MSC-W, Norwegian Meteorological Institute, 0313 Oslo, Norway

${ }^{\mathrm{k}}$ Department of Radio and Space Science, Chalmers University of Technology, 41296 Gothenburg, Sweden

${ }^{1}$ Spectroscopie de l'Atmosphère, Service de Chimie Quantique et Photophysique, Université Libre de Bruxelles, 1050 Brussels, Belgium

${ }^{\mathrm{m}}$ Institute of Environmental Physics, University of Bremen, 28359 Bremen, Germany

${ }^{\mathrm{n}}$ Belgian Institute for Space Aeronomy, 1180 Brussels, Belgium

${ }^{\circ}$ Department of Environmental Sciences and Energy Research, Weizmann Institute of Science, 76100 Rehovot, Israel

${ }^{\mathrm{p}}$ School of Earth, Atmospheric and Environmental Sciences, The University of Manchester, M13 9PL Manchester, United Kingdom

${ }^{\mathrm{q}}$ Department of Regional and Global Pollution Issues, Norwegian Institute for Air Research, 2027 Kjeller, Norway

${ }^{\mathrm{r}}$ Leibniz Institute for Tropospheric Research, 04318 Leipzig, Germany

${ }^{s}$ Laboratoire des Sciences du Climat et de l'Environnement, CNRS - CEA, 91190 Gif sur Yvette, France

${ }^{\mathrm{t}}$ Laboratoire d'Aérologie, CNRS - Université de Toulouse, 31400, Toulouse, France

${ }^{u}$ Bergische Universitaet Wuppertal, Fachbereich C Physikalische Chemie, 42119 Wuppertal, Germany

${ }^{v}$ Istituto di Scienze dell'Amtosfera e del Clima - CNR, 40129 Bologna, Italy

${ }^{\mathrm{w}}$ Institut für Chemie und Dynamik der Geosphäre, ICG-II: Troposphere, Forschungszentrum Jülich, 52425 Jülich, Germany

${ }^{x}$ Centre of Ecology and Hydrology, EH26 OQB Penicuik Midlothian, UK

${ }^{\mathrm{y}}$ NOAA Earth System Research Laboratory, 80305-3337 Boulder, USA

${ }^{2}$ Cooperative Institute for Research in Environmental Sciences, University of Colorado, 80309-0216 Boulder, USA

${ }^{\text {aa }}$ Department of Geosciences, University of Oslo, 0316 Oslo, Norway

${ }^{\mathrm{bb}}$ Center for International Climate and Environmental Research - Oslo, 0318 Oslo, Norway

${ }^{\mathrm{cc}}$ Max-Planck-Institut für Meteorologie, 20146 Hamburg, Germany

dd Universita' di Urbino, Istituto di Scienze Chimiche “F. Bruner”, 61029 Urbino, Italy

ee National Environmental Research Institute, Department of Atmospheric Environment, Aarhus University, 4000 Roskilde, Denmark

${ }^{\mathrm{ff}}$ Atmospheric Aerosol Research Department, Institute of Meteorology and Climate Research (IMK), Research Centre Karlsruhe, 76021 Karlsruhe, Germany

${ }^{g g}$ Department of Chemistry, University of Leicester, LE1 7RH Leicester, UK

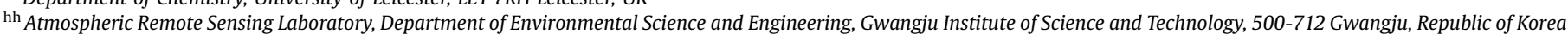

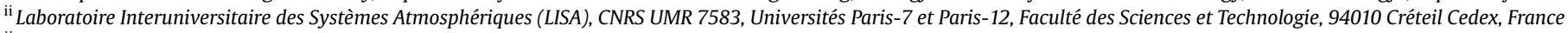

${ }^{\mathrm{jj}}$ Meteo-France, CNRM/GMGEC/CARMA, 31057 Toulouse, France

${ }_{\mathrm{kk}}$ Laboratoire d'Optique Atmosphérique, Universite de Lille, 59655 Villeneuve d'Ascq, France

${ }^{11}$ National Center for Atmospheric Research, 80305 Boulder, USA

${ }^{\mathrm{mm}}$ Department of Chemistry and Biochemistry, University of California, 93106 - 9510 Santa Barbara, USA

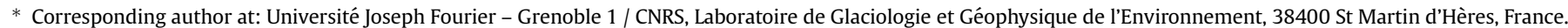
Tel.: +33 4768242 77; fax: +33476 824201 .

E-mail address: Paolo.Laj@lgge.obs.ujf-grenoble.fr (P. Laj).

1 Present address: Météo-France / CNRS, CNRM/GAME, CEN, 38400 St Martin d'Hères, France.

2 Present address: Institut for Meteorology and Climate Research (IMK), Karlsruhe Institute Technology (KIT), Karlsruhe, Germany.
} 


\section{A R T I C L E I N F O}

\section{Article history:}

Received 10 February 2009

Received in revised form

10 July 2009

Accepted 12 August 2009

\section{Keywords:}

Atmosphere

Instrumentation

Observation

Air quality

Climate

\begin{abstract}
A B S T R A C T
Scientific findings from the last decades have clearly highlighted the need for a more comprehensive approach to atmospheric change processes. In fact, observation of atmospheric composition variables has been an important activity of atmospheric research that has developed instrumental tools (advanced analytical techniques) and platforms (instrumented passenger aircrafts, ground-based in situ and remote sensing stations, earth observation satellite instruments) providing essential information on the composition of the atmosphere. The variability of the atmospheric system and the extreme complexity of the atmospheric cycles for short-lived gaseous and aerosol species have led to the development of complex models to interpret observations, test our theoretical understanding of atmospheric chemistry and predict future atmospheric composition. The validation of numerical models requires accurate information concerning the variability of atmospheric composition for targeted species via comparison with observations and measurements.

In this paper, we provide an overview of recent advances in instrumentation and methodologies for measuring atmospheric composition changes from space, aircraft and the surface as well as recent improvements in laboratory techniques that permitted scientific advance in the field of atmospheric chemistry. Emphasis is given to the most promising and innovative technologies that will become operational in the near future to improve knowledge of atmospheric composition. Our current observation capacity, however, is not satisfactory to understand and predict future atmospheric composition changes, in relation to predicted climate warming. Based on the limitation of the current European observing system, we address the major gaps in a second part of the paper to explain why further developments in current observation strategies are still needed to strengthen and optimise an observing system not only capable of responding to the requirements of atmospheric services but also to newly open scientific questions.
\end{abstract}

(C) 2009 Elsevier Ltd. All rights reserved.

\section{Contents}

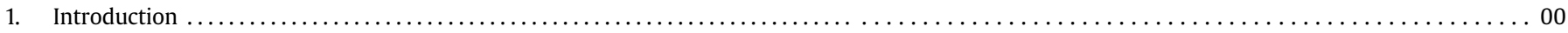

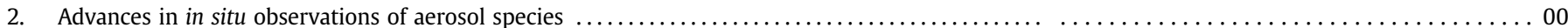

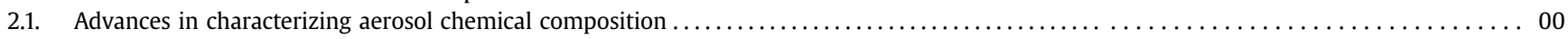

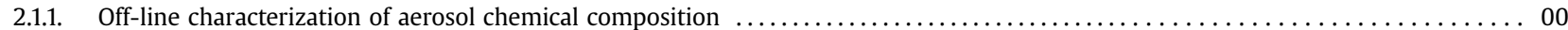

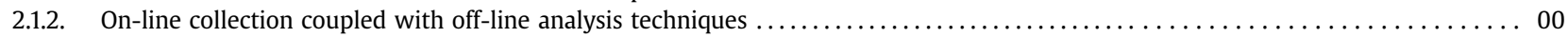

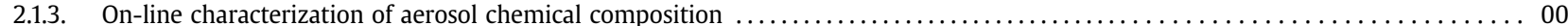

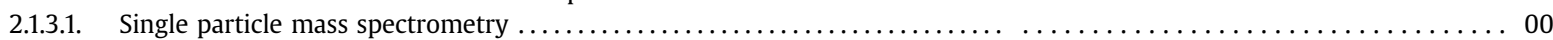

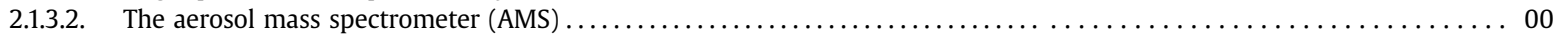

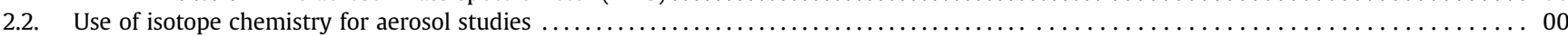

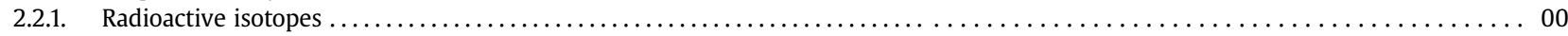

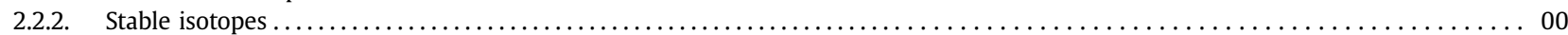

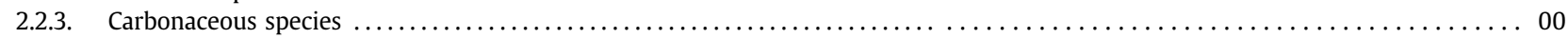

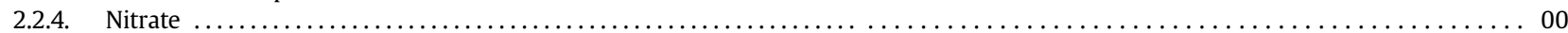

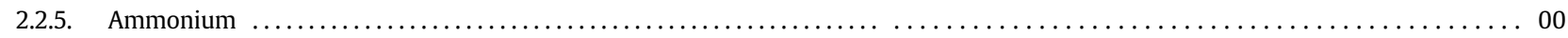

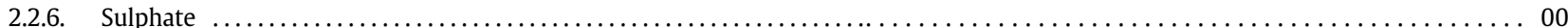

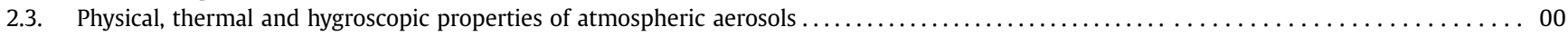

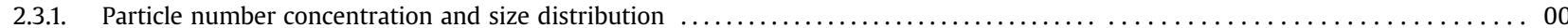

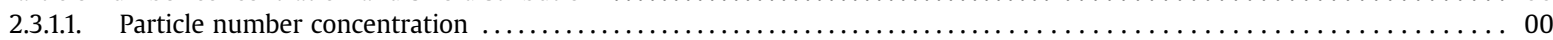

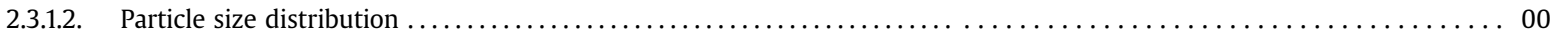

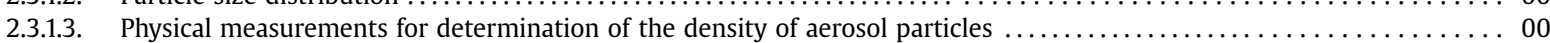

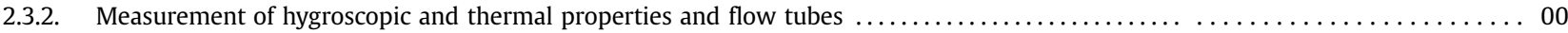

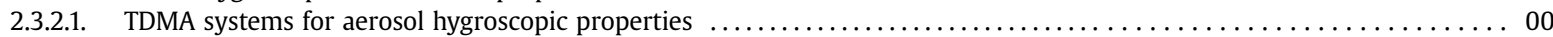

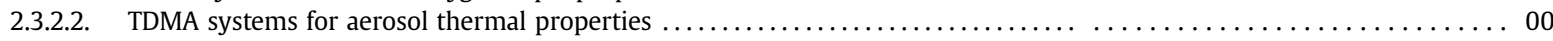

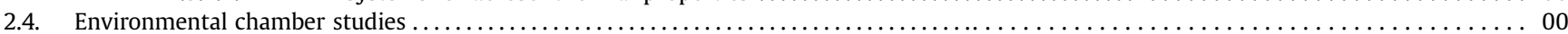

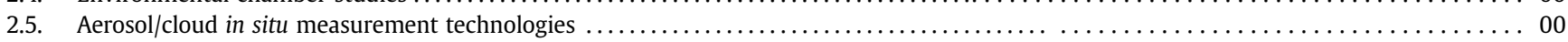

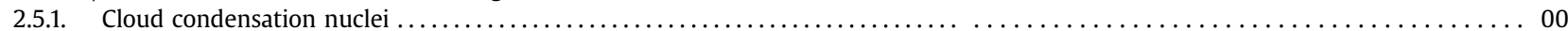

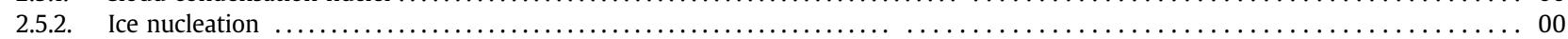

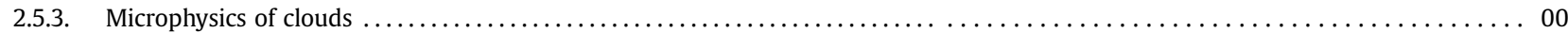

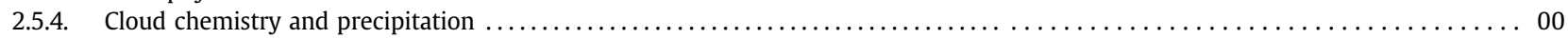

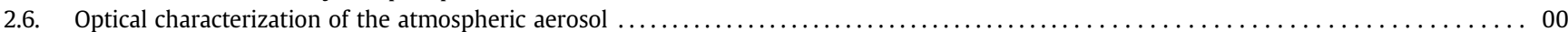

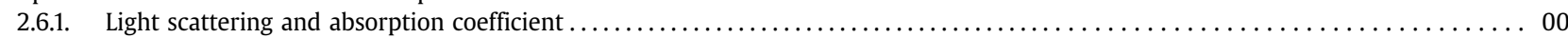

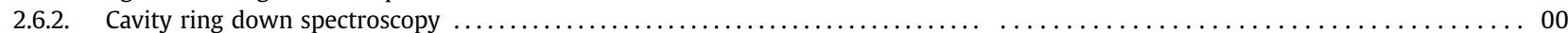

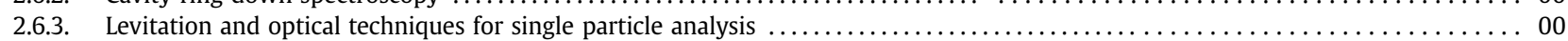

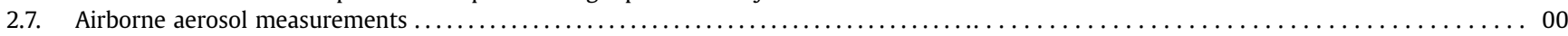

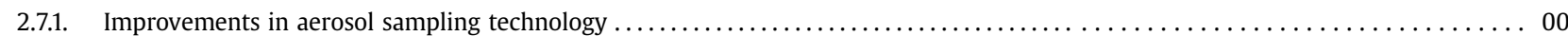

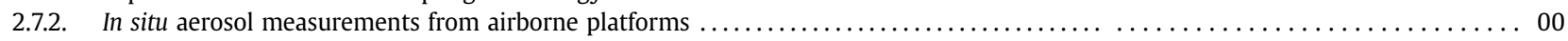

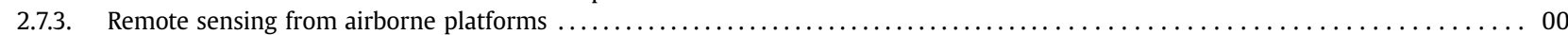

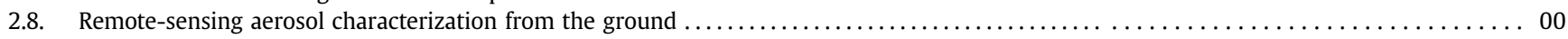

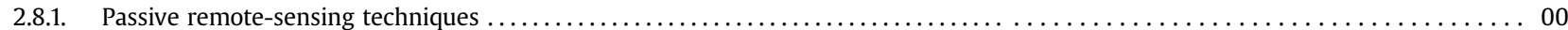

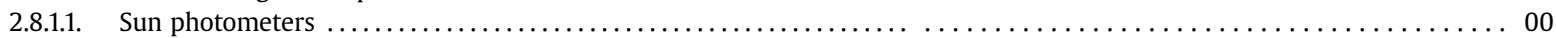




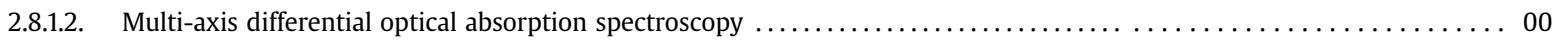

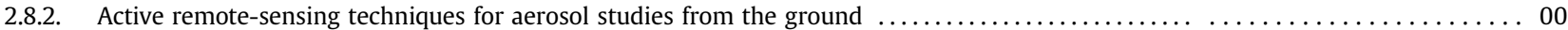

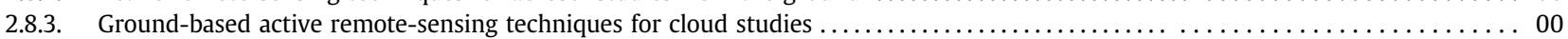

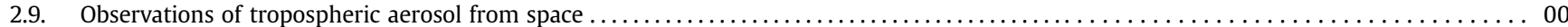

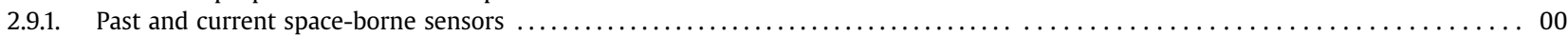

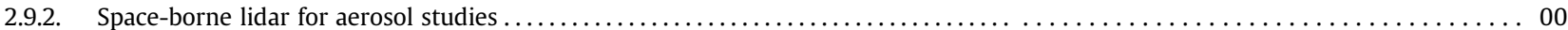

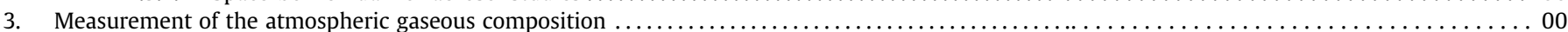

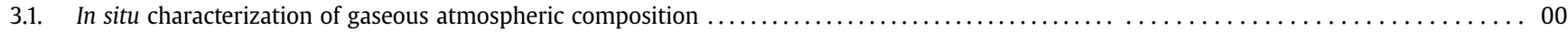

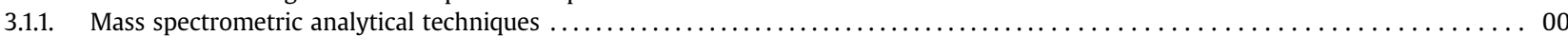

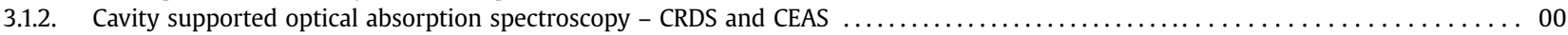

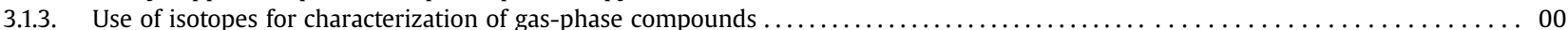

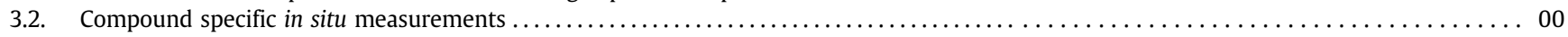

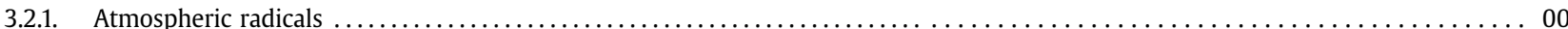

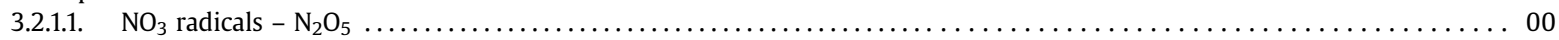

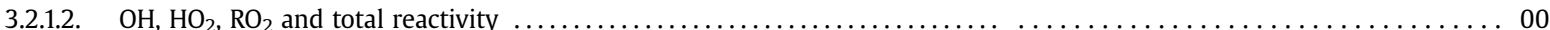

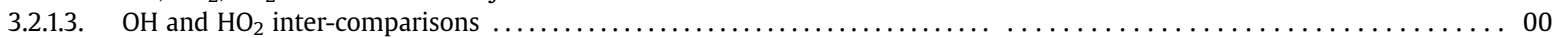

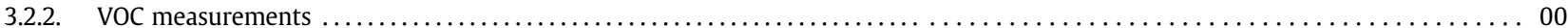

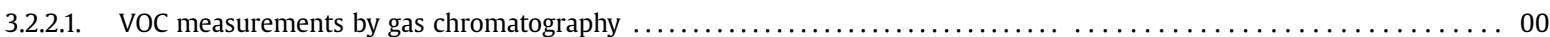

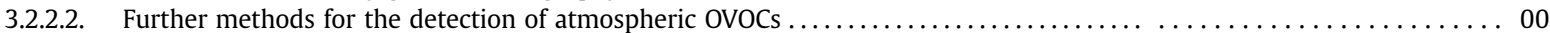

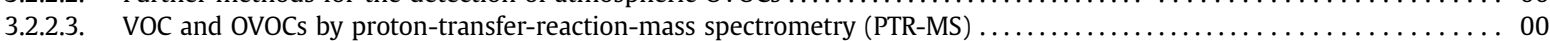

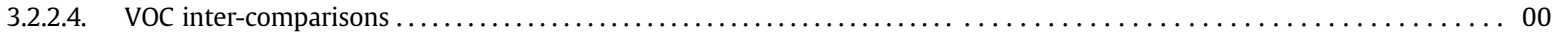

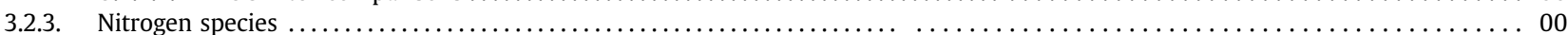

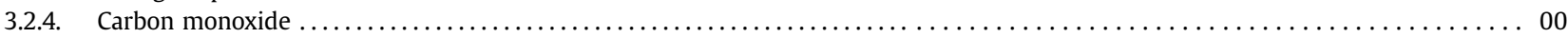

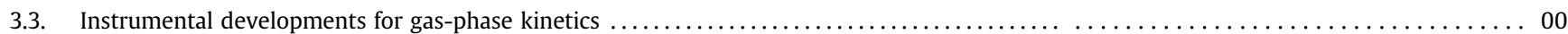

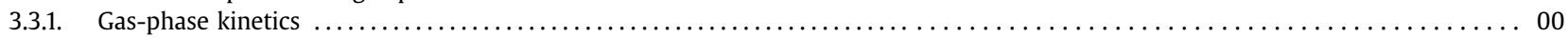

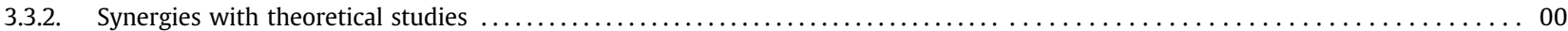

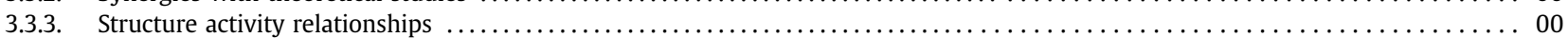

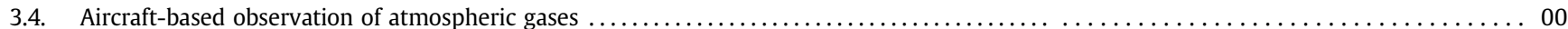

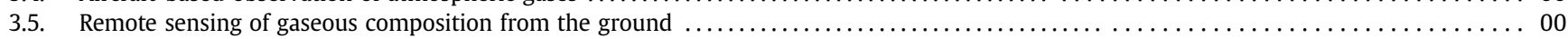

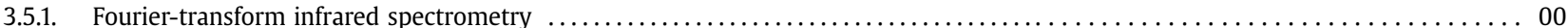

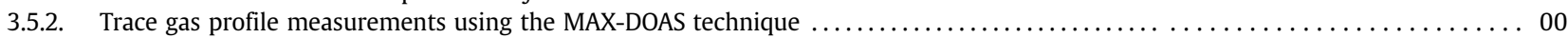

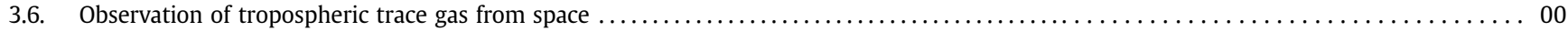

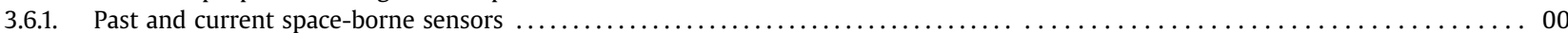

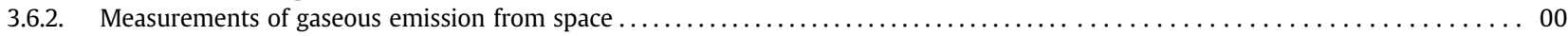

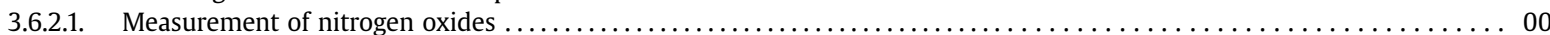

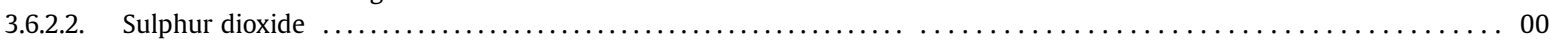

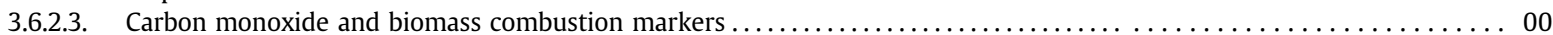

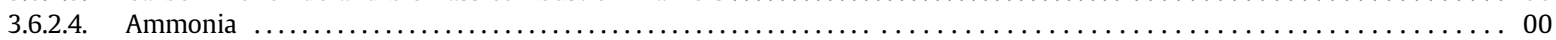

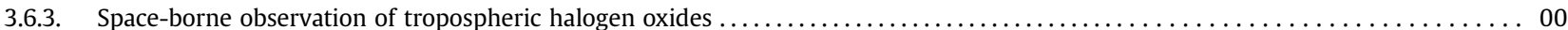

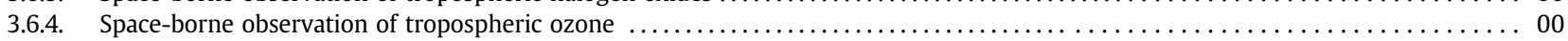

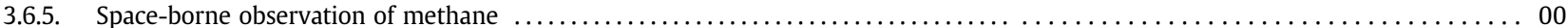

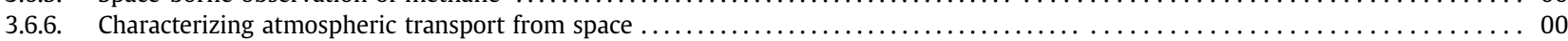

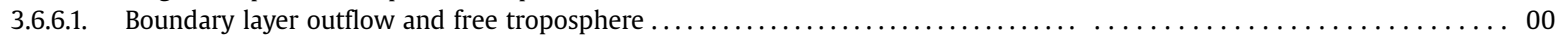

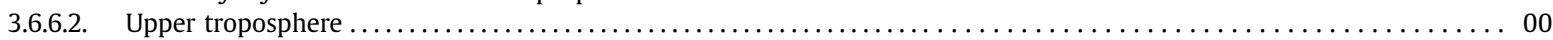

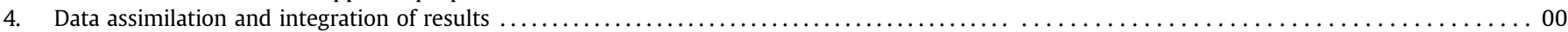

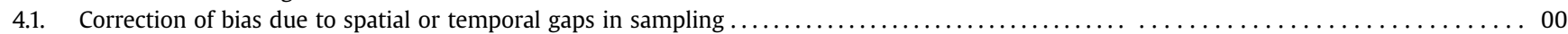

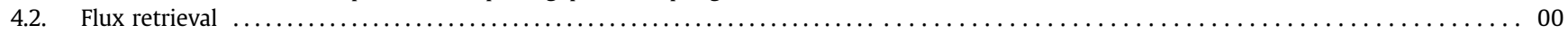

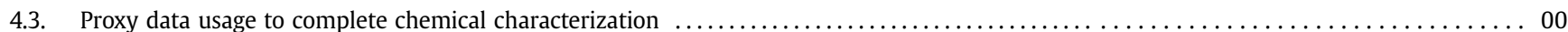

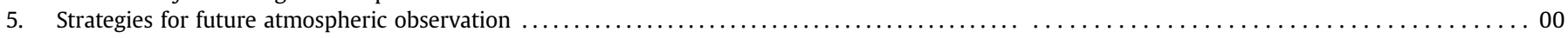

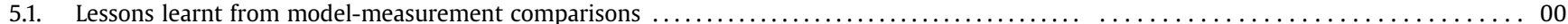

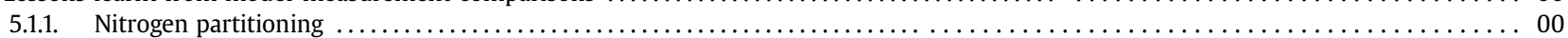

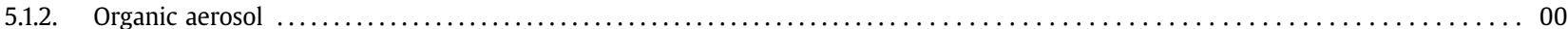

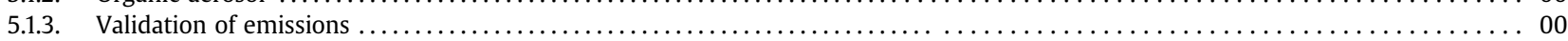

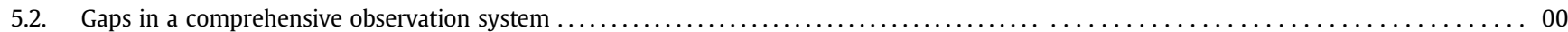

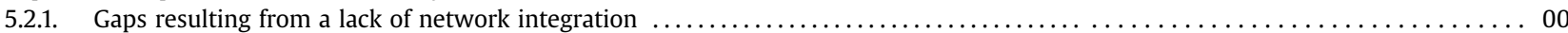

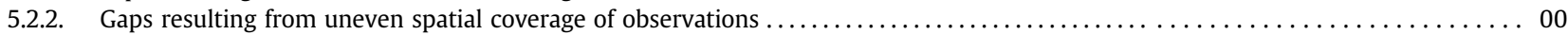

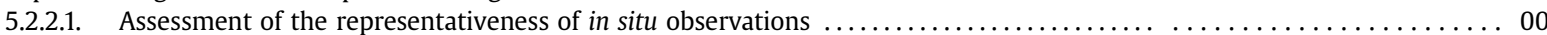

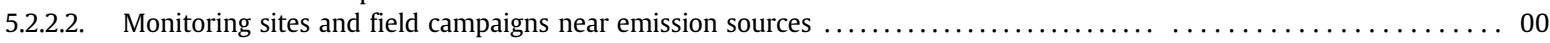

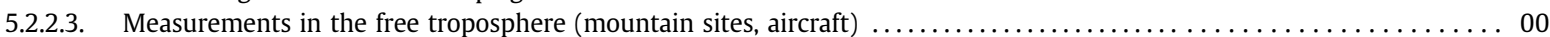

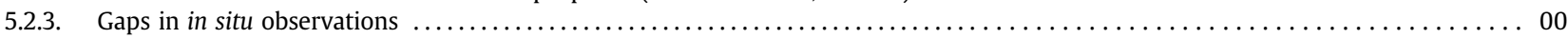

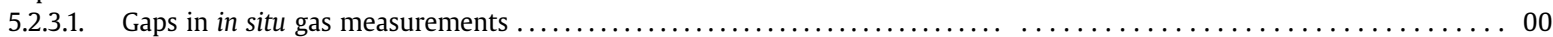

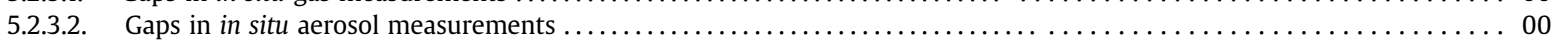

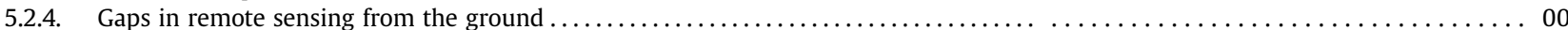

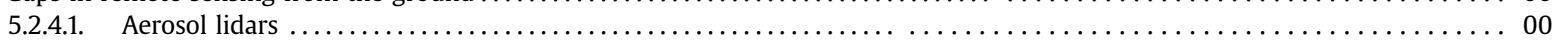

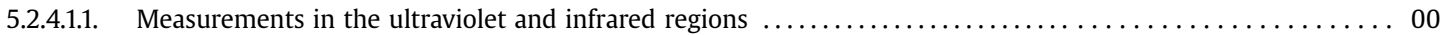

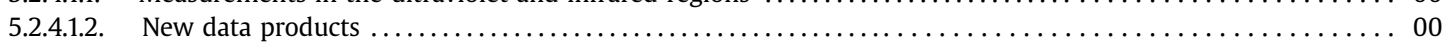

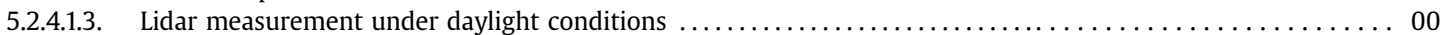

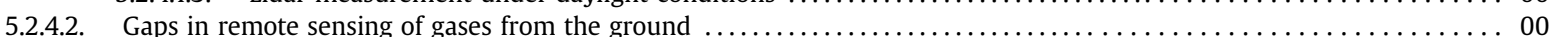

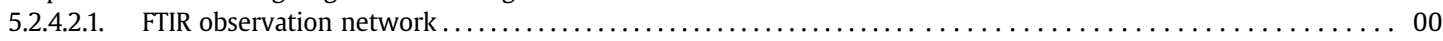

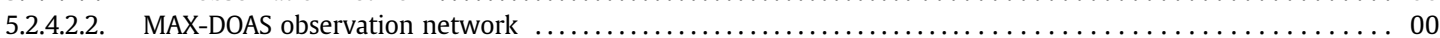




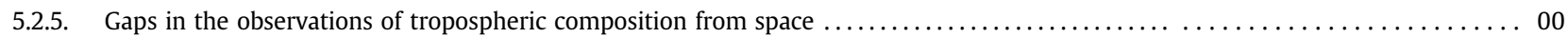

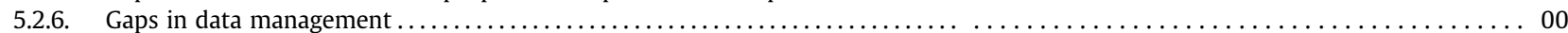

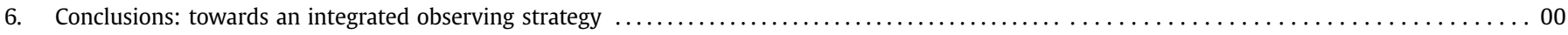

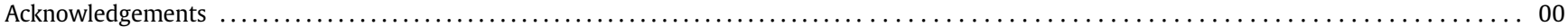

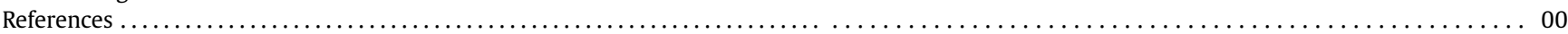

\section{Introduction}

The issue of climate change and improvement of air quality has moved to the top of the global political agenda in many parts of the world. Over the last decade, scientists have made significant progress in understanding the causes and implications of our changing environment. However, many aspects of the global atmospheric system remain uncertain and not fully understood. One of the challenges ahead is linked to the development of a global observation strategy. Emissions of greenhouse and short-lived gases and aerosols due to human activities have altered the atmosphere in ways that strongly affect atmospheric composition and it is expected that emissions will continue to stay at an elevated level in response to fossil fuel burning, tropical deforestation and other industrial activities. Responses to rising temperatures may also alter secondary processes controlling concentrations of key atmospheric species such as tropospheric ozone for which significant increases have recently been monitored in many parts of the world, in particular over mega cities (see Monks et al., in this issue). Similar considerations can be made for the $\mathrm{OH}$ and $\mathrm{HO}_{\mathrm{x}}$ cycle, also controlled by secondary processes (see Monks et al., in this issue). Changes in the emission intensity from both natural and anthropogenic sources, combined with a change in the atmospheric temperature, may furthermore lead to substantial changes in aerosol emissions, and for the generation of secondary organic aerosols, with important and poorly constrained consequences on climate and also on human health in urban areas. Ongoing changes in atmospheric composition may not only affect climate and atmospheric processes but may also lead to measurable impacts on human health, the hydrological cycle and ecosystems, and consequently also on economic developments and the ability of governments to minimize and adapt to the societal and environmental impacts of climate variability and change. There is, therefore, a strong need to understand present and predict future atmospheric composition changes in order to design efficient climate and air quality policies and adapted emission abatement strategies.

Prediction of future changes in atmospheric composition requires information on past and present atmospheric composition. The scientific findings over the last decades have clearly highlighted the need for a more comprehensive approach to studying atmospheric change processes. This includes the use of advanced monitoring techniques, a strong interface with state-of-the-art atmospheric modelling, and the establishment of long-term global and regional atmospheric observations to quantify and understand the current perturbation of the atmospheric cycles of reactive species and aerosols. Contrary to the situation for greenhouse gases and despite the consistent demand for being adopted as scientific priority for decades, dedicated long-term and high quality observations for reactive short-lived species and aerosols have only been initiated recently as part of a response to international conventions and protocols, to GEOS (Global Earth Observation Systems) initiatives and to EU policies in the case of Europe. As a consequence, monitoring has remained limited to a few (often standardized) species and not necessarily to those essential to climate and air quality studies. In fact, observations of atmospheric composition parameters have been an essential activity of atmospheric researchers in developing instrumental tools (advanced analytical techniques) and platforms (instrumented passenger aircraft, ground-based in situ and remote sensing stations, earth observation satellites) that have provided essential information on the state of the atmosphere. However, unlike meteorological parameters that have routinely been collected by meteorological services for decades and for which global satellite observations have existed for 30 years, no integrated system to continuously measure atmospheric composition has been established.

The variability of the atmospheric system and the extreme complexity of atmospheric cycles for short-lived gas and aerosol species have led to the development of complex models to interpret observations, test theoretical understanding of atmospheric chemistry and predict future atmospheric composition. A validation of numerical models requires a comprehensive comparison with multiple types of observations/measurements. This has been a key driving force for the development of new research infrastructures (research aircraft, laboratory facilities, advanced analytical instrumentation and/or monitoring stations) and methodologies for the investigation of atmospheric mechanisms and the generation of original and high quality data for modelling purposes. Data of known quality in terms of precision, accuracy and representativity are of paramount importance for documenting changes in atmospheric composition. The contribution of research infrastructures to the advancement of knowledge in atmospheric science has therefore been essential, in particular over the last decade, with the development of remote-sensing techniques and of new sensors for probing atmospheric composition. Technological advances have permitted accurate measurements of many reactive gases, free radicals and aerosol species and of the complex suite of biogenic and anthropogenic organic compounds and their oxidation products relevant to climate and air quality studies.

Integrated observation strategies have been discussed in a number of studies, in response to a demand from the scientific community and, most of all, from communities related to Global Observing Systems. Important documents have been produced by bringing together providers and users of observations to identify needed products and gaps in observations. Clearly, the objective of integrating satellite, airborne and in situ observation systems has been pursued (and funded) mainly in support of international conventions and protocols (e.g. Vienna Convention on Ozone Depletion, United Nations Framework Convention on Climate Change, Convention on long-range transport of pollutants), the European Commission, and national directives. Delivering regular assessments of the state of the atmosphere in support of European policies and for the benefit of European citizens is, for example, a primary objective of the European GMES (Global Monitoring for Environment and Security) programme. The EMEP (European Monitoring and Evaluation Programme) is a scientifically-based but mostly policy-driven programme under the Convention on Longrange Transboundary Air Pollution for international cooperation to solve transboundary air pollution problems, funded by national governments in Europe, the U.S., Canada, and the European Commission. The IGACO (Integrated Global Atmospheric Chemistry Observation) report on observation strategy is directed towards a globally coordinated development of future observation and 
integration programmes, whose components are either in place or, with careful planning, can be implemented within the next 10 years. The report also emphasizes the need for improving the existing observational systems, thus, it focuses on establishing operational systems with a technically and programmatically feasible long-term solution to meet the identified requirements for atmospheric composition observations. Consequently, previous reports focused primarily on technical issues such as acquisition and processing of space and in situ observations (near-real-time, historic and ancillary), on analysis and forecasting, product generation, on dissemination, archiving, and production of decision-support tools. The plans to build capacity for using atmospheric observation data more effectively into national/international policy frameworks for sustainable development may not always meet scientific challenges. This is why the present paper sets value on measuring atmospheric composition changes which have a stronger scientific focus and a more technology-based approach to the observational requirements identified previously and which are not necessarily driven by policy requirements or by short-term feasibility.

The objective of this paper is, therefore, to assess and discuss technological achievements and challenges ahead not only to construct an integrated atmospheric observing system in support of existing initiatives, but also to answer current scientific issues raised in the context of the companion papers (Fowler et al., in this issue Monks et al., in this issue, Isaksen et al., in this issue). Measuring atmospheric composition changes for climate and air quality applications therefore deals with multiple issues: the detection and validation of trends of tropospheric short-lived species and the relation of these trends to changes in emission intensities; the development of improved methodologies for the detection of key atmospheric compounds in the gaseous and particulate phases and the technological gaps to be overcome; and finally the scientificallybased validation and integration of space-borne, ground based, and airborne observations into a coherent framework of analyses which can be assimilated into modelling studies. These issues are discussed in Sections 2 (aerosol) and 3 (gases), which propose an overview of the most recent and promising techniques related to the detection of atmospheric species that have improved our understanding of the tropospheric cycles in the past several years since the last integration initiative performed within the IGAC (International Global Atmospheric Chemistry) project (Brasseur et al., 2003). Section 4 provides insight into data assimilation techniques, to enhance the significance and scientific output from the observations, and to help to predict short-term changes. Current gaps in our capacity to observe, understand, and predict atmospheric composition changes are discussed in Section 5 which also addresses the challenges ahead to strengthen and optimise an observing system capable of responding to the still open scientific questions. As for the companion papers, the discussion here will be limited to short-lived species (lifetime $<10$ years) in the gaseous and condensed phases addressed within the context of the ACCENT NoE objectives, with a European focus for the observing strategy. It should be noted that all technical aspects related to measurements of emission fluxes or deposition are dealt with by Fowler et al. (in this issue).

\section{Advances in in situ observations of aerosol species}

Tropospheric aerosols are of great importance because of their impact on human health, visibility, continental and maritime ecosystems, and the Earth's climate, requiring dedicated monitoring of their concentrations and properties at the global scale. Aerosol impact on climate is repeatedly mentioned as one of the most uncertain aspects of climate change, and their impact on human health is a growing matter of concern in urban areas. There is a need to design efficient policies for monitoring of aerosols and their precursors and emission abatement strategies in order to understand regional to inter-continental transport of aerosols. The vast majority of current information comes from stations located in urban/peri urban areas and this information is often limited to regulated parameters, such as $\mathrm{PM}_{10}$ or $\mathrm{PM}_{2.5}$ (mass concentration of particles under 10 or $2.5 \mu \mathrm{m}$ in size, respectively), depending on the air quality regulation. This is clearly not sufficient to provide the pertinent information of interest to air quality and climate studies. Variability of advanced aerosol variables, such as aerosol chemical composition possibly size segregated, degree of mixing, optical and hygroscopic properties or 4D distribution (space and time) is now required. Our current knowledge of atmospheric processes relies to a great extent on the substantial technological progress made to characterize chemical compounds and their variability in the atmosphere. In recent years new developments in the techniques and design of laboratory experiments and field measurements related to aerosols have been driven by scientific questions addressing the formation, growth and physicochemical properties of atmospheric aerosol particles (see for instance Jacobson et al., 2000; Kanakidou et al., 2005; Fuzzi et al., 2006). Established techniques and pre-existing instruments have been refined, re-designed and combined in new ways. Instruments such as cloud condensation nuclei (CCN) counters and aerosol mass spectrometers which were previously laboratory prototypes have become commercially available, leading to their widespread use in both laboratory and field site experiments. At the same time, techniques that have been widely used in other scientific fields for many years have been applied successfully in laboratory studies of aerosols - for example, laser desorption ionisation mass spectrometry (LDI-MS) widely used in biology and medicine studies has provided new insight into the chemical composition of secondary organic aerosol particles and chemistry in the particle phase (Kalberer et al., 2004). The development of environmental chambers has also been extremely important, in particular for the understanding of aerosol formation and growth processes. Finally, it is clear that recent knowledge of the variability of aerosol properties at large spatial scale came from passive and active remote-sensing techniques, either from the ground or airborne. As clouds and precipitation are closely linked with aerosols, this overview will also include the microphysics of clouds, the residuals of cloud hydrometeors, and precipitation.

\subsection{Advances in characterizing aerosol chemical composition}

Access to the chemical composition of aerosols has undergone intensive development during the last decade. In recent years, the rapidly-evolving field of aerosol instrumentation has yielded a number of techniques for the on-line analysis of chemical composition that seek to address the problems associated with offline analysis. The main advantage of on-line analytical techniques is their high time resolution compared to off-line analysis methods, and during recent years several new on-line instruments based on mass spectrometry (MS) have been developed or commercialized. In addition, several off-line techniques have been considerably improved by developments of on-line sampling. There is a clear complementarity of both off-line and on-line techniques to provide information needed for air quality and climate studies.

\subsubsection{Off-line characterization of aerosol chemical composition}

The complexity of atmospheric aerosol composition, in particular its organic fraction, led to the development of advanced off-line analysis methods primarily using mass spectrometry including techniques such as laser desorption ionisation MS, LDIMS, (Kalberer et al., 2004), matrix assisted LDI-MS (MALDI-MS) for characterization of oligomers (Surratt et al., 2007) and Fourier transform ion cyclotron resonance MS (Tolocka et al., 2004) for 
assigning unique molecular formula to as many as approximately 450 compounds in the mass range of $200-700 \mathrm{~m} / \mathrm{z}$ (Reinhardt et al., 2007). Chromatographic methods such as gas chromatography (GC) and high performance liquid chromatography (HPLC) separate individual compounds in time in a gas or solvent flow before detection. Aerosol compounds are investigated by methods such as GC-MS (after derivatization typically to enhance volatility) (Yu et al., 1999; Jaoui et al., 2004; Surratt et al., 2006; Szmigielski et al., 2007) and HPLC coupled to other MS techniques such as ion trap MS (Glasius et al., 2000; Müller et al., 2008) and time-of-flight MS (TOF-MS) (Iinuma et al., 2007; Surratt et al., 2008). Such investigations have contributed significantly to our understanding of chemical reactions and aerosol phase products. One example is a group of highly polar compounds, namely the 2-methyltetrols, that were first identified in aerosols from the Amazonian rainforest (Claeys et al., 2004) their discovery led to investigations of their exact route of formation from isoprene (e.g. Böge et al., 2006). Organosulphates have been identified as products of acidcatalyzed heterogeneous reactions involved in the formation of secondary organic aerosol (SOA), providing new information on chemical reactions involved in SOA formation and growth (e.g. Iinuma et al., 2007; Surratt et al., 2007, 2008).

The need for knowledge about thermodynamic properties of organic aerosol components has led to the development of new methods to measure solid and liquid state vapour pressures. One example is thermal desorption mass spectrometry developed and used to measure vapour pressures of low volatility organic aerosol components (Chattopadhyay and Ziemann, 2005; Cappa et al., 2008). Other methods include modified TDMA techniques and single particle optical methods described below.

Off-line methods relying on proton nuclear magnetic resonance $\left({ }^{1} \mathrm{H}\right.$ NMR $)$ spectroscopy have been developed by Decesari et al. (2000) for functional group analysis of the water-soluble fraction of the aerosol OC (WSOC). As shown in Fig. 1 the ${ }^{1} \mathrm{H}$ NMR compositions were used for the source apportionment of organic aerosols (Decesari et al., 2007) and for their representation through molecular models (Fuzzi et al., 2001). ${ }^{1} \mathrm{H}$ NMR spectroscopy is suitable for detecting organic functionalities carrying $\mathrm{C}-\mathrm{H}$ bonds, while ketones or carboxylic groups have to be derivatized before NMR analysis (Moretti et al., 2008). ${ }^{13}$ C NMR spectroscopy could be employed successfully only for heavily loaded aerosol samples (Sannigrahi et al., 2006; Duarte et al., 2007).

\subsubsection{On-line collection coupled with off-line analysis techniques}

Some limitations of off-line techniques (i.e. sampling time integration) have been overcome by the development of on-line collection of aerosol particles followed by fast off-line analysis. The immediate advantages include reduced user interaction, immediacy of data availability and a reduction in the potential for sample contamination or degradation. In addition to addressing these issues, in many cases the new instrumentation expands the scope of the science that is possible.

One generalised technique that has proven to be very powerful is the automation of aerosol sample collection and the application of standard laboratory analytical techniques in situ. An example of such a technique is the on-line Sunset Labs OC/EC analyzer (Bae et al., 2004). This builds on an existing technique whereby a sample on a quartz filter is heated in an inert atmosphere to vapourise or pyrolise any organic species present and the amount of carbon evolved is measured after catalytic conversion (Birch and Cary, 1996). In the second stage of measurement, oxygen is injected to burn off the remaining refractory carbon while the sample is heated further. This enables the measurement of the mass concentration of (operationally defined) organic and elemental carbon.

Speciated information on the organic fraction can also be gained using gas chromatography (GC), as demonstrated by the thermal desorption aerosol GC-MS-FID (TAG) instrument (Williams et al., 2006). The sample is collected on an impactor over a period of typically $30 \mathrm{~min}$ before subjecting it to thermal desorption and GCMS/FID. This instrument is capable of identifying and quantifying various organic species, although in common with all chromatographic techniques, it is limited to the desorbable and elutable fraction. Thermal desorption can also be applied to the inorganic fraction, where commercial instruments have been developed that can measure particulate nitrate and sulphate by measuring the evolved $\mathrm{NO}_{\mathrm{x}}$ and $\mathrm{SO}_{2}$ respectively (Rattigan et al., 2006; Stolzenburg and Hering, 2000).

Impactors and filters are not necessarily the most suitable methods for the collection for certain types of on-line analysis methods, in particular the aqueous techniques. To this end, a number of on-line collectors have become available that activate the sampled particles into the form of water droplets and impact them into a continuously-flowing water stream. This is the basis of the Particle Into Liquid Sampler (PILS) (Orsini et al., 2003; Weber et al., 2001), the Steam Jet Aerosol Collector (SJAC)

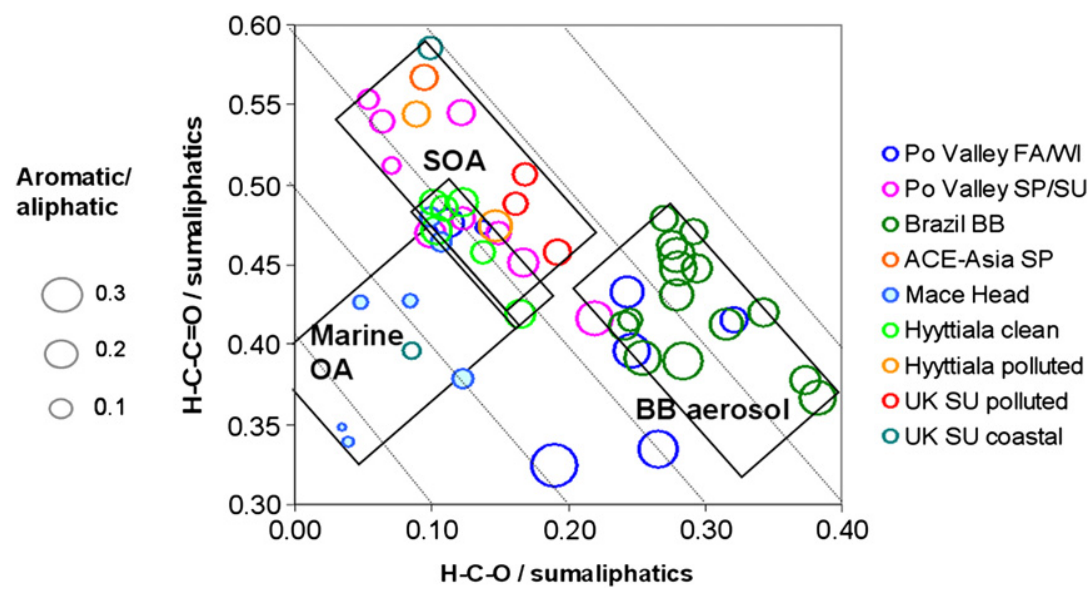

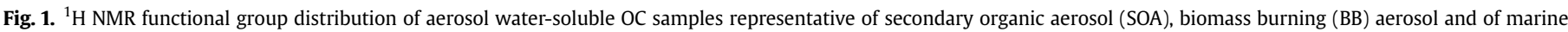

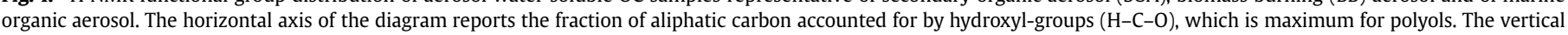

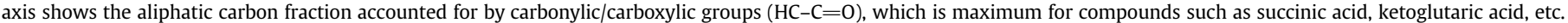

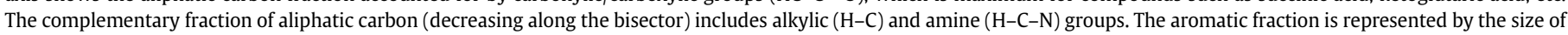
the dots. Adapted from Decesari et al. (2007). 
(Khlystov et al., 1995; ten Brink et al., 2007), and the Wet Effluent Diffusion Denuder-Aerosol Collector (Zellweger et al., 1999). The sample water is periodically removed for analysis (typically around once an hour). Common techniques include ion chromatography which delivers the concentrations of inorganic ions (and certain soluble organic ions), although other methods have been used, such as the analysis of the water-soluble organic carbon fraction (Sullivan et al., 2004), or ion chromatography coupled to MS (Fisseha et al., 2004).

The aforementioned techniques have proved to be very useful, in particular in delivering data sets of increased integrity, time resolution and data coverage. This increases the scope of the interpretative methods that can be applied to the data (e.g. factor analysis Williams et al., 2007). However, as these are still bulk samples, they suffer from the same limitations as the off-line analysis methods in that the time resolution is still limited to typically tens of minutes. Information regarding size is limited to what can be gleaned using impactors or cyclones in the inlet systems, and no information regarding mixing state is retained at all.

\subsubsection{On-line characterization of aerosol chemical composition}

Around the mid-90s, the field of on-line aerosol mass spectrometry began to emerge as a method for on-line composition analysis of atmospheric aerosols (Coe and Allan, 2006; Hinz and Spengler, 2007; Suess and Prather, 1999). Applications of aerosol mass spectrometry in aerosol studies have increased considerably during recent years, and the technique has provided new information on aerosol formation, composition and reactions with high time resolution (e.g. Alfarra et al., 2006; Gross et al., 2006; Hartz et al., 2007; Weitkamp et al., 2007). The instruments are either capable of analysing single particles or an ensemble of particles, and an overview of available techniques is presented in recent reviews (Murphy, 2007; Nash et al., 2006). The instruments can then be divided further into two classes based on the desorption method; laser desorption and thermal desorption. While similar in concept, these two techniques result in fundamentally different data products which require different approaches to data analysis and interpretation. Table 1 summarizes the different aerosol mass spectrometry techniques discussed in this paper.

2.1.3.1. Single particle mass spectrometry. The general method is to introduce particles into a vacuum chamber, desorb them, ionise the constituents and analyse them using various forms of mass spectrometry. Laser desorption and ionisation (LDI) (Murphy, 2007) is the technology employed in the TSI Aerosol Time Of Flight Mass Spectrometer (ATOFMS) (Gard et al., 1997) and in in-house designs such as the Particle Analysis by Laser Mass Spectrometry (PALMS) (Murphy and Thomson, 1995) and Single Particle Analysis and Sizing System (SPASS) (Erdmann et al., 2005) or the Single Particle Laser Ablation Time-of-flight mass spectrometer (SPLAT) (Zelenyuk and Imre, 2005). A few aerosol on-line analyzers need to be connected to an instrument selecting a certain particle size (DMA) to provide size distribution and size chemical composition. In this group, we refer by example to the Thermal Desorption Particle Beam Mass Spectrometer (TDPBMS) (Tobias et al., 2000) or the Thermal Desorption Chemical ionisation Mass Spectrometer (TDCIMS) (Voisin et al., 2003).

Aerosol particles are drawn into a vacuum chamber using a nozzle or aerodynamic lens to focus the particles into a beam. Skimmers and differential pumping remove the majority of the gas phase material. The particles are detected using light scattered from low-powered optical lasers, which trigger an appropriately timed pulse from a more powerful laser (typically in the ultraviolet range). This pulse transfers enough energy to the particle to both desorb and ionise the constituents, producing large numbers of ions from individual particles. Time-of-flight mass spectrometry is then used to analyse these ions, gated to the initial laser pulse. Because both negative and positive ions are generated, two mass spectrometers of opposing polarity are frequently used to deliver two mass spectra per particle. Highly accurate aerodynamic sizing information is also provided by inspecting the delay between the detection lasers (Salt et al., 1996).

The use of high-powered laser pulses means that the instruments are particularly well suited to the study of refractory particles, with elemental carbon, minerals, and metals being particularly well detected. However, the real strength of these instruments is the ability to probe mixing states directly, something that other online instruments can only do in an indirect or limited manner. They are also very well suited for the real-time identification of specific types of particles, providing that they create a unique enough response in the instrument. This property makes the technique particularly promising for real-time bio-aerosol detection and identification (Adams et al., 2008; Fergenson et al., 2004).

The main limitation of the LDI instruments is that during desorption, the chemical components interact and the energy and charge are not distributed evenly among the molecular species

Table 1

Summary of the measurement techniques discussed in this section.

\begin{tabular}{|c|c|c|c|c|c|}
\hline Technique & $\begin{array}{l}\text { Time resolution } \\
\text { (typical) }\end{array}$ & Sizing & Inorganic detection & Organic detection & Mixing state information \\
\hline $\begin{array}{l}\text { On-line OC/EC } \\
\quad \text { (Sunset Labs) }\end{array}$ & $1 \mathrm{~h}$ & None & $\begin{array}{l}\text { Quantification of } \\
\text { elemental carbon }\end{array}$ & Quantification of total organic carbon & None \\
\hline On-line GC (TAG) & $1 \mathrm{~h}$ & None & None & $\begin{array}{l}\text { Identification of categories and some specific } \\
\text { species within the elutable fraction. } \\
\text { Quantification of variations and some } \\
\text { absolute concentrations }\end{array}$ & None \\
\hline Thermal reduction (SPA) & $10 \mathrm{~s}$ & None & $\begin{array}{l}\text { Quantification of sulphate } \\
\text { or nitrate, depending } \\
\text { on gas analyzer used }\end{array}$ & None & None \\
\hline $\begin{array}{l}\text { Aqueous collection } \\
\text { (PILS, SJAC) }\end{array}$ & $\begin{array}{l}1 \mathrm{~h}(5 \mathrm{~min} \\
\text { on aircraft) }\end{array}$ & None & $\begin{array}{l}\text { Quantification of soluble } \\
\text { ions (if used with IC) }\end{array}$ & $\begin{array}{l}\text { Quantification of simple acids } \\
\text { (if used with IC). Quantification of } \\
\text { water-soluble organic carbon } \\
\text { (if used with TOC analyzer) }\end{array}$ & None \\
\hline $\begin{array}{l}\text { Single Particle Mass } \\
\text { Spectrometry } \\
\text { (ATOFMS, PALMS, SPASS) }\end{array}$ & Real-time & $\begin{array}{l}\text { Aerodynamic } \\
\text { (individual } \\
\text { particles) }\end{array}$ & $\begin{array}{l}\text { Qualitative detection } \\
\text { of many species } \\
\text { (matrix dependent) }\end{array}$ & $\begin{array}{l}\text { Qualitative detection of some } \\
\text { categories (matrix dependent) }\end{array}$ & $\begin{array}{l}\text { Grouping of particles } \\
\text { according to composition } \\
\text { using cluster analysis }\end{array}$ \\
\hline Aerodyne AMS & $\begin{array}{l}1 \mathrm{~min}(10 \mathrm{~s} \text { on } \\
\text { aircraft, } 10 \mathrm{~Hz} \\
\text { in flux mode })\end{array}$ & $\begin{array}{l}\text { Aerodynamic } \\
\text { (ensemble) }\end{array}$ & $\begin{array}{l}\text { Quantification of } \\
\text { non-refractory species } \\
\text { (e.g. ammonium, } \\
\text { nitrate, sulphate) }\end{array}$ & $\begin{array}{l}\text { Quantification of organic matter. Some } \\
\text { quantitative information on functionality }\end{array}$ & $\begin{array}{l}\text { Can glean limited } \\
\text { information from } \\
\text { size-resolved data }\end{array}$ \\
\hline
\end{tabular}


(known as matrix effects). Also, most molecules will undergo some degree of fragmentation, dependent on the amount of energy available. The extent to which the charge and energy distribution occurs depends on the exact overall composition, meaning that the relative intensity of the ions detected is not necessarily representative of the composition of the parent particle. This is further confounded by the fact that the amount of laser energy absorbed is dependent on the particle size and optical absorption profile. The result is that these instruments are generally not capable of delivering quantitative data on particle composition. These effects can be mitigated by using two laser pulses to separate the desorption and ionisation stages (Salt et al., 1998).

Another challenge is that particle detection is limited to how well the particle beam intersects with the focal point of the desorption laser. As a result, these instruments can have very strong detection biases based on particle size (Allen et al., 2000), depending on the inlet design and instrument geometry (Huffman et al., 2005). The inlet itself should be matched to the type of particles under investigation, with nozzles being suited to coarse particles and aerodynamic lenses (Liu et al., 1995a,b) being optimal for the submicron fraction.

Data analysis presents a major challenge, as data on hundreds of thousands of particles are typically collected during a single field campaign and much effort has been made in developing clustering techniques that reduce the data into time-resolved number concentrations of classes of particles. This type of data product is particularly useful in source apportionment studies (Beddows et al., 2004; Guazzotti et al., 2003). There are many methods in use (such as hierarchical clustering Murphy et al., 2003 and ART-2a Phares et al., 2001), the relative merits of which are subject of debate (Rebotier and Prather, 2007). In particular, due to the evaporation and ionisation source by laser technique, quantification is very difficult to obtain. Their key qualities are their accuracy, computational cost and suitability for real-time analysis.

2.1.3.2. The aerosol mass spectrometer (AMS). The aerosol mass spectrometer (AMS) collects an ensemble of particles and thermally evaporates chemical compounds at $200-900{ }^{\circ} \mathrm{C}$, followed by electron impact (EI) ionisation and detection with either quadrupole MS (Jayne et al., 2000) or time-of-flight MS (DeCarlo et al., 2006; Drewnick et al., 2005). EI is a hard ionisation method, and fragments of organic compounds rather than their molecular ions are detected. This new generation of instruments allows size distribution and information about the chemical composition of the particles to be measured with a high time resolution at the same time. Different categories of instrument have been developed and have recently been reviewed by (Sullivan and Prather, 2005).

Rather than delivering mass spectra of individual particles, the AMS gives mass spectra of the observable particle ensemble. During data analysis, specific chemical species can be extracted from the mass spectra based on marker peaks and through the application of calibration data, quantitative mass concentrations can be delivered (Allan et al., 2003, 2004). Aerodynamic sizing information is provided by a chopper wheel that periodically modulates the particle beam (Jimenez et al., 2003). Additional sizing data can also be provided by using a light scattering module (Cross et al., 2007).

The main limitation of the instrument is that it is only capable of detecting the non-refractory components, so while ammonium sulphate, nitrate and organic matter are detected very well, the instrument is blind to elemental carbon, sea salt and dust. Additionally, the quantitative capabilities of the instrument are limited to the focusing range of the aerodynamic lens used on the inlet, which is typically around $50-700 \mathrm{~nm}$ (Zhang et al., 2002, 2004). Another problem is that in general, around $50 \%$ of solid-phase particles 'bounce off' the vapouriser without being detected. This can be corrected for through the comparison with an accompanying measurement (Drewnick et al., 2003), but the phenomenon has been shown to be reproducible and objective parameterisations now exist to remove the artefact (Crosier et al., 2007a; Matthew et al., 2008).

The principal advantage of the instrument over off-line methods is the vast increase in the resolution of the data products, allowing for a time resolution of around tens of seconds and a sizing resolution of tens of bins per decade (detection limits permitting). A faster data collection and delivery mode enables flux measurements by eddy covariance (Nemitz et al., 2008) and improves signal quality when performing airborne measurements (Crosier et al., 2007b).

An important development of the instrument has been the replacement of the quadrupole mass spectrometer with a time-offlight mass spectrometer (Tofwerk model C-TOF) (Drewnick et al., 2005); using orthogonal extraction, this has increased sensitivity by around two orders of magnitude, of particular use for sampling in remote environments and on aircraft (DeCarlo et al., 2008; Sorooshian et al., 2008). A larger, high-resolution variant (the Tofwerk HR-TOF) (DeCarlo et al., 2006) is capable of resolution greater than 4000 , permitting elemental analysis of the peaks and aiding in the separation of components. As there is a significant trade-off with ion sensitivity, the instrument typically alternates between high and low resolution modes during sampling.

$70 \mathrm{eV}$ Electron ionisation carries an inherent disadvantage in causing extensive fragmentation; in the AMS, there will also be fragmentation through thermal decomposition of the parent ions on the surface of the vapouriser. Unlike LDI, if the vapouriser temperature and electron energy are kept constant, fragmentation is reproducible, but nonetheless information regarding the parent species is lost, especially when sampling complex mixtures, as is usually the case with atmospheric organic aerosols. Alternative ionisation methods have been tried, such as vacuum ultraviolet (VUV) ionisation (Northway et al., 2007) using 10-eV photons to ionise the organic vapours with less fragmentation but at the cost of overall sensitivity, particularly to inorganics. Also, thermal denuding upstream of the AMS can separate components by volatility (Huffman et al., 2008).

However, even with these techniques, the AMS is mainly limited to reporting general organic functionality rather than specific species when analysing ambient data. By inspecting certain peaks known to be associated with types of functional groups (e.g. $m /$ $z=44$, indicative of di- and polycarboxylic acids), qualitative statements on the degree of oxidation of the organics can be made (Alfarra et al., 2004; Allan et al., 2004). If high-resolution data are available, more quantitative estimates of elemental ratios of carbon, hydrogen, oxygen, nitrogen and sulphur can be made (Aiken et al., 2007, 2008).

Because of the additive nature of AMS data and the fact that organic aerosols can originate from a number of distinct sources and processes, it is often possible to apportion the reported organics using numerical analysis. An early method is the so-called HOA/OOA analysis (Zhang et al., 2005), whereby the time-resolved ensemble organic mass spectrum is separated into two factors based on markers in the mass spectrum, producing a profile mass spectrum and quantitative time series for hydrocarbon-like and oxygenated organic aerosol (HOA and OOA). This approach is fairly robust in apportioning organics to primary and secondary sources in polluted continental sites (Zhang et al., 2007). More recently, positive matrix factorization (PMF) (Paatero, 1997) has been used to separate the data into any number of quantitative factors without the need for specifying markers in the mass spectrum. This has enabled a quantitative apportionment and profiling of additional factors in various environments, such as biomass burning and cooking (Lanz et al., 2007). Additionally, it can also split the OOA factor into a number of sub-factors, which allows insights to be 
gained into the variations in composition of the secondary organic aerosol fraction (Lanz et al., 2007).

\subsection{Use of isotope chemistry for aerosol studies}

Among other off-line analytical techniques used for the characterization of atmospheric aerosols, isotopic analyses of aerosol samples, mainly collected on filters, is a rapidly growing field of research. The following discussion is split into radioactive and stable isotopes.

\subsubsection{Radioactive isotopes}

Many natural radioactive isotopes are used for atmospheric process studies. As an example, tracers such as radon and its daughters track the dynamical changes of the lower atmosphere and can be used as 'clocks' for atmospheric processes. Lugauer et al. (2000) used radon daughters to study vertical transport processes in the Alps. Hammer et al. (2007) applied lead-210 observations as a diagnostic tool for assessing the spatiotemporal variability of related chemical aerosol species.

The use of carbon-14 analysis to distinguish between fossil and non-fossil sources, for example particulate matter from wood burning and traffic (Szidat et al., 2007, 2009), has been favoured by progress in mass spectrometry, requiring much less material than before. This intrinsic isotopic information characterizes the sources of ambient PM independent of its history regarding emission conditions or atmospheric transport. Such ${ }^{14} \mathrm{C}$ measurements have been performed for quite some time, but only a discrimination of OC and EC prior to the ${ }^{14} \mathrm{C}$ determination reveals the full power of this method: EC is often dominated by fossil fuel, while OC typically has a higher biogenic fraction. These features are not accessible in a ${ }^{14} \mathrm{C}$ analysis of the total carbon (Szidat et al., 2006). This technique is especially powerful in combination with positive matrix factorization of e.g. aerosol mass spectrometric data (Lanz et al., 2008). Gelencsér et al. (2007) also used measured radiocarbon concentrations to constrain their source apportionment of carbonaceous aerosol.

\subsubsection{Stable isotopes}

Stable isotope measurements complement concentration measurements and also provide useful hints concerning the origin of airborne particulate matter, because individual sources often induce different isotopic signatures. Recent developments involve measurements of the isotopic ratios of light elements such as carbon, nitrogen, oxygen and sulphur in secondary aerosol species such as carbonaceous aerosol species, nitrate, ammonium and sulphate. In this case, isotopic ratios reveal not only the nature of the aerosol source but also the atmospheric processing of their precursors, thereby supplying singular information on the budget of atmospheric reactive trace species. Applications of these techniques for improving the knowledge of atmospheric chemical composition and reactivity are reviewed in Monks et al. (in this issue).

\subsubsection{Carbonaceous species}

Methods for fraction- or compound-specific isotopic analyses of carbonaceous aerosol species are becoming increasingly available (Fisseha et al., 2009). The carbon isotopic ratio (expressed in terms of $\delta^{13} \mathrm{C}$, see Monks et al., in this issue) can be measured on the elemental and organic fraction of carbonaceous aerosol materials by coupling a stepwise thermal desorption/combustion OC/EC analyzer (e.g. Sunset instrument, see Section 2.1.2) with an IRMS operated in continuous-flow mode (Huang et al., 2006). Among organic species, the classical discrimination based on water-solubility properties (e.g. WSOC versus WinSOC fractions Pio et al., 2007) can be applied to isotopic analyses performed using TC/EA (or analogous) devices or chemical conversion of organic matter to
$\mathrm{CO}_{2}$, yielding $\delta^{13} \mathrm{C}$ measurements of these two bulk fractions of organic aerosols. Ion chromatography (Fisseha et al., 2006) or chemical derivatization prior to GC (Kawamura and Watanabe, 2004) can be used to separate components of water-soluble organic matter (especially organic acids) prior to isotopic analysis. Techniques have also recently been developed to measure the ${ }^{13} \mathrm{C}$ isotopic composition of water-insoluble components of organic aerosols, such as fatty acids (e.g. Matsumoto et al., 2001; Fang et al., 2002) and alkanes (e.g. Bendle et al., 2006), using a continuousflow GC separation system followed by a combustion interface prior to injection into the IRMS.

\subsubsection{Nitrate}

Atmospheric nitrate is produced upon oxidation of nitrogen oxides, so that the isotopic composition of nitrate provides strong constraints on their budget (e.g. Morin et al., 2008; Monks et al., in this issue). Two analytical approaches are currently in use to measure the isotopic composition of nitrate. "Classical" techniques for the measurements of $\delta^{15} \mathrm{~N}$ and $\delta^{18} \mathrm{O}$ of nitrate involve the combustion of nitrate salts (Revesz et al., 1997; Kornexl et al., 1999), and require nitrate concentration and purification steps prior to the combustion to avoid interferences with other species such as organics and sulphate (Silva et al., 2000; Savarino et al., 2001; Chang et al., 2004; Michalski et al., 2008). Combustion techniques have been extended to the measurement of the isotope anomaly ( or ${ }^{17} \mathrm{O}$ excess, see Monks et al., in this issue) $\left(\delta^{17} \mathrm{O}=\delta^{17} \mathrm{O}-0.52 \times \delta^{18} \mathrm{O}\right.$ or any equivalent definition) of nitrate, combusted in the form of $\mathrm{AgNO}_{3}$ (Michalski et al., 2002). Minimal amounts needed for such analyses are in the order of $5-10 \mu \mathrm{mol} \mathrm{NO}_{3}^{-}$, which is problematic for many atmospheric applications. Following the pioneering approach of Sigman et al. (2001), techniques based on the conversion of nitrate to $\mathrm{N}_{2} \mathrm{O}$, followed by on-line isotopic analysis of $\mathrm{N}_{2} \mathrm{O}$ (e.g. Komatsu et al., 2008), have been developed for measuring the isotopic composition of smaller amounts of nitrate. The conversion of nitrate to $\mathrm{N}_{2} \mathrm{O}$ can either be done chemically (Mcllvin and Altabet, 2005) or using denitrifying bacteria lacking the $\mathrm{N}_{2} \mathrm{O}$-reductase (Sigman et al., 2001; Casciotti et al., 2002; Kaiser et al., 2007). Measuring the comprehensive isotopic composition of nitrate (including $\delta^{17} \mathrm{O}$ ) requires either using two types of bacteria inducing a different incorporation of oxygen atoms from nitrate into $\mathrm{N}_{2} \mathrm{O}$ (Coplen et al., 2004) or performing an additional $\mathrm{N}_{2} \mathrm{O}$ decomposition step into $\mathrm{N}_{2}$ and $\mathrm{O}_{2}$, on a gold catalyst at $800-900{ }^{\circ} \mathrm{C}$ (Cliff and Thiemens, 1994 ; Kaiser et al., 2007; Komatsu et al., 2008). Analytical performances of such systems typically lie within less than $0.5 \%$ for $\delta^{15} \mathrm{~N}, \delta^{18} \mathrm{O}$ and $\delta^{17} \mathrm{O}$ using a few tens of nmol nitrate (Casciotti et al., 2002; Kaiser et al., 2007), allowing atmospheric studies to be performed at daily to subdaily temporal resolutions, if needed.

\subsubsection{Ammonium}

Zhang et al. (2007) report on a new sensitive technique for the analysis of $\delta^{15} \mathrm{~N}$ of ammonium samples, which could be easily implemented for atmospheric applications. The technique involves the conversion of ammonium to $\mathrm{NO}_{2}^{-}$by $\mathrm{BrO}^{-}$ions, followed by chemical conversion of $\mathrm{NO}_{2}^{-}$to $\mathrm{N}_{2} \mathrm{O}$ and subsequent isotopic analysis (Mcllvin and Altabet, 2005). Precisions better that $0.3 \%$ on a minimum amount of $10 \mathrm{nmol} \mathrm{NH}_{4}^{+}$are achieved using this method, making it more suitable for atmospheric applications than previous ones such as the "diffusion" method (Yeatman et al., 2001).

\subsubsection{Sulphate}

Quite similarly to nitrate, $\mathrm{S}$ and $\mathrm{O}$ isotopic ratios of sulphate provide insights into its sources and into the atmospheric fate of its precursors $\left(\mathrm{SO}_{2}\right)$ (e.g. McCabe et al., 2006). S and O both possess more than two stable isotopes $\left({ }^{16} \mathrm{O},{ }^{17} \mathrm{O}\right.$ and ${ }^{18} \mathrm{O}$ for oxygen, ${ }^{32} \mathrm{~S},{ }^{33} \mathrm{~S},{ }^{34} \mathrm{~S}$ and ${ }^{36} \mathrm{~S}$ for sulphur) allowing the study of non-mass dependent isotopic 
fractionation induced by some atmospheric oxidation ( $O$ isotopes) or photolysis ( $\mathrm{S}$ isotopes) reactions (see Monks et al., in this issue). We only present here methods developed and used for the determination of more than one isotopic ratio of a given element in sulphate. Following separation from other major anions $\left(\mathrm{Cl}^{-}, \mathrm{NO}_{3}^{-}\right)$by ion chromatography (Savarino et al., 2001) or chemical techniques (Bao, 2006) sulphate samples are usually precipitated as barium or silver sulphate. Several techniques are then used to determine its isotopic composition, including for triple oxygen isotopic ratios $\left(\delta^{17} \mathrm{O}\right.$ and $\delta^{18} \mathrm{O}$ ) $\mathrm{BaSO}_{4} \mathrm{CO}_{2}$-laser fluorination systems (Bao and Thiemens, 2000) or $\mathrm{Ag}_{2} \mathrm{SO}_{4}$ pyrolysis (Savarino et al., 2001). Isotopic ratios of sulphur from sulphate are obtained by converting barium sulphate $\left(\mathrm{BaSO}_{4}\right)$ to silver sulphide $\mathrm{Ag}_{2} \mathrm{~S}$ (through several chemical steps), which is subsequently fluorinated by $\mathrm{BrF}_{5}$ in a sealed nickelized tube (full details are given in Savarino et al. (2001), and references therein). The produced $\mathrm{SF}_{6}$ is purified and analyzed mass-spectrometrically. Baublys et al. (2004) and Poulson (2005) present new methods to measure the ${ }^{33} \mathrm{~S} /{ }^{32} \mathrm{~S}$ along with the generally only measured ${ }^{34} \mathrm{~S} /{ }^{32} \mathrm{~S}$. Both techniques rely on the conversion of the sulphate to $\mathrm{SO}_{2}$ gas, free of the oxygen isobaric interference. Precisions of a few tenths of a \% can be achieved using samples in the $\mu \mathrm{mol}$ size range, enough to study major atmospheric processes but still inadequate to study dynamical processes at subdaily timescales.

The recent use of nanoscale secondary ion mass spectrometry (NanoSIMS) for isotopic measurements of individual sulphate particles (Winterholler et al., 2008; Sinha et al., 2008) holds promise, even if only $\delta^{34} \mathrm{~S}$, and not the comprehensive $\mathrm{S}$ isotopic composition of individual particles was presented and discussed in this pioneering study. Along the same lines, Sanusi et al. (2006) recently presented a method to measure the $\delta^{34} \mathrm{~S}$ composition of methanesulphonic acid (MSA), an important intermediate species associated with the atmospheric sulphur cycle.

\subsection{Physical, thermal and hygroscopic properties of atmospheric aerosols}

Physical aerosol properties such as the number concentration or the size distribution are important for a number of reasons. In urban and rural air, these ultrafine particles generally comprise the major fraction of the particle number concentration (e.g. Bukowiecki et al., 2002). The size of the particles determines in which part of the inhalation system they are deposited. The size of the particles also determines to a great extent their optical properties and their rates of deposition to the surface (Fowler et al., in this issue). Thermal and hygroscopic properties give insight into chemical properties of the aerosol, and are among the very few methods (except single particle analysis methods) that allow for an assessment of the degree of internal versus external mixture. As an example, the fraction of particles freshly emitted by traffic versus the fraction of aged particles can be determined (Baltensperger et al., 2002).

\subsubsection{Particle number concentration and size distribution}

2.3.1.1. Particle number concentration. Condensation particle counters (CPCs) were developed to measure the integral particle number concentration of sub-micrometre aerosol particles larger than a certain threshold diameter. Because of the convenience of their operation, CPCs represent the most frequently used measurement instrument in atmospheric aerosol research. Since about 2000, progress in CPC technology has taken place mainly in two fields. First, working fluids other than to the most commonly used (butanol) were shown to be applicable, for instance water (Hering and Stolzenburg, 2005; Hering et al., 2005) or perfluorinated organic compounds like FC-43 (Brock et al., 2000; Gallar et al., 2006). While the latter proved to be advantageous over butanol at low operation pressures (Hermann et al., 2005), a clear statement for the applicability of water-CPCs cannot be made. Some studies state that water-CPCs are well suited for ambient aerosols, i.e. for field studies (e.g. Biswas et al., 2005), while other studies especially use the difference in the particle activation caused by the different water-solubility of the particles in order to investigate particle chemical composition (Kulmala et al., 2007a).

A second development in the last decade is the rediscovery of the mixing-type-CPCs (nowadays almost exclusively the conductive-cooling-CPCs are used). The new mixing-CPCs are capable of measuring particle concentrations very fast (Wang et al., 2002) and it seems possible to reach lower threshold diameters compared to the conductive-cooling-CPCs (e.g. Gamero-Castaño and De la Mora, 2000; Kim et al., 2003; Sgro and De la Mora, 2004). Particle measurements with a high time resolution (in the range of tenths of a second or even less) are desirable when investigating rapidly changing particle emissions like automobile exhausts, or when using a fast moving measurement platform like aircraft. A lower threshold diameter would help to understand better the process of particle nucleation. Unfortunately, at present (2009) no commercial version of these new mixing-type CPCs is available in Europe.

In laminar flow CPCs, the final droplet size after condensation decreases with decreasing particle size for particles $<10 \mathrm{~nm}$. This size-dependent droplet growth can be utilized to derive the size distribution of the smallest detectable particles. The method evaluates the amplitudes of the electronic pulses caused by single particles in the optical detector of the CPC. From the pulse height spectra, size distributions can be obtained by a mathematical inversion (Weber et al., 1998). Since all particles larger than $10 \mathrm{~nm}$ grow to the same final size, this pulse height analysis (PHA) technique is limited in its range to particles $<10 \mathrm{~nm}$. Sipilä et al. (2008) introduced an operation mode where the supersaturation inside a laminar flow CPC is increased to the extent that homogeneous nucleation occurs. They illustrated how the effect of homogeneous nucleation could be separated from the pulse height spectra of ambient nanoparticles leading to the detection of particles smaller than $2 \mathrm{~nm}$.

Several CPCs having different lower cut-off sizes can be combined to measure particle concentrations across different size ranges. By subtracting the readings corresponding to different size ranges, a relatively coarse-gridded size distribution can be constructed for the size range below the uppermost CPC cut-off size. Two or more CPCs operating with different working liquids can be combined into a CPC battery. The CPC battery makes use of different vapour pressures of condensable vapours over particle surfaces of different chemical composition. Kulmala et al. (2007a) presented a CPC battery composed of four individual CPCs, representing a $2 \times 2$ matrix of different cut-off diameters ( 3 and $11 \mathrm{~nm}$ ), as well as different working liquids (n-butanol and water). The principle of the CPC battery (see Fig. 2) is that lipophilic (or hydrophobic) particles of a certain diameter just below the CPC detection limit will neither be activated by the butanol nor the water-based CPC. A hygroscopic particle of the same dry size, by contrast, will eventually be activated by the water-based CPC as a result of the increased affinity between the particle and the vapour, but not by the butanol CPC. Significant numbers of hygroscopic particles near the size of the detection limit will therefore show as a difference between the readings of the butanol and water-based CPCs. The CPC battery is suitable for the examination of the activation properties of particles $<20 \mathrm{~nm}$ and, therefore, their physicochemical properties.

2.3.1.2. Particle size distribution. A complete size distribution of atmospheric aerosol requires more than a single instrument. For the submicron aerosol, scanning and differential mobility particle sizers (SMPS and DMPS, respectively) have been used for more than 20 years and, despite slight design improvements, no noticeable 


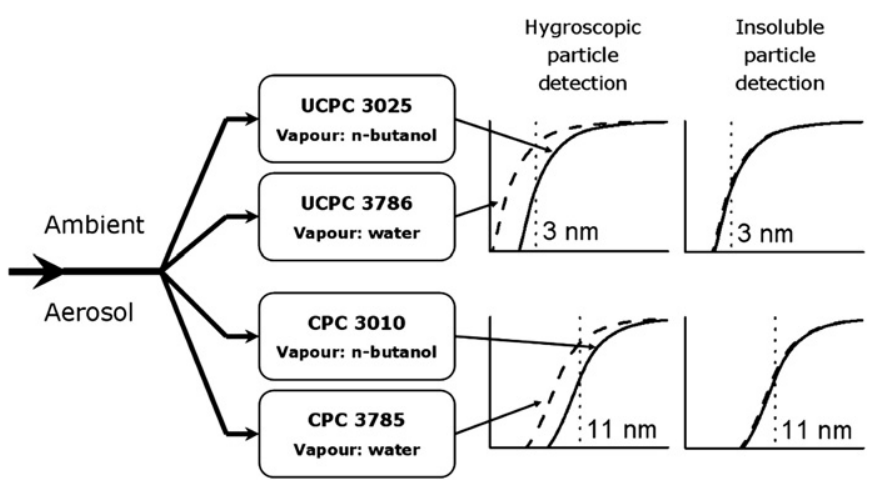

Fig. 2. Operating principle of the condensation particle counter battery ( $\mathrm{CPCB})$. From Kulmala et al. (2007a).

improvement needs to be mentioned in this review. However, an important feature during the last decade is the fact that the number of commercially available and custom-made SMPS and DMPS, and therefore the number of users, has increased significantly. A few instrument inter-comparison workshops recently performed showed significant deviations between different types of mobility size spectrometers, which leads to questions about the validity of most retrieved size distributions. The reasons for these differences lie in the setup of the mobility size spectrometers as well as in different inversion routines and correction schemes (Helsper et al., submitted for publication). Furthermore, the quality of these measurements remains unknown if system parameters such as flow rates, temperature and relative humidity are not recorded. This fact is of particular importance if the mobility size spectrometers are used in long-term measurements.

A disadvantage of both the SMPS and DMPS is their relatively low time resolution (typically a few minutes). New instruments such as the fast mobility particle sizer (FMPS) or engine exhaust particle sizer (EEPS) have recently been developed to overcome this disadvantage (Johanson et al., 2004). The FMPS measures the particle mobility diameter in the range $5.6-560 \mathrm{~nm}$ with a time resolution of $1 \mathrm{~s}$ using a flow rate of $10 \mathrm{l} \mathrm{min}^{-1}$. The instrumental technique is based on particle electrical mobility analysis. A unipolar charger produces a defined number of charges on the particles which enter the instrument through a PM1 (particulate matter of particles with an aerodynamic diameter $d_{\mathrm{p}}<1 \mu \mathrm{m}$ ) cut-off. The particles that flow through the region of different electrical fields are repelled by the voltage from the central column. When the particles hit the outer cylinder, consisting of multiple cylindrical rings, the particles create a current which is measured by electrometers. This instrument has also been used to obtain size distributions on board mobile platforms (Weimer et al., 2009).

A major advance in recent years is the development of techniques for nanoparticle and ion measurements (Kulmala et al., 2007b). The neutral cluster and air ion spectrometer (NAIS) is a relatively novel instrument which measures mobility distributions of charged as well as neutral aerosol particles and molecular clusters. The instrument is manufactured by Ariel Ltd (Tartu, Estonia), and measures across an electrical mobility range between 0.0010 and $2.39 \mathrm{~cm}^{2} \mathrm{~V}^{-1} \mathrm{~s}^{-1}$, corresponding to a diameter range of approximately $0.8-47 \mathrm{~nm}$. The NAIS is based on the air ion spectrometer (AIS) but additionally uses unipolar charging of sampled particles by corona discharge to increase their detectability (Mirme et al., 2007). Electrostatic postfilters are installed to remove the excess of ions generated by the corona discharge. Thereafter, particles are classified in an electrical mobility analyzer, and finally detected as an electric current with a set of parallel electrometers. To keep statistical fluctuations from the electrical signals low, a time resolution of $5 \mathrm{~min}$ is currently recommended. The lower detection size of uncharged ambient particles in the NAIS is estimated to be around $2 \mathrm{~nm}$, because the signal of smaller particles tends to interfere with that of the internally generated discharge ions. The NAIS instrument is currently undergoing detailed characterization experiments, but has also been deployed at several atmospheric research stations worldwide to investigate the dynamics of new particles from gaseous precursors. It is a very interesting complement to the more common SMPS/DMPS measurement as it provides unambiguous evidence for nucleation of new particles, as shown by Venzac et al. (2008), and Fig. 3.

2.3.1.3. Physical measurements for determination of the density of aerosol particles. The density is one of the crucial properties needed to properly characterize aerosol particles and it is one of the parameters needed to link electric mobility and aerodynamic diameter and number and mass distributions (McMurry et al., 2002). It is therefore essential in order to describe aerosol dynamics. McMurry et al. (2002) proposed the use of a combination of DMA and Ehara's particle mass analyzer to measure atmospheric particle density. Kannosto et al. (2008) measured the temporal evolution of particle density for the nucleation, Aitken and accumulation modes using a low pressure impactor in parallel with a scanning mobility particle sizer (SMPS). The density behaviour in the nucleation mode suggests that different compounds are involved in the nucleation and growth of new particles. Katrib et al. (2005) studied the density changes following the ozonolysis of oleic acid coatings on latex spheres, while Bahreini (2005) estimated the effective density of SOA from ozonolysis of several hydrocarbons. Studies on the effective density also improve our understanding of exhaust emissions (Skillas et al., 1998). In soot particles emitted from diesel engines, the effective density decreases for increasing particle size due to agglomeration as the size of soot particles increases (Park, 2003). Thus, it has been suggested that the PM mass emission could be retrieved from size distribution measurements as an alternative to filter collection and off-line analysis (Maricq and Xu, 2004). Particle density has also been the subject of theoretical investigations; DeCarlo (2004) pointed out that the non-unique definition of effective density found in the literature can lead to incorrect inter-comparison of different experimental results.

\subsubsection{Measurement of hygroscopic and thermal properties and flow tubes}

An important method for measuring hygroscopic and thermal properties is the tandem differential mobility analyzer (TDMA) technique which has been used and re-designed for a variety of purposes. TDMAs provide indirect information on particle composition and size in almost real time and, hence, offer a powerful advantage over many other chemistry-based methods that require longer time integration.

2.3.2.1. TDMA systems for aerosol hygroscopic properties. The hygroscopicity TDMA (H-TDMA) system is widely used for determination of particle water uptake and to validate functional groupbased model predictions like UNIFAC (Moore, 2008). The principle of the H-TDMA system has been used for more than twenty years (Liu et al., 1978) to measure size-dependent hygroscopic properties of sub-micrometre particles with high temporal resolution (Liu et al., 1978). A comprehensive review of the H-TDMA principle and applications is given by Swietlicki et al. (2008). The basic principle of the H-TDMA technique is to monitor the growth of a particle of known diameter in a high $\mathrm{RH}$ environment (typically around $90 \%$ $\mathrm{RH}$ ). The H-TDMA is generally composed of two differential mobility analyzers (DMA-1 and DMA-2) used in series, a particle counter, and various pumps and humidity control devices etc. A polydisperse 

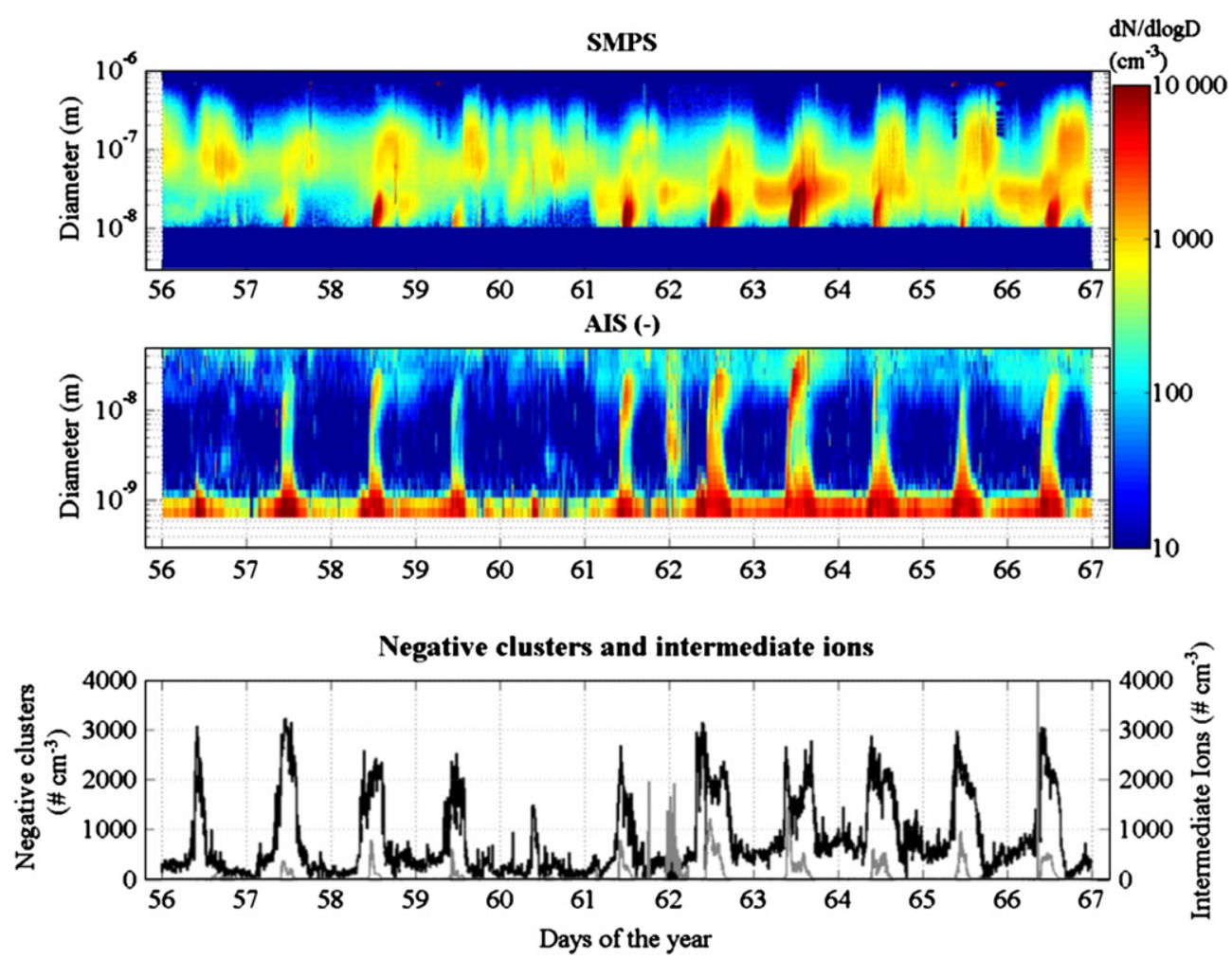

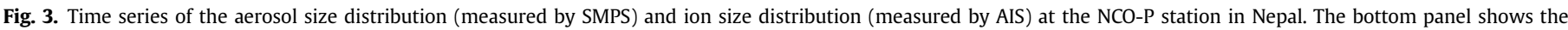

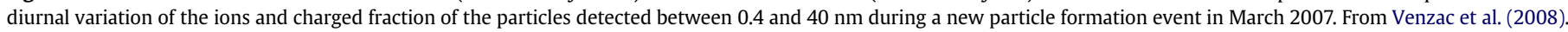

aerosol distribution at ambient relative humidity enters the system, and is dried as it enters DMA- 1 ( $\mathrm{RH}<10 \%)$. DMA- 1 is used to select a monodispersed distribution of a given size. The aerosol exiting DMA-1 is humidified and then enters DMA-2. DMA-2 coupled with the particle counter is used to measure the size distribution of the humidified monodispersed aerosol, hence providing a growth factor spectrum. In addition, for a given particle size, differential growth of the particles is used to characterize the mixing state of the aerosol population. Internal mixing is characterized by homogeneous growth while an externally mixed particle population is evidenced by the presence of multiple hygroscopic modes. Up to now, H-TDMA systems have not been commercially available, and all existing systems are based on customised designs which have been improved according to control quality criteria to sort and evaluate data, and in the data evaluation algorithms. Duplissy et al. (2009) summarized technical information and proposed a more standardized application of H-TDMA techniques aiming for high data quality and data comparability. Gysel et al. (2009) proposed a standardization of H-TDMA inversion schemes leading to H-TDMA systems that now can be used for long-term monitoring operation. The organic TDMA is a new development based on the same operating principle as the H-TDMA but with organic liquids (such as ethanol) as the condensing vapour instead of water (Joutsensaari et al., 2004). This setup has the potential to provide insight into the presence and properties of organic molecules in atmospheric aerosols including newly-formed ultrafine particles (Joutsensaari et al., 2004).

2.3.2.2. TDMA systems for aerosol thermal properties. Another type of tandem DMA technique is the so-called volatility TDMA where the aerosol particles are heated between the two DMAs. It has been designed and used to monitor the non-volatile fraction of chamber generated aerosol particles (Paulsen et al., 2006). The volatility technique provides a suitable tool to measure on-line physicochemical properties of aerosol particles with high size resolution by heating the aerosol (typically up to $300{ }^{\circ} \mathrm{C}$ ). Results permit speculation about chemical composition without exact details. Non-volatile fractions of sub-micrometre aerosols are assumed to consist of soot or long-chain hydrocarbons, as well as sea salt and mineral dust in the upper accumulation mode range. The technique itself has been well known for many years but has been significantly improved and frequently used during the last 10 years. The volatility TDMA (V-TDMA) measures the mixing state of the non-volatile fraction of a few pre-selected particle sizes. A thermodenuder (TD) coupled to a single size spectrometer provides the non-volatile fraction of the whole aerosol population, but gives no information on the mixing state. Several versions of a TD have been built during the past decade (e.g. Burtscher et al., 2001; Wehner et al., 2002, but also commercial ones are available from TSI and Dekati) with the main goal to quantify fractions of soot-like compounds with a high time and size resolution. Other applications of the volatility technique have recently greatly increased within the last years and cover a wide range: chamber studies to detect changes in volatility (e.g. Kalberer et al., 2004), vehicle exhaust studies to distinguish between primary emitted soot and secondarily produced aerosol material (e.g. Sakurai et al., 2003), and the combination with an AMS (Huffman et al., 2008) to improve the knowledge about the chemical composition of the non-volatile fraction at different temperatures. H-TDMA and VTDMA techniques (VH-TDMA) have also been coupled by several groups to investigate the hygroscopic properties of the non-volatile fraction of ambient sub-micrometre aerosols and the influence of the more volatile fraction of the particle on hygroscopic growth (Jonhson et al., 2004; Villani et al., 2007, 2008; Sellegri et al., 2008). The principle of a VH-TDMA is shown in Fig. 4.

The modified TDMA setup for measurement of evapouration rates of dried aerosol particles (Rader and McMurry, 1986) has further been developed for measurements of evapouration rates of aqueous solution droplets and determination of subcooled liquid as 

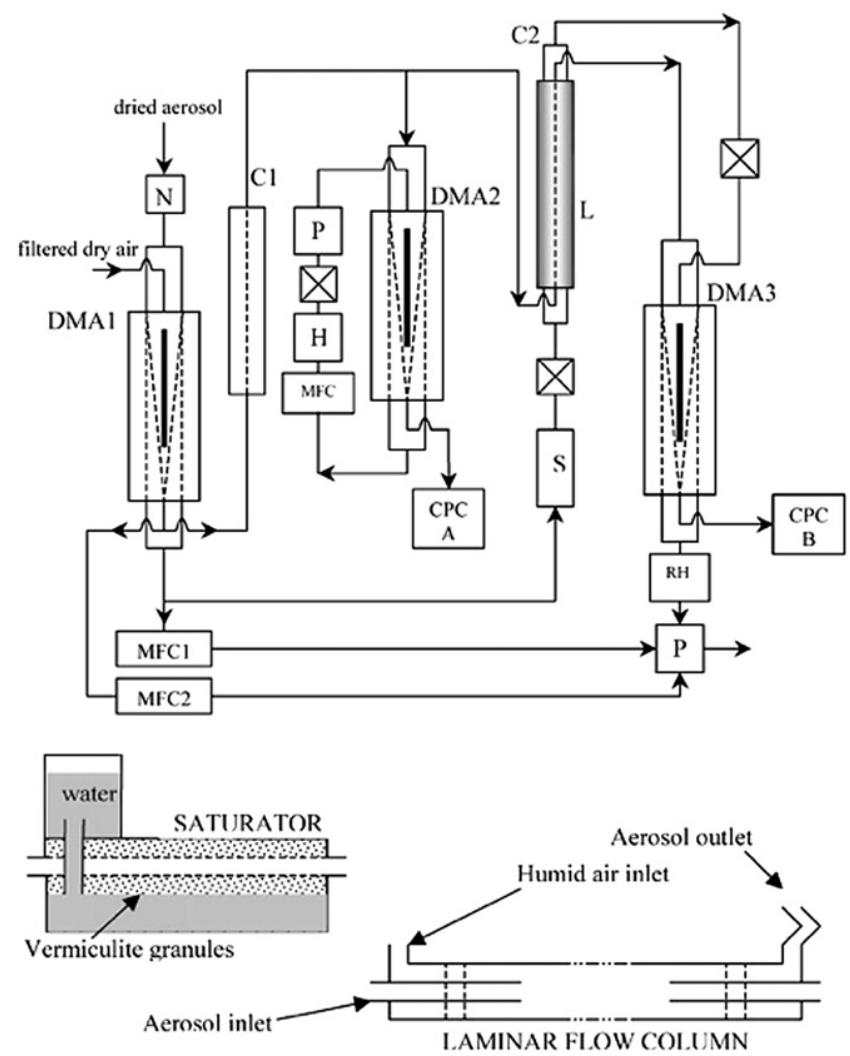

$\begin{array}{llll}\text { DMA1, 2,3 } & \text { Electrostatic Classifiers } & \text { H } & \text { Heat exchanger } \\ \text { N } & \text { Neutralizer } & \text { S } & \text { Saturator } \\ \text { C1 } & \text { Conditioner-volatilization } & \text { P } & \text { Pump } \\ \text { C2 } & \text { Conditioner-humidification } & \text { CPC } & \text { Condensation particle counter } \\ \text { L } & \text { Laminar flow column } & \text { B } & \text { Valve } \\ \text { Q } & \text { HEPA Filter } & \text { MFC } & \text { Mass flow controller } \\ \text { RH } & \text { Relative humidity sensor } & & \end{array}$

Fig. 4. Description of VH-TDMA system. Adapted from Johnson et al. (2004).

well as solid state vapour pressures of secondary organic aerosol components (Riipinen et al., 2006; Riipinen et al. 2007; Koponen et al., 2007).

\subsection{Environmental chamber studies}

Over the last seven years several new environmental aerosol chamber facilities have been constructed (e.g. Paulsen et al., 2005; Cocker et al., 2001; Stanier et al., 2007; Hartz et al., 2005; Carter et al., 2005) where mixtures of reactive gases and/or aerosol particles are generated and studied under controlled conditions of temperature, pressure, relative humidity, and electromagnetic radiation, and a suite of instruments has been used to investigate aerosol properties. An important advance was to minimize loss of gas molecules and particles to the walls by constructing large chambers (volume $>10 \mathrm{~m}^{3}$ ) which increases the ratio of volume to surface area in the chambers. As an alternative, smaller chambers can be operated in a dynamic mode with equal inlet and outlet flows (e.g. VanReken et al., 2006; Kang et al., 2007). The majority of environmental chambers are operated at tropospheric conditions (in terms of temperature and pressure). New insight has been gained into the importance of temperature, relative humidity, and light source and intensity (Warren et al., 2008) on aerosol yield and properties. The measurement of potential aerosol mass (PAM) based on rapid oxidation of precursor gases with extreme amounts of oxidants and UV light in a small flow-through chamber is a further new development (King et al., 2007) which remains to be tested and compared, for example, with results from large-scale environmental chambers and the atmosphere.

In most chamber studies aerosol particles are generated by nucleation and growth of gas phase oxidation products or by condensation of low volatility species on seed particles. Recent studies have also used direct emissions from plants as aerosol precursors in environmental chambers (McFiggans et al., 2004; Joutsensaari et al., 2005; VanReken et al., 2006). The variety of instruments connected to environmental chambers has considerably increased over the last decade: Cloud condensation nuclei (CCN) counters to study the activation properties of freshly generated organic particles (Hartz et al., 2005; VanReken et al., 2005; Prenni et al., 2007; Duplissy et al., 2008) and hygroscopic/volatility tandem differential mobility analyzers (H/V-TDMA) to measure aerosol hygroscopicity (Baltensperger et al., 2005; Varutbangkul et al., 2006; Duplissy et al., 2008) and volatility (Paulsen et al., 2006) are two examples. The chemical composition of aerosol particles generated in environmental chambers is often analyzed using a suite of different techniques as discussed in Section 2. The Leipzig Aerosol Cloud Interaction Simulator (LACIS, see Fig. 5) is a new development in flow tubes with a great potential to investigate particle/droplet hygroscopic growth under sub-saturated conditions, particle activation and subsequent dynamic growth under supersaturated conditions within one setup (Stratmann et al., 2004). It allows for studies of particle phase transitions over a wide range of temperatures and relative humidities including supersaturations (Wex et al., 2005). LACIS can be operated under both subsaturated and supersaturated conditions. Relative humidities up to 99.5\% (sub-saturated) and supersaturations down to $0.1 \%$ can be adjusted reproducibly. LACIS has been used to investigate the hygroscopic growth and activation behaviour of different types of aerosol particle, successfully connecting high relative humidity hygroscopic growth and activation behaviour for selected inorganic and organic substances (Wex et al., 2007 and reference therein). LACIS complements the AIDA facility used for investigating heterogeneous ice nucleation of relevant aerosols at simulated tropospheric cloud conditions (e.g. Field et al., 2006; Möhler et al., 2005, 2008a,b).

\subsection{Aerosol/cloud in situ measurement technologies}

Clouds can have a considerable effect on the Earth's climate but processes regarding their formation, persistence, and dissipation

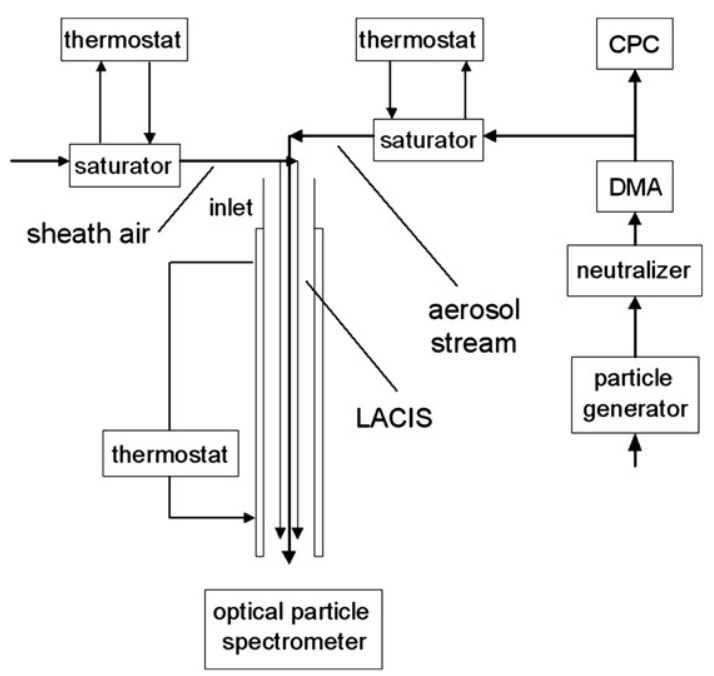

Fig. 5. Schematic of the Leipzig Aerosol Cloud Interaction Simulator (LACIS). 
are still very uncertain. The interactions between water vapour and aerosol particles in the atmosphere underlie many of the physical processes that govern cloud formation and optical properties. In particular, processes of aerosol cloud interactions above and below freezing temperature are key climate-relevant phenomena that depend on the fundamental physical properties of water vapour, liquid water, and ice. The need for more information on aerosol/ cloud interaction has led to new developments in CCN (cloud condensation nuclei) and IN (ice nuclei) characterization, especially regarding the quantification of water uptake by organic and soot particles of various origins.

\subsubsection{Cloud condensation nuclei}

Cloud condensation nuclei counters are widely used instruments for investigating particle activation to cloud droplets above $100 \% \mathrm{RH}$, and are now commercially available through DMT (Droplet Measurement Technologies) (Lance et al., 2006; Roberts and Nenes, 2005). In this instrument, a wettable column of a length of $0.5 \mathrm{~m}$ is heated to increasingly higher temperatures along the flow direction of the aerosol. The flow is directed from top to bottom, and the aerosol is confined to a narrow beam along the centre line, surrounded by sheath air. The instrument uses the fact that water vapour diffuses faster than heat to establish a level of supersaturation along the centre line, which is thought to be constant over the whole length of the column. Below the column, light scattered from the particles/droplets is measured for particles larger than $500 \mathrm{~nm}$. From the scattering signal, a droplet size distribution in the range from 0.75 to $10 \mu \mathrm{m}$ is determined. Particles that have not been activated to droplets are too small to be detected. The activated fraction of particles as a function of adjusted supersaturation or initial particle size is determined from the number of droplets counted divided by the number of particles supplied to the instrument. This instrument has been used in a variety of laboratory (e.g. Asa-Awuku et al., 2009; VanReken et al., 2005) and field applications (e.g. Dusek et al., 2006). An alternative to the use of CCN chambers is the use of Counter-flow virtual impactors (CVIs) coupled with interstitial aerosol samplers in clouds. The technique has been applied successfully in a number of studies such as Sellegri et al. (2003), Marinoni et al. (2004), Laj et al. (2001), Mertes et al. (2001, 2005), Verheggen et al. (2007a,b).

\subsubsection{Ice nucleation}

In mixed-phase clouds at temperatures between $0{ }^{\circ} \mathrm{C}$ and about $-38{ }^{\circ} \mathrm{C}$, heterogeneous ice nucleation is important in initiating a series of processes strongly influencing cloud evolution and eventually leading to precipitation. Heterogeneous ice nucleation in mixed-phase clouds is induced by insoluble or solid aerosol particles, the so-called ice nuclei (IN). It is known from previous laboratory and field studies that both inorganic particles like mineral dust (Roberts and Hallett, 1968; Schaller and Fukuta, 1979) and organic particles like bacteria or pollen can act as ice nuclei in deposition nucleation and freezing modes at various temperatures and supersaturations with respect to ice. Black carbon was reported to be enriched in ice crystal residuals (Cozic et al., 2008). Nevertheless, there is a lack of information about the abundance and distribution of tropospheric IN and in particular about their relation to properties of the atmospheric aerosol. In recent years, an increasing number of laboratory experiments have addressed the efficiency of relevant aerosol particles to induce ice nucleation in tropospheric clouds, and several new techniques and instruments have been built to measure the IN concentration continuously both in laboratory and field applications.

Laboratory studies of ice nucleation made use of existing and newly developed or improved experimental techniques like continuous flow diffusion chambers (Chen et al., 2000; Salam et al.,
2006), aerosol flow tubes (Wise et al., 2004), flow diffusion cells with optical microscopy (Dymarska et al., 2006), cold stage with optical microscopy (Zuberi et al., 2006), cold stage cells with optical or Raman microscopy (Kanji and Abbatt, 2006; Knopf and Koop, 2006), differential scanning calorimetry (Marcolli et al., 2007), detection of phase transition by X-ray diffraction (Murray and Bertram, 2008) and cloud simulation chambers (Möhler et al., 2005). Important new results have been e.g. obtained on the ice nucleation properties of ammonium sulphate aerosols (Abbatt et al., 2006; Zuberi et al., 2002), mineral dust particles (Durant and Shaw, 2005; Field et al., 2006; Kanji and Abbatt, 2006; Knopf and Koop, 2006; Marcolli et al., 2007; Salam et al., 2006), soot particles (Dymarska et al., 2006), organic particles (Wise et al., 2004), bacterial cells (Möhler et al., 2008a), and on the effect of inorganic and organic coating layers on the ice nucleation properties of mineral dust (Archuleta et al., 2005; Möhler et al., 2008b) and soot particles (Möhler et al., 2005). More recent work with potentially important implications for atmospheric cloud formation addressed the effect of the suppression of ice nucleation in highly viscous, glassy-state organic particles (Murray, 2008; Murray and Bertram, 2008; Zobrist et al., 2008).

Recently several groups have improved existing or have built new instruments for IN measurements. New instruments (Jones et al., 2008; Cotton, 2008; Saito and Murakami, 2008) have been built following the design of the continuous flow diffusion chamber (CFDC) which was developed by Rogers (1988) and already used during many field and laboratory projects (e.g. Archuleta et al., 2005; DeMott, 2002; Rogers et al., 2001). Stetzer et al. (2008) developed a new continuous flow diffusion instrument called the Zurich Ice Nucleation Chamber (ZINC) for laboratory use and a portable version (PINC), both with a new design of vertically oriented parallel plates. A new instrument called the Frankfurt Ice Nucleation Chamber (FINCH) was developed which makes use of rapid mixing for continuous IN measurements (Bundke et al., 2008). Finally, static diffusion chambers called FRIDGE for IN measurements on filter samples (Ardon et al., 2008; Klein et al., 2008) have been recently developed. The AIDA (Aerosol Interaction and Dynamics in the Atmosphere) facility hosted the Fourth International Ice Nucleation Workshop (ICIS-2007) for contrasting and comparing up-to-date methods and instruments for measuring ice nucleation processes and ice nuclei concentrations (Möhler et al., 2008c). Experiments in the large-scale environmental chamber AIDA have elucidated ice nucleation characteristics of atmospheric dust samples (Field et al., 2006). Based on several types of laboratory results Abbatt et al. (2006) suggested that a cirrus cloud formation pathway involving solid ammonium sulphate particles as ice nuclei exists. A promising new facility that recently came into operation is the dynamic cloud chamber of the Meteorological Research Institute (MRI) in Tsukuba, Japan (Tajiri et al., 2008), which was built following the design of the Colorado State University cloud chamber (DeMott and Rogers, 1990).

Recent laboratory experiments have tested the relationship between heterogeneous ice freezing temperatures and water activity of the solution for different solutes and ice nuclei (IN). Zuberi et al. (2002) observed the nucleation of ice in $\left(\mathrm{NH}_{4}\right)_{2} \mathrm{SO}_{4}-$ $\mathrm{H}_{2} \mathrm{O}$ particles with mineral dust immersions using an optical microscope, while Archuleta et al. (2005) focused on mineral dust particles with sulphuric acid coatings in a continuous flow ice thermal diffusion chamber.

Recently, considerable effort has been spent in studying the role of organics as IN; dicarboxylic acid nucleation was explored using a differential scanning calorimeter (Zobrist et al., 2006) and the indirect aerosol effect of oxalic acid was modelled and discussed; inorganic salt aqueous solution droplets with long chain alcohol films were studied in a droplet freezer setup (Cantrell and Robinson, 2006); several combinations of organic/silicate solutes/IN systems were investigated by Zobrist et al. (2008). These works 
suggest that the nucleation theory based on water activity may be applied also to heterogeneous freezing, besides that of homogeneous nucleation as previously stated by Koop et al. (2000).

\subsubsection{Microphysics of clouds}

There is a variety of instruments for the microphysical characterization of clouds, and in some areas substantial progress has been made. Until recently, characterization of droplets in many in situ studies was performed using a particulate volume monitor (PVM-100, Gerber Scientific) measuring the cloud liquid water content (LWC) using the light scattering of a laser beam, a forward scattering spectrometer probe (FSSP-100) for the size distribution of cloud hydrometeors from 2 to $47 \mu \mathrm{m}$, or $2 \mathrm{D}-\mathrm{C}$ probe for the measurements of ice crystal and droplets dimension in the range of 25-250 $\mu \mathrm{mAs}$ these instruments were developed some time ago they are not further described here. Instead, a new generation of instruments for droplet/ice crystal characterization has been developed in recent years.

o The Airborne Droplet Analyser (ADA), manufactured by TSI Inc. is a new development to measure the size distribution of hydrometeors and is based on phase Doppler anemometry. It utilises an argon ion laser to generate two cross-directed laser beams with a small sample volume in the interfering region. The interference fringes are modified by the presence of cloud droplets and the size and velocity of the droplet can be determined. The technique can discriminate between spherical droplets and aspherical particles and so is capable of rejecting the small ice crystals in the cloud. In addition, changes in refractive index can be measured and used to reject non-liquid water particles. The ADA has a much larger size range than the FSSP and is typically operated with approximately 200 channels ranging from 1 to $200 \mu \mathrm{m}$.

- The hydrometeor size spectrum can be measured by a cloud particle imager (CPI, SPEC Inc. 230X). The CPI records images of individual cloud particles (from 5 to $2300 \mu \mathrm{m}$ in diameter) passing through its sample area. Since standard processing of CPI data yields only qualitative size distributions, a novel analysis procedure was employed to obtain quantitative information (Connolly et al., 2007, and references therein). The CPI was originally designed for aircraft use but can also be used for ground-based operation by fitting it with a high volume aspirator. A very innovative use of CPI has been performed by Lawson et al. (2006) incorporating a polar nephelometer (PN) that measures the scattering phase function with one CPI. Correlated measurements between the two instruments permitted the linking of diffusion of light by ice particles to crystal size and shape under the conditions of the South Pole (see Fig. 6).

- The Counter-flow Virtual Impactor (CVI) samples and evapourates hydrometeors above a certain size inside clouds, releasing dry residual particles. Downstream of the CVI the condensed water is completely evapourated in a particle free, dry carrier gas, followed by the analysis of the dry residual aerosol particles. Based on the design of these CVIs, Mertes et al. (2007) developed an Ice-CVI that allows for the separation of small ice particles from large ice crystals, cloud droplets and interstitial aerosol particles. The first stage of the Ice-CVI consists of a virtual impactor which removes hydrometeors larger than $20 \mu \mathrm{m}$ in diameter. Then, an inertial preimpactor removes the supercooled droplets as they freeze upon impaction on plates held below $-5{ }^{\circ} \mathrm{C}$, while small ice crystals bounce off and are carried with the sample air stream. Finally, aerosol particles that bounce and have diameters below $5 \mu \mathrm{m}$ are removed by a Counterflow Virtual Impactor,

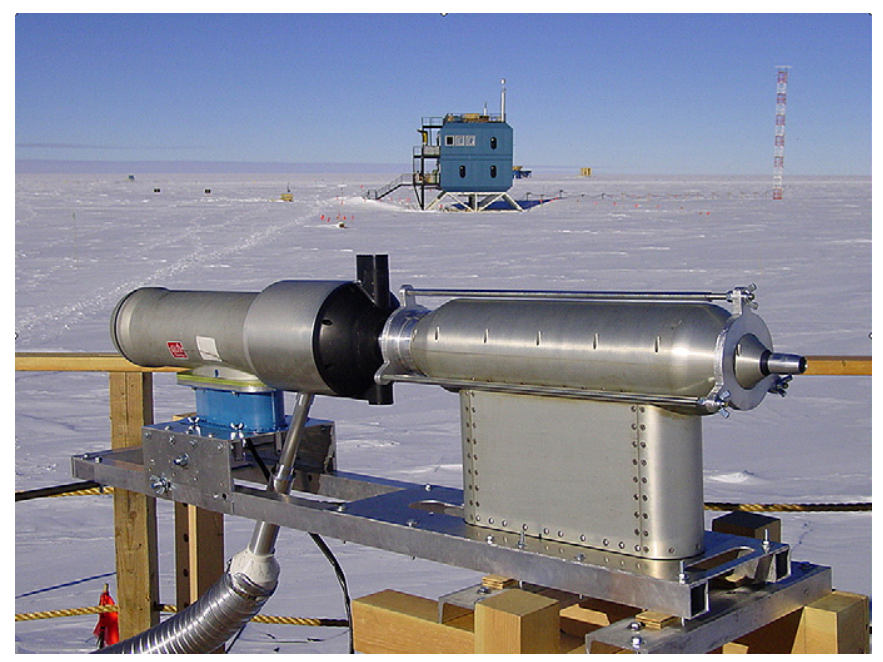

Fig. 6. Measurement of crystal shape by cloud particle imager coupled with measurement of diffusion phase function by polar nephelometer (Lawson et al., 2006). Image courtesy of J.-F. Gayet (CNRS).

whereas the larger ice crystals (diameters between 5 and $20 \mu \mathrm{m}$ ) overcome the counterflow. Analogous to the regular $\mathrm{CVI}$, the water of the ice crystals is then sublimed and the residual particles are then characterized. Progress in aerosol metrology, in particular in aerosol mass spectrometry now allows an on-line characterization of cloud droplet residuals behind CVIs (Drewnick et al., 2007).

\subsubsection{Cloud chemistry and precipitation}

There has been little development in the equipment used to sample and analyse precipitation over the past decades, despite the continuing need to quantify wet deposition to ecosystems. There is still no 'perfect' system for sampling and quantifying the chemical composition of precipitation; the best technique is to use 'wet-only' collectors that provide daily (or shorter) time resolution and maintain samples refrigerated to minimize chemical and biological degradation of the collected sample. However, this type of sampler is necessarily bulky and may systematically under-sample small wind-blown droplets. Networks of precipitation samplers more usually use bulk samplers, which also suffer from dry deposition of material onto the collector surfaces between precipitation events, and are in general not protected from degradation by refrigeration or the use of biocides.

Two reviews have recently been conducted in Europe; a largescale field comparison of different (national) sampling systems showed that there is still an unacceptable level of uncertainty in quantifying wet deposition (Erisman et al., 2003), while the different approaches and issues involved in bulk deposition measurement have been reviewed (Dämmgen et al., 2005). Apart from the continuing uncertainties in sampling, it has become clear over the past decade that an important component of the nitrogen cycle has systematically been ignored; water-soluble organic nitrogen (WSON) has been identified as an important component of wet deposition in both marine and continental precipitation (Cornell et al., 2003; Neff et al., 2002), contributing up to one-third of total nitrogen even in regions with significant inorganic nitrogen content (Cape et al., 2004; Liu et al., 2006b).

A new aspect of cloud chemistry concerns the identification of bacteria and fungi as potential agents of aerosol transformation in cloud droplets (Amato et al., 2005, 2007). The application of molecular biology techniques for identifying bacterial strains and 
their impact on organic composition of cloud drops may lead to a new and more integrated vision of the role of biological material on atmospheric chemical cycles.

\subsection{Optical characterization of the atmospheric aerosol}

The impact of atmospheric aerosols on the Earth's climate is driven by their optical properties, namely their ability to absorb and scatter solar radiation in addition to absorbing long-range terrestrial radiation. In situ measurements of aerosol optical properties should therefore allow calculating the direct aerosol climate forcing. The in situ variables used to derive parameters required in the forcing calculation are light absorption, total scattering and backscattering in addition to size, chemical composition and/or refractive index. Techniques for deriving in situ optical properties have been used for many years now but recent developments, mostly based on cavity ring down spectroscopy techniques, are now becoming available and will be reviewed in this section.

\subsubsection{Light scattering and absorption coefficient}

Integrating nephelometers measuring light scattering, and instruments capable of determination of aerosol light absorption coefficients have been commercially available for many years and very few technical advances can be mentioned except, perhaps, the development of polar nephelometers for aerosol and cloud studies (Oshchepkov et al., 2000). As for DMPS/SMPS measurements, the fact that more and more measurements are now available leads to questions concerning the intercomparability of the different instruments. Recently, work has been done to improve the use of nephelometers that are generally limited, due to technical reasons, in forward scattering and backward scattering for angles smaller than $10^{\circ}$ and larger than $170^{\circ}$, respectively. Differences between theoretical illumination - which should ideally follow a sine function - and the real illumination function with its truncation at forward and backward scattering angles have been shown to be significant for some of the commercially available instruments (Müller et al., 2008). Similarly, Heintzenberg et al. (2006) showed that unit-to-unit variability of TSI nephelometers is approximately $10 \%$ for the blue and green wavelengths.

A similar problem to those seen for commercial nephelometers arises for the measurement of the aerosol light absorption coefficient based on the rate of change of transmittance through a fibre filter as particles are deposited (i.e. aethalometers). In fact, the complex relationship between the change in the light transmission and aerosol absorption and scattering optical depth on the filter requires a calibration of these filter-based methods. Calibrations of particle/ soot absorption photometers (PSAP, Radiance Research, Seattle, USA) were developed by Bond et al. (1999) and Virkkula et al. (2005). Correction schemes for aethalometers (Magee Scientific, Berkeley, USA) have been developed by several investigators (Arnott et al., 2005; Schmid et al., 2006; Weingartner et al., 2003), but still show large uncertainties. Some corrections applied to PSAP and aethalometer data require the knowledge of the particle scattering coefficient, often measured with integrating nephelometers. An inherent correction method for minimizing the cross sensitivity to particle scattering was realized by another absorption photometer, the multi-angle absorption photometer (MAAP, Thermo ESM Andersen Instruments, Germany). The MAAP measures the reflectivity of the filter at two angles additionally to the light transmittance. A new generation of photo-acoustic sensors with lower detection limits may set new standards for in situ quantification of soot.

\subsubsection{Cavity ring down spectroscopy}

Cavity ring down (CRD) spectroscopy was originally developed by O'Keefe and Deacon (1988) and became widely used due to its simplicity. A laser beam (pulsed or continuous) is coupled to a cavity that consists of two or three mirrors, and performs a multiple reflection inside the cavity. The intensity of the laser in the cavity is reduced after each pass depending on the reflectivity of the mirror. This produces an exponential decay with a characteristic lifetime. By introducing a sample in the volume of the cavity, the decay becomes shorter due to the additional light extinction. The main advantage of this technique compared to conventional spectroscopic methods is the extremely high sensitivity due to the long path-length (several kilometres depending on the reflectivity of the mirrors). In addition, CRD measurements are insensitive to laser fluctuations. Initially most of the studies were focused on spectroscopy of small molecules in the gas phase, transient molecules in plasmas, radicals, weakly bonded molecules, metal clusters, and observation of weak transitions and kinetics studies. It was also commonly used for detecting trace gases in the atmosphere. In 1988 it was reported that ambient aerosols can considerably decrease the decay time, however, the first application to atmospheric particles was ten years later (Sappey et al., 1998). Table 2 summarizes the work that has been done in the past few years using CRD to study optical properties of aerosols.

The time dependence of the loss of intensity of the laser pulse provides information about the total extinction of the aerosols inside the cavity. CRD has been used to investigate the optical properties of non-absorbing and absorbing aerosol particles by combing it with a DMA and a CPC (Fig. 7) (e.g. Pettersson et al., 2004; Bulatov et al., 2006; Dinar et al., 2008; Riziq et al., 2007, 2008; Spindler et al., 2007). In particular, the system has been used to measure size-dependent extinction from which the complex refractive index and the single scattering albedo of aerosols, a key parameter in modelling the Earth's radiative balance, can be retrieved (Dinar et al., 2008). This method has been used to verify optical properties of mixed aerosols and the applicability of optical mixing rules (Riziq, 2007, 2008), hygroscopic growth of particles (Beaver et al., 2008), and in the field (Baynard et al., 2007). The CRD systems are highly sensitive and can typically measure extinction of $10^{-9}-10^{-10} \mathrm{~m}^{-1}$. The CRD method has been applied mostly at $532 \mathrm{~nm}$, but recent systems have now extended the wavelength range to the near IR and the UV (Dinar et al., 2008).

While most CRD-AS systems are based on pulsed lasers, new systems based on continuous wave (CW) lasers have recently been introduced (Hallar et al., 2006; Lang-Yona, 2009). The Lang-Yona et al. (2009) system uses a single model CW laser which improved the matching between the laser and the cavity modes, hence increasing the sensitivity of the system by about an order of magnitude compared with the pulsed systems. The CW laser-based systems are smaller and consume less power than the pulsed systems hence they have high potential for use in field measurement.

The complex refractive indices of different laboratory-generated particles have recently been retrieved by the CRD-AS. In this method, size selected extinction efficiencies are measured with high precision. Using Mie-scattering calculations, a retrieval algorithm has been developed to obtain the two components of the refractive index. This method has been applied to various organiccontaining aerosols with different types of mixing (homogeneous, core-shell) (Riziq et al., 2007, 2008). This method has recently been implemented for measuring the refractive index of aerosols formed in a smog chamber from emissions of biogenic volatile organic compounds.

\subsubsection{Levitation and optical techniques for single particle analysis}

Analysis of single particles using optical techniques is a field in development and is used by several groups worldwide for obtaining information about properties of atmospheric particles. One of the established techniques in this field is the electrodynamic 
Table 2

Summary of recent studies performed using cavity ring down aerosols spectrometer (CRD-AS) for measurements of aerosols optical properties.

\begin{tabular}{|c|c|c|c|}
\hline Aerosols field of study & Type of CRD & Applications & References \\
\hline Atmospheric particulate matter & $\begin{array}{l}\text { Pulsed-CRD } \\
(532 \text { and } 355 \mathrm{~nm})\end{array}$ & Extinction cross-section of aerosols & Sappey et al. (1998) \\
\hline Soot & Pulsed-CRD (532 nm) & Absorption of soot from methane-air flame & Wal et al. (1999) \\
\hline Ambient aerosols & $\begin{array}{l}\text { Pulsed-CRD } \\
\text { (dual mirrors } 1064-532 \mathrm{~nm} \text { ) }\end{array}$ & Extinction of ambient aerosols simultaneously at two wavelengths & Atkinson et al. (2008) \\
\hline Ambient aerosols & $\begin{array}{l}\text { Pulsed-CRD } \\
(510.2 \text { and } 578.2 \mathrm{~nm})\end{array}$ & Extinction of aerosols during wildfire and local firework & Thompson et al. (2002) \\
\hline $\begin{array}{l}\text { Absorbing and non-absorbing } \\
\text { aerosols }\end{array}$ & $\begin{array}{l}\text { Pulsed-CRD dye laser } \\
(620-640 \mathrm{~nm})\end{array}$ & $\begin{array}{l}\text { Extinction of absorbing and non-absorbing aerosols as a } \\
\text { function of aerosols size }\end{array}$ & $\begin{array}{l}\text { Bulatov et al. (2002), } \\
\text { Bulatov et al. (2006) }\end{array}$ \\
\hline Ambient and laboratory aerosols & $\begin{array}{l}\mathrm{cW}-\mathrm{CRD} \text { at } 690 \mathrm{~nm} \\
\text { and } 1550 \mathrm{~nm}\end{array}$ & $\begin{array}{l}\text { First application of cw-CRD in aerosols studies combined with scattering } \\
\text { detector enable direct measurement of single scattering albedo }\end{array}$ & Strawa et al. (2003) \\
\hline Laboratory aerosols & Pulsed-CRD (532 nm) & $\begin{array}{l}\text { First quantitative study to retrieve complex refractive index of } \\
\text { laboratory-generated aerosols }\end{array}$ & Pettersson et al. (2004) \\
\hline Diesel exhaust & Pulsed-CRD/CED & $\begin{array}{l}\text { First application of CRD and cavity enhanced detection (CED) which limits } \\
\text { the systematic error of CRD }\end{array}$ & Moosmüller et al. (2005) \\
\hline Laboratory aerosols & Pulsed-CRD (532 nm) & The relative humidity influence of aerosols light scattering & Baynard et al. (2007) \\
\hline Laboratory aerosols & Pulsed-CRD (532 nm) & $\begin{array}{l}\text { Determination of complex refractive index and mixing rules of absorbing } \\
\text { and non-absorbing aerosols }\end{array}$ & Riziq et al. (2007) \\
\hline Laboratory aerosols & Pulsed-CRD (532 nm) & $\begin{array}{l}\text { Optical hygroscopic growth of aerosols (humidity dependence of light } \\
\text { extinction of non-absorbing aerosols) }\end{array}$ & Garland et al. (2007) \\
\hline Laboratory aerosols & Pulsed-CRD (532 nm) & Optical properties of coated aerosols (core-shell model) & Riziq et al. (2008) \\
\hline HULIS & $\begin{array}{l}\text { Pulsed-CRD } \\
(532 \mathrm{~nm} \text { and } 390 \mathrm{~nm})\end{array}$ & $\begin{array}{l}\text { Determinations of complex refractive index of Humic Like-Substances } \\
\text { (HULIS) from various sources }\end{array}$ & Dinar et al. (2008) \\
\hline Laboratory and field aerosols & cw-CRD (532 nm) & $\begin{array}{l}\text { CW-CRD designed for field measurements. First application was used to } \\
\text { study secondary organic aerosols formed by oxidation of volatile organic } \\
\text { compounds from plant chamber }\end{array}$ & Lang-Yona et al. (2009) \\
\hline
\end{tabular}

balance (EDB), based on the idea of levitating a charged, single physical entity (an ion, an electron, or a microscopic particle) in a superimposed AC/DC field (see Davis, 1980, 1990, and references therein for an overview). It has numerous applications in the field of atmospheric aerosols since it can be coupled with different investigation techniques like Raman and Mie spectroscopy for the determination of the particle's mass, composition, size and optical properties.

a

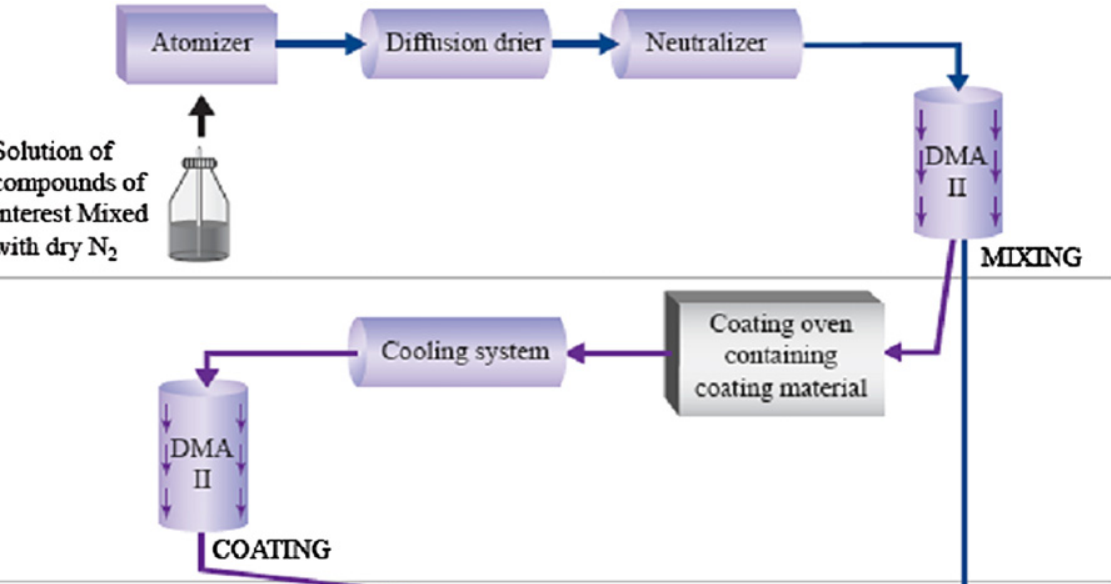

C

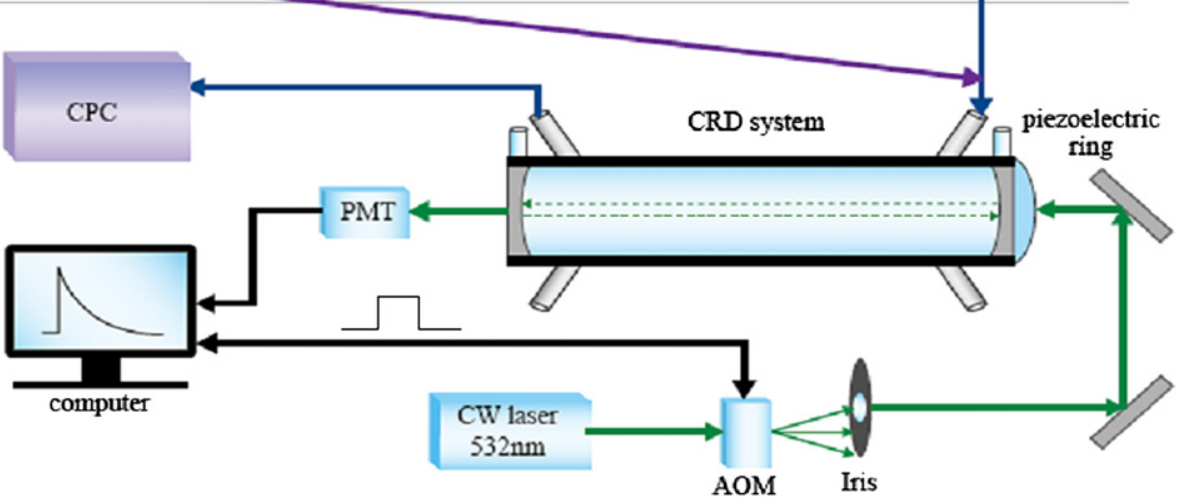

Fig. 7. Schematic of a cavity ring down aerosol spectrometer (CRD-AS) designed for laboratory measurements. The CRD system is combined with an aerosol generator (e.g. atomizer, soot generator etc.) and a DMA for size selection and CPC to determine the particle concentration in the cavity. According to this setup, the extinction efficiency ( $Q_{\text {ext }}$ ) can be determined as a function of size and by using the Mie theory the complex refractive index of aerosols can be retrieved. 
Lately, much effort has been invested in the characterization of compounds recently identified in field measurements, mostly organics of biogenic and anthropogenic origin, and the way they affect aerosol physicochemical properties like hygroscopicity (water uptake), physical state transitions and evaporation. Many studies have clarified the interactions of selected organic aerosols with ambient water vapour (see for instance Peng et al., 2001; Prenni et al., 2003; Choi and Chan, 2002). The natural step further is the study of mixed inorganic/organic aerosol particles; Marcolli and Krieger (2006), Parsons (2006), Zardini et al. (2008), Ling and Chan (2008), Lee et al. (2008) and Treuel et al. (2008) measured the hygroscopicity and/or phase transitions for several ternary inorganic/organic/water systems, while Chan and Chan (2007) investigated the mass transfer limitation at the gas/particle interface.

Optical techniques have been developed and tested on levitated aqueous solution droplets containing organic material to infer very low vapour pressures from measured evaporation rates (Zardini et al., 2006). Other studies focused on ice nucleation (Duft and Leisner, 2004) and mass accommodation coefficient determination (Magee et al., 2006; Xue et al., 2005; Zientara et al., 2005). Besides the electrodynamic levitation, an aerosol particle, or an array of particles, can be trapped and characterized by means of optical tweezers (Hopkins, 2004; Hanford et al., 2008). Cavity enhanced Raman scattering has also been used to characterize the evolving composition of evaporating ternary solution droplets (Howle et al., 2007) falling as a droplets train generated with a vibrating orifice.

\subsection{Airborne aerosol measurements}

Although measurements of aerosol particles from aircraft have been performed since the middle of the 20th century, the available data are very limited, particularly in the free troposphere. This is because research aircraft were most often used, which yielded information only over limited time and space. Furthermore, in many cases only physical aerosol parameters were determined and chemical information was limited mostly to filter samples with coarse time resolution. At the turn of the millennium, the situation changed slowly as longer data sets became available (Moore et al., 2004) and new technologies were applied.

\subsubsection{Improvements in aerosol sampling technology}

The development of aircraft sampling technologies has benefited from advances in aerosol sampling methodologies. In particular, the use of computational fluid dynamics (CFD) codes became more powerful and easier to use (e.g. Krämer and Afchine, 2004; Eddy et al., 2006). The design or characterization of highly sophisticated inlets could be realized using these CFD tools (Dhaniyala et al., 2003, 2004; Wilson et al., 2004). A number of research aircrafts are now equipped with well defined aerosol inlets. Probably the most important new aircraft-borne inlet constructed since about 2000 is the Low Turbulence Inlet (LTI) of the University of Denver (Wilson et al., 2004) shown in Fig. 8. By applying boundary layer suction, turbulence inside the inlet is reduced to nearly zero, which allows in particular super-micrometre particles to pass the inlet with high efficiency.

Besides CFD modelling, wind tunnel experiments are an appropriate way to quantify the inlet sampling efficiency. Similarly, inter-comparisons in-between aircraft-borne measurements and between aircraft-borne and ground-based measurements are well suited for that propose. Fortunately, this kind of comparisons were funded and conducted more strongly in recent years (Blomquist et al., 2001; Hegg et al., 2005; Moore et al., 2004).

\subsubsection{In situ aerosol measurements from airborne platforms}

One of the most important technical achievements in the last decade remains the CARIBIC project (Civil Aircraft for Regular Investigation of the Atmosphere Based on an Instrument Container, http://www.caribic-atmospheric.com). In the project, particle number concentrations and particle elemental composition are measured regularly by an instrument container onboard a commercial aircraft (Brenninkmeijer et al., 2007). The CARIBIC data give for the first time in situ information on aerosol particles in the upper troposphere on nearly global scale (Heintzenberg et al., 2003; Martinsson et al., 2001, 2005; Nguyen et al., 2008; Papaspiropoulos et al., 2002; Weigelt et al., 2009). Another important recent achievement has been the use of AMS techniques onboard research aircraft. The sensitivity together with the fast time resolution of AMS techniques makes it a very suited instrument for airborne platforms. In particular, the development and application of a particle laser mass spectrometer (PALMS) yielded new insight into particle chemistry, in particular the hitherto underestimated contribution of organics to particle material in the free troposphere (Murphy et al., 1998, 2006a,b, 2007).

\subsubsection{Remote sensing from airborne platforms}

Following successful ground-based measurements of particle extinction (Hair et al., 2001), high spectral resolution lidar (HSRL) is

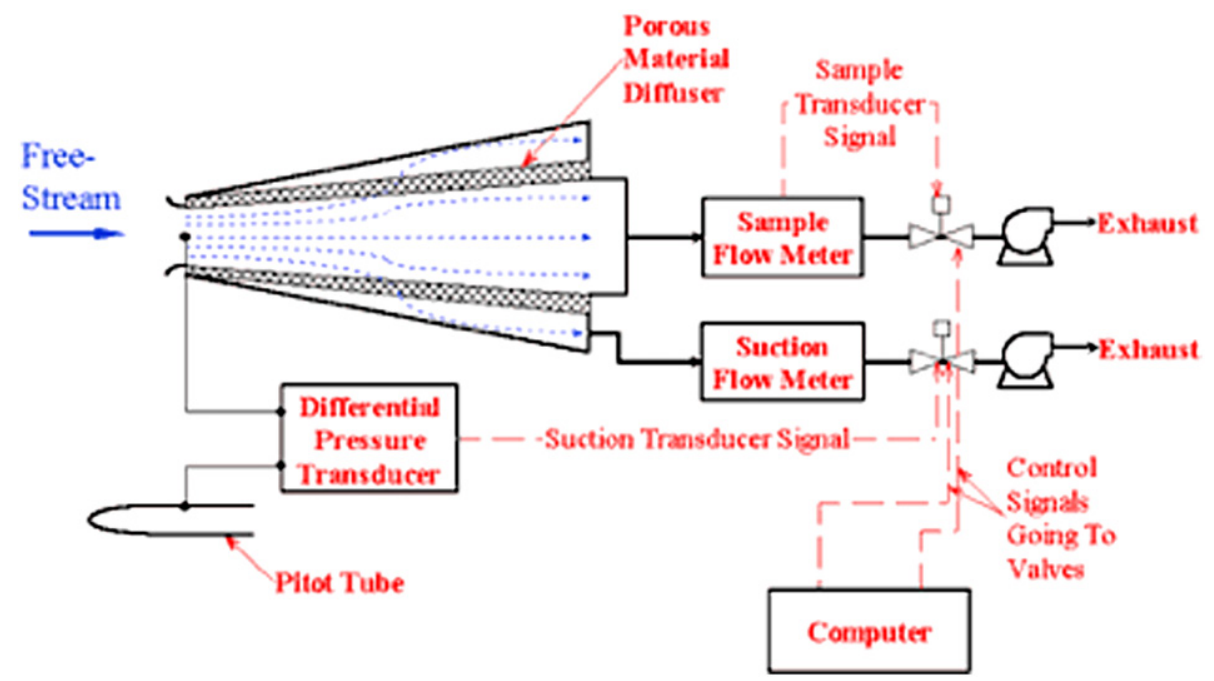

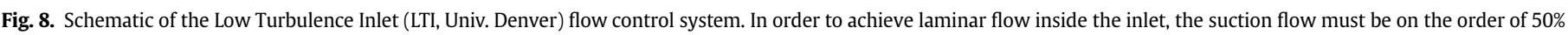
or more of the total flow (Seebaugh and Wilson, 1999). 
now used onboard airborne platforms. HSRL works on the basis of spectral broadening of light backscattered by molecules and aerosols. This spectral broadening is caused by Doppler broadening due to thermal motion, and it is different for molecules and aerosols. An HSRL separates the radiation scattered by molecules from the radiation scattered by particles and allows the calculation of particle extinction coefficients. The first high-quality profiles of particle extinction coefficients were acquired in the late 1990s (Wandinger et al., 2002). At that time it was shown how one can roughly distinguish different particle types. An HSRL next to a Raman lidar allows for direct measurements of the particle lidar ratio. As was shown by Müller et al. (2007a,b) one can roughly distinguish particle types if the lidar ratio at one wavelength is measured. The instruments have been successfully improved for airborne operation (Esselborn et al., 2008). Most notably, extensive profiling of mineral dust optical properties was carried out in 2006 and 2008 as part of the Saharan Mineral Dust Experiments (SAMUM) (Esselborn et al., 2009). Successful measurements have begun recently as part of NASA's first space-borne lidar aboard the CALIPSO satellite (Liu et al., 2008). The ongoing work is concerned with upgrading the HSRL to full multi-wavelength systems. Particle extinction measurements at two wavelengths (355 and $532 \mathrm{~nm}$ ) will not only provide lidar ratios but also Ångström exponents.

\subsection{Remote-sensing aerosol characterization from the ground}

Detailed knowledge of optical, microphysical, and radiative properties of aerosol particles is required to understand their role in atmospheric processes as well as their impact on human health and the environment. To fully characterize aerosol particles it is necessary to measure their optical properties, size, morphology and composition as a function of time and space, possibly with high spatial and temporal resolution to account for the aerosol variability. On the one hand, information can be derived from passive remotesensing techniques such as sun - and sky - photometers and, on the other hand, from active remote sensing using lidar type instruments.

\subsubsection{Passive remote-sensing techniques}

2.8.1.1. Sun photometers. Most of the aerosol information retrieved by passive remote-sensing techniques has been obtained through AERONET (AErosol RObotic NETwork, http://aeronet.gsfc.nasa.gov/). AERONET is a federation of ground-based remote-sensing aerosol networks and was established in the mid-90s by NASA and CNRS with further expansion through national agencies, universities and individuals (Holben et al., 1998). With standards for instruments, calibration techniques, processing and data-distribution, individual sites are inter-connected into a network. Currently more than 150 instruments are simultaneously in operation worldwide. Since many instruments have been moved during their lifetime, column data are available from many more sites, however long-term measurements are relatively scarce. Although now more than 700 sites are in the database, the number of sites with quality-assured monthly averages (50 + attenuation sun samples or $10+$ sky radiance samples) and covering at least 10 different months, amount to 370 (220 sites with sun data and 150 sites with sky data).

The basic instrument is a CIMEL sun-/sky-photometer. Data are transmitted via satellite or internet to the central processing facility at NASA, where the retrieved products from almost all sites are available within hours. Quality assured products are released at a later time after the instruments have been recalibrated. Retrieved aerosol products are column properties for (1) aerosol optical depth (AOD) at several wavelengths of the solar spectrum (380, 440, 500, 670, 870 and $1020 \mathrm{~nm})$, which define the Ångström parameter, (2) size distribution and (3) absorption. The products for size and absorption are derived from the application of sampled sky-radiances (at 440,500, 670 and $870 \mathrm{~nm}$ ) in an inversion algorithm (Dubovik and King, 2000), with the caveat that absorption estimates are only reliable if the mid-visible spectrum AOD value exceeds 0.3.

The appealing nature of AERONET is that all aerosol (column) properties are addressed without fixed a priori assumptions, that retrievals have a high accuracy as they (cirrus events excluded) do not suffer from background issues, and that data from different AERONET sites are inter-comparable. The AERONET data provide valuable constraints to complementary retrievals for a priori assumption as in the case of satellite sensor data or for co-located elastic backscatter lidar systems.

The processing algorithms have evolved from Version 1.0 to Version 2.0 (Holben et al., 2006). Overall, this update resulted in only minor changes to the overall statistics. Other unofficial AERONET aerosol data products include estimates of the fine-mode AOD fraction based on the solar spectral dependence of the Ångström parameter (O'Neill et al., 2003) and estimates of aerosol water content and soot mass from AERONET derived refractive indices (Schuster et al., 2005). In addition, a special (though rarely applied) downward looking detector mode (during aerosol sampling breaks) over vegetation (taking advantage of surface reflectance differences in the solar and near-infrared regions) allows for the retrieval of cloud liquid water and cloud optical depths (Marshak et al., 2004).

In a recent ground-based study it has been shown that the area assumed to be cloud-free within a cloud field is filled with forming and evaporating clouds, undetectable small clouds and hydrated aerosols. This area, defined as the 'twilight zone', has unique optical properties that are currently missed by most observations due to binary classification of the atmosphere as either cloudy or cloud-free (Koren et al., 2007). The 'twilight' properties, when averaged over the coarse pixel size create an apparent gradual transition from a cloudy to a cloud-free atmosphere. However, when zooming in, the zone emerges as non-uniform, and affected by many distinct processes (Koren et al., 2008). The 'twilight' properties are strongly linked to the aerosol loading, suggesting an additional aerosol effect on the composition and radiation fluxes of the atmosphere.

When analysing the 'twilight' from satellite data, other processes and instrumental artefacts may contribute to the enhanced reflectance in the vicinity of clouds. For example, photons escaping from the side of the cloud scattered towards the satellite by air molecules and aerosols may result in higher reflectance near clouds (Wen et al., 2006). Although these effects happen at a spatial scale much smaller than that is measured in the 'twilight zone', measuring the 'twilight' properties from the surface using AERONET data from ground-based sun-photometers (Holben et al., 1998) gives a cleaner view of the phenomena. Artefacts caused by nearcloud scattering will increase the measured photon flux and therefore will result in an underestimation of the 'twilight' optical depth, and the optical collimator on the AERONET instrument reduces stray light artefacts. The AERONET instruments collect data at intervals no longer than $15 \mathrm{~min}$, unless a cloud blocks the direct view of the sun. The absence of a sun measurement at the appointed times can be used as a proxy for the presence and the coverage of clouds (Kaufman and Koren, 2006).

Complementary AOD measurements to AERONET sampling are provided by the precision filter radiometer (PFR) (Wehrli, 2000). This instrument has a much higher sampling rate and is especially suited for very low values of AOD and is mainly used at sites of the Global Atmosphere Watch (GAW) programme of WMO. Comparisons of AODs determined from AERONET CIMEL and GAW PFR at the high altitude site Mauna Loa showed an excellent agreement with 0.001 bias for $500 \mathrm{~nm}$ AOD (Kim et al., 2008). 
2.8.1.2. Multi-axis differential optical absorption spectroscopy. The Multi-Axis Differential Optical Absorption Spectroscopy (MAX-DOAS) is a newly introduced passive remote-sensing technique for monitoring the vertical distribution of aerosol properties. Most of the ongoing MAX-DOAS research investigations focus on the retrieval of aerosol properties based on $\mathrm{O}_{4}$ and intensity measurements that are performed simultaneously with the trace gases measurements (Heckel et al., 2005; Wagner et al., 2004; Frieß et al., 2006). This aerosol retrieval step is essential for the accurate determination of trace gas profile information down to the surface.

\subsubsection{Active remote-sensing techniques for aerosol studies from the ground}

Lidar techniques are ideal for collecting range-resolved data on aerosol particles. The basic lidar system for acquiring data on aerosol particles is the elastic backscatter lidar. Such lidars allow for the direct determination of the geometrical thickness of aerosol layers and the altitude at which they occur. However, this type of lidar is not able to provide independent measurements of the particle backscatter and particle extinction coefficients. The Raman lidar technique overcomes this difficulty as it provides independent aerosol extinction measurements (Ansmann et al., 1990). The technique has been widely and successfully used for aerosol extinction measurements (Pappalardo et al., 2004; Mona et al., 2006; Mattis et al., 2008); nowadays the Raman lidar technique is extensively used in EARLINET (European Aerosol Research Lidar Network) [www.earlinet.org] and at the ARM Site in US (Turner et al., 2002). Further developments in lidar instrumentation and, in particular, advances in multi-wavelength Raman aerosol lidar techniques have been demonstrated to have the unique ability of providing range-resolved aerosol microphysical properties (Ansmann and Müller, 2005; Böckmann et al., 2005; Müller et al., 1999; Veselovskii et al., 2002). Advances in lidar technology have been made in parallel with the development of data inversion algorithms. For a historical overview on the change from a non-applicable methodology to current successful algorithms see Ansmann and Müller (2005). The capability of the lidar technique is illustrated in Fig. 9, showing optical profiles of a forest-fire smoke plume observed with a high performance multiwavelength Raman lidar. The profiles of the particle backscatter coefficients and extinction coefficients are extensive parameters, as they depend on particle number concentration. The profile of the
Ångström exponent is height independent in the present example and can be calculated from the profiles of the extinction coefficients (measured at two wavelengths). We can also determine the socalled backscatter related Ångström exponents, which follow from the profiles of the particle backscatter coefficients. The Ångström exponents are independent of particle concentration, but depend for instance on particle size. The particle lidar ratio, which is another intensive property, is larger at $532 \mathrm{~nm}$ than at $355 \mathrm{~nm}$. This wavelength dependence is characteristic for long-range transported forest-fire smoke.

Despite the successful application of inversion methods, strong limitations still exist regarding the retrieval of aerosol particle microphysical parameters. Even modern multi-wavelength Raman lidars provide data at a few measurement wavelengths only. That limitation severely restricts the number, type, and accuracy of the retrievable microphysical parameters. The "exact" shape of the particle size distributions generally cannot be retrieved. The parameters that are usually used to describe such size distributions are mode radius, and geometrical standard deviation (mode width) if we use logarithmic-normal particle size distributions. These two parameters usually cannot be derived with acceptable accuracy. In general we may only estimate whether the acquired data describe a mono- or bimodal particle size distribution. The maximum size of particles that can be derived is limited, too. It has been shown (Veselovskii et al., 2005) that particle effective radii of around $2 \mu \mathrm{m}$ are retrievable, if extreme care in data analysis is taken. In view of the need for operational algorithms this number has to be reduced to an effective radius of less than $1 \mu \mathrm{m}$, which puts the retrievable particle size distributions into the so-called fine-mode fraction of the particle size distribution.

Multi-wavelength Raman lidar observations cannot be used directly to infer the presence and/or concentration of water-soluble and water-insoluble components. They cannot distinguish between, for example, sulphate-containing particles from nitrate-containing particles. This means that a characterization of the particles in terms of chemical components is impossible. Rather, the primary products of lidar retrievals are the average values of particle size and complex refractive index. These values then may be attributed to the likely presence of certain particle components such as small anthropogenic particles. Evidence for sulphate or nitrate can be made with the complex refractive index, using the aforementioned inversion algorithms. Determination of particle origin by lidar
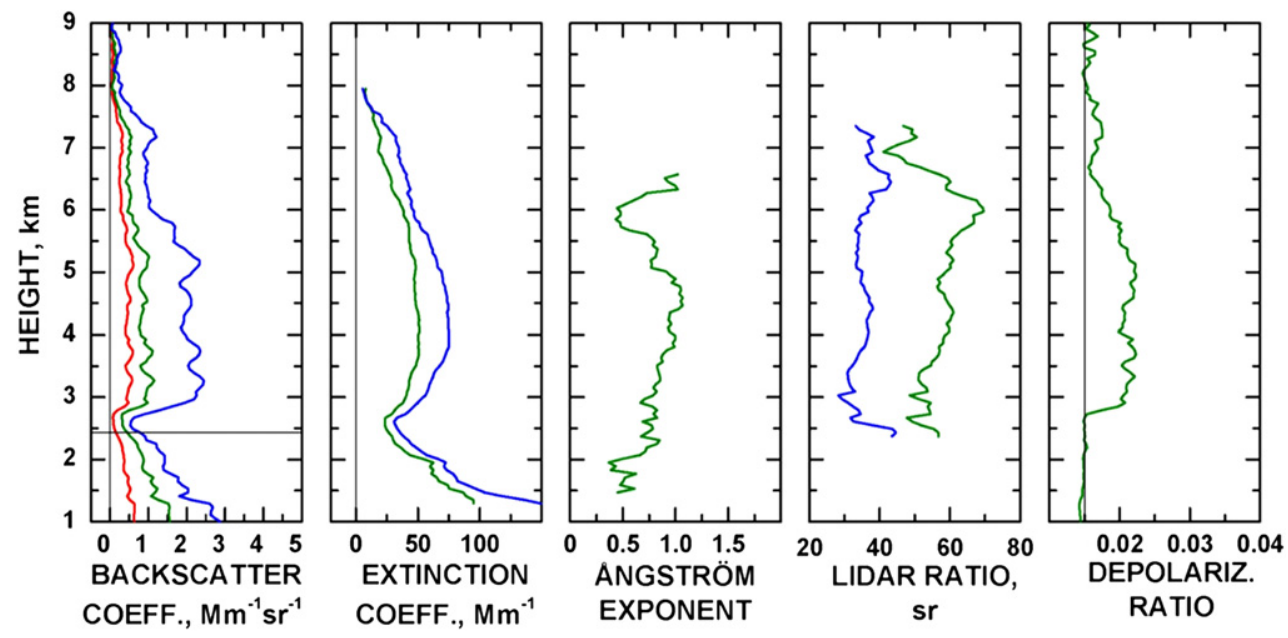

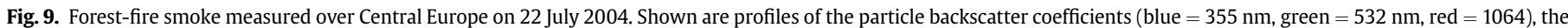

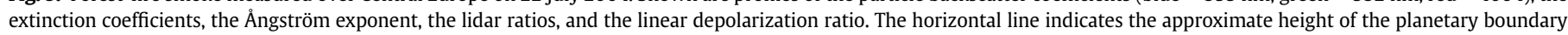
layer. The example is discussed by Müller et al. (2007a,b). 


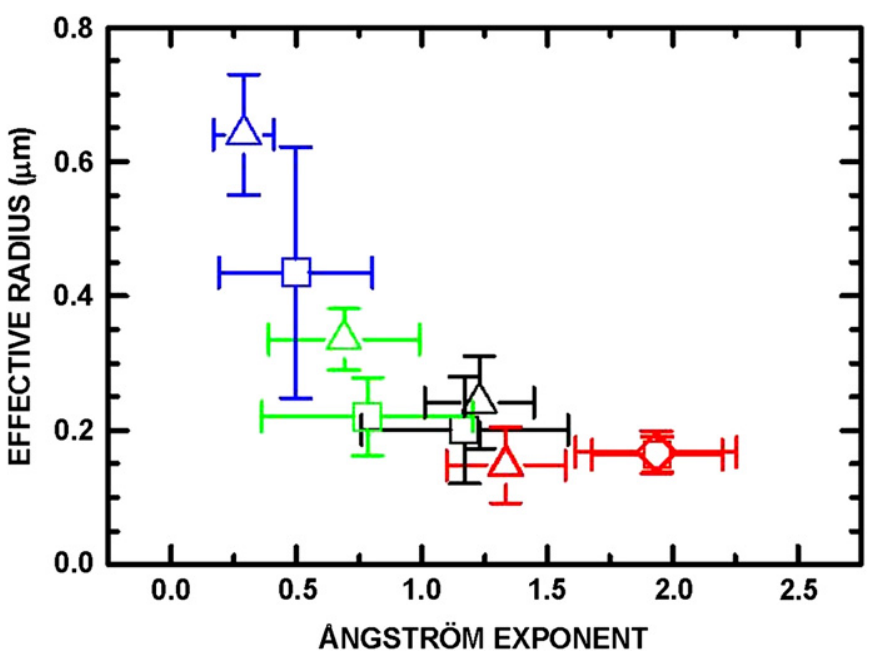

Fig. 10. Effective radius and Ångström exponents of different aerosol types. Shown are the following results: anthropogenic pollution from North America (red square) and Europe (red triangle), Arctic haze/urban pollution from polar regions north of $70^{\circ} \mathrm{N}$ (red diamond), mixtures of anthropogenic/biomass-burning particles from South Asia (black square) and East Asia (black triangle), Canadian and Siberian forest-fire smoke observed over Europe (green triangle) and Spitsbergen (green square), marine particles in the North Atlantic (blue triangle) and the Indian Ocean (blue square). The error bars denote the variation of the values obtained for the two parameters from various measurements, i.e. measurement errors are not included. The results for the marine particles describe data of lidar observations in the upper parts of the marine boundary layer.

technique has been applied by Müller et al. (2005, 2004). Saharan dust particles may easily be identified by measuring the particle depolarization ratio with depolarization ratios larger than $10 \%$ (at $532 \mathrm{~nm}$ wavelength), higher than in any other particle type. Fig. 10 summarizes the results of 10 years of observations with multiwavelength aerosol Raman lidar over Leipzig $\left(51.3^{\circ} \mathrm{N}, 12.4^{\circ} \mathrm{E}\right)$, Germany, (Mattis et al., 2008) and results of field campaigns in which multi-wavelength Raman lidar played an important role.

\subsubsection{Ground-based active remote-sensing techniques for cloud studies}

The phase composition and microphysical structure of clouds define the radiative impact of clouds on atmospheric radiation and their contribution to the hydrologic cycle (Shupe et al., 2008; Stephens et al., 1990; Baker, 1997). To retrieve these cloud properties, measurement of cloud phase and boundaries, ice properties, liquid water path, water vapour amount, optical depth and vertical velocity are needed. Extensive ground-based lidar measurements have been used for the investigation of clouds (Feingold and Grund, 1994; Sassen and Mace, 2002). Lidar remote sensing of clouds is traditionally considered limited to clouds with a low liquid water amount, i.e. thin clouds with an optical depth less than 3. But in the last few years, both the use of novel observation techniques and technological evolution has motivated the use of lidar in cloud studies. Several lidar techniques have been applied for the investigation of water vapour, liquid water, cloud droplet and ice crystal sizes.

Lidar measurements of atmospheric depolarization allow identification of the cloud phase on the basis of the sensitivity of particle depolarization on particle shape (Sassen, 2000). The phase discrimination of the cloud ice/water phase is performed using the ratio of signals in two orthogonal polarization receiver channels. However, lidars used in atmospheric research do not penetrate directly most boundary-layer clouds due to their large optical density. Measurements are typically limited to provide information up to the first interface (i.e. ceilometry from ground), though the use of depolarization can also provide information about the water phase in the first layers. Nevertheless, at most visible and near-IR wavelengths, photons from lidar are not absorbed but merely scattered out of the beam. Therefore, a significant increase in information that can be obtained from lidar observations is related to the use of multiple-field-of-view (MFOV) Raman lidar techniques (Bissonnette, 1995; Malinka and Zege, 2007). With the application of these techniques for routine cloud profiling, it is possible to derive effective cloud droplet size in the lower parts of the clouds. The MFOV technique uses the multiple scattering contributions to the lidar signal to retrieve the range-resolved extinction coefficient and effective droplet diameter. From the latter two parameters we may infer microphysical products like the liquid water content (LWC). Similarly, techniques using lidars at different wavelengths and elevation angles of the lidar beam can be applied to characterize the size of the ice particles (from the ratio of the lidar backscatter at different wavelengths) and their shapes and habits (from the linear depolarization ratio(s) and backscatter coefficient(s) at zenith and off zenith direction of the laser beam).

Improved results in cloud monitoring can be obtained by using the available instruments in synergy, either by combining active sensors at different wavelengths or by augmenting the active sensors with passive radiometric measurements. For example, the geometry and vertical location of cloud layer with different phases in stratiform clouds can be achieved using a multi-sensor approach that takes advantage of the combination of lidar, radar and microwave measurements. Microwave radiometers are able to provide accurate estimates of the liquid water path also in supercooled environments. The temporal resolution can be of the order of $1 \mathrm{~s}$ (Crewell et al., 2001; Kämpfer et al., 2006) and the accuracy is better than $20 \mathrm{~g} \mathrm{~m}^{-3}$ (Hewison, 2006). In addition, microwave radiometers also provide information about the vertical distribution of liquid water even though liquid water profiling in mixed-phase clouds is very complicated. Moreover the scanning capability of microwave radiometers permits us to apply microwave tomography which can be used for characterization of the three-dimensional distribution of clouds. Accurate liquid water path estimates are provided also by AERI (Atmospheric Emitted Radiance Interferometer) whose sensitivity and accuracy is higher than microwave radiometer sounding in optically thin cloud conditions (Turner, 2007). The merging of Doppler spectral measurements and dual-polarization observations allows the determination of the microphysical properties of mixtures of different ice crystals in the investigated air volume, i.e. crystal habits and particle size distributions (Gaussiat et al., 2003; Russchenberg et al., 2005).

The combination of the information provided by these sensors delivers physically consistent profiles of cloud parameters, such as water content, particle size, and optical extinction through suitable retrieval algorithms. The synergetic products obtained from integrated approaches also help us to address the cloud impact on climate, both in the shortwave and long-wave portion of the electromagnetic spectrum. Instrument synergies are essential for the calibration/validation of satellite measurements as well as for the evaluation of atmospheric models (Löhnert et al., 2008).

Many different integration approaches have been developed. For example, lidar-radar techniques are particularly useful for thin clouds (Donovan and van Lammeren, 2001). A first attempt to develop an all-encompassing algorithm for cloud retrieval based on optimal estimation retrieval is presented by the Integrated Profiling Technique (IPT, Löhnert et al., 2004). IPT combines information from radar, lidar and microwave profiler. Moreover, the retrieval algorithms developed as part of Cloudnet (www.cloud-net.org) are a valuable example of the integration of ground-based measurements from active and passive sensors and an NWPM (Numerical Weather Prediction Model), including an accurate cloud classification scheme (Illingworth et al., 2007). 


\subsection{Observations of tropospheric aerosol from space}

\subsubsection{Past and current space-borne sensors}

Aerosol satellite remote sensing started using a geostationary satellite with a single wavelength and a single angle of observation (Fraser et al., 1984; Jankowiak and Tanré, 1992; Husar et al., 1997). A two-channel technique was also used for the AVHRR with simultaneous analysis of aerosol and clouds (Nakajima et al., 2001; Sekiguchi et al., 2003; Mishchenko et al., 2003). These instruments, designed for monitoring surface and meteorological fields, provide aerosol information but over water only, when the surface is dark. In the UV part of the solar spectrum the land reflectance is very low and data can be also used directly to observe aerosol over land and ocean. The TOMS instruments (including OMI on AURA) have two UV channels that are suitable for observations (Herman et al., 1997) of elevated smoke or dust layers above the scattering atmosphere. These pioneer instruments supply a long-term series that can be used to assess regional changes in, for example, the aerosol concentration but the number of retrieved aerosol parameters is limited.

New space-borne instruments use the multi-dimensional (i.e. spectral, angular \& polarization) signature of sunlight scattered by the atmosphere to derive the global distribution of aerosol column concentration, and several microphysical properties, like size or shape and refractive index. The spectral information is used, for example by the MODIS instrument on NASA's Terra satellite launched in 1999 (King et al., 1992), or by MERIS on the European Space Agency's Envisat satellite launched in 2002. Over the ocean, MODIS algorithms use the aerosol spectral signature in a wide range $(0.47-2.1 \mu \mathrm{m})$ to distinguish small particles, which in high concentrations are typically associated with anthropogenic pollution or smoke, from coarse particles usually identified as natural sea salt or dust. Over land, the transparency of fine mode smoke or urban aerosol in the $2.1 \mu \mathrm{m}$ channel is used to observe the surface cover properties, to estimate surface reflectance at visible wavelengths, and to derive the aerosol optical thickness from the residual reflectance at the top of the atmosphere (Remer et al., 2005).

Angular and spectral information is provided by POLDER, MISR and AATSR to derive, in addition to the concentration, the aerosol type and to increase the sensitivity to aerosol over bright land surfaces. MISR (Diner et al., 1998) measures the reflected light at different viewing angles (nadir and up to $70^{\circ}$ forward and backward) along the satellite's track and in a narrower spectral range $(0.44-0.86 \mu \mathrm{m})$ than MODIS. The inversion technique is then able to separate the aerosol signal from that of the surface, and to derive information about particle size and shape (Kahn et al., 2001). A mixed approach using two viewing directions but over a wider spectral range $(0.55-1.65 \mu \mathrm{m})$ is used by AATSR (Veefkind et al., 1998) to derive the aerosol concentration and type.

The POLDER instruments (Deschamps et al., 1994) deployed on the ADEOS-I, ADEOS-II and on the PARASOL satellites, use a combination of spectral channels in a range similar to MISR $(0.44-0.91 \mu \mathrm{m})$. The instruments are wide-angle cameras observing the same target on the Earth at up to 15 different angles. They offer the unique capability of measuring polarization, which yields additional insight into aerosol optical properties beyond aerosol optical thickness over ocean (Herman et al., 2005). The light polarization can also be used to detect fine aerosols over land, taking advantage of the small difference between the spectrally neutral polarized light reflected from the Earth's surface and the spectrally decreasing polarized light reflected by fine-mode aerosols (Deuzé et al., 2001).

The retrieval of aerosol properties from space has improved greatly over the past decade. The MODIS and MISR instruments (initially launched in late 1999 on NASA's Terra satellite) provide information about aerosol abundance and properties at high spatial resolution. They provide considerable insight into the global distribution of aerosols, their long-range transport, and their optical properties. Further insight into the spatial distribution of aerosol species was recently derived from PARASOL (launched on December 18, 2004 and routinely acquiring data since March 2005). The PARASOL (Polarization and Anisotropy of Reflectances for Atmospheric Science coupled with Observations from a Lidar) payload is largely based on the POLDER instrument (Deschamps et al., 1994) and contributes to a better knowledge of the optical, physical and radiative properties of clouds and aerosols. The large wavelength range of SCIAMACHY is also ideally suited for the detection of clouds and aerosols (von HoyningenHuene et al., 2007; de Graaf and Stammes, 2005). More recently, the OMI instrument (Levelt et al., 2006) on AURA and the GOME2 (Callies et al., 2000) on MetOp add to the UV/visible data record at high spatial resolution and with improved coverage. In addition, OMI measures aerosol absorption and cloud scattering layer mean pressure. Finally, the Cloud-Aerosol Lidar and Infrared Pathfinder Satellite Observations (CALIPSO) were launched on April 28, 2006 for a planned 3-year mission. CALIPSO is flying in formation with the AQUA, AURA, CloudSat, and PARASOL satellites as part of the Afternoon Constellation or A-train. The CALIPSO payload consists of three instruments: the Cloud-Aerosol Lidar with Orthogonal Polarization (CALIOP); the Infrared Imaging Radiometer (IIR) and the Wide Field Camera (WFC). CALIOP is a nadir-pointing instrument which provides information on the vertical distribution of aerosols and clouds as well as their optical and physical properties.

The amount of satellite remotely sensed data devoted to aerosols has increased greatly in the past ten years. The accuracy of the instruments, as well as the quality of the retrievals, has been improved and we are probably at the peak of research benefits from these recent satellite sensors. New instruments that combine a wide spectral range with a wide viewing angle range and with polarization sensitivity are proposed for future missions in the 2015-2020 time frame. A pioneer instrument is going to be launched in 2009 (Mishchenko et al., 2007); it is not an imager that allows global coverage but it is expected to improve the determination of aerosol size and composition, through the determination of the refractive index, along the satellite ground track.

\subsubsection{Space-borne lidar for aerosol studies}

The high variability both in space and time of tropospheric aerosols is the main cause of the high uncertainty about radiative forcing related to tropospheric aerosols and their interactions with clouds (Forster et al., 2007). In the past, the variability of the horizontal and temporal distribution of aerosols and of their optical properties has been largely investigated at a global scale by means of passive remote sensing instruments aboard satellites or groundbased sun photometer networks like AERONET (Kaufmann et al., 2000; Omar et al., 2005; Kahn et al., 2007).

The first aerosol measurements from space were a qualitative index, the aerosol index, provided by TOMS (Herman et al., 1997), and the optical depth at $630 \mathrm{~nm}$ provided over the oceans by AVHRR (Husar et al., 1997) both available since the late seventies. The accuracy of aerosol integrated optical depth measurements greatly improved with the coming of MODIS (Kaufman et al., 1997) and MISR (Diner et al., 1998) instruments, launched in 1999, thanks to the higher spectral and spatial resolutions. However these instruments cannot provide information about the vertical layering of aerosols and this vertical distribution is a crucial point for aerosol-clouds interaction.

The first experiment with lidar in space was the LITE (Lidar Inspace Technology Experiment) mission, an 11-day mission carried out in 1994 (McCormick et al., 1993). Although it was a very 
short-time experiment, LITE provided for the first time a snapshot of the atmospheric layering on large scales and paved the way for the current and future space-borne lidar missions. Since April 2006, CALIPSO, the first satellite-borne lidar specifically designed for aerosol and cloud studies, has been providing high vertical resolution profiling of aerosol and clouds on the global scales (Winker et al., 2007). Since its launch, CALIPSO has provided a near-continuous global data set of high-resolution vertical profiles of aerosols and clouds, allowing for the inspection of stratospheric and free-tropospheric layer dynamics (Liu et al., 2008) and the investigation of complex effects of mixing processes that influence the microphysical and optical properties of aerosols and clouds. In addition, flying in the A-train constellation, CALIPSO offers, for the first time, the possibility for developing an integrated strategy between lidar and passive remote-sensing techniques with the synergies among different sensors for both aerosols and clouds studies (Stephens et al., 2002; Anderson et al., 2005; Hu et al., 2007; Lamquin et al., 2008; Sassen et al., 2008).

The CALIPSO cloud and aerosol observations data set will be virtually extended by the ESA's Atmospheric Dynamics Mission (ADM-Aeolus) (Stoffelen et al., 2005; Ansmann et al., 2006), the Earth Clouds Aerosols and Radiation Explorer (EarthCARE) mission of ESA and the Japan Aerospace Exploration Agency JAXA (ESA, 2004). The 3-mission observations will provide a unique 10 -year long data set on the global aerosol and cloud distribution and respective trends in cloud cover, cloud amount, and aerosol pollution state. Such long-term global aerosol records are needed in order to quantify the direct climate forcing by anthropogenic aerosols as well as the indirect aerosol effects on climate and to improve numerical weather prediction, climate research, and future climate prediction.

\section{Measurement of the atmospheric gaseous composition}

The Earth's atmosphere is a complex mixture of many gases of which most are present as trace amounts at sub-ppb levels but still sufficient to play a major role in atmospheric chemistry at regional and global levels. It is clear that the gaseous composition of the atmosphere is changing due to both natural and anthropogenic influences leading to changes of the oxidative capacity of the atmosphere and consequences on air quality (see Monks et al., in this issue; Isaksen et al., in this issue; Fowler et al., in this issue). Significant advances have been made in the major fields of atmospheric research with respect to (i) studies to improve our understanding of chemical processes, (ii) the global distribution and trends of trace gases, and (iii) the integration of observations from various platforms. The inventories of trace gas emissions have been substantially improved in recent years in particular with regard to aerosols and ozone precursors, both of which have implications for climate as well as for air quality in the lower atmosphere. Similarly, our knowledge on chemical reactivity of many atmospheric gases has been considerably improved, particularly for numerous volatile and semi-volatile organic carbon compounds present in the atmosphere. Clearly, the interaction of many individual sources emitting their own particular range of chemical compounds and their extremely complex reactivity leads to an atmospheric cocktail that requires sophisticated sampling and analytical techniques for speciation and quantification of each component. As for the previous chapter related to aerosol studies, this chapter is sub-divided between in situ techniques and remote-sensing techniques from the ground and from space. An overview of current findings and fields of research is provided for controlled conditions in laboratory and in situ studies, emphasizing the basic new technical developments that enabled or supported recent scientific advances. First, this is presented in general and then detailed for the respective groups of trace gases measured.

\subsection{In situ characterization of gaseous atmospheric composition}

In recent years, significant breakthroughs in measurement technique have been made with respect to developments in mass spectrometry coupled to new and selective ionisation techniques, and cavity based optical absorption spectroscopy partly associated with new light sources. Some general descriptions concerning these new techniques are given here. Applications of these new techniques and additionally of optical absorption in the liquid phase (long path absorption photometer, LOPAP), photo-acoustic sensors, vacuum-UV resonance fluorescence, and thermal dissociation laserinduced fluorescence (LIF) are given in the compound by compound section below. In this whole area of developments of advanced measurement techniques, challenges for available techniques are the extremely low concentrations, high reactivity of the compounds of interest, and the aimed accuracy in the range of $10 \%$ or better. Deviations between measurements and models may always, to some extent, be due to yet unknown technical uncertainties and artefacts, and thus there is a high demand for more inter-comparisons between ideally independent techniques covering the range of atmospheric composition variability. The focus in this section is, therefore, on technical improvements enabling and contributing to new types of process studies, especially radical chemistry, and studies to understand the processing of mainly anthropogenic emission plumes by detailed observations of $\mathrm{VOC}$ and $\mathrm{NO}_{\mathrm{y}}$ components (see Monks et al., in this issue). In addition, emphasis will be given to quality assurance issues and results from inter-comparison exercises that are a crucial part of the measurements.

The central player in the oxidation chemistry of the atmosphere is the $\mathrm{OH}$ radical and with the development of more sensitive and time-resolved techniques for the detection based on laser-induced fluorescence (LIF) and selective atmospheric pressure chemical ionisation mass spectrometry (often referred to as CIMS) in the 1990s, numerous studies of OH chemistry in various environments have been performed. The results are often in agreement with current scientific understanding, e.g. model results, however, also deviations exist mainly for night-time observations, in strongly forested and in urban environments (Monks et al., in this issue). These deviations will be the focus of future research activities and it has to be shown that instrumental problems contributing to such deviations are well characterized and suppressed.

Understanding of the budget of $\mathrm{HO}_{\mathrm{x}}$ has improved also due to significant advances in the measurements of the precursor compounds involved in primary production of $\mathrm{HO}_{\mathrm{x}}$ which are in addition to ozone photolysis, mainly photolysis of nitrous acid (HONO), formaldehyde (HCHO), and other oxygenated VOC like acetone. Speciated peroxyradical measurements $\left(\mathrm{HO}_{2}\right.$ and $\left.\mathrm{RO}_{2}\right)$ have been made possible by CIMS coupled to chemical amplification (see below). Instruments capable of measuring the total $\mathrm{OH}$ reactivity have been developed using LIF $\mathrm{OH}$ instruments and allow an estimation of the contribution of still unknown compounds and reactions with $\mathrm{OH}$.

Night-time chemistry is very much driven by $\mathrm{NO}_{3}$ radicals. During the past decade new, compact, mobile, and highly sensitive optical absorption instruments for $\mathrm{NO}_{3}$ have been developed which enable measurements on all platforms and consequently in all parts of the atmosphere. These methods use highly reflective optical mirror cavities to achieve sub-ppt sensitivity for $\mathrm{NO}_{3}$. They can also be used to indirectly detect $\mathrm{N}_{2} \mathrm{O}_{5}$ which forms in a chemical equilibrium together with $\mathrm{NO}_{3}$ and $\mathrm{NO}_{2}$. The availability of the cavity based instruments and their ability to measure both key players simultaneously induced a revival of the scientific interest in nighttime chemistry of oxygenated nitrogen compounds.

To test the current understanding of chemical transformation and removal of primarily emitted gaseous compounds, a number of 
studies have investigated the changing pattern of primary and secondary trace gases in the plumes of cities and biomass burning by probing the plumes at various chemical ages using instruments mounted on mobile platforms like aircrafts and ships (see Monks et al., in this issue). This has been made possible by advances in highly time-resolved measurements of organic trace gases comprising NMHC, OVOCs, halogenated organics and alkyl nitrates, and nitrogen oxides species. The techniques behind that are multiple and comprise PTR-MS, new GC approaches, and new optical methods.

\subsubsection{Mass spectrometric analytical techniques}

The principle of mass spectrometry using ionisation methods at atmospheric pressure (API) has enormously increased the analytical power of mass spectrometry. In particular the atmospheric pressure chemical ionisation (APCI) and the electrospray ionisation (ESI) methods are now widely used in routine analysis (see also section on PTR-MS and $\mathrm{HO}_{\mathrm{x}}$ radicals below). ESI and APCI are highly efficient for polar compounds in either the positive or negative ion mode. For non-polar compounds atmospheric pressure photo ionisation (APPI) provides an alternative method (Syage et al., 2000). These techniques are based on the ionisation with a single UV photon with a wavelength of $\lambda=123.8 \mathrm{~nm}(10 \mathrm{eV})$. Atmospheric pressure laser ionisation (APLI) (Constapel et al., 2005) is the youngest technique among the variety of ionisation methods operating at ambient pressure. APLI uses resonant enhanced mulitphoton ionisation (REMPI).

\subsubsection{Cavity supported optical absorption spectroscopy - \\ CRDS and CEAS}

An overview of cavity supported absorption spectroscopic techniques for atmospheric trace gases is given by Ball and Jones (2003) and Brown et al. (2003). Cavity ring down spectroscopy (CRDS) was developed by O'Keefe (Scherer et al., 1997) and is a highly sensitive form of laser absorption spectroscopy that in recent years has proved useful for measurements of stable compounds as well as radicals and aerosol extinction in ambient atmosphere and laboratory experiments. The centrepiece of the CRDS technique is a high quality (reflectivity of the mirrors $>99.99 \%$ ), two-mirror stable resonator into which pulsed laser light is injected through one end mirror. This mirror reflects most of the incident light but a small fraction couples into the cavity and decays ('rings down') exponentially in time, with the time decay - in the empty cavity - mainly determined by the mirror losses. When an absorbing compound is introduced into the cavity and the laser is tuned to an absorption resonance, the losses rise and the ring down time is reduced. From the reduction in the time constant, the number density of the absorbing species can be determined directly. This detection principle has the big advantage of being insensitive to intensity fluctuations of the light source. The zero ring down signal is achieved by measurement of the sample gas without the absorbing species, e.g. for $\mathrm{NO}_{3}$ radical measurements after chemical titration of $\mathrm{NO}_{3}$ with $\mathrm{NO}$ added into the detection cell (chemical modulation technique). The technique needs no in-field calibration. With state-of-the-art mirrors and laser sources absorption signals in the $10^{-11} \mathrm{~cm}^{-1}$ range can be detected with $1 \mathrm{~s}$ time resolution. Examples of its applications in ambient measurements are exemplified below; for applications in kinetic studies, see Section 3.3.1.

Cavity enhanced absorption spectroscopy (CEAS) is a technique related to CRDS and is often employed with continuous wave light sources. In CEAS, a constant flow of light entering through the cavity's input mirror replenishes the light lost due to reflection losses and absorption by the sample. It has been shown that the light circulating within the cavity reaches steady state within a few ring down times and that its steady state intensity is directly proportional to the ring down time. Consequently, the sample's absorbance per unit path can be calculated from measurements of the steady state intensity with and without the absorbent present. CEAS provides absolute absorption measurements only if the reflectivity of the cavity's mirrors is known as a function of wavelength across the bandwidth of the measurement. The mirror reflectivity is either measured directly via conventional CRDS or deduced from a CEAS calibration spectrum of a known amount of an absorber with known cross-section. A variety of light sources has been used so far for CEAS, from coherent lasers, to incoherent Xe-discharge lamps and light emitting diodes (LEDs). The requirements to the intensity stability of the light source are more demanding than in CRDS.

\subsubsection{Use of isotopes for characterization of gas-phase compounds}

In recent years, major advances in isotopic studies of gas-phase species mostly relate to water isotope measurements from aircraft and satellites to isotopes in methane and non-methane hydrocarbons and their oxidation products formaldehyde ( $\mathrm{HCHO}), \mathrm{H}_{2}$ and CO. For further details, the reader is referred to Monks et al. (in this issue). Measurements of stable isotope ratios are by now an established tool to gain detailed insight into processes determining sources and sinks of important atmospheric trace gases such as carbon dioxide, carbon monoxide, methane, hydrogen, nitrous oxide and ozone. Of specific interest to this section is the recent development of VOC isotopic analysis, which had not begun until about a decade ago (Rudolph et al., 1997) and it took several years before more detailed results from applications of this method became available. Two recent reviews have covered this young, rapidly expanding field (Goldstein and Shaw, 2003; Rudolph, 2007). Isotope information is believed to provide important further insight into VOC sources and sinks. The advantage of the isotope approach over other methods is that it involves measurement of two or more isotopologues of the same species, which eliminates possible bias from comparing different species with different sources, atmospheric life times and sinks. Atmospheric measurements are mostly restricted to ${ }^{13} \mathrm{C} /{ }^{12} \mathrm{C}$ ratios, but measurements of ${ }^{2} \mathrm{H} /{ }^{1} \mathrm{H}$ in compounds other than methane and possibly ${ }^{37} \mathrm{Cl} /{ }^{35} \mathrm{Cl}$ (for example, in methyl chloride, $\mathrm{CH}_{3} \mathrm{Cl}$ ) are on the horizon.

Measurements are performed with gas chromatograph isotope ratio mass spectrometry (GC-IRMS). Upon emission, VOCs contain characteristic isotope ratios. Due to slightly different chemical reaction rate constants of molecules with different isotope composition, the source isotope ratio is altered when the compounds undergo chemical reactions in the atmosphere. Carbon kinetic isotope effects for the reactions of VOCs with $\mathrm{OH}$ radicals and $\mathrm{O}_{3}$ have been established in laboratory experiments. The interpretation of the stable isotope ratios of VOCs is based on the Isotopic Hydrocarbon Clock concept, which allows the estimation of the average photochemical age of VOCs in an air mass (Rudolph and Czuba, 2000). Moreover, the stable isotope ratios of reactive compounds can be measured, such as isoprene and its oxidation products, and the corresponding kinetic isotope effects for the interpretation of atmospheric measurements are also available.

\subsection{Compound specific in situ measurements}

\subsubsection{Atmospheric radicals}

3.2.1.1. $\mathrm{NO}_{3}$ radicals $-\mathrm{N}_{2} \mathrm{O}_{5}$. In the late 1970s the measurement of $\mathrm{NO}_{3}$ radicals in the troposphere was pioneered by Platt and Perner (1980) (Platt et al., 1980). They used long-path differential optical absorption spectroscopy (DOAS) which since then has been a standard technique for atmospheric $\mathrm{NO}_{3}$ detection. DOAS is widely used in different setups in field experiments (e.g. Platt et al., 1981; Allan et al., 2000; Stutz et al., 2004; McLaren et al., 2004; Sommariva et al., 2007; Vrekoussis et al., 2004, 2007) and chamber studies (e.g. Wängberg et al., 1997; Bossmeyer et al., 2006). In the 1980ies matrix isolation electron spin resonance (MI-ESR) was developed 
and applied for $\mathrm{NO}_{3}$ measurements (Mihelcic et al., 1993). However, this technique suffered from its slow time resolution (30 min) and enormous handling effort. Within the last years new complementary techniques have been developed and deployed to field campaigns which render possible highly sensitive atmospheric $\mathrm{NO}_{3}$ measurements with excellent time resolution on all platforms including ships, aircraft, towers and simulation chambers.

Three new techniques for $\mathrm{NO}_{3}$ detection have been developed: Cavity ring down spectroscopy (CRDS), Cavity enhanced absorption spectroscopy (CEAS) (see section above for those 2 techniques) and laser-induced fluorescence (LIF). More detailed descriptions of the usability, sensitivity, and selectivity of cavity ring down spectroscopy and cavity enhanced and laser-induced fluorescence for detection of atmospheric $\mathrm{NO}_{3}$ radicals can be found in recent publications by Simpson (2003); Ball and Jones (2003), Brown et al. (2001, 2002a,b), Ayers et al. (2005), Bitter et al. (2005), Dubé et al. (2006), Wood et al. (2003) and Matsumoto et al. (2005). Many of these techniques have been extended to indirectly measure $\mathrm{N}_{2} \mathrm{O}_{5}$ simultaneously with ambient $\mathrm{NO}_{3}$ in a second channel. Here an inlet line heated up to $90-100{ }^{\circ} \mathrm{C}$ is used wherein $\mathrm{N}_{2} \mathrm{O}_{5}$ decomposes into $\mathrm{NO}_{2}$ and $\mathrm{NO}_{3}$ and the $\mathrm{NO}_{3}$ formed is detected.

LIF is a well established accurate, sensitive, and highly selective method for measurement of atmospheric trace species and free radicals. A pulsed laser is used to excite $\mathrm{NO}_{3}$ radicals to an excited electronic state, followed by measurement of the subsequent fluorescence emission. Selectivity is achieved by tuning the excitation wavelength to a specific absorption transition of $\mathrm{NO}_{3}$. Matsumoto et al. (2005) use excitation of the $627 \mathrm{~nm}$ absorption band, Wood et al. (2003) tune the laser to the strongest absorption line centred at $662 \mathrm{~nm}$. In both instruments $\mathrm{N}_{2} \mathrm{O}_{5}$ can be measured using a second heated inlet line where $\mathrm{N}_{2} \mathrm{O}_{5}$ decomposes and the $\mathrm{NO}_{3}$ formed is detected. These instruments need careful calibration.

3.2.1.2. $\mathrm{OH}, \mathrm{HO}_{2}, \mathrm{RO}_{2}$ and total reactivity. Since the beginning of the 1990 s, measurements of the $\mathrm{OH}$ radical have greatly improved in sensitivity and time resolution by breakthroughs in the analytical technique of LIF and CIMS. Still, problems exist in calibration and artefact suppression, thus DOAS remains the reference method with no calibration necessary, however, with limited time resolution and detection limit of $100 \mathrm{~s}$ and $1 \times 10^{6} \mathrm{molec} \mathrm{cm}^{-3}$, respectively. Employing the new OH-techniques (LIF and CIMS) during campaigns and on mobile platforms like ships and aircraft has substantially improved our knowledge of $\mathrm{OH}$ chemistry but also indicated gaps in our understanding. In clean environments, models are generally able to reproduce the measured $\mathrm{HO}_{\mathrm{x}}$ concentrations within the uncertainties. However, in areas strongly influenced by biogenic emissions, measured $\mathrm{OH}$ is significantly higher than modelled $\mathrm{OH}$ (DiCarlo et al., 2004). Also in high $\mathrm{NO}_{\mathrm{x}}$ environments, models mostly underestimate $\mathrm{HO}_{\mathrm{x}}$ measurements. At Hohenpeissenberg, a rural and forested site in Central Europe, more than $90 \%$ of the $\mathrm{OH}$ variance could be attributed to $\mathrm{J}\left(\mathrm{O}^{1} \mathrm{D}\right)$ and only marginal impact of chemistry on $\mathrm{OH}$ variance was observed. These results were obtained in a long-term study of more than 5 years by CIMS (Rohrer and Berresheim, 2006).

Peroxyradicals have been measured for many years by PERCA (Peroxy Radical by Chemical Amplification) and MI-ESR (Mihelcic et al., 1978). Since the mid $1990 \mathrm{~s} \mathrm{HO}_{2}$ has been successfully measured by LIF as OH after titration of $\mathrm{HO}_{2}$ by $\mathrm{NO}$ (Brune et al., 1995; Mather et al., 1997). Recently, CIMS-OH instruments have been adopted for measurements of $\mathrm{RO}_{2}$ and $\mathrm{HO}_{2}$ by amplified conversion to $\mathrm{H}_{2} \mathrm{SO}_{4}$ via $\mathrm{OH}$ in a chain reaction with $\mathrm{NO}$ and $\mathrm{SO}_{2}$ added, and using controlled ratios of $\mathrm{NO} / \mathrm{O}_{2}$, e.g. the ROXMAS $\left(\mathrm{RO}_{\mathrm{x}}\right.$ Chemical Conversion/CIMS, Hanke et al., 2002) and PERCIMS methods (PEroxy Radical Chemical ionisation Mass Spectrometer, Edwards et al.,
2003). At low $\mathrm{NO} / \mathrm{O}_{2}$ this technique measures the sum of $\mathrm{RO}_{2}$ and $\mathrm{HO}_{2}: \mathrm{RO}_{2}$ reacts with $\mathrm{NO}$ to form $\mathrm{RO}, \mathrm{RO}$ yields $\mathrm{HO}_{2}$ by reaction with $\mathrm{O}_{2}$, and $\mathrm{HO}_{2}$ reacts with $\mathrm{NO}$ to yield $\mathrm{OH}$ which is then titrated by $\mathrm{SO}_{2}$ and detected in the mass spectrometer as sulphuric acid. At high ratios of $\mathrm{NO} / \mathrm{O}_{2}$, however, $\mathrm{RO}$ reacts with $\mathrm{NO}$ to form RONO, and only the initially existing $\mathrm{HO}_{2}$ contributes to the chain reaction yielding sulphuric acid. The LIF $\mathrm{HO}_{2}$-technique and the PERCIMS technique have been successfully intercompared by Ren et al. (2003).

Progress in the quantification of the total amount of reactive pollutants has been achieved by the development of instruments that measure the total atmospheric $\mathrm{OH}$ loss rate (Kovacs and Brune, 2001; Sadanaga et al., 2004; Sinha et al., 2008; Hofzumahaus et al., 2009). These techniques have been used successfully to assess the budget of measured $\mathrm{OH}$ in field campaigns and to quantify missing $\mathrm{OH}$ reactivities in forested and urban regions (e.g. DiCarlo et al., 2004; Sadanaga et al., 2005) as well as in the free troposphere (Mao et al., 2008). Current approaches generally use photolytically produced $\mathrm{OH}$ radicals, a reaction system in which ambient air is allowed to react with $\mathrm{OH}$ for a controlled time interval, and detection of the remaining $\mathrm{OH}$ radicals by LIF. Two technical concepts have been followed so far: the flow-reactor technique (Kovacs and Brune, 2001) and the laser flash and probe method (Sadanaga et al., 2004). In the flow-reactor technique, ambient air is pulled through a flow tube and in the centre of the tube, $\mathrm{OH}$ is injected from an axially moveable $\mathrm{OH}$ source (photolysis of water vapour), and allowed to react in the air flow until the $\mathrm{OH}$ is sampled by a nozzle of the LIF instrument. By moving the $\mathrm{OH}$ injector in and against flow direction, different reaction times can be adjusted. A first-order decay-rate can be fitted to the data which, after subtraction of wall losses, corresponds to the total OH-reactivity. In the laser flash and probe method, ambient air is pumped through a flow-tube cell and $\mathrm{OH}$ is generated by a laser flash applied axially through the centre of the cell. The laser photolyses $\mathrm{O}_{3}$ which forms $\mathrm{OH}$ radicals after reaction of $\mathrm{O}\left({ }^{1} \mathrm{D}\right)$ with water, and the subsequent decay of the $\mathrm{OH}$ is monitored with high time resolution by a LIF instrument sampling from the centre of the flow tube. As in the other technique, the first-order decay is fitted to the data and the wall and diffusion losses are subtracted using the first-order decay obtained from zero-air measurements. Due to uncertainties in the technique, especially the accurate determination of the $\mathrm{OH}$ losses in zero-air, these instruments can measure $\mathrm{OH}$ reactivities above approximately $1 \mathrm{~s}^{-1}$, which is appropriate for most conditions and even covers pristine environments.

3.2.1.3. $\mathrm{OH}$ and $\mathrm{HO}_{2}$ inter-comparisons. Environmental chambers have been essential to provide reliable inter-comparison studies of key atmospheric compounds. For example, within the quality assurance activities of the EU-ACCENT project, an $\mathrm{OH}$ and $\mathrm{HO}_{2}$ inter-comparison was conducted in the atmosphere simulation chamber SAPHIR at Jülich in 2005 (Schlosser et al., 2009). For the first time, the major techniques applied for $\mathrm{HO}_{\mathrm{x}}$ radical measurements were assembled including differential optical absorption spectroscopy (DOAS), laser-induced fluorescence (LIF), chemical ionisation mass spectrometry (CIMS) and matrix isolation electron spin resonance (MI-ESR) with instruments from Germany, England and Japan. As an example, a scatter plot of the results obtained under such controlled conditions by two independent $\mathrm{OH}$ measurement techniques is shown. Excellent agreement between LIF and DOAS was observed and the scatter of the data around the regression line could be explained by the precision of the measurements (Fig. 11, Schlosser et al., 2007).

\subsubsection{VOC measurements}

3.2.2.1. VOC measurements by gas chromatography. New in situ GC systems for operation at remote stations have been developed 


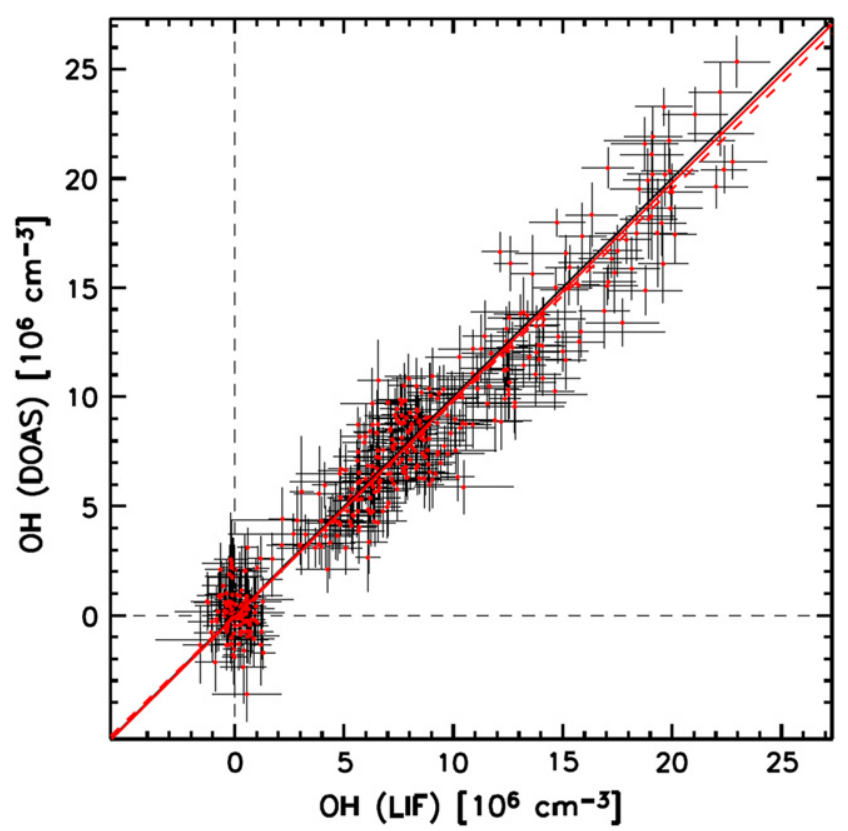

Fig. 11. Scatter plot of $\mathrm{OH}$ concentrations (dots with $1-\sigma$ error bars) measured using the Jülich DOAS and LIF instruments in the SAPHIR chamber of Research Centre Jülich, Germany. From Schlosser et al. (2007).

with substantial improvements in sensitivity (down to below ppt), precision (low and sub \%-range), unattended and cryogen-free operation and enhanced number of different compounds measured in a single analysis. Some of the systems are optimized for robustness and low-power consumption in unattended and cryogen-free monitoring of VOC at remote, unmanned sites (e.g. Tanner et al., 2006) others with no electrical power restrictions are often optimized for certain compound classes. For example, systems based on GC-MS (and GC-FID) have been developed for OVOC analysis (e.g. Hopkins et al., 2003a,b; Legreid et al., 2007). But also comprehensive systems designed to analyse all relevant organic species in one system have been developed, basically multi-channel systems with multiple columns and mostly FID, ECD and MS detectors. Such systems are capable of analysing up to about 100 species comprising hydrocarbons, OVOCs, alkyl nitrates, sulphur containing gases, and halogenated compounds with detection limits in the low ppt range and precision ranging from 0.3 to $15 \%$ (Sive et al., 2005; Goldan et al., 2004). With focus on halogenated compounds, AGAGE has developed the cryogen-free system "MEDUSA" and deployed the system in a global network to achieve 12 measurements per day with precisions of up to $0.1 \%$ (Miller et al., 2008). This MEDUSA system is currently used in the development of a number of new GC systems to accomplish measurements not only of the halogenated compounds, but also the NMHC, biogenic and oxygenated compounds. However, with all these promising developments it should be kept in mind that these instruments are highly sophisticated and a number of severe analytical problems have to be considered, associated with pre-concentration on traps, interaction of analytes with surfaces (adsorption, blanks), water removal, trapping material and artefact production by $\mathrm{O}_{3}$ (Koppmann et al., 1995; Helmig, 1997; Komenda et al., 2003). Thus it is essential to test these systems in intercomparison experiments (see below).

Fast gas chromatography systems capable of measuring with cycle times of some 5 min have been developed which are needed for the use on mobile platforms like aircraft. They offer the required time resolution and the chance to measure OVOCs which are hard to measure in canisters or from cartridges due to their polarity and associated potential sampling artefacts (Apel et al., 2003b; Singh et al., 2004). Comprehensive GC (GC $\times$ GC) techniques offering an unprecedented separation power for a huge number of different organic compounds, have been used in several systems to analyse complex ambient air composition (e.g. Lewis et al., 2000; Xu et al., 2003; Bartenbach et al., 2007). However, this technique is demanding, requires a high amount of liquid nitrogen and manpower, and compared to GC-MS does not offer substantially more compound speciation. Thus, it is rarely used in ambient VOC analysis in atmospheric chemistry.

Atmospheric measurement of the highly reactive and low-volatility mono- and sesquiterpenes is still a challenge mainly due to low concentrations, adsorptive and chemical losses and artefact formation. However, owing to their high importance for atmospheric chemistry, e.g. OH-reactivity, improved methods are needed. PTRMS does not help to overcome these problems since it does not separate between isomers. Generally, methods still comprise ozone removal, adsorptive sampling, thermo-desorption and GC-FID/MS. Problems can, however, be reduced when measurements of emission rates from plant enclosure techniques are applied, since then concentrations are higher and oxidants can be suppressed by appropriate enclosure gas pre-treatment (Ortega et al., 2007, 2008, Ortega and Helmig 2008).

3.2.2.2. Further methods for the detection of atmospheric OVOCs. OVOCs are measured by various methods with considerable recent advances. Formaldehyde can be analyzed by both remote (DOAS, FTIR) and in situ instruments (fluorometric Hantzsch, chromatographic DNPH, PTR-MS, TDLAS, new optical methods and GC). Inter-comparisons between the methods show a mixed picture with disagreement still occurring depending on the meteorological conditions (Hak et al., 2005; Steinbacher et al., 2004; Still et al., 2006; Wisthaler et al., 2008). Recent developments include the optimization of the fluorimetric Hantzsch instrument for flight campaigns (Junkermann and Burger, 2006) and the adaptation of PTR-MS (see section on PTR-MS). Furthermore, a gas chromatographic method with pulsed discharge helium ionisation detection (PD-HID) has been proposed and tested in a field campaign (Hopkins et al., 2003a,b), however, the method shows some $\mathrm{O}_{3}$ and humidity artefacts. In addition, the detection of $\mathrm{HCHO}$ by laser absorption spectroscopy has advanced fast. Thus, the laborious tuneable diode laser systems (TDLAS) (e.g. Kormann et al., 2002) will probably be gradually replaced by the more sophisticated quantum cascade laser spectrometers. An example of the feasibility of this technique has been given by Herndon et al. (2007), measuring formaldehyde (and formic acid) by a pulsed quantum cascade laser spectrometer during an aircraft campaign over the US.

For the remaining OVOCs the method which has been most frequently used in the past was based on sampling of the analytes on DNPH impregnated resins with subsequent derivatization and analysis by liquid chromatography. Although this method is only suitable for analysis of aldehydes and ketones it is still widely used in networks (e.g. EMEP). Apart from the gas chromatographic methods described before, the ground-breaking new development was the introduction of the proton-transfer reaction mass spectrometer (PTR-MS).

3.2.2.3. VOC and OVOCs by proton-transfer-reaction-mass spectrometry (PTR-MS). Proton-transfer-reaction mass spectrometry (PTR-MS) is a technique that allows real-time measurements of volatile organic compounds (VOCs) in air with a high sensitivity and a fast time response (Lindinger et al., 1998a,b). Air to be analyzed is continuously pumped through a drift tube reactor, and a fraction of the VOCs is ionized in proton-transfer reactions with hydronium ions $\left(\mathrm{H}_{3} \mathrm{O}^{+}\right)$and measured by a quadrupole mass spectrometer. Numerous VOCs of atmospheric interest can be monitored with a high sensitivity (10-100 pptv) and rapid response 
time (1-10 s). The technique does not require any sample treatment such as drying or pre-concentration. A major disadvantage is that PTR-MS only determines the mass of product ions, which is not a unique indicator of the VOC identity. It is clear that isomers cannot be distinguished, and the interpretation of mass spectra is further complicated by the formation of cluster ions and the fragmentation of product ions. de Gouw and Warneke (2007) give an overview on published inter-comparisons between PTR-MS and alternative methods performed in urban air, forest air, or the free troposphere. Slopes of the scatter plots (PTR-MS versus alternative method) were found to be equal to 1 within $20 \%$ for the compounds or compound groups methanol, acetonitrile, propylene, acetaldehyde, acetone, isoprene, methyl vinyl ketone + methacrolein, methyl ethyl ketone, PAN, benzene, toluene, styrene, $C_{8}$-aromatics, $\mathrm{C}_{9}$-aromatics. Deviations were found for dimethyl sulfide for which an interference from $\mathrm{H}^{+}\left(\mathrm{CH}_{3} \mathrm{CHO}\right)\left(\mathrm{H}_{2} \mathrm{O}\right)$ clusters occurs. In some studies the PTR-MS measurement of monoterpenes (protonated $\mathrm{m} / \mathrm{z}$ 137) compared fairly well with the sum of multiple species while one study showed rather poor agreement. The identifications of the $m / z$ signals are often only tentative: they are based on the variability of the signal in time, space and with altitude, and on the covariance with other measurements. Therefore, some identification could be very specific for certain air masses, and have a very limited applicability to other regions of the atmosphere.

Because $\mathrm{HCHO}$ has a proton affinity only slightly higher than that of water, backward reaction of protonated $\mathrm{HCHO}$ with water significantly reduces the instrument's sensitivity to HCHO and also leads to a humidity dependence of the measurement. A poor correlation with independent measurements of $\mathrm{HCHO}$ by the Hantzsch fluorescence technique was found by Steinbacher et al. (2004), suggesting that fragments of other compounds could interfere with the signal at $m / z$ 31. An inter-comparison of 5 analyzers at a simulation chamber showed a relatively good agreement of the PTR-MS with other techniques, but the PTR-MS was operated in an optimized mode of operation which however may not be optimal for the measurement of other species (Wisthaler et al., 2008). A detection limit of 0.15-0.25 ppb was reached for $\mathrm{HCHO}$. Inomata et al. (2008) found that correction of the signal for humidity and possible fragments of methyl hydroperoxide, methanol and ethanol led to better agreement with MAXDOAS measurements under ambient conditions.

The big advantages of PTR-MS, fast measurement and low detection limit, were exploited to perform flux measurements of a wider suite of volatile organic compounds by the eddy covariance (EC) technique (Brunner et al., 2007; Spirig et al., 2005; Karl et al., 2000, 2001a, 2002, 2004; Schade and Custer, 2004; Holzinger et al., 2006; Davison et al., 2008). Since for multi-component EC measurements detection of each compound occurs sequentially, special evaluation schemes have been used for the flux calculation, mainly the "virtual disjunct" approach (Karl et al., 2002) and a low-pass filter approach with an averaging over the unresolved high frequencies (Brunner et al., 2007; Spirig et al., 2005). Biogenic emission fluxes of several compounds like methanol, acetone, acetaldehyde, 2-methyl-3buten-2-ol, hexenals, hexanol, hexenols, hexenylacetate, terpenes, and isoprene were measured at forest sites, from agricultural land, grassland, deserts and from hay harvesting.

Several attempts have been undertaken to overcome the identification problem of PTR-MS. By coupling a gas chromatographic column to a PTR-MS instrument (Karl et al., 2001b; de Gouw et al., 2003; Davison et al., 2008) contributions from different VOCs to a single mass channel can be separated. However, this method results in the loss of the real-time measuring capabilities of the instrument if applied continuously. Another method used to resolve isobars is replacing the quadrupole mass spectrometer by an ion trap mass spectrometer which allows differentiating isomers based on their different fragmentation patterns (Warneke et al., 2005a,b). High resolution time-of-flight mass spectrometry was developed to resolve nominally isobaric species (Blake et al., 2004; Graus et al., 2007). Compound mixes such as acetone/glyoxal, isoprene/furan or naphthalene/octanal can thus be distinguished.

3.2.2.4. VOC inter-comparisons. The international NMHC intercomparison exercises NOMHICE (Apel et al., 1999, 2003a) and AMOHA (Plass-Dülmer et al., 2006) greatly improved and documented the quality of laboratories performing ambient VOC measurements. For the first time, during AMOHA also an initial step was introduced allowing for each participant to sample real ambient air simultaneously with an on-line system at Hohenpeissenberg, Germany. Results of this blind comparison documented the ability of most laboratories to perform ambient VOC analysis at continental clean air sites (Plass-Dülmer et al., 2006). Within GAW, the World Calibration Centre (IMK-IFU, Garmisch-Partenkirchen, Germany) conducted a series of station audits and comparisons and, thus, greatly helped to improve the quality and comparability of VOC measurements (Rappenglück et al., 2006). During an ACCENT-OVOC inter-comparison in the atmospheric simulation chamber SAPHIR in Jülich, Germany, (see Figs. 12 and 13) various state of the art instruments for the measurements of formaldehyde and/or OVOCs were studied in 2005. Formaldehyde measured by methods based on DOAS, Hantzsch fluorimetry, DNPH cartridges and HPLC, and a new optimized mode of PTR-MS achieved fair agreement between the methods, individual problems could by identified and improvements are expected (Wisthaler et al., 2008). Similarly, OVOCs were anonymously intercompared between different methods based on PTR-MS, GC-FID, GC-MS, DNPH cartridges and HPLC, catalytic conversion followed by Hantzsch fluorimetry for methanol, and DOAS for selected compounds. Although several problems with individual methods and instruments were identified, all methods yielded very good agreement with the reference concentrations for the majority of the selected compounds and conditions. Thus, all methods proved to be generally suitable for OVOC measurements (Apel et al., 2008). However, further tests involving lower concentrations and a test gas matrix more close to ambient conditions will be needed in the future.

\subsubsection{Nitrogen species}

Nitrous acid has reliably been measured for a long time only by DOAS (differential optical absorption spectroscopy) (Perner and Platt, 1979). Other techniques often suffer from potentially substantial analytical problems due to interferences and (lightinduced) chemical artefacts at surfaces (Kleffmann et al., 2006). Several techniques transfer the gaseous HONO into a sampling solution and either detect the nitrite by ion chromatography or by light absorption after forming an azo dye from chemical reaction in the sampling solution (for recent reviews, see Clemitshaw, 2004; Kleffmann, 2007). Due to the potential for artefacts, however, it is essential to demonstrate the feasibility against other techniques, preferably DOAS (Kleffmann et al., 2006; Acker et al., 2006). The recently developed LOPAP technique (LOng Path liquid Absorption Photometer, Heland et al., 2001) combines high sensitivity (1 ppt) with high time resolution (4 min) (Kleffmann et al., 2006). It has been shown to agree with DOAS down to the detection limit of DOAS of about 200 ppt within the uncertainties, however, it should be noted that typical daytime concentrations of HONO are mostly below 200 ppt. Thus, there is the need to further verify wet techniques by other reference methods in the future, which mainly considers new optical methods like cavity ring down spectroscopy. LOPAP has been applied in a series of recent field- as well as laboratory studies which suggest that HONO formation in the atmosphere may have a larger impact on atmospheric chemistry 


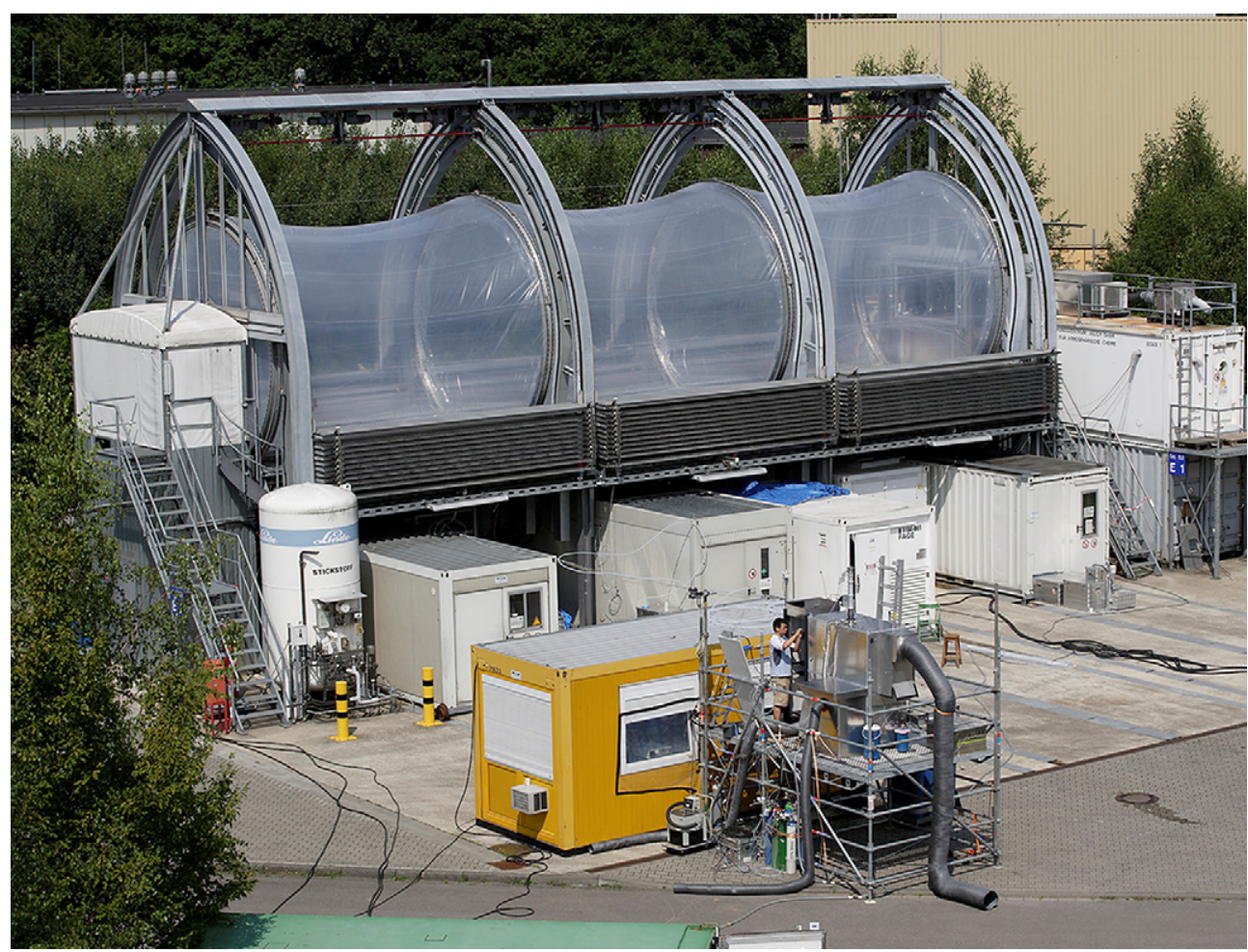

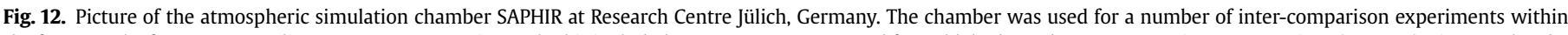

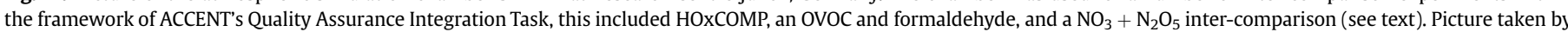
Hans-Peter Dorn, Research Centre Jülich.

than previously thought, due to photo-enhanced formation of HONO on organic (humic substances) as well as on inorganic surfaces (see discussion of $\mathrm{NO}_{\mathrm{y}}$ chemistry, in Monks et al., in this issue). A modified version of the method, where $\mathrm{HNO}_{3}$ is reduced to HONO, allows determination of the sum of $\mathrm{HNO}_{3}$ and $\mathrm{N}_{2} \mathrm{O}_{5}$ in the atmosphere (Kleffmann et al., 2007). Work is currently in progress to develop a modified LOPAP instrument which could be used to simultaneously measure $\mathrm{N}_{2} \mathrm{O}_{5}$ and $\mathrm{HNO}_{3}$. It is important to mention the use of TDLAS and QC-TDL (both mentioned earlier) to measure HONO and $\mathrm{NH}_{3}$, possibly the best techniques available for these species (Clemitshaw, 2004; Li et al., 2008, 2006).

Recently, a photo-acoustic sensor for analysis of ammonia in ambient air with a sensitivity of $0.1 \mathrm{ppb}$ has been developed and become commercially (TGA310, Omnisens SA, Switzerland) available (Schilt et al., 2004). The basic improvement in this system compared to other photo-acoustic systems is the suppression of interferences by using two channels for sampling, and the same

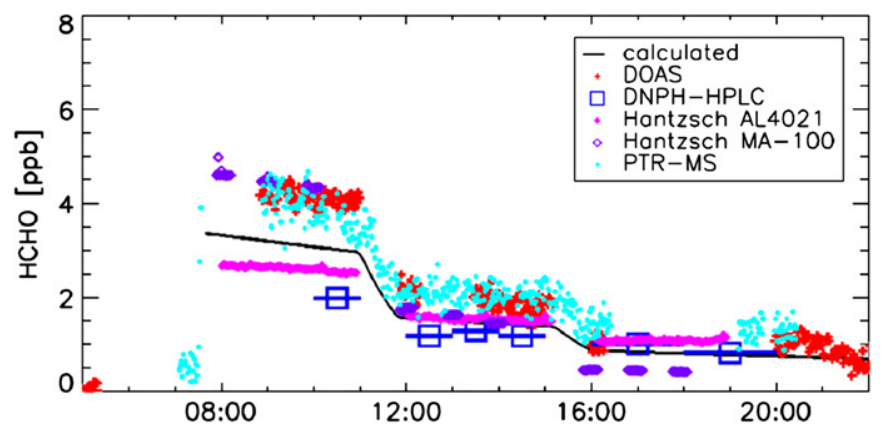

Fig. 13. Time series of measured and calculated $\mathrm{HCHO}$ mixing ratios during the zeroair experiment with humidity and ozone added (dewpoints at -3 to $-7{ }^{\circ} \mathrm{C}$, ozone at 40-50 ppb and temperature close to $0^{\circ} \mathrm{C}$ ). Adapted from Wisthaler et al. (2008). photo-acoustic cell for detection. In a background channel, ambient air is drawn over a citric acid coated diffusion denuder to selectively remove $\mathrm{NH}_{3}$ with no effect on the interfering compounds water vapour, ethene, carbon dioxide, and $\mathrm{NH}_{3}$ from the thermal dissociation of particulate ammonium nitrate. The instrument switches between both channels and the difference signal is used to determine ammonia.

Additionally, very sensitive optical absorption techniques are becoming available, especially cavity ring down spectroscopy (CRDS) with detection limits approaching the range of $0.1 \mathrm{ppb}$.

A new method for $\mathrm{NO}_{\mathrm{y}}$ components has been reported by Day et al. (2002) named "thermal dissociation laser-induced fluorescence" (TD-LIF). This method offers fast and sensitive analysis of various organic and non-organic fractions of $\mathrm{NO}_{\mathrm{y}}$.

Table 3 summarizes all techniques discussed in the present section.

\subsubsection{Carbon monoxide}

Carbon monoxide measurements have long time been based on GC methods with catalyst and FID or mercury detector. In the late 1990s the already existing method of vacuum-UV resonance fluorescence was optimized (Gerbig et al., 1999), and a commercially available system has been developed comprising excellent sensitivity, time resolution, and precision in an instrument which does not need much maintenance. Thus, it is well suited to operation on mobile platforms and at remote stations for continuous monitoring.

\subsection{Instrumental developments for gas-phase kinetics}

The study of gas kinetics related to atmospheric chemistry is a relatively mature field of science where a variety of well-established experimental techniques are available and a considerable database of high quality measurements of kinetic data has been 
Table 3

Recently developed new measurement techniques and achieved detection limits (DL) and time resolution (time) (abbreviations: PTRMS: proton-transfer reaction mass spectrometry, ROXMAS: $\mathrm{RO}_{\mathrm{x}}$ chemical conversion chemical ionisation mass spectrometry, SI-CIMS: selected ion chemical ionisation mass spectrometry, CRDS: cavity ring down spectrometry, CEAS: cavity enhanced absorption spectrometry, LIF: laser-induced fluorescence, LOPAP: long path liquid absorption photometer).

\begin{tabular}{|c|c|c|c|}
\hline Technique & Compounds & DL/time & Reference \\
\hline PTR-MS & $\begin{array}{l}\text { Hydrocarbons, PAN, } \\
\text { carbonyls, } \\
\text { acetonitrile } \mathrm{HCHO}\end{array}$ & $\begin{array}{l}10-100 \text { pptv, } 1-10 \mathrm{~s} \\
0.15-0.25 \mathrm{ppb}, 2 \mathrm{~s}\end{array}$ & $\begin{array}{l}\text { See text } \\
\text { Wisthaler et al., } 2008\end{array}$ \\
\hline ROXMAS & $\mathrm{HO}_{2}, \mathrm{RO}_{2}$ & & Hanke et al., 2002 \\
\hline SI-CIMS & $\begin{array}{l}\mathrm{HNO}_{3} \\
\mathrm{HO}_{2} \mathrm{NO}_{2} \\
\mathrm{SO}_{2} \\
\text { PAN } \\
\text { PPN }\end{array}$ & $\begin{array}{l}5-50 \text { pptv, } 1-10 \mathrm{~s} \\
3-10 \text { pptv, } 1-30 \mathrm{~s} \\
5-20 \text { pptv, } 1-30 \mathrm{~s} \\
10 \text { pptv, } 1 \mathrm{~s} \\
10 \text { pptv, } 1 \mathrm{~s}\end{array}$ & Huey and Tanner, 2006 \\
\hline CRDS & $\begin{array}{l}\mathrm{NO}_{3} / \mathrm{N}_{2} \mathrm{O}_{5} \\
\mathrm{OIO} \\
\mathrm{I}_{2}\end{array}$ & $\begin{array}{l}1 \mathrm{pptv} \text { in } 100 \mathrm{~s} \\
4 \mathrm{pptv} \text { in } 10 \mathrm{~min} \\
20 \text { pptv in } 20 \mathrm{~min}\end{array}$ & $\begin{array}{l}\text { Brown et al., } 2001 \\
\text { Ball and Jones, } 2003\end{array}$ \\
\hline CEAS & $\mathrm{NO}_{3} / \mathrm{N}_{2} \mathrm{O}_{5}$ & & Ball et al., 2004 \\
\hline LIF & $\begin{array}{l}\mathrm{NO}_{3} / \mathrm{N}_{2} \mathrm{O}_{5} \\
\text { Lifetime } \mathrm{OH} \\
\mathrm{HCHO}\end{array}$ & $\begin{array}{l}1 \mathrm{~s}^{-1}, 3.5 \mathrm{~min} \\
20 \mathrm{pptv}, 1 \mathrm{~min}\end{array}$ & $\begin{array}{l}\text { Wood et al., } 2003 \\
\text { Mao et al., } 2008 \\
\text { Tan, } 2006\end{array}$ \\
\hline LOPAP & $\begin{array}{l}\mathrm{HONO} \\
\mathrm{HNO}_{3}\end{array}$ & $\begin{array}{l}1 \mathrm{pptv}, 4 \mathrm{~min} \\
5-30 \mathrm{pptv}, 6-2 \mathrm{~min}\end{array}$ & $\begin{array}{l}\text { Kleffmann et al., } 2006 \\
\text { Kleffmann et al., } 2007\end{array}$ \\
\hline
\end{tabular}

created. However, only a relatively small fraction of the reactions of relevance to atmospheric chemistry has been studied experimentally, and many aspects of atmospheric gas phase chemistry still need to be further investigated. Furthermore, the incorporation of new techniques has led to exciting developments in the laboratory. In most cases these new techniques have also found important applications in field studies (e.g. PTR-MS and CRDS).

\subsubsection{Gas-phase kinetics}

Many laboratory studies of atmospheric gas phase chemistry aim at extending the database on kinetics and product formation by reactions between volatile organic species and radicals, such as $\mathrm{OH}$, $\mathrm{NO}_{3}$ or halogen atoms, or ozone. Typically, new results have allowed information to be gained not only on the reactions of larger and more complex (sometimes multifunctional) molecules than those studied previously, but also on reactions where previous studies need to be confirmed, discrepancies need to be clarified or temperature dependencies need to be determined. There are a large number of examples of such studies, e.g. several studies on unsaturated oxygenated compounds. Some compounds have been studied particularly well because they are (or are candidates for) being produced in large quantities for industrial applications and thus their likely environmental fate needs to be understood (e.g. CFC replacements, Sellevag et al., 2004, and hydrazoic acid, Orlando et al., 2005).

The progress in experimental determination of kinetic data for atmospheric chemistry applications is reflected in the compilations of evaluated kinetic and photochemical data for atmospheric chemistry, e.g. that of the IUPAC Subcommittee for Gas Kinetic Data Evaluation (http://www.iupac-kinetic.ch.cam.ac.uk/, also published on ACP, e.g. Atkinson et al., 2008). A useful new development regarding such compilations is the creation of a comprehensive database on the reactions of oxygenated VOCs at the CNRS in Orleans, France, http://www.era-orleans.org/eradb/.

Most kinetic and mechanistic studies of gas phase atmospheric chemistry are carried out based on techniques that have been commonly applied for some decades and have proved to be very versatile. For chamber studies, on-line FTIR spectroscopy is particularly important as a very useful means of measuring reactants and products in a mixture. However, the incorporation of novel mass spectrometric techniques such as PTR-MS has extended both the range of compounds that can be detected along with the sensitivity. Particularly notable is the use of TOF-PTRMS in the study of aromatics oxidation by Wyche et al. (2009). Also other forms of CIMS have frequently found applications in laboratory studies. A particularly interesting new result was obtained by applying a chemical ionisation mass spectrometer coupled with a high pressure turbulent flow reactor to the gas phase reaction between $\mathrm{NO}$ and $\mathrm{HO}_{2}$ radicals, which was found to have a minor channel forming $\mathrm{HNO}_{3}$ (Butkovskaya et al., 2005).

In flow tube systems, laser flash-photolysis is frequently used, combined with various detections systems, for the detection of both radicals and stable molecules. A recent variation on the traditional flash-photolysis technique involves the use of a CCD camera to record a spectrum over several wavelengths simultaneously. Stone and Rowley (2005) successfully used this technique to detect $\mathrm{HO}_{2}$ radicals and to measure the rate of their self reaction.

Cavity Ring Down Spectroscopy (see Section 3.2.1.2) has also played a large role in recent laboratory studies. Its high sensitivity has been used for the measurement of both weak absorptions of unstable molecules (e.g. Stark et al. (2008), $\mathrm{HO}_{2} \mathrm{NO}_{2}$ overtone spectra) and the spectra and kinetics of free radicals. Among the examples of such radical measurements are the studies by Krasnoperov et al. (2005) of formyl radical reactions, by Rajakumar et al. (2008) of quantum yields for the photolytic formation of acetyl radicals and by Pope et al. (2005) of the formation of formyl radicals by the photolysis of formaldehyde. An interesting variant of the technique has been developed by Fittschen and co-workers to monitor $\mathrm{HO}_{2}$ in the near-IR (Thiebaud et al., 2007). More established techniques such as laser-induced fluorescence continue to be used, and have led to substantial revisions in rate coefficients previously measured by less sensitive techniques (Sivakumaran and Crowley, $2003 \mathrm{OH}+\mathrm{CH}_{3} \mathrm{CHO}$; Karunanandan et al., $2007 \mathrm{OH}+$ hydroxy acetaldehyde; Dillon et al., $2006 \mathrm{OH}+$ hydroxyacetone). The technique has also been used to obtain absolute rate coefficients for alkoxy radical reactions (Devolder, 2003).

Studies of temperature dependencies are essential for applications of kinetic data to tropospheric chemistry, considering the wide range of temperatures that may be encountered in the troposphere. If a reaction can proceed along more than one pathway, the branching ratios between such pathways may often depend on temperature. A recent review (Seakins and Robertson, 2007) discusses new developments in methods for the observation of product branching ratios in gas phase reactions. It is particularly important to understand how these branching ratios depend on temperature, especially if there is a change in the relative amounts of radical versus stable products, since this can affect the rate of ozone production. Recent examples of laboratory studies of temperature dependencies of branching rates are the studies by Cassanelli et al. (2007) of the formation of some organic nitrates from the reaction between NO and peroxyradicals, the study by Orlando (2007) of the oxidation of diethyl ether and the study by Albu et al. (2006) on the reaction of OH with DMS. Kinetic studies at very low temperatures have been made possible by the use of Laval nozzle expansion, a recent development is the use of pulsed Laval nozzle expansions (e.g. used by Spangenberg et al., 2004 for studies of OH-radical reactions at temperatures between 58 and $300 \mathrm{~K}$ ).

A great deal of laboratory research on gas phase chemistry in recent years has been focused on reactions that lead to formation of products with low volatility with a capacity to condense on existing particles, or even to contribute to the formation of new particles. Information on such reactions has been obtained by chemical analysis and physical characterization of these particles, as 
discussed in Section 1. Reactions that appear to be particularly relevant for the formation of secondary organic aerosol (SOA) are those of terpenes (mono- as well as sesquiterpenes), of isoprene and also of aromatic hydrocarbons, however atmospheric SOA formation may also be influenced by a number of other precursors, including some that currently are not accounted for (Hallquist et al., 2009). The efforts to clarify the often complex chemistry involved in the formation of condensable products from SOA precursors has motivated the development of improved sampling and analysis procedures for volatile oxidised organic species (OVOCs) in the gas-phase, both on-line (particularly by mass spectrometric and spectroscopic techniques as discussed above) and in methods for sampling with subsequent off-line analysis (normally by chromatographic techniques). An example of the latter is the use of Solid Phase Microextraction, where sampling is done on a fibre coated with an extracting phase (e.g. Holt et al., 2005). Also heterogeneous reactions, where volatile gaseous species are taken up by and react on particles, or where gaseous species are released from particles, have been studied extensively in recent years, as discussed in Section 2.1.1. Along with the progress made by experimental efforts to determine SOA properties in the laboratory, the framework for describing phase partitioning in models has also been improved. A recent important development has been the introduction of the volatility basis set approach (Donahue et al., 2006). For a thorough review of recent experimental studies regarding the process involved in the formation of SOA as well as a discussion of the current and emerging research issues, we refer to the review by Hallquist et al. (2009).

\subsubsection{Synergies with theoretical studies}

While theoretical studies of gas-phase kinetics have benefited from the large amount of high quality experimental data that has been obtained in the last decades, the experimentalists have benefited from the refinement of theory and the development of software tools to perform $a b$ initio calculations which allow evaluations of the likelihood of reaction pathways and provide estimates of rate coefficients. These tools are now accessible to all workers in the fields of environmental science and laboratory studies of gas kinetics are nowadays very frequently accompanied by theoretical calculations. Recent progress in the theoretical understanding and modelling of gas-phase kinetics has mainly been in electronic structure theory, and thus in the calculation of potential energy surfaces, and in methods for simulating the complex temperature and pressure dependence of rate coefficients (Seakins and Robertson, 2007, and references herein). A recent example of a study based on a combination of experimental and theoretical work is the investigation of the atmospheric oxidation mechanism of isoprene carried out by Fan and Zhang (2004).

\subsubsection{Structure activity relationships}

Measuring all rate coefficients with relevance to atmospheric chemistry is clearly an impossible task; the existence of more than 7000 reactions represented in the Master Chemical Mechanism gives an idea of what would be required (http://www.chem.leeds.ac.uk/ Atmospheric/MCM/main.html). However, structure-activity relationships (SARs) have been developed based on the existing experimental results with the aim of predicting unknown rate coefficients. SARs may provide a correlation between rate coefficients and structural characteristics of a molecule or some of its measured or calculated physical properties (e.g. ionisation potential). While such relationships work for simple, mono-substituted compounds, it has become clear that they are less easy to apply to multifunctional compounds, especially where electronegative groups are involved. Le Calve et al. (1997a,b) have shown that "long-range" interactions govern the reactivity further than the nearest neighbour interactions imply. Furthermore, the recognition that hydrogen-bonded complexes can influence reactivity challenges the idea that reactions can be represented in terms of simple bond additivity.

The rate coefficient data obtained over the last few years has considerably helped to improve the group substituent factors which are used in estimations of rate coefficients using the group additivity SAR method. This is particularly true in the case of oxygenated VOCs. However, uncertainties still remain and knowledge of temperature dependency for many of the substituent factors is still required. The use of topological and geometric descriptions of molecules in structure-activity relationships (SARs) has recently been shown to be a powerful technique; strong correlations have been observed for the reactions of various classes of NMHC with free radicals (McGillen et al., 2006a,b). The SARs based on topological indices compare favourably with other literature methods.

\subsection{Aircraft-based observation of atmospheric gases}

Intensive aircraft missions provide important data and can help in the validation of satellites, but they are not useful for identifying anomalies, inter-annual variabilities and long-term trends. Routine aircraft missions such as MOZAIC (Marenco et al., 1998; Cammas et al., 2009, http://mozaic.aero.obs-mip.fr/web/) and CARIBIC (Brenninkmeijer et al., 2007, http://www.caribic-atmospheric.com/) are providing extremely valuable data sets of a number of key species with sufficient measurement frequency that are much more useful to this end. A non-exhaustive list of recent studies that could not have been achieved without routine aircraft measurements of ozone, water vapour, and carbon monoxide includes: i) process studies, like the effect of mixing on the atmospheric composition of the mid-latitude tropopause layer (Brioude et al., 2008) or the formation of meridional ozone gradients in the African upper troposphere (Sauvage et al., 2007), ii) the evidence of regional and seasonal anomalies, like the channelling of pollution by persistent atmospheric pressure centres over the Eastern Mediterranean sea (Kalabokas et al., 2007), or the documentation of the vertical extension of pollution throughout the continental boundary layer during the European heat wave in 2003 (Tressol et al., 2008), iii) the evaluation of the ozone trends in the troposphere over the subtropics (Bortz et al., 2006), the mid-latitudes (Thouret et al., 2006; Zbinden et al., 2006) and over China (Ding et al., 2008).

\subsection{Remote sensing of gaseous composition from the ground}

There exist many different remote-sensing techniques for atmospheric gases. Some are passive methods, like optical spectrometric techniques using natural sources of light, other ones are active techniques, like the LIDAR, in which the instrument emits light into the atmosphere and records the return signal that is altered through its interaction with the atmosphere.

Hereinafter, we will focus on two passive optical spectrometric techniques for remote sensing of the gaseous composition of the atmosphere, that have evolved quite significantly in recent years. As discussed in more detail below, the Fourier-transform infrared (FTIR) spectrometry and the Differential Optical Absorption Spectroscopy (DOAS) technique, now extended to Multi-axis DOAS or MAX-DOAS technique, have not only improved regarding the precision and accuracy of the observed data, but they have also extended the information content of the data from total absorber column amounts to vertical profile information.

\subsubsection{Fourier-transform infrared spectrometry}

Ground-based measurements of solar absorption spectra in the infrared provide information about the concentrations of the absorbing atmospheric molecules. Indeed, many atmospheric species have several absorption features in the infrared 
corresponding to specific vibration-rotation transitions. High spectral resolution enables minimization of interferences between different lines as well as the resolution of some information concerning the vertical distribution of the target absorber. A good overview of remote sensing of the atmosphere in the infrared and its history can be found in Brown et al. (1992). The most up-to-date technique to record the high resolution solar absorption infrared spectra is Fourier-transform infrared (FTIR) spectrometry. It has the advantage that a large spectral range can be observed simultaneously, in a short time (of the order of minutes), while maintaining a high spectral resolution (spectral resolving power $\sim 10^{6}$ ) and a high signal-to-noise ratio $\left(\sim 10^{3}\right)$. The disadvantage is that the technique requires stable, clear-sky conditions. In the absence of sun during polar winter or night, the FTIR observation can be made using the moon as the light source (Notholt and Lehmann, 2003). A full moon is required (and clear sky) to have a high enough signalto-noise ratio. Moreover the lunar absorption spectra include a contribution from the earth atmosphere emission, making the interpretation of the spectra more difficult. Absorption spectroscopy in the UV and visible can also be used for remote sensing of atmospheric composition from the ground, the ozone measurements by the Dobson instrument being the best known example. As measurements can be done using scattered light, they are not limited to clear sky situations and are easily automated. Recent developments combining measurements taken under different viewing directions (MAX-DOAS) greatly enhanced the applicability of this technique to tropospheric composition measurements.

Another option to make measurements of the atmospheric composition in the absence of sunlight is to use atmospheric emission features.

Atmospheric observations by solar absorption FTIR spectrometry started in the 1980s. Since then, the technique has been implemented at about 25 ground-based stations in the frame of the Network for the Detection of Atmospheric Composition Change (NDACC; http://www.ndacc.org). The measurements are performed mostly in the mid infrared $\left(\sim 600-4500 \mathrm{~cm}^{-1}\right)$. In this spectral range, one can detect a large number of tropospheric as well as stratospheric species, including ozone (Schneider et al., 2008a), chlorinated and fluorinated source and reservoir species, nitrogenated species including $\mathrm{HNO}_{3}$ and $\mathrm{NO}_{2}$, sulphur compounds like OCS, pollutants like $\mathrm{CO}$ and $\mathrm{HCHO}$, and greenhouse gases like $\mathrm{H}_{2} \mathrm{O}$, $\mathrm{CO}_{2}, \mathrm{~N}_{2} \mathrm{O}, \mathrm{SF}_{6}$, as well as organic compounds and biomass burning products like $\mathrm{HCN}, \mathrm{C}_{2} \mathrm{H}_{6}, \mathrm{C}_{2} \mathrm{H}_{2}, \mathrm{C}_{2} \mathrm{H}_{4}$, etc. In many cases, one can also distinguish the different isotopologues of the species, for example, the different isotopologues of water vapour (Schneider et al., $2006 b$ ), or ozone (Meier and Notholt, 1996). In addition, work is in progress to detect species like ${ }^{12} \mathrm{CO}$ and ${ }^{13} \mathrm{CO},{ }^{13} \mathrm{CH}_{4}$ and $\mathrm{CH}^{3} \mathrm{D}$. Substantial work has been done in recent year to improve the FTIR measurements (Schneider and Hase, 2008) and intercompare with other techniques (Schneider et al., 2005, 2008b).

As water vapour is a strong absorber in the mid-infrared, one finds almost always absorption lines that interfere with the target lines. Therefore, the implementation of FTIR observations at humid sites, e.g. in the tropics at low altitude, is more challenging. Nevertheless, it has been demonstrated recently that many of the key atmospheric species can be detected also at low altitude, tropical stations (Petersen et al., 2008; Senten et al., 2008).

The most direct products of the FTIR absorption observations are the total column abundances. The total column abundance of an absorber is directly proportional to the area under the observed target absorption line, as long as this one is not saturated. In the last decade, algorithms have been developed to retrieve - in addition to the total column abundance - information about the vertical distribution of the absorber in the atmosphere, based on the dependence of the absorption lineshape on the local pressure and temperature. In the mid-infrared, absorption linewidths are typically of the order of $0.05-0.1 \mathrm{~cm}^{-1}$ at the surface (pressure-broadened width) decreasing to $\leq 0.005 \mathrm{~cm}^{-1}$ in the stratosphere (Doppler-broadened width). Therefore the vertical profile retrieval, especially in the stratosphere, really requires high spectral resolution measurements. Above the altitude where the pressure-broadening reduces to the Doppler width, no more vertical information can be retrieved.

The vertical resolution of the retrieved data is limited to the order of a few kilometres. For example, for ozone, one achieves a vertical resolution of the order of 5-8 $\mathrm{km}$, between ground and about $35 \mathrm{~km}$ altitude (Barret et al., 2002; Fig. 14). For several species that have an important abundance in the troposphere like $\mathrm{CH}_{4}$, one can distinguish the tropospheric column abundance from the stratospheric one. This evolution towards the retrieval of vertical profile information has increased the use of ground-based FTIR data for satellite validation (e.g. Hartogh and Espy, 2006; Richter and Wagner, 2008) and for trend analysis in different 'layers' (altitude ranges) of the atmosphere (e.g. Vigouroux et al., 2008). For several tropospheric species that are observed by current satellite experiments, like $\mathrm{CO}, \mathrm{CH}_{4}$ and $\mathrm{N}_{2} \mathrm{O}$, ground-based solar absorption FTIR spectrometry is the only technique that provides data that are easily comparable to the satellite products (contrary to the in situ surface network data) (e.g. Barret et al., 2003; Sussmann et al., 2005; Sussmann and Buchwitz, 2005; Dils et al., 2006; Velazco et al., 2005; Notholt et al., 2003).

Recently, it has been demonstrated that FTIR solar absorption spectrometry is one of the techniques that provides the most precise estimates of the total column of water vapour in the atmosphere (Schneider et al., in press; Schneider and Haze, 2009), and that has the capability of measuring total ozone with a precision around 1 DU under favourable conditions (Schneider and Hase, 2008).

\subsubsection{Trace gas profile measurements using \\ the MAX-DOAS technique}

Multi-axis differential optical absorption spectroscopy (MAXDOAS) is a newly introduced passive remote-sensing technique allowing for automated monitoring of the vertical distribution of a number of tropospheric and stratospheric trace gases. Building upon the concept of differential absorption spectroscopy (see Platt and Stutz, 2006 for a review on DOAS) developed a decade ago for tropospheric measurements using active systems as well as for stratospheric trace gas monitoring within the Network for the Detection of Atmospheric Composition Change (NDACC, www. ndsc.ncep.noaa.gov), the MAX-DOAS technique relies on observations under different elevation angles, which allows for better sensitivity to trace gases close to the instrument. For an instrument operated at the ground, this means high sensitivity to trace gases in the boundary layer and the possibility of retrieving up to 4 independent pieces of information about the vertical distribution in the lower troposphere (Hönninger et al., 2004; Wittrock et al., 2004). However, as for all passive remote-sensing techniques using UV-visible solar radiation as a source, measurements are limited to daytime and require clear-sky conditions for best accuracy.

MAX-DOAS observations have been applied to automated monitoring of tropospheric $\mathrm{NO}_{2}$ (e.g. Hönninger et al., 2004; Leigh et al., 2006, 2007), HCHO (Heckel et al., 2005), glyoxal (Sinreich et al., 2007), BrO (Frieß et al., 2004; Theys et al., 2007; Fietkau et al., 2007), IO (Frieß et al., 2001) as well as aerosol extinction profiles (Wittrock et al., 2004; Irie et al., 2008). Most of these studies however are still exploratory in nature, aiming at the scientific demonstration of a new technique under development.

In addition to their profiling ability, MAX-DOAS instruments also provide accurate measurements of the tropospheric column 


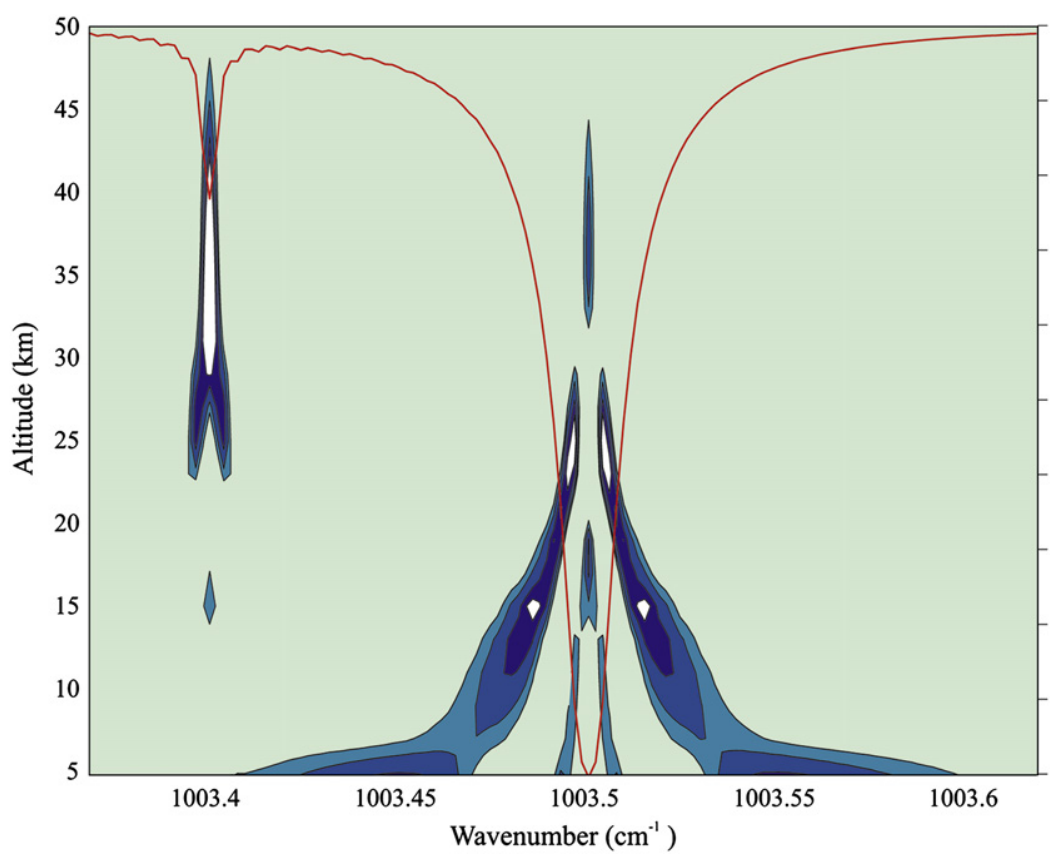

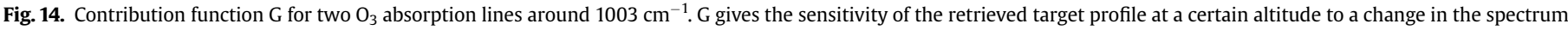

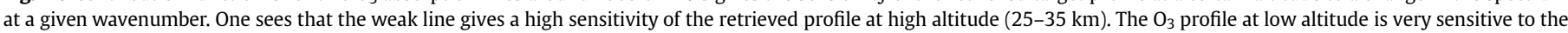
wings of the strong almost saturated line. From Barret (2003).

amounts of trace gases. Column measurements are largely independent of variations in boundary layer height which is an advantage for the determination of total atmospheric load as e.g. needed for emission studies. MAX-DOAS derived tropospheric columns are also well suited for validation of satellite measurements of tropospheric species (e.g. Brinksma et al., 2008).

\subsection{Observation of tropospheric trace gas from space}

\subsubsection{Past and current space-borne sensors}

The remote sensing of atmospheric constituents and surface parameters from space builds on the activities of the research community and space agencies. Atmospheric observations began with the first satellites some 50 years ago at the beginning of the space age. The NASA Nimbus programme, which ran from 1963 to 1993, was a pioneering programme in Earth observations. A very successful series of satellites was constructed and flown, which made unique contributions to the remote sensing of the atmosphere and Earth observation. In addition a variety of pathfinder and other NASA missions contributed significantly. The Soviet programme was also active in the development of atmospheric remote sensing. It initiated the long-term measurements of many important atmospheric parameters including the measurements of the vertical profile of ozone and aerosols. The Nimbus experiments contributed significantly to establishing the discovery of the ozone hole. The UARS mission followed and led to the Mission to Planet Earth, which resulted in a series of platforms targeting the remote sounding of the atmosphere and the Earth's surface. The majority of the atmospheric remote sounding satellites were initially aimed at measuring the composition of the upper atmosphere.

Early observations of tropospheric species (CO) from space came from MAPS measurements on board the space shuttle but only for very limited periods of time. The first extended data set of satelliteobserved tropospheric composition came from the TOMS instrument (Krueger and Jaross, 1999), an ultraviolet-visible instrument which was designed to observe total ozone columns but which can also provide, in combination with external information or assumptions, tropospheric ozone columns, at least in the tropics. The Global Ozone Monitoring Experiment GOME (Burrows et al., 1999) was the first satellite instrument having enough spectral resolution to observe not only ozone but also some of its precursors $\left(\mathrm{NO}_{2}, \mathrm{HCHO}\right)$ and also $\mathrm{BrO}$ which is involved in catalytic destruction of ozone in the polar boundary layer in spring time. These measurements are continued and extended by the SCIAMACHY instrument (Bovensmann et al., 1999) which has better spatial resolution and NIR observing capabilities, facilitating $\mathrm{CO}, \mathrm{CH}_{4}$, and $\mathrm{CO}_{2}$ observations. Starting from 1996, space-borne instruments using the infrared spectral range to sound the atmosphere complemented the aforementioned missions and allowed systematic observations of key infrared absorbing molecules. Tropospheric species such as $\mathrm{CO}, \mathrm{CO}_{2}$, methane, ozone and nitric acid $\left(\mathrm{HNO}_{3}\right)$ were measured by IMG/Adeos (Kobayashi et al., 1999), the first Fourier-Transform spectrometer dedicated to tropospheric remote sensing. In the same spectral range, using gas correlation techniques, the MOPITT/Terra instrument (Drummond and Mand, 1996) has been observing $\mathrm{CO}$ in the upper and middle troposphere since its launch at the end of 1999. Along with AIRS/AQUA (launched in 2002, Aumann and Pagano, 1994), TES/Aura (launched in 2004, Beer et al., 2001) and more recently IASI/METOP (Schlussel et al., 2005), these 4 instruments routinely provide trace gases distributions for a series of molecules. Using higher spectral resolution in the limb geometry, vertical profiles as well as a number of other tropospheric species including several hydrocarbons can also be observed, such as with MIPAS/ENVISAT (limb sounding, Fischer et al., 2008) and ACE (solar occultation, Bernath et al., 2005) satellite instruments.

After the initial focus on the upper atmosphere, there are now several instruments dedicated to atmospheric chemistry that are routinely providing data with unprecedented resolution and sampling. Depending on their primary goals, these current sensors use different spectral ranges, and instrumental specifications. The 

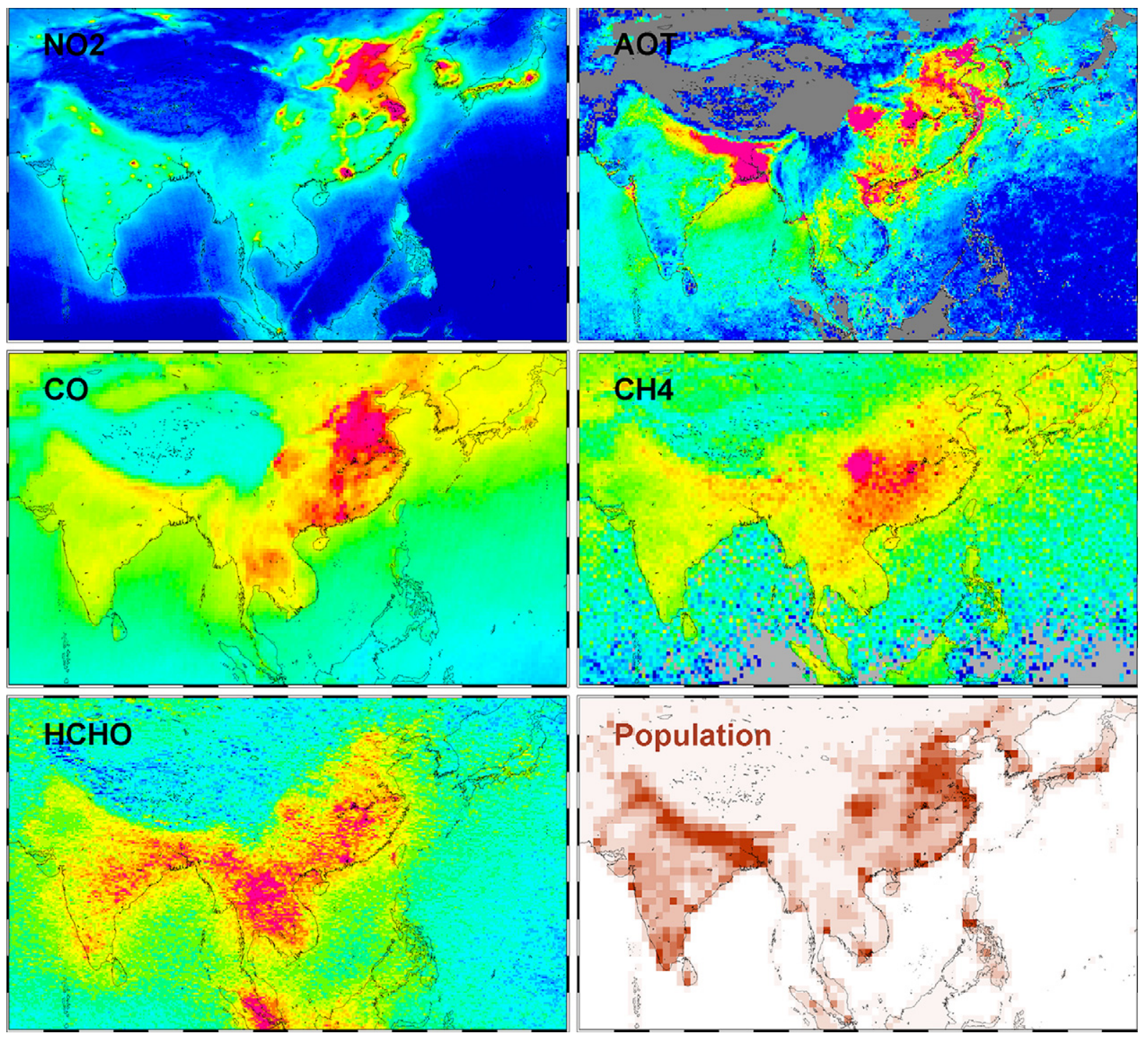

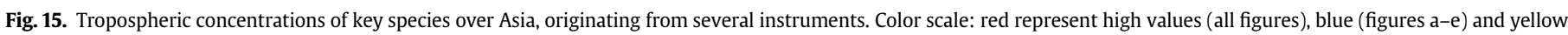

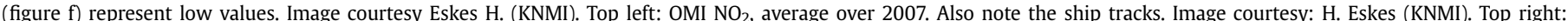

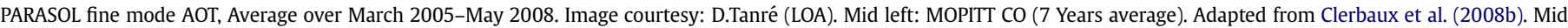

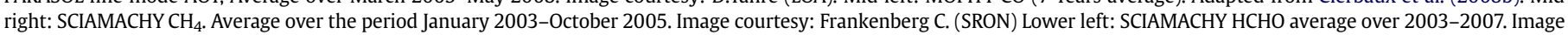
courtesy: DeSmedt I. (BIRA) Lower right: Population number. Source: CIESIN, in million inhabitants.

concentrations of trace species are retrieved from the atmospheric spectrum recorded, using the characteristics of the absorptionemission features in the dedicated spectral regions. The space missions deliver total column or vertical profiles for a variety of reactive molecules, with a variable accuracy, horizontal sampling and vertical resolution.

Fig. 15 illustrates the capabilities of currently flying instruments to provide tropospheric trace gas distributions all over the globe, with an example over Asia. It is a composite plot of measurements obtained by a range of instruments using different spectral ranges and sounding techniques (see caption). In the next sections, examples are given for global measurements of ozone, ozone precursors and other chemically active climate relative trace gases.

\subsubsection{Measurements of gaseous emission from space}

3.6.2.1. Measurement of nitrogen oxides. Nitrogen oxides $\left(\mathrm{NO}_{\mathrm{x}}=\mathrm{NO}+\mathrm{NO}_{2}\right)$ are emitted from all combustion processes making them one of the most important anthropogenic pollutants. Improvements in emission controls and the switch to cleaner fuels has led to significant decreases in $\mathrm{NO}_{\mathrm{x}}$ emissions in many industrialised countries while economic development and increasing use of fossil fuels results in upward trends in $\mathrm{NO}_{\mathrm{x}}$ other parts of the world, most notably in China. While surface networks are available to monitor $\mathrm{NO}_{\mathrm{x}}$ levels in many parts of the world, they do not extend to all relevant regions and do not have the spatial coverage needed to fully assess current changes. With GOME, SCIAMACHY, OMI, and GOME-2, retrieval of tropospheric $\mathrm{NO}_{2}$ column amounts is possible at regional and even better spatial resolution. These data have been used to study the spatial distribution of $\mathrm{NO}_{2}$ and to invert the global distribution of $\mathrm{NO}_{\mathrm{x}}$ emissions and their sources (Jaegle et al., 2005; Martin et al., 2003, 2006; Van der A et al., 2008). Measurements from the GOME instrument have also been used to study ship emissions in remote regions of the oceans where no other measurements are available (Beirle et al., 2004). SCIAMACHY data with their better spatial resolution provide more detailed information over an extended area (Richter et al., 2004), and recently the analysis was further extended to include GOME-2 data (Franke et al., 2008). One particularly interesting application is the 
evaluation of changes in the $\mathrm{NO}_{2}$ fields which can be linked to changes in emissions, a quantity relevant for atmospheric modelling and political decision taking alike. For example, data from the GOME and SCIAMACHY instruments revealed large increases of $\mathrm{NO}_{\mathrm{x}}$ emissions in China over the last decade (Richter et al., 2005; Van der A et al., 2006, 2008) while emissions in Europe and the US decreased at least in some regions.

Owing to the high spatial resolution of the data, emission trends could also be derived at a country level in Europe (Konovalov et al., $2005,2006,2008$ ), the effect of denoxification of power plants in the Eastern US can be studied (Kim et al., 2006) and a reduction of $\mathrm{NO}_{\mathrm{x}}$ emissions in some cities of the Western US can be found. Another approach to study anthropogenic emissions was applied to GOME data by investigating the effect of day of week (Beirle et al., 2003). While similar types of analysis had already been performed on surface measurements, the satellite data provide information on a global scale and highlight interesting differences between the weekly cycle of cities in the US, Europe and China as well as in cities with predominantly Jewish or Muslim inhabitants.

While most of the anthropogenic emissions of $\mathrm{NO}_{\mathrm{x}}$ are emitted close to the surface, lightning produced $\mathrm{NO}_{\mathrm{x}}$ is mainly injected into the upper troposphere where its lifetime is much longer than on the ground. However, current estimates of lightning $\mathrm{NO}_{\mathrm{x}}$ still have large uncertainties. This is mainly due to the sparseness of observational data which mainly comes from dedicated aircraft campaigns. Over the last years, satellite data from UV/vis instruments have been used to fill this gap and to derive information on lightning $\mathrm{NO}_{\mathrm{x}}$, both using indirect methods where all contributions except lightning are determined using models and the remaining part is attributed to lightning (Boersma et al., 2005; Choi et al., 2005; Martin et al., 2007) or directly by analysing individual events of lightning and the $\mathrm{NO}_{2}$ columns associated with them (Beirle et al., 2006). This is illustrated in Fig. 16, where GOME $\mathrm{NO}_{2}$ columns above a large thunderstorm cloud in the Gulf of Mexico are compared with lightning flashes derived from the NLDN network. In this case, lightning took place just before the GOME overpass resulting in large $\mathrm{NO}_{2}$ signals above the electrically active part of the thunderstorm. While these studies show clear evidence for lightning produced $\mathrm{NO}_{\mathrm{x}}$, the values derived are at the lower side of the range of current estimates with important implications for the global ozone budget. A recent study analysing the sensitivity of satellite measurements to $\mathrm{NO}_{\mathrm{x}}$ in clouds gives indication for higher sensitivity to $\mathrm{NO}_{\mathrm{x}}$ than to $\mathrm{NO}_{2}$, which would result in a further reduction of lightning $\mathrm{NO}_{\mathrm{x}}$ estimates from satellite data (Beirle et al., 2009).

3.6.2.2. Sulphur dioxide. Anthropogenic emissions of sulphur dioxide have long been a major pollution problem in industrialised countries. In most of them, $\mathrm{SO}_{2}$ emissions have been greatly reduced over the last decades through sulphur removal from power plant exhausts and the use of sulphur depleted fuels. A notable exception is shipping emissions which continue to have a large $\mathrm{SO}_{2}$ content as a result of lacking regulations. On the contrary, the rapidly increasing use of coal for energy production has led to a strong increase in $\mathrm{SO}_{2}$ emissions in China over the last years leading to severe environmental problems. The current generation of UV instruments has sufficient spectral and spatial resolution to be sensitive to $\mathrm{SO}_{2}$ in the lower troposphere, and satellite measurements of GOME and OMI have been used to study $\mathrm{SO}_{2}$ emissions of smelters and their temporal evolution (Carn et al., 2007). Analysis of the combined time series of GOME and SCIAMACHY shows a similar rapid increase in $\mathrm{SO}_{2}$ over China as was observed for $\mathrm{NO}_{2}$ in broad agreement with bottom up estimates. Improved detection capacities of space-borne sensors have also been used to estimate $\mathrm{SO}_{2}$ emissions from volcanic activity.

In recent years, satellite sounders have increasingly contributed to the monitoring of volcanoes on the global scale, mostly relying, as far as gases are concerned, on the sulphur dioxide signal. The first observations of $\mathrm{SO}_{2}$ were achieved in the UV using selected wavelength pairs of the TOMS instrument (Krueger et al., 1995). With their higher spectral resolution and broader spectral coverage, the GOME, SCIAMACHY, OMI, and GOME-2 instruments provide much better sensitivity, in particular to the lower troposphere (Afe et al., 2004; Eisinger and Burrows, 1998; Yang et al., 2007). Detection sensitivities down to a very few Dobson Units for the $\mathrm{SO}_{2}$ column have been achieved from these instruments, enabling the measurement of both passive degassing and large-scale eruptions. While the primary product of UV retrievals is the integrated $\mathrm{SO}_{2}$ column, the wavelength dependence of the $\mathrm{SO}_{2}$ absorption crosssection facilitates profile retrievals for situations with large $\mathrm{SO}_{2}$ signals. Alternatively, a combination of satellite measurements and trajectory calculations can be used to determine the altitude of volcanic plumes (Eckhardt et al., 2008). In the infrared, $\mathrm{SO}_{2}$ has been successfully measured from large-band sounders, such as MODIS (Watson et al., 2004), ASTER (Pugnaghi et al., 2006; Urai, 2004) or SEVIRI on a geostationary platform (Prata and Kerkmann, 2007). These measurements offer high spatial and temporal sampling and are applicable, as for the UV-visible sounders, for the measurements of passive degassing or eruptive plumes. The low spectral resolution produces, however, large uncertainties in the measured concentrations. The recent launch of sounders with better spectral resolution opens the way for improved sounding capabilities in thermal infrared. First results have been obtained from operation of AIRS (Carn et al., 2005; Eckhardt et al., 2008; Prata and Bernardo, 2007), IASI (Clarisse et al., 2008) and TES (Clerbaux et al., 2009). In the latter two cases, low resolution $\mathrm{SO}_{2}$ profiles have been obtained, adding information on the altitude of the ejection. AIRS and IASI, as operational meteorological sounders offer global coverage twice daily and therefore unprecedented spatial sampling of the plume.
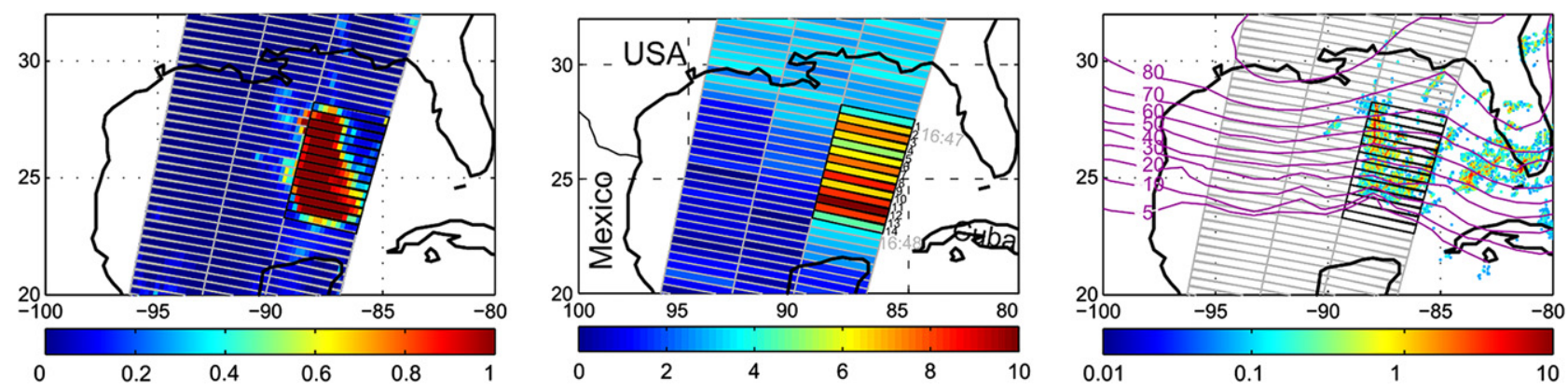

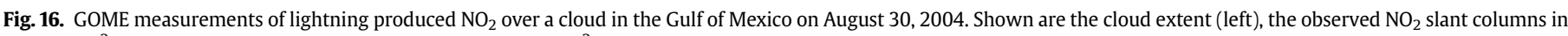
molec $\mathrm{cm}^{-2}$ (middle) and the number of flashes detected per $\mathrm{km}^{2}$ by the NDLN network (right). Adapted from Beirle et al. (2006). 
3.6.2.3. Carbon monoxide and biomass combustion markers. Carbon monoxide ( $\mathrm{CO}$ ) was one of the first tropospheric trace gases observed from space. As result of its relatively long lifetime, it undergoes long-range transport from its sources and mixes both horizontally and vertically. Space-borne observations from MOPITT reveal a hemispheric gradient and high values in the outflow of regions with intense biomass burning (Edwards et al., 2004). Main source regions are fires in Africa and South America but fires in boreal forests in Siberia and Alaska also emit large quantities of CO (Petron et al., 2004; Pfister et al., 2005). As it is dominated by biomass burning emissions, the global $\mathrm{CO}$ burden shows large inter-annual variations following changes in the amount of biomass burning. This indicates that future CO distributions will largely depend on the evolution of anthropogenic and natural fires in response to climate change and farming practice. Although MOPITT measurements have only limited sensitivity in the lower troposphere, the signature of anthropogenic pollution can be observed over industrialised regions in long-term averages (Clerbaux et al., 2008a). The anthropogenic signal can also be retrieved using measurements in the solar IR by SCIAMACHY (Buchwitz et al., 2007; de Laat et al., 2006) as it has full sensitivity down to the ground. However, the signal-to-noise ratio of these measurements is limited, and so far only preliminary assessments of changes in CO from non-fire sources have been derived from satellite data (Shindell et al., 2005).

The fire emission sources can also well be monitored from space using measurements of nitrogen dioxide, formaldehyde or glyoxal in the UV-visible (Wittrock et al., 2006). This is because in the vicinity of the fires, these species are all significantly in excess of their background values. The measurements of $\mathrm{CO}$ can then be used to quantify the fire emission (Pfister et al., 2005; Turquety et al., 2007) and also, given its lifetime of two months, to track the transport and fate of the plumes (Edwards et al., 2006). In addition to these species, observations in the infrared have recently enabled monitoring other, mostly short-lived species. The observations close to fires in nadir geometry include $\mathrm{C}_{2} \mathrm{H}_{4}$, $\mathrm{CH}_{3} \mathrm{OH}, \mathrm{HCOOH}$ and $\mathrm{NH}_{3}$ (Coheur et al., 2009). Measurements from limb sounders in young or aged biomass burning plumes higher up in the free and upper troposphere reveal signatures of small NMHCs $\left(\mathrm{C}_{2} \mathrm{H}_{4}, \mathrm{C}_{2} \mathrm{H}_{6}, \mathrm{C}_{2} \mathrm{H}_{2}\right)$, OVOCs $\left(\mathrm{CH}_{3} \mathrm{OH}, \mathrm{HCOOH}, \mathrm{C}_{3} \mathrm{H}_{6} \mathrm{O}\right)$, $\mathrm{HCN}, \mathrm{NH}_{3}$ and PAN (Clarmann et al., 2007; Coheur et al., 2007; Dufour et al., 2006; Glatthor et al., 2007; Rinsland et al., 2005, 2006, 2007a,b; Steck et al., 2008).

3.6.2.4. Ammonia. Measurements of ammonia in the lowest layers of the atmosphere have been reported for the first time from analyses of the TES satellite data on a local basis (Beer et al., 2008). Very recently, global distributions, highlighting elevated concentrations above fire regions and most importantly above large agricultural valleys in Europe (Fig. 17), Asia and the US, have been gathered from analyses of the IASI satellite measurements (Clarisse et al., 2009). A comparison of these local to global distributions with the TM5 CTM suggests for the agricultural valleys significant underestimates of ammonia emissions in current inventories. These results open the way for reassessing the emission sources of this key tropospheric species, linking gas phase to aerosol chemistry and impacting on the nitrogen budgets and the ecosystems (see Fowler et al., in this issue).

\subsubsection{Space-borne observation of tropospheric halogen oxides}

As in the stratosphere, halogens can efficiently remove ozone in the troposphere if present in higher concentrations. In most parts of the troposphere, halogens are bound in their reservoir substances and reactive halogen compounds are present only in very low concentrations. However, in the polar spring, very large concentrations of $\mathrm{BrO}$ are observed in combination with strongly reduced ozone levels. The full spatial extent of this phenomenon, which is limited to the cold boundary layer of the polar regions in spring, could only be assessed using space-borne observations of the GOME instrument (Wagner and Platt, 1998; Richter et al., 1998). While there are now many more observational data on these so-called $\mathrm{BrO}$ explosions and it is generally assumed that sea salt is the origin of the $\mathrm{Br}$, there still are some uncertainties with respect to the exact mechanisms of initial $\mathrm{Br}$ release. One possible

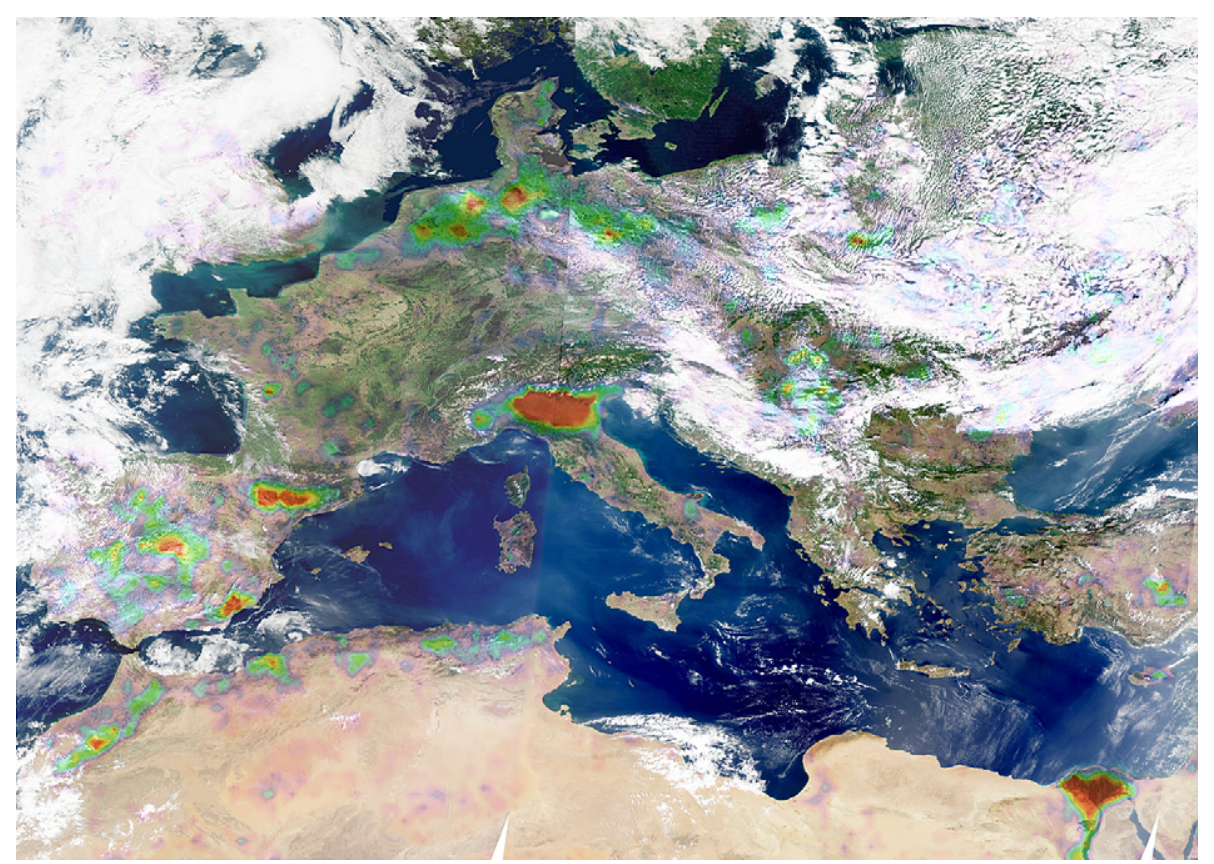

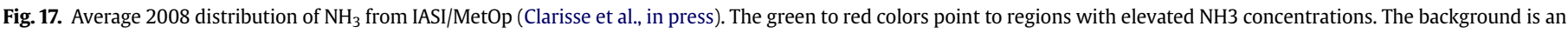
image from MODIS, on August 30, 2008, with the white structures being clouds (credit for MODIS: Gonzalez L. and Deroo C., http://www-loa.univ.lille1fr). 
explanation is the release through frost flowers or aerosol formed from them, and some indication for a role of frost-flowers was found by combining satellite $\mathrm{BrO}$ measurements, sea ice observations and meteorological data (Kaleschke et al., 2004; Jacobi et al., 2006). However, this is not the only possible mechanism (see Simpson et al., 2007b for a review) and further work is needed to fully understand these processes.

In addition to $\mathrm{BrO}$, IO was also identified in the troposphere in some regions with strong biogenic activity. As with bromine, iodine efficiently removes ozone but in addition, higher oxides of iodine can be formed which are involved in new particle formation. In contrast to bromine, the sources of iodine are thought to be to a large part of biological origin. Iodine monoxide columns have recently been retrieved from measurements of the SCIAMACHY instrument (Saiz-Lopez et al., 2007a; Schönhardt et al., 2008) showing clear IO signals over the ice around Antarctica which are in qualitative agreement with ground-based observations (Saiz-Lopez et al., 2007b). There is also an indication of enhanced IO over parts of the Antarctic continent suggesting long-range transport of IO precursors. So far, there is little evidence of comparable high IO amounts in the northern hemisphere.

Ground-based observations have also detected $\mathrm{BrO}$ and $\mathrm{IO}$ in non-polar maritime regions with strong biological activity. So far, these observations could not be reproduced in satellite measurements, probably as a result of the reduced sensitivity to absorptions in the lower troposphere over the dark oceanic surface. Higher spatial resolution of future sensors could facilitate detection of hot-spots even under these conditions. Recent observation of $\mathrm{BrO}$ in the plume of the Kasatochi eruption was demonstrated using measurements of the GOME-2 instrument (Theys et al., 2009) in agreement with previous observations from the ground. The possible measurements of halogen species from high resolution thermal infrared (TIR) sounders (e.g. IASI) is under investigation.

\subsubsection{Space-borne observation of tropospheric ozone}

Ozone is formed in the lower troposphere by complex photochemical reactions involving $\mathrm{NO}_{\mathrm{x}}, \mathrm{CO}, \mathrm{CH}_{4}$ and other VOCs. As is obvious from the above, satellite remote sounders already contribute to the monitoring of tropospheric ozone chemistry and to forecasting air quality by providing quantitative information on these ozone precursors. A significant breakthrough in observing the atmospheric composition from satellites comes from the direct observation of tropospheric ozone itself. Although conceptually straightforward, the practical implementation of this task is challenging as it comes in retrieving a small fraction of ozone from the measured integrated column, which is largely dominated by the stratospheric ozone layer. The UV-visible instruments provide only weak sensitivity to tropospheric ozone while thermal sounders offer maximum sensitivity in this layer, with some vertical profiling ( $\sim 6 \mathrm{~km}$ resolution) capabilities (e.g. Coheur et al., 2005; Nassar et al., 2008; Osterman et al., 2008; Turquety et al., 2002; Worden et al., 2007a). The potential of probing the boundary layer remains, however, limited in most situations.

For tropospheric ozone, a long time series exists from the data of the TOMS instrument in combination with other data sets (Fishman and Brackett, 1997; Ziemke et al., 2001). In the tropics, several different retrieval approaches have been used and increased ozone amounts have been linked to biomass burning and transport from the stratosphere (Martin et al., 2002; Thompson et al., 2000). At midlatitudes, changes in stratospheric ozone amounts complicate the retrieval increasing the uncertainties, and combination of measurements and transport modelling has been applied to derive tropospheric fields from a combination of OMI and MLS measurements (Schoeberl et al., 2007). As UV/visible observations provide tropospheric column amounts or very poor vertical resolution profiles (Liu et al., 2006a), a large part of the observed ozone patterns are linked to tropopause changes and dynamics. However, the signature of pollution and of biomass burning can also be identified. Recent developments in IR satellite instrumentation facilitate the retrieval of vertically resolved ozone in the troposphere albeit usually not down to the surface. Extended analyses have been performed, using TES and AIRS in particular. They have highlighted seasonal trends (Divakarla et al., 2008), enhanced pollution patterns and long-range transport (Jourdain et al., 2007; Parrington et al., 2008; Zhang et al., 2006), while also contributing to climate related issues (Worden et al., 2008). More recently, the enhanced capabilities of TIR sounders to probe tropospheric ozone have been used to perform an analysis of the photochemical pollution events that occurred during the Summer 2007 heat wave in Southern Europe, with the recently launched IASI sounder (Eremenko et al., 2008). Overall this study is a first step in using infrared satellite observations to monitor tropospheric ozone and to improve the forecasts of air quality and climate models.

On the theoretical side, evidence that improvements in measuring tropospheric ozone could be gained by combining information from complementary observations in the UV-visible and the TIR have been obtained (Landgraf and Hasekamp, 2007; Worden et al., 2007b), but the demonstration of application in the field still needs to be made.

\subsubsection{Space-borne observation of methane}

Key climate-related species are very stable gases in the atmosphere, characterized by long life-times. Measurements from satellite are possible using different techniques, but the difficulty is to detect a small variability of the signal over a large spectral signature. In the past two centuries, atmospheric methane has more than doubled and now constitutes $20 \%$ of the anthropogenic climate forcing by greenhouse gases. Yet its sources are not well quantified, introducing uncertainties in its global budget. Global scale observation can provide information that cannot be directly accessed from ground-based observations. Global methane distributions can be retrieved by using space-borne thermal infrared (AIRS, IASI) and near-infrared absorption spectroscopy (SCIAMACHY). In addition to the expected latitudinal North-South gradient, space-borne sensors can detect large-scale patterns of anthropogenic and natural methane emissions. Frankenberg et al. reported from the SCIAMACHY measurements unexpectedly high methane concentrations over tropical rainforests, revealing that emission inventories considerably underestimated methane sources in these regions (Frankenberg et al., 2005, 2008).

Infrared nadir sounders obviously contain information on the major climate gases (e.g. Chahine et al., 2006), whose strong absorption bands in the thermal infrared are the physical reason for the so-called "greenhouse effect". Excluding water vapour, largely variable, these gases are $\mathrm{CO}_{2}, \mathrm{CH}_{4}$ and $\mathrm{N}_{2} \mathrm{O}$. The first global observations of $\mathrm{CH}_{4}$ from AIRS have also been obtained (Xiong et al., 2008), representing mainly the distributions in the free and the upper troposphere. The analyses of the data reveal interesting features in terms of sources, which need further investigations and validations, and some local patterns such as a plume above the Tibetan plateau during the Asian summer monsoon (Xiong et al., 2008). Similar global mapping of $\mathrm{CH}_{4}$ with IASI is possible and a series of related studies is ongoing. The potential to improve on surface measurements from IASI using lines at the shortwave end of the IASI spectra around $2750 \mathrm{~cm}^{-1}$ during daytime is under investigation.

\subsubsection{Characterizing atmospheric transport from space}

3.6.6.1. Boundary layer outflow and free troposphere. Ozone and carbon monoxide are key pollutants which have an average lifetime in the troposphere of the order of several weeks. This makes them 
suitable for monitoring the inter-hemispheric transport of pollution plumes. Because tropospheric ozone retrievals are challenging for the reasons explained above, the CO fields, which are readily available from TIR sounders in the free troposphere, have been primarily used for this purpose. Outflow and inter-continental transport from the northern or southern hemisphere sources has for example been studied with MOPITT (Edwards et al., 2004, 2006). Integrated approaches, combining different satellite and aircraft measurements have added insight into the link between the sources and the observation of pollution in remote regions (Cook et al., 2007; Gros et al., 2004; Heald et al., 2003; McMillan et al., 2008; Stohl et al., 2007a,b; Turquety et al., 2008). It has also been shown that the $\mathrm{O}_{3}-\mathrm{CO}$ correlations give information on the continental outflow of pollution (Zhang et al., 2006) and that satellites could be useful, despite providing only limited vertical sensitivity, for studying the uplift of pollution following deep-convective processes (Jiang et al., 2007; Kar et al., 2004, 2006).

The transport of reactive nitrogen species is another important process for our understanding of global tropospheric composition. Being short-lived, the $\mathrm{NO}_{\mathrm{x}}$ remain mainly close to their emission source. Their chemical transformations lead to $\mathrm{HNO}_{3}$ and PAN which, depending on the local thermodynamic conditions of the atmosphere, can be transported far from the sources. PAN, which is recognized as an important reservoir in the sense it regenerates the $\mathrm{NO}_{\mathrm{x}}$ after thermal decomposition, has not yet been measured from nadir sounding instruments. Total columns of $\mathrm{HNO}_{3}$ can routinely be measured from TIR sounders but, as for ozone, the isolation of its tropospheric part is difficult. A first step in retrieving $\mathrm{HNO}_{3}$ from the high-resolution IMG sounder was recently made (Wespes et al., 2007), suggesting the observation of pollution transport. This opens the way for more extensive distributions to be gathered in the coming years by IASI or AIRS.

As for $\mathrm{NO}_{\mathrm{x}}, \mathrm{SO}_{2}$ is generally quickly removed from the atmosphere by wet deposition. In the case of volcanic eruptions, the $\mathrm{SO}_{2}$ is sometimes emitted higher in the troposphere where it last days to weeks, being then often transported on inter-continental scales, as discussed above.

3.6.6.2. Upper troposphere. Although mainly designed to probe the chemical composition and the processes of the stratosphere, infrared limb-viewing instruments such as MIPAS and the ACE-FTS have in the last years been extremely useful for studying the upper troposphere. These sounders, which enable sounding down to about $6 \mathrm{~km}$ in cloud-free conditions, provide indeed unprecedented sensitivity to the less abundant species due to the long absorption paths. Quasi global distributions have been gathered and analyzed for $\mathrm{C}_{2} \mathrm{H}_{6}, \mathrm{H}_{2} \mathrm{CO}$ and PAN from MIPAS (Glatthor et al., 2007; Steck et al., 2008; Clarmann et al., 2007). In addition, as described above, a vast range of small organic species has been detected locally, mainly in the plumes of young or aged biomass fires. Some of these are now routinely retrieved. From these observations, large-scale features, such as a North-to-South gradient in the concentrations of NMHCs resulting from anthropogenic activities or seasonal variations of some short-lived species have been shown for the first time. Specific processes, relating the observations to biogenic emissions (Dufour et al., 2007) or the vertical outflow of pollution in the Asian monsoon (Park et al., 2008) have been studied. Also the MLS instrument has been used to track CO vertical and horizontal transport (Jiang et al., 2007).

\section{Data assimilation and integration of results}

Observational data will never cover the full spatial and temporal variability of atmospheric composition. Satellite data are often restricted e.g. to cloud free areas, time series are interrupted, discontinued or simply absent at places or times where information is desired, aircraft pass along a single flight track, and lidar data - surfacebased or from space - sample just a curtain. Ideally any such gap can be filled with assimilation techniques, implemented in chemical transport models, and using suitable data with fully characterized errors.

Assimilation attempts to find the best representation of an evolving system when given measurements and prior information on the system, taking into account errors in the measurements and prior information (Lahoz et al., 2007). Assimilation can thus provide 4dimensional fields that can be evaluated against independent observations and applied, e.g. for detailed budget studies (Pradier et al., 2006). Observations often lack a sufficient characterization of the chemical composition and the associated physical parameters. Examples comprise the chemical composition of the aerosol, unresolved in remote sensing products or unmeasurable components of photo-oxidant reaction chains. Assimilation techniques can help by transforming constraints into information about unobserved species. One of the first examples was provided by Elbern et al. (1997) who used observations of only a few species to constrain the ozone and precursors system in air quality applications. Beyond correcting initial fields for observed and non-observed species related through chemistry, assimilation can be used to optimise emission estimates (Elbern et al., 2007), reaction rates and parameters of the chemical system (Menut, 2003), or even winds (Peuch et al., 2000).

Even though considerable progress has been made in assimilating information from various measurement platforms - both, ground- and satellite-based (e.g. the ESA funded PROtocol MOniToring for the GMES Service Element: Atmosphere; http://www. gse-promote.org/ or the European GEMS project), the assessment and attribution of sources and varying source strengths, the regional burden of air pollutants and related impact on air quality and climate remain challenging. The uncertainties in the assimilated fields and our understanding of the numerous feedbacks, their impact on surface temperature, glacier retreat, sea level rise, soil fertility, precipitation patterns, etc. are directly related to the number and quality of measurements (both spatially and temporally) that are input into these models. It is the combination of observation and modelling that holds the promise to improve monitoring and forecasts, but also further checks on the quality of observations (Chahine et al., 2006; Hertel et al., 2007a).

\subsection{Correction of bias due to spatial or temporal gaps in sampling}

Aerosol optical depth (AOD) has recently become a prominent target for assimilation work, both as a component needed to calculate aerosol radiative forcing and as a parameter linked through transfer functions to surface concentrations of particulate matter. Satellite measurements of AOD miss two-thirds of the Earth because of cloud cover and they have difficulties in correcting for land albedo. Sun photometers are rare in oceanic regions. As an example, Aeronet data from the year 2000 show a mean global AOD of 0.178 , the MODIS collection 5 derived AOD at these stations is slightly higher (0.199), but the global all-area average MODIS AOD is only 0.147 (for 2001) due to inclusion of clean oceanic regions. A corresponding median model constructed from the AeroCom models (Kinne et al., 2006; Textor et al., 2006) shows slightly lower AOD (0.164) at Aeronet stations and a global mean value of 0.111, all in all a considerable bias. Assimilation of satellite data reduced the error of modelled daily mean AOD against sun photometer data down to $0.03 \pm 0.06$ in AOD for the INDOEX campaign (Collins et al., 2001; Rasch et al., 2001). Assimilated AOD were better correlated with Aeronet measurements than either MODIS retrievals or GOCART simulations alone (Yu et al., 2003). Generoso et al. (2007) showed that a forward model missed boreal fire aerosols, while POLDER data-assimilated AOD fields 
had corrected this bias and showed even more realistic aerosol fields in polar areas where satellite data are sparse. The EU project GEMS implemented a 4D-VAR assimilation for reactive gases, aerosol and air quality (Hollingsworth et al., 2008); for aerosol, trial assimilation based on instantaneous MODIS retrieved AOD resulted in notable improvements (Benedetti et al., 2009). The detection and compensation of biases in the input data is, however, critical for improving assimilation methods. For example, Matsui et al. (2004) showed that the bias between MODIS retrieved and sun photometer AOD over North America reappeared in the MODIS-assimilated AOD product.

\subsection{Flux retrieval}

Inversion techniques are used to retrieve emission fluxes (or any other flux such as deposition, vertical exchange or chemical formation and losses) of reactive gases and aerosols (e.g. Konovalov et al., 2008). Also, retrieving emissions in an inverse transport simulation correctly pointed to those areas where aerosols are predominantly emitted (Dubovik et al., 2008). The application of such techniques generally requires large computer resources because huge matrices need to be inverted. Adjoint models may relieve this constraint somewhat, but require considerable recoding efforts (Elbern et al., 2007). A specific problem arises from the fact that several other processes, often equally uncertain, relate the observed bulk quantity - e.g. a concentration or optical depth - to the targeted flux. Currently, the principal limitation of any assimilation technique is the estimation of model errors variances/covariances (often called B-matrix) that determine, together with the estimate of observation error variances/covariances (R-matrix), the overall quality of the assimilation products and even the statistical robustness of the procedure (e.g. Kahnert, 2008).

\subsection{Proxy data usage to complete chemical characterization}

The variance in proxy or bulk data also contains information on the underlying chemical composition. The relation between different chemical components in the atmosphere is at times sufficiently well described through state-of-the-art chemical models. For instance, the assimilation of stratospheric compounds $\left(\mathrm{CH}_{4}, \mathrm{O}_{3}, \mathrm{HCl}\right.$ and $\left.\mathrm{H}_{2} \mathrm{O}\right)$ as observed by the HALOE satellite instrument was used to constrain other components of the stratospheric chemical composition (Lahoz et al., 2007). The resulting $\mathrm{NO}_{2}$ column values can be compared to ground-based DOAS measurements and show the potential of this method for improving the description of the overall chemical composition. The challenge for the design of an observing system is then to identify those chemical compounds that are easy to measure and contain sufficient information on other substances that are less attainable through direct measurement, like for example, $\mathrm{OH}$.

\section{Strategies for future atmospheric observation}

Atmospheric observation strategies need to recognize the many orders of magnitude difference in life-times of different chemical species in the atmosphere (Seinfeld and Pandis, 1998) that determine the temporal and spatial coverage required. Campaign-type observations generally target the microphysical understanding of complex systems, while the validation of models and trend assessment requires long-term observations that span several temporal scales. Both may require the vertical distribution of properties to be resolved. For example, an analysis of the spatial variability of ammonia/ammonium deposition concluded that a suitable observation strategy should consist of a network with a limited amount of supersites combined with a larger number of sites where low cost methods are applied, together with models for generalisation (Erisman et al., 2005). This study also pointed out the need for unified instrumentation. The following recommendations for observation networks emerged that are equally valid for deposition, aerosol and trace gas monitoring:

- The network has to provide (deposition) data for areas that are impacted (by deposition). This argues for observations close to the source as well as at remote receptors.

$\circ$ Measurements are needed at the most important and sensitive ecosystems.

○ A network must take into account the geographical distribution of ecosystem types. Both the pollution climate and average meteorological conditions should be different.

Here, we will consider the value of models for atmospheric observation strategies, as well as identifying a number of gaps in present observation networks. Following the identification of gaps, potential ways forward are highlighted.

\subsection{Lessons learnt from model-measurement comparisons}

The chain of processes linking emissions, atmospheric dispersion, chemical transformation and loss from the atmosphere of air pollutants is complex. Observations can typically address only a small portion of this chain, and models are thus an essential tool for our understanding of the atmosphere, and for generalising observations made at limited spatial and temporal scales. A comparison of model predictions against observed values is essential to verify our understanding of the behaviour and fate of atmospheric pollutants and for identifying weaknesses and gaps in both, models and measurements. The following examples are intended to illustrate the benefit of and the need for both model improvement and a more accurate observation of the natural atmosphere.

\subsubsection{Nitrogen partitioning}

Oxidised nitrogen exists in many forms in the atmosphere, including $\mathrm{NO}, \mathrm{NO}_{2}, \mathrm{HNO}_{3}, \mathrm{NO}_{3}, \mathrm{~N}_{2} \mathrm{O}_{5}$, PAN and both fine and coarse particulate nitrate. Long-term measurements are typically available for just $\mathrm{NO}_{2}$, or 'total' nitrate (gaseous $\mathrm{HNO}_{3}+\mathrm{NO}_{3}^{-}$), as measured by filter-packs, and wet-deposition components (Fagerli and Aas, 2008). At present, only two sites use denuders to allow for proper distinction of $\mathrm{HNO}_{3}$ from particulate nitrate in Europe. Although the recent deployments of AMS instruments bring a large amount of new data on fine nitrate levels, such instruments do not measure $\mathrm{HNO}_{3}$ and coarse nitrate, and so do not allow for a mass closure. Models suggest that gaseous $\mathrm{NO}_{3}$ and $\mathrm{N}_{2} \mathrm{O}_{5}$ may be important for tropospheric processing (Riemer et al., 2003), but measurements have been confined to a few campaigns over North America and the Atlantic (e.g. Brown et al., 2007; Sommariva et al., 2007, and references therein). These studies show much higher concentrations aloft than at the surface, highlighting the problem of surfacebased measurements and they demonstrate significant differences between model and observation. Thus, the importance of $\mathrm{NO}_{3}$ and $\mathrm{N}_{2} \mathrm{O}_{5}$ for VOC degradation, aerosol formation, and the nitrogen cycle in general is very uncertain, and the lack of observations of the speciation of oxidised nitrogen is a crucial limitation for understanding $\mathrm{NO}_{\mathrm{y}}$ chemistry and trends in Europe.

\subsubsection{Organic aerosol}

There are numerous reasons why it is difficult to model organic and secondary organic aerosol (OA and SOA), including our fundamental lack of understanding of the processes behind SOA formation (e.g. Donahue et al., 2005; Hallquist et al., 2009), and the range of possible precursor species or pathways. In addition, the emissions of 
precursor compounds are very poorly known. Isoprene and terpene emission estimates cover a range of factors of $2-3$, or even higher for specific regions and times (Guenther et al., 2006). Emissions of OA and EC especially from biomass burning are notoriously difficult to evaluate, not least because of the semi-volatile nature of most primary organic particulate matter (Robinson et al., 2007). Consequently, major discrepancies between model and observation are the rule rather than the exception. Chung and Seinfeld (2002) found major discrepancies of a factor of 10 or more at remote sites for OC. Volkamer et al. (2006) showed that models underpredicted SOA by factors of 3 (Mexico City) to 10-100 in more remote regions. The global model of Spracklen et al. (2008) underpredicted observed OA by a factor of 5-20 over oceanic regions. Simpson et al. (2007a) found good agreement between modelled and observed OA at Northern European sites, but poor agreement in southern Europe.

Clearly, emission studies performed under ambient conditions are urgently needed. Tracer compounds such as levoglucosan, potassium, acetonitrile, cellulose, sugars, or radiocarbon $\left({ }^{14} \mathrm{C}\right)$ may provide powerful diagnostics. For example, in the CARBOSOL project (Legrand and Puxbaum, 2007), Simpson et al. (2007a) used levoglucosan to show that much of the discrepancy found for modelled versus observed OA at two sites in southern Europe could be attributed to missing biomass burning sources. This study also showed that the model's SOA predictions were much lower than those derived from the source-attributed OA estimated by Gelencsér et al. (2007).

In addition, while carbon-14 analysis points to a large biogenic fraction of organic carbon and especially of its water-soluble fraction (Szidat et al., 2006; Weber et al., 2007), the latter appears to be highly correlated with anthropogenic tracers (Weber et al., 2007). Possibly, biogenic VOCs are indeed the major precursors for SOA, but (anthropogenic) oxidants are needed to transform these biogenic VOCs into SOA.

New, faster measurement techniques, and wider application of known techniques, are obviously required to disentangle the various components of ambient $\mathrm{OA}$, and to distinguish man-made from biogenic sources, and primary from secondary.

\subsubsection{Validation of emissions}

The emissions used in models are typically constructed by applying emission factors to activity statistics such as vehicle-km, or oil-and-coal usage. Such emission factors are often the result of close-to-source or laboratory measurements (e.g. dynamometer tests for vehicles), and the real-world emissions, especially for particulate emissions of OA may differ significantly (Robinson et al., 2007). Indeed, Robinson et al. (2007) suggested that rather than measuring particulate matter (PM) emissions only, the real need was for volatility-corrected VOC and PM emissions, properly validated for all regions of the globe.

For some compounds, notably $\mathrm{NO}_{\mathrm{x}}, \mathrm{CO}$ and isoprene, satellites have proved to be very useful for validation of emissions (Konovalov et al., 2008 and references therein). Konovalov et al. (2008) compared the trends in $\mathrm{NO}_{\mathrm{x}}$ emissions as reported to EMEP with the $\mathrm{NO}_{\mathrm{x}}$ trends derived from GOME and SCIAMACHY data and found good agreement for some countries such as France, Germany and Great Britain, but very large discrepancies for others. Although uncertainties remain in the interpretation of satellite data, this type of study has clear potential to verify reported emission data, and an extension of such studies to other pollutants would be very valuable.

\subsection{Gaps in a comprehensive observation system}

Despite substantial improvements in the last decade in the development of Earth observing tools, there is still a crucial need for both consolidation of the existing observation infrastructure and development of a more efficient observing system which would narrow major data and information gaps, and assist stake holders in planning new investments. There may be more than one approach to narrow the identified gaps, and the cost for a straightforward approach may be prohibitive or there may be technical limitations in the way of closing gaps. These (known) limitations in our ability to realize the desired system also tend to influence our definition of the desired system. In the case of atmospheric observations, there is some consensus that the desired observing system should provide well documented, global observations of the chemical composition and physical properties of the atmosphere from pole to pole with temporal, horizontal and vertical resolution sufficient to:

- satisfy and verify current legislation,

- validate and help to improve our understanding of atmospheric processes,

- understand the relationship between the atmosphere, the oceans and the land masses,

- permit accurate predictions of future atmospheric states by providing inputs to forecast models,

- support answering new questions that will only emerge in future.

Consequently, the key questions to address in terms of evaluating existing observation strategies are:

- Is there sufficient global coverage at adequate temporal and spatial resolution?

- Is the breadth of chemical and physical properties observed sufficient?

- Are the observations sufficiently well documented to be of long-term use?

Numerous papers discuss optimal network design strategies. Fuentes et al. (2007) gave a good overview and applied a Bayesian entropy approach for optimal network design for the North American ozone network. Janis et al. (2004) used Monte Carlo resampling techniques to derive optimal station densities for climate monitoring (temperature, precipitation) in the U.S. Sarigiannis and Saisana (2008) used inverse modelling and combined a series of priority pollutants with the objective of optimizing the cost-effectiveness of the observation network. Approaches such as this are also viable for other parameters.

In reality, observation networks are rarely designed from scratch. Rather, they develop with time. Atmospheric observations often start in a university setting for specific science questions and whenever an opportunity arises. There is a lack of long-term strategic planning that would likely lead to a more optimal, and perhaps more cost-effective, network design. In addition, optimal network design suffers from a certain lack of political 'forcing' and long-term commitment for monitoring activities because of the slow return-on-investment (e.g. compared with campaigns). Globally, the density of observations in any given country is typically related to the economic power of that country. On the one hand, countries are generally reluctant to invest in observations in other countries, even though a more homogeneous coverage would certainly improve the usefulness of all observations. On the other hand, there is an understandable reluctance to abandon existing long time series in favour of improving coverage in other areas.

Clearly, current gaps in the observing system for atmospheric composition changes are still numerous. In the following, we will review the major gaps identified for the in situ networks, but also for ground-based and space-based remote sensing platforms. Obviously, gaps do not only lie in the technical development but also in the data diffusion and assimilation. 


\subsubsection{Gaps resulting from a lack of network integration}

Progress in the understanding of atmospheric composition changes and its drivers is linked to the availability of advanced research infrastructures and networks. The previous chapters have shown that new methodologies for the investigation of atmospheric mechanisms, and the generation of original and high quality data for modelling purposes relied on the use of advanced facilities such as operational research aircraft, and/or monitoring stations. The coordinated efforts in the last decade allowing access to these research infrastructures, diffusion of information, and shared use of databases from a number of facilities have been extremely beneficial and have set the base for major achievements in atmospheric research over the last decades. In fact, the importance of data quality, quality assessment and quality control of observations has long been recognized among scientists, and many individual observation networks have been developed based on common data quality objectives and protocols (EANET, 2000; NDACC, 2008; WMO, 2007 and http://www.unece.org/env/ documents/2009/EB/ge1/ece.eb.air.ge.1.2009.10.e.pdf for EMEP). Thus, today reasonable harmonization exists within specific networks, but not necessarily between them (Hertel et al., 2007b; Klausen et al., 2003). The monitoring of aerosols in Europe, outside of urban areas, is an instructive example of the need for integration of networks. In 2006 there were more than 200 sites spread around Europe measuring various aerosol properties coordinated under different networks. A map showing these sites and their locations is displayed in Fig. 18.

These aerosol networks are partly a result of expansions of existing monitoring networks, and partly networks focusing solely on specific variables. Some of these networks originate from policy frameworks (EMEP) while others are based on other conventions (GAW), EU projects (EUSAAR, EARLINET) or other initiatives like AERONET. As a result, several international monitoring networks collect and apply remote sensing and in situ aerosol observations, in a relatively uncoordinated manner. It is striking that sites for ground-based in situ measurements rarely overlap with sites for ground-based remote sensing of the aerosol column. The same holds for the observation of AOD by sunphotometry and the aerosol vertical profile by lidar. Currently, the integration of data from both instruments is done more or less ad hoc. Clearly, there is a need for more stations to co-locate these techniques and use observations of one as a constraint in the interpretation of the other.

Concurrent and co-located measurements of key atmospheric species of interest to air quality and climate studies are extremely beneficial for assessing atmospheric composition, deposition to ecosystems, source apportionment and closure studies in relation to climate forcing. The concept has been promoted by EMEP and GAW in their recent monitoring strategies. It has been implemented as part of the PM supersite program in the USA that led to significant advancements in measurement methods, atmospheric characterization, and understanding of source-receptor relationships. Usually, atmospheric variability cannot be interpreted on the basis of observations of a single component. It is therefore of interest to all parties (scientific community and funding agencies) to promote establishment of shared stations belonging to two or more networks.

Ideally, such sites are located in such a way as to capture the range of environmental conditions in a given area. There is a need to

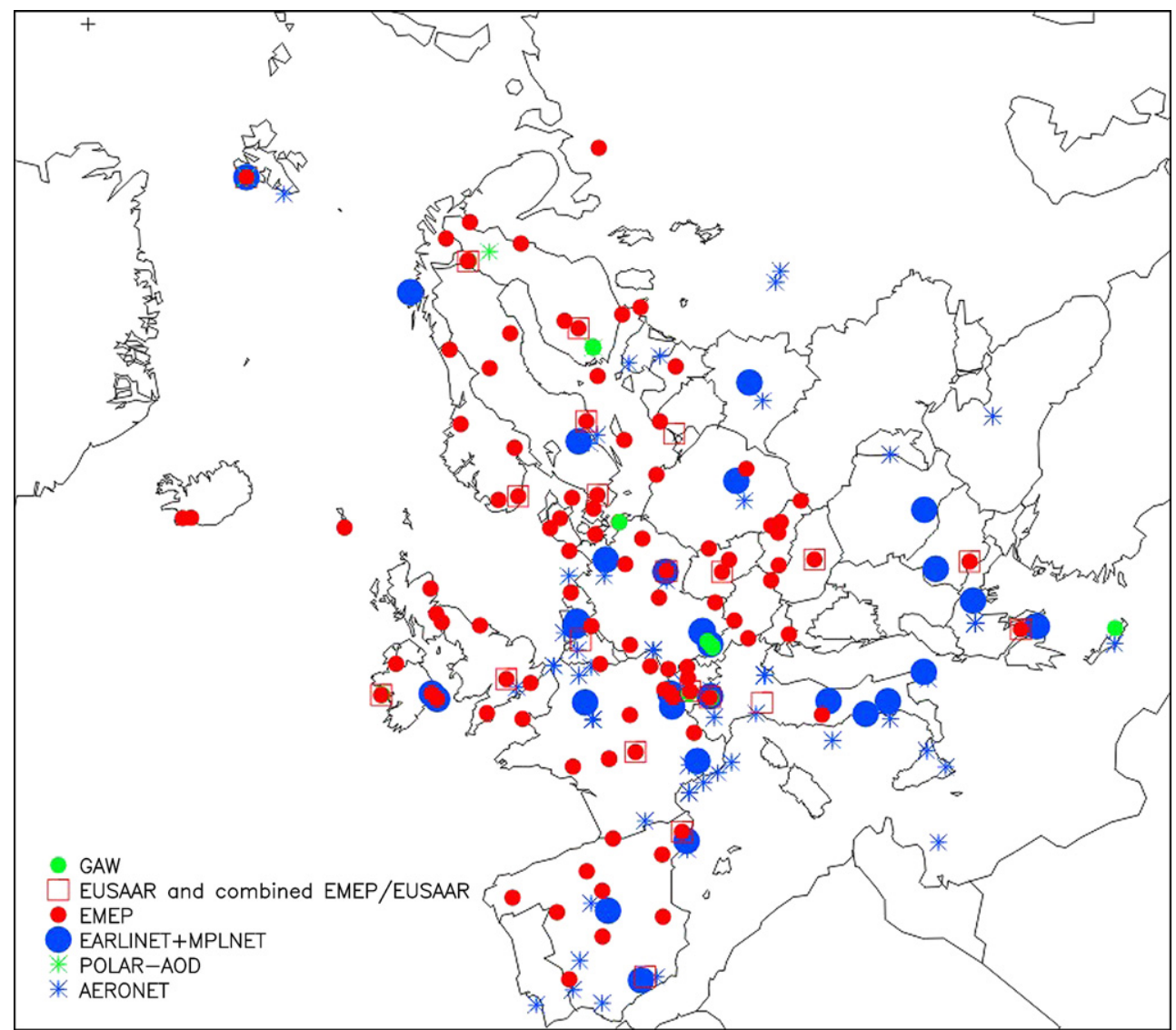

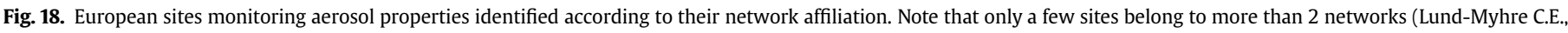
NILU, personal communication). 
carry out continuous and simultaneous ground-based observations of the atmospheric column using a suite of passive and active sensors adequately calibrated, including millimetre cloud radar, Raman lidar, infrared interferometers, sun photometers and sky imagers, and to couple this information to a more comprehensive characterization of in situ properties of the gaseous and particulate components of the atmosphere. Collocated lidar and sunphotometer instruments should be operated in a coordinated manner such that the night-time lidar observations of particle extinction and backscatter coefficients are still ongoing when the backscatter-ratio observations of the sunphotometer commence during sunrise; the same strategy, in reverse order, should be followed during sunset (Kolgotin and Müller, 2008).

Coupling mountain sites (for free tropospheric in situ sampling) to information on the atmospheric column cannot be implemented in all areas but has a strong potential to provide useful constraints on remote sensing instruments from the ground and consequently to contribute to validation of satellite products.

\subsubsection{Gaps resulting from uneven spatial coverage of observations}

Uneven global and regional coverage of atmospheric observations is due to geographical, economical and political/legislative reasons. In the past, the main focus of atmospheric observations has been on Europe and North America, and marine territories traditionally influenced by these. AOD sites of EARLINET, GAW, and AERONET are quite evenly distributed across the whole of Europe. In contrast, monitoring of gaseous and particulate species is fairly comprehensive across Northern and Central Europe, but gaps exist in Southern Europe as well as in the new EU member states in Southeast Europe. Concerning information on aerosol vertical profiles measured by lidar, the networks have gaps in Eastern Europe and Great Britain. While the main problem in Western and Northern Europe as well as North America thus appears to be one of network integration, in other parts of the world it is the very limited coverage of entire areas. Networks in continental Asia are improving but the area is still undersampled. Clear gaps remain in polar and equatorial regions, and generally in the southern hemisphere (Henne et al., 2008a,b; IGACO Theme Team, 2004; Nisbet, 2007). This is partly due to the predominance of oceanic surfaces but also due to the lack of monitoring stations on land and the restricted number of dedicated field campaigns in many parts of the Southern Hemisphere. A strong recommendation is to foster partnership between Europe and countries in the Southern part of Asia, America and Africa to ensure joint implementation of monitoring networks in these areas.

One of the drawbacks of ground-based networks is the limitation to land surfaces. Although data are generated at a few island sites, monitoring over oceans, which cover about $70 \%$ of the globe, is sparse. To improve this, the Maritime Aerosol Network (MAN), for example, was created to complement the AERONET sampling over land. MAN supplies column information on AOD, Ångstrom parameter and water vapour over data-sparse oceanic regions with hand-held sun-photometers. An important issue here is a strong link to other calibrated instruments.

Even spatial density of sampling sites is not the only relevant parameter to assess the adequacy of a network - rather, it is the representativeness of an observation for a given environment and for a given period that needs to be assessed. In the following, we will review the concept of representativeness and discuss ways to improve spatial coverage.

5.2.2.1. Assessment of the representativeness of in situ observations. In situ measurements characterize a particular volume of air at a particular point in time and space, hence - as a function of time - the particular location where the sampling takes place.
Consequently, the choice of location and whether and when it is adequate to answer particular questions is critical. The issue of representativeness of in situ observations is central in the identification of gaps in observation strategies.

Representativeness has been defined as follows: "the area in which the concentration does not differ from the concentration measured at the station by more than a specified amount can be called the area of representativeness of the station" (e.g. Henne et al., 2008a,b; Larssen et al., 1999). A slightly different approach is to define a point-to-area representativeness, where a point measurement $q$ is representative of the average $Q$ in a larger volume if the probability $\operatorname{Pr}$ of the squared difference between point and volume is smaller than a certain threshold $\delta$ for more than $90 \%$ of the time (Nappo et al., 1982).

$\operatorname{Pr}\left[(q-Q)^{2} \leq \delta^{2}\right] \geq 0.9$

Basically this repeats the statement of Larssen et al. in a more quantitative form. The maximum tolerable difference $\delta$ has to be assessed for every individual variable; it should not be smaller than the uncertainty of the measurement. In addition, the area (or volume) of interest will vary with application. For the inter-comparison of in situ (point data) and model simulations or remote sensing data (volume data) and for data assimilation purposes, the measurements need to be representative in the sense of the definition given above to successfully derive meaningful results. Typical spatial scales for representativeness based on subjective experience range from metres (for polluted traffic sites) to hundreds of kilometres for background remote sites. Properties with large spatial variability need spatially dense networks, and properties with large temporal variability need more frequent measurements. For instance, an atmospheric column property measured at a mountain-top is likely to underpredict the regional average, whereas a locally polluted site is likely to overestimate the regionally averaged column amount.

For the assessment of regional representativeness or biases (e.g. orography, pollution), regional averages derived from satellite observations at different spatial scales (e.g. 1, 3 or 10 deg lat/lon) can be related to those at a local scale (e.g. $10 \mathrm{~km}$ ), at times when data at the local scale are retrieved. As long as the ratio stays close to 1 , the local data are representative for the larger scale. The overall retrieval accuracy is less of an issue, since differences can be examined. For the aerosol optical depth (AOD) many satellite data sets with spatial pattern information are offered. The multi-annual (2000-2007) MISR data set seems particularly useful, because it offers global coverage (snow and ice covered areas excluded). For the regional assessment with satellite data both the average relative error $(E=\Sigma(\operatorname{abs}($ regional - local $) /$ local $) / \Sigma \#)$ and the average relative bias $(E=\Sigma$ ((regional - local)/local $) / \Sigma \#)$ are determined on a monthly and seasonal basis. Then, based on particular thresholds for the error and bias all sites are rated. For other properties, such as aerosol composition data a similar approach could be applied by looking at the consistency of how pre-defined aerosol models in satellite retrievals are chosen. Once scores for quality and representativeness are in place, (statistics of) similar data of different locations can be combined. In order to give modelling a hand, it seems desirable to place these data on a regular grid to simplify comparisons with model simulations. Examples of such $1 \times 1$ lat/lon gridded products for mid-visible AERONET properties for AOD and absorption AOD and Ångstrom parameter on a seasonal basis are given in Fig. 19.

5.2.2.2. Monitoring sites and field campaigns near emission sources. Apart from regionally representative observations, measurements close to sources, in particular the so-called mega cities, are needed both from a health perspective and in order to improve 

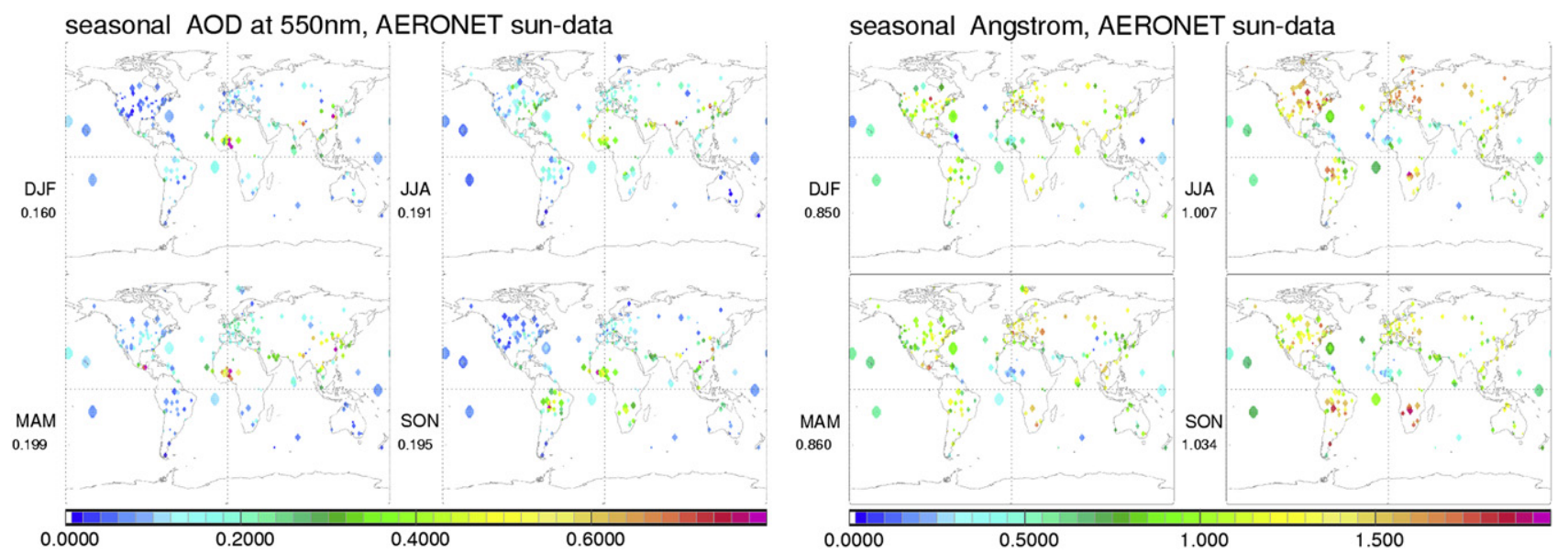

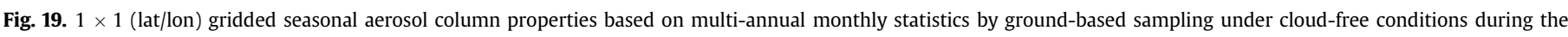

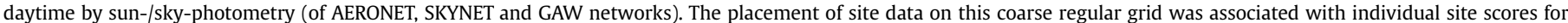

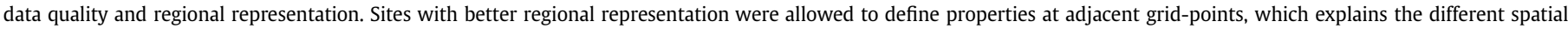
domains associated to individual sites. Plots are provided for mid-visible properties (550 nm) of AOD (left), and Ångström parameter (right)

emission estimates. There is a lack of atmospheric observations particularly in Eastern Europe, Russia and the Tropics (Nisbet, 2007). If such observations exist, they are not included and recognized by international monitoring programs and are often of unknown quality (e.g. the Russian filter instruments for ozone and AOD).

China and India, in particular, are emerging economies with hugely increasing emissions. Both countries as well as the whole of Africa are severely undersampled, and the most consistent information currently comes from satellite observations. Unfortunately, the Tropics in particular are characterized by very frequent cloud coverage. This makes satellite observations difficult and less reliable, and calls for enhanced efforts related to in situ measurements, both long-term and intensive campaigns. The Bodele Experiment (BodEx) (e.g. Giles, 2005; Todd et al., 2007) in the north of Chad in Africa showed the importance of such field missions close to the sources to avoid the impact of mixing of emissions with pollution in the boundary layer downwind.

Concerning mineral dust in Africa, this means investigating more eastern areas of the Sahara because the chemical composition of mineral dust changes from the northeast corner to the southwest. The eastern Sahara is also important because dust is frequently transported from that region to Southeast Europe. Mineral dust transported from the Arabian Peninsula across, e.g. the northern Indian Ocean could be the focus of future activities, as well as desert areas in central Asia such as Gobi and Taklamakan. Most present data are from observations downwind of the sources where mixing with anthropogenic pollution has already occurred. The Asian Dust Lidar Network (ADnet) (Sugimoto et al., 2005) provides geometrical and optical parameters of Asian dust plumes, but the data products are still rather limited. In fact more data on dust from various areas of China upwind of the strongly populated coastal areas are needed.

Next to mineral dust, forest fires and the burning of grassland is an important large-scale natural source of aerosol particles and gases. Most of our data on forest-fire smoke in boreal areas come from long-range transported smoke plumes, observed thousands of kilometres downwind of the fire sources. EARLINET data (Mattis et al., 2008) have demonstrated the need for going much closer to the source regions in the framework of dedicated field missions. Forest-areas in Siberia are a blind spot that the lidar network CisLiNEt (Chaikovsky et al., 2008) does not cover adequately due to the sparse number of lidars in the network and the forested area in Siberia and Western Russia. Satellite data lidar observations in southeast Europe have also indicated that the burning of agricultural waste must generate enormous amounts of aerosol particles and gases but emission rates and composition, in particular for the aerosol fraction, are poorly known.

5.2.2.3. Measurements in the free troposphere (mountain sites, aircraft). Many atmospheric variables have strong vertical profiles as a result of dynamic or chemical processes and a strong vertical distribution of sources and sinks (e.g. most sources are at ground level). The transformation of column density into in situ concentrations requires information on the vertical structure of the atmosphere. Aircraft and balloon observations present an opportunity for characterizing vertical profiles during ascent and descent, but they usually provide poor temporal coverage, insufficient for long-term trend analysis (Thompson et al., 2004).

Data from fixed platforms at various elevations have been combined successfully to reconstruct vertical profiles (Schaub et al., 2006). Fixed platforms at higher elevations are, however, often influenced by the diurnal or seasonal variation of the planetary boundary layer (PBL) height, an important parameter that characterizes the vertical structure of the atmosphere. Injection of PBL air into the free troposphere by thermal convection (mountain venting) has been documented to be a highly important mechanism of vertical exchange for alpine sites (Henne et al., 2005). Hence, approaches to identify free tropospheric air include concurrent measurements of species with well defined vertical profiles $\left(\mathrm{H}_{2} \mathrm{O}\right.$, $\mathrm{O}_{3}, \mathrm{NO}_{\mathrm{x}}$, etc) and diurnal variations, as well as the use of models. Because of its importance, cheap methods for determining the variations in PBL extent are still needed.

Observations of the free troposphere are needed to understand long-range transport, climate change, trends in the oxidizing capacity of the atmosphere, and as a 'baseline' for the characterization of atmospheric chemistry and physics (IGACO Theme Team, 2004; WMO, 2007). GCM-CTMs have advanced to the point where they are able to predict 'background' concentrations adequately, but miss events. These are relevant for air quality. There are large gaps in our understanding of the role that hemispheric transport plays in regional air quality (http://www. htap.org). Continuous, long-term measurements of the free troposphere - spatially more representative than observations from within the boundary layer - are also needed for satellite validation. Key compounds include PAN, NMHCs, OVOCs, BC and various other aerosol variables. 
Intensive aircraft missions provide important free tropospheric data and can help in the validation of satellites, but they are not useful to identify long-term trends. Routine aircraft missions such as CARIBIC (Brenninkmeijer et al., 2007) and MOZAIC provide extremely valuable data sets of a number of key species with sufficient measurement frequency that will be more useful to this end. Even though the certification of measurement equipment aboard commercial airliners is a huge burden, limiting the ability to adapt to emerging observation techniques, both projects continue for now as part of the preparation phase of a proposed new research infrastructure IAGOS-ERI (Volz-Thomas and the IAGOS Team, 2007, http://www.iagos.org, http://cordis.europa.eu/esfri/). Through IAGOS-ERI, observation capabilities are extended to include cloud particles, aerosols, nitrogen oxides $\left(\mathrm{NO}_{\mathrm{x}}\right)$, and greenhouse gases $\left(\mathrm{CO}_{2}\right.$ and $\left.\mathrm{CH}_{4}\right)$ with a compact package of airborne systems. IAGOS also explores new technologies like cavity ring down spectroscopy to build a future generation of a very small instrument package $(<20 \mathrm{~kg})$ measuring several species. With improved transmission, availability and usability of data, routine aircraft observations will be fully integrated in the GMES Atmospheric Service. IAGOS promises to narrow the gaps in free tropospheric observations of key species, however the size of the fleet will largely depend on the availability of long-term funding.

\subsubsection{Gaps in in situ observations}

In situ observations are still the back-bone of long-term atmospheric monitoring. Specific challenges exist for an observing system that aims to characterize atmospheric states with regional resolution and global coverage, and to support predictions of future changes:

- The long-term stability of measurements is especially critical for short-lived species such as aerosols and certain reactive gases, because changes in anthropogenic emissions will have a significant effect on regional climate evolution. To detect such trends requires high precision and accuracy.

- Historical measurements are crucial for attribution studies. However, their quality is often poorly documented and their interpretation therefore difficult. For example, higher detection limits in early records may introduce a positive bias in reported concentrations.

- Records of at least 10 years are needed to relate atmospheric forcing components to observed changes in climate. This requires a long-term measuring capacity.

- Long-term intercomparison of instruments across continents and countries and traceability to a common standard are needed since climate effects are generally global and require a global description of atmospheric composition.

- Atmospheric measurements require very specific expertise and data processing. The multitude of methods and operators has prevented the appearance of simple databases, and data quality, storage and availability need to be improved.

- Wet removal is dominant for a large part of the aerosol particle spectrum but also of key reactive gases (see Fowler et al., in this issue), in particular the anthropogenic fraction. Wet removal has not been the focus of recent intensive studies and would deserve to be revitalized.

With these considerations in mind, we can identify major gaps in current observing capacity for in situ measurements of gases and aerosol species.

5.2.3.1. Gaps in in situ gas measurements. Table 4 lists the measurements recommended by GAW and EMEP and identifies the key properties and chemical variables governing atmospheric
Table 4

List of gas measurements recommended by GAW and EMEP (WMO, 2007). The detailed list of VOCs recommended can be found in WMO (2007).

\begin{tabular}{|c|c|c|c|c|}
\hline \multirow[t]{2}{*}{ Property or chemical variable } & \multicolumn{4}{|l|}{ Context } \\
\hline & $\begin{array}{l}\text { Air } \\
\text { quality }\end{array}$ & $\begin{array}{l}\text { Oxidation } \\
\text { efficiency }\end{array}$ & Climate & $\begin{array}{l}\text { Strat. ozone } \\
\text { depletion }\end{array}$ \\
\hline $\mathrm{O}_{3}$ (ozone) & $\overline{\mathbf{a}}$ & $\mathbf{\square}$ & $\mathbf{\square}$ & $\mathbf{\square}$ \\
\hline CO (carbon monoxide) & $\mathbf{\square}$ & $\mathbf{\square}$ & & \\
\hline$j\left(\mathrm{NO}_{2}\right)$ & $\mathbf{a}$ & $\mathbf{\square}$ & & \\
\hline$j\left(\mathrm{O}^{1} \mathrm{D}\right)$ & & $\mathbf{\square}$ & & \\
\hline $\mathrm{H}_{2} \mathrm{O}$ (water vapour) & $\mathbf{\square}$ & $\mathbf{\square}$ & $\mathbf{\square}$ & $\mathbf{\square}$ \\
\hline $\mathrm{HCHO}$ (formaldehyde) & $\mathbf{\square}$ & $\mathbf{\square}$ & & \\
\hline VOCs (volatile organic compounds) & $\mathbf{\square}$ & $\mathbf{\square}$ & & \\
\hline Active nitrogen: $\mathrm{NO}_{\mathrm{x}}=\mathrm{NO}+\mathrm{NO}_{2}$ & $\mathbf{\square}$ & $\mathbf{\square}$ & & $\mathbf{\square}$ \\
\hline Reservoir species: $\mathrm{HNO}_{3}$ & $\bar{\square}$ & $\overline{\mathbf{n}}$ & v & $\overline{\mathbf{a}}$ \\
\hline $\mathrm{N}_{2} \mathrm{O}$ & & & $\mathbf{\square}$ & \\
\hline $\mathrm{SO}_{2}$ & $\mathbf{\square}$ & & $\mathbf{\square}$ & \\
\hline Active halogens: $\mathrm{BrO}, \mathrm{ClO}, \mathrm{OClO}$ & & & & $\mathbf{\square}$ \\
\hline Reservoir species: $\mathrm{HCl}, \mathrm{ClONO}_{2}$ & & & & 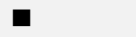 \\
\hline $\begin{array}{l}\text { Sources: } \mathrm{CH}_{3} \mathrm{Br}, \mathrm{CFC}-12 \text {, } \\
\text { HCFC-22, halons }\end{array}$ & & & $\mathbf{\square}$ & $\mathbf{\square}$ \\
\hline $\mathrm{CO}_{2}$ & & & & \\
\hline $\mathrm{CH}_{4}$ & & $\mathbf{\square}$ & $\overline{\mathbf{a}}$ & $\mathbf{\square}$ \\
\hline
\end{tabular}

composition. Comprehensive monitoring of these by in situ and remote-sensing techniques is deemed crucial for our understanding of atmospheric processes and trends.

Rapid increases in industrial pollutants over Asia (Richter et al., 2005), and trends in background surface $\mathrm{O}_{3}$ e.g. in the Eastern Pacific (Jaffe et al., 2003; Parrish et al., 2004) and in Switzerland (Brönnimam et al., 2002; Ordoñez et al., 2005) have been observed. Ozonesonde data (e.g. from Boulder, Colorado, U.S.A.) do not exhibit these long-term changes (Oltmans et al., 2006), however, it is recognized that the limitation to weekly observations reduces the ability to detect trends, e.g. (Jaffe and Ray, 2007; Weatherhead et al., 1998). It is still unclear if these trends are linked to changes in Asia, climate, or regional photochemistry.

For long-lived species (e.g. $\mathrm{CH}_{4}, \mathrm{CO}_{2}$ ), the advent of recent satellite missions (in particular IASI and GOSAT) may satisfy the need for global coverage for the next decade. However, a full understanding of the global carbon cycle requires continuous observations to characterize the diurnal variation of sources and sinks. The upcoming ICOS project (http://icos-infrastructure.ipsl. jussieu.fr/) will provide significant harmonization of efforts.

Concerning reactive gases, the current suite of satellites includes sensors for the observation of a large number of relevant oxygen, nitrogen, sulphur, hydrogen, carbon and halogen species (http:// accsatellites.aeronomie.be/Missions.html). For some of these species, the diurnal variation is so substantial (e.g. because it is due to photochemical production and destruction) that a process-level understanding of the physicochemical behaviour requires continuous observations with at least hourly resolution. At present, this is only provided by ground-based instrumentation and the density of the networks varies a great deal geographically as well as by parameter. Satellites can be of value in tracking long-term changes (Brunner et al., 2006; Fioletov et al., 2002; Konovalov et al., 2008), but there is an inherent uncertainty related to sensor drift and the limited possibilities for regular calibration that is very difficult to overcome.

At present, trends in oxidants are difficult to evaluate, the effects on health and ecosystems are very large, and it is striking to see that very few countries operate monitoring systems enabling an evaluation of oxidant precursors $\left(\mathrm{NO}_{\mathrm{y}}\right.$-species and VOCs). Such measurements are required according to the EMEP and GAW monitoring strategies. The role of VOCs in secondary organic aerosol formation is a key one. To be most effective 
oxidant precursor measurements should, thus, be co-located at the EMEP-GAW joint supersites.

5.2.3.2. Gaps in in situ aerosol measurements. Aerosols are involved in many key processes governing, e.g. the atmospheric radiation budget, water cycle, and chemistry, which in turn affect human health, traffic systems, and development of ecosystems, but for lack of observational capabilities, the present understanding is insufficient to properly assess their role.

A list of comprehensive aerosol measurements with a subset of core variables (printed in bold) has been recommended by the GAW Scientific Advisory Group on Aerosols for long-term measurements in the global networks (see Table 5). GAW recommendations are similar to those provided by EMEP and emphasize the links between remote sensing and in situ data measurements.

The most obvious gaps in present networks are the CCN measurements, with only about 3 stations with a long-term commitment in the GAW network. It is hoped that the situation can be improved with the recent availability of a commercial $\mathrm{CCN}$ counter (Droplet Measurement Technology). A second major gap is related to measurements of $\mathrm{OC}$ and EC (organic and elemental carbon, two major chemical components), and the light absorption coefficient. Chemical discrimination between OC and EC presently yields highly different results depending on the method chosen. The current methods for determination of the light absorption coefficient depend on the deposition of the aerosol on filters and suffer from artefacts. Research is going on to provide appropriate correction algorithms.

Many aerosol properties depend on relative humidity. Hygroscopicity tandem differential mobility analyzers (HTDMAs), which measure the increase of the particle diameter as a function of relative humidity were developed more than 20 years ago, however, inter-comparisons of different instruments have only been performed recently (Duplissy et al., in press). The aerosol scattering coefficient varies even more strongly with relative humidity than the diameter, and adequate instrumentation has not been available in Europe until recently (Haywood et al., 2008).

While the long-term measurements mainly aim at providing a climatology and detecting trends, short-term campaigns are needed to characterize the underlying processes, in particular the identity, concentration, formation pathways and possible roles in affecting surface tension, optical properties, reactivity and $\mathrm{CCN}$ activity of SOA. Recent field and chamber studies suggest that complex organic matter contains organosulphates, amines, organic nitrates and other species that have not yet been well studied.

\section{Table 5}

List of comprehensive aerosol measurements with a subset of core variables (printed in bold) that are recommended by the GAW Scientific Advisory Group on Aerosols for long-term measurements in the global network (WMO, 2007).

\footnotetext{
WMO/GAW continuous measurement

Multi-wavelength optical depth

Mass in two size fractions

Major chemical components in two size fractions

Light absorption coefficient

Light scattering coefficient at various wavelengths

Hemispheric backscattering coefficient at various wavelengths

Aerosol number concentration

Cloud condensation nuclei at $0.5 \%$ supersaturation
}

\section{Intermittent measurement}

Aerosol size distribution

Detailed size fractionated chemical composition

Dependence on relative humidity

CCN spectra (various supersaturations)

Vertical distribution of aerosol properties
Analytical methods to extract, identify and quantify these compounds need to be improved. Quantitative field measurements at fairly low detection limits and characterization of these compound classes as well as of HULIS, WSOM, WISOM, nanoparticles, new tracers, functional groups etc. are needed. Techniques for high time resolution of more components, e.g. tracers of functional groups, and mobile platforms are needed to study aging processes and validate Eulerian type models.

Nucleation of new particles is recognized as a widespread phenomenon but the identity of the main nucleating agents and exact mechanisms of initiation and growth are not well known. The increased use of nano-materials also calls for improvements in this area. The minute mass of the nucleation mode particles presents a formidable challenge to observation and new instrumentation is urgently required. The missing parameters need to be studied in several different environments (urban, rural, remote locations) in order to adequately include nucleation in models.

As mentioned previously, little development in sampling and analysis of precipitation has been made over the past decades, despite the continuing need to quantify wet deposition to ecosystems. The best available technique for sampling is still to use 'wet-only' collectors that provide daily (or shorter) time resolution and maintain samples refrigerated to minimize chemical and biological degradation of the collected sample. However, this type of sampler is necessarily bulky and prone to under-sampling small wind-blown droplets. Therefore, networks of precipitation samplers more usually use bulk samplers, although these suffer from dry deposition of material onto the collector surfaces between precipitation events, and in general do not prevent degradation of the samples with time.

\subsubsection{Gaps in remote sensing from the ground}

5.2.4.1. Aerosol lidars. Lidar remote sensing is the most promising tool to address an observational gap regarding the vertical profile of aerosol optical and microphysical properties, and significant progress has been made. However, many parts of the world are undersampled; lidar technology needs to be improved and different observation techniques need to be better integrated. Intensive field campaigns in unexplored or not well covered regions closer to aerosol sources are of highest priority.

5.2.4.1.1. Measurements in the ultraviolet and infrared regions. To better resolve particle size distributions and to reduce the uncertainties of the retrieved microphysical parameters, present-day multi-wavelength Raman lidars need to expand into the ultraviolet and infrared range of the electromagnetic spectrum. Mie-scattering theory states that measurements at wavelengths on the order of the size of the investigated particles are needed to obtain size resolved information of the scattering particles. Size-resolved observations are presently limited to approximately 100-150 nm particle diameter at the lower end of the particle size spectrum, and recent tests suggest that the resolution of the size distribution for Aitken mode particles can be improved by extending observations to wavelengths as low as $266 \mathrm{~nm}$.

Results on particle size distributions for large particles mainly rely on measurement of sun radiation in the forward scattering regime (Dubovik and King, 2000). AERONET results are inverted to particle size distributions up to $30 \mu \mathrm{m}$ particle diameter (Dubovik et al., 2002). Lidar retrievals are presently restricted to particle effective radii less than $2 \mu \mathrm{m}$ due to the maximum available measurement wavelength of $1064 \mathrm{~nm}$ (e.g. Veselovskii et al., 2005) limiting the particle microphysical characterization to the finemode fraction (e.g. Müller et al., 2004; Böckmann et al., 2005; Veselovskii et al., 2002, 2004). Measurements at greater wavelengths may be possible, but the need to separate molecular 
scattering from the particle scattering is problematic in height ranges where molecular scattering is dominant. In addition, determination of the particle backscatter coefficient requires knowledge of the particle extinction-to-backscatter ratio, which varies significantly at wavelengths of 355 and $532 \mathrm{~nm}$ (Müller et al., 2007a,b). Extremely weak signals from Raman scattering on molecules need to be detected and call for instrumentation with increased sensitivity. Novel detectors for near-infrared wavelengths have recently become affordable and the concept appears attractive enough to be tackled in the coming years.

Significant deficiencies still exist in our ability to characterize the vertical profile of the liquid phase, to distinguish ice crystal habits, and to understand aerosol-cloud interactions. An increase in aerosol tends to decrease cloud drop size and increase cloud reflectance, but many questions remain concerning the importance and the controlling parameters of this process (Feingold et al., 2006). Multi-wavelength and multi-angle Raman lidar systems in combination with passive instruments hold a potential to address the modelling of the physical processes occurring at the base of clouds involving aerosols, and to retrieve information about condensed mass and hydrometeors size distribution for both the liquid and the ice phase.

5.2.4.1.2. New data products. The future generation of aerosol lidars will be able to provide new data products in addition to backscatter and extinction coefficients. In particular, the particle phase function can be obtained from so-called bi-static lidar systems. Although not a new data product in a strict sense, we also have to consider particle depolarization measurements at several wavelengths. At present, lidar systems in general only employ one measurement wavelength to infer the so-called linear particle depolarization ratio. Observations as part of the Saharan Mineral Dust Experiment (SAMUM) (Freudenthaler et al., 2009) suggest that an improved characterization of non-spherical mineral dust particles is possible, if we use at least two measurement wavelengths. Work in that regard has just begun, and it is not yet clear how many measurement wavelengths are needed, and in which range of the electromagnetic spectrum they should be situated. Finally, the detection of Raman scattering from aerosol particles could allow the detection of specific types of particles on the basis of their chemical composition. In this concept it is assumed that the wavelength of the Raman scattered signal is characteristic for different components. The first such measurements were made for Asian mineral dust (Tatarov and Sugimoto, 2005; Tatarov et al., 2006). However, the Raman scattered signals are extremely low, and it remains to be seen if this measurement technique works for particle types other than mineral dust.

5.2.4.1.3. Lidar measurement under daylight conditions. The majority of information on aerosol optical and microphysical particle parameters currently comes from observations at night time. Detailed studies on the diurnal variation of aerosol properties, the effects of mixing of air in the planetary boundary layer during sunrise and sunset, and comparisons with passive aerosol sensors onboard (sun-synchronous) satellites such as NASA's A-Train mission are presently rather limited. For satellite validation, AERONET sunphotometers are currently indispensable, and the daylight limitation severely constrains the value of aerosol Raman lidar measurements in this context.

5.2.4.2. Gaps in remote sensing of gases from the ground. Development of remote sensing capabilities for aerosol species should be complemented by similar developments for gaseous species. Progress is being made to optimise the current FTIR and MAX-DOAS networks for observations of tropospheric composition. Efforts are also ongoing to make the networks more consistent internally, e.g. by designing common retrieval strategies. Both networks should evolve in the next decade both in term of spatial representativeness and technological developments.

5.2.4.2.1. FTIR observation network. Present FTIR networks are working on the improvement of retrievals in the upper troposphere and lower stratosphere (UT/LS), on the detection of greenhouse gases, on the precision and the automation of the measurements, the standardization of the retrieval techniques, and the capabilities of observing under cloudy conditions.

Better constraints on the water vapour in the UT/LS range are important because of its impact on the radiative budget and to answer questions about the transport pathways of water vapour from the troposphere into the stratosphere. FTIR spectrometry is capable of retrieving vertical profile information up to the upper troposphere (Schneider et al., 2006a; Schneider and Hase, 2009), but it is a challenging, ongoing task. Ground-based solar absorption FTIR spectrometry can provide the total column of ozone with a precision that equals that of Brewer instruments ( $\sim 1$ DU) (Schneider et al., 2008a,b; Schneider and Hase, 2008). However, this requires careful control of the experiment (e.g. a high precision of the solar tracker) and the simultaneous retrieval of the temperature profile in the atmosphere, which is not straightforward. There is a need to implement these advanced techniques at more sites.

Progress is needed towards automation of the measurements because manual observation and retrieval is too costly, often not standardized, and too slow. In the meantime, several sites have implemented remote control or automatic operation of the observations (e.g. Neefs et al., 2007; Hannigan et al., in press), which has increased the number and sustainability of observations and enables contributions to the GMES Atmosphere Service.

The near-infrared spectral region offers advantages for trace gas measurements from both a spectroscopic and instrumental point ofview (e.g. Washenfelder, 2006). Recent work has shown that dry-air column-average $\mathrm{CO}_{2}$ and $\mathrm{CH}_{4}$ volume mixing ratios can be retrieved with high precision (Dufour et al., 2004; Yang et al., 2002; Warneke et al., 2005). The $\mathrm{CO}_{2}$ column abundance can be retrieved with a precision of $\sim 0.1 \%$ under clear-sky conditions (Washenfelder, 2006), and the tropospheric column-average $\mathrm{CH}_{4}$ volume mixing ratio with a precision of $\sim 0.5 \%$ (Dils et al., 2006). Column measurements are insensitive to vertical mixing and diurnal fluctuations in the boundary layer and models predict that a few column measurements at carefully selected sites could constrain the global carbon budget. Consequently, the objective of the Total Carbon Column Observing Network (TCCON), currently consisting of about 10 operational sites (http://www.tccon.caltech. $\mathrm{edu} /$ ) is to contribute to the evaluation of the global carbon budget and to support satellite experiments like SCIAMACHY and GOSAT that use the same absorption bands in the near-infrared.

Finally, the extent to which FTIR measurements can be extended beyond clear-sky conditions is being investigated. Slight perturbations of the sky during the recording of the interferogramme can be corrected based on a recording of the solar light intensity (Washenfelder, 2006). Efforts are ongoing to verify the applicability of this method in order to enable observations under more variable sky conditions.

5.2.4.2.2. MAX-DOAS observation network. The MAX-DOAS technique for passive trace gas remote sensing in the UV-visible region requires an optimal compromise between the number of angular directions sequentially observed during one scan and the time resolution of the measurements. A more systematic and rigorous assessment of the information content of the technique and the error budget are needed. These should involve appropriate correlative in situ, ground-based and airborne observations, the latter being needed to assess the profiling capabilities of the MAX-DOAS technique. Retrieval algorithms for trace gases and aerosols should 
be standardized between networks to facilitate use of common methods and tools and to improve comparability. The combination of MAX-DOAS measurements of the scattered sky light with direct-sun measurements is expected to improve the accuracy of total column measurements and therefore to provide better constraints on the vertical profile. More advanced radiative transfer models need to be developed for a better exploitation of additional parameters such as the Ring effect and the degree of polarization of the sky radiances that are (potentially) accessible to MAX-DOAS observations. Such measurements are expected to improve, in particular, the determination of aerosol content and properties (Wagner et al., 2004).

Some obvious gaps in spatial coverage of trace gas measurements could be alleviated by MAX-DOAS observations. For example, more measurements of $\mathrm{HCHO}$ and glyoxal are needed in regions with anthropogenic, biogenic and pyrogenic emissions. A larger number of MAX-DOAS systems should be deployed to improve the sampling of gradients in $\mathrm{NO}_{\mathrm{x}}$ emissions.

Strategies are needed for the deployment and better exploitation of MAX-DOAS instruments for the validation of satellite instruments like OMI, SCIAMACHY, GOME-2, etc. One of the main challenges in this regard is the measurement of spatial gradients, which are very often seen differently from space and from the ground (e.g. Leigh et al., 2007; Brinksma et al., 2008). The deployment of travelling instruments or small-scale networks may offer solutions and the potential of the technique to resolve not only vertical but also horizontal gradients in the measured trace gas concentration should be explored. For this, specific radiative transport models and adequate inversion techniques dealing with 3D effects will be needed.

\subsubsection{Gaps in the observations of tropospheric composition from space}

Section 3.6 above reported the list of species available from current UV-vis, NIR and TIR instrument observations. We are at the early stage of measuring some other reactive species, such as hydrocarbons, that are present in low concentrations and hence difficult to detect by satellite. Some of them are aerosol precursors and could be important for better understanding the processes behind SOA formation in different regions/conditions. Important molecules for which improvement in instrumentation is necessary to provide global distributions and knowledge of the photochemistry cycles include $\mathrm{NO}_{\mathrm{x}}$ reservoirs (e.g. PAN, ...), hydrocarbons such as isoprene and its oxidation products.

Spatial and temporal coverage are determined by the orbits from which the instruments are operating and also by their scanning mode. Satellites in polar Low-Earth Orbits (LEO, about $800 \mathrm{~km}$ altitude) provide global coverage within a day at best, with a maximum of two overpasses per day at a given geolocation for infrared sounders, which measure both during day and night, and one to two measurement per day for UV sounders. LEO satellites on drifting orbits or a constellation of LEO polar orbiting satellites are two means of improving the revisit time on the Earth. Geostationary orbits (GEO, about $36000 \mathrm{~km}$ altitude) would provide much higher temporal sampling but would also restrict the observations to only a part of the Earth. For meteorological applications, the combination of LEO and GEO instruments is nowadays considered to be the most appropriate solution to achieve a complete picture of the state of the atmosphere (temperature, wind, humidity...). The same is certainly true for observations of tropospheric chemistry which aim, amongst other, at tracking fast chemical processes. Therefore, several projects are currently being discussed to provide such synoptic measurements also for trace species.

Spatial resolution is determined by both instrumental parameters and the orbit. Current LEO instruments provide footprints of down to about $15 \mathrm{~km}^{2} \times 15 \mathrm{~km}^{2}$ at nadir for trace species. Further reductions in pixel size are limited by the signal to noise achievable from the number of photons observed, and are already close to what can be obtained by instruments given the limitations in size and orbit. Spatial resolution can be further improved by increasing integration time, which is possible from GEO albeit at a loss of spatial coverage. Aerosol observations do not need high spectral resolution and therefore can use data from hyperspectral imagers already offering much higher spatial resolution today.

The vertical information content in the troposphere is very limited in passive nadir UV/vis measurements in general. Measurements in the thermal infrared can be inverted to provide coarse vertical profiles, the quality of which depends strongly on the spectral resolution of the measurements. A vertical resolution of typically $6 \mathrm{~km}$ is achievable with current TIR space sounders for $\mathrm{O}_{3}$ and $\mathrm{CO}$, which could be improved to 4-5 km with more advanced TIR instruments. By combining measurements in different wavelength regions, such as UV and TIR for $\mathrm{O}_{3}$ or TIR and SWIR for CO, the vertical information content could be further increased but this has so far not been demonstrated for real data. For some special cases, measurements under different angles and polarization directions can also add height information. Highly resolved vertical profiles are only achievable with active methods (lidar) which are operating from space for aerosols but not yet for trace species. However, these techniques have very limited spatial coverage and therefore need to be combined with other measurements to provide the global picture.

Continuity of measurements is mainly determined by the availability of sensors and platforms. While currently several instruments dedicated to atmospheric composition measurements are in orbit, only a few of them have follow-up missions. For long-term observations, a high degree of consistency is needed and this can only be obtained from multi-instrument time series by cross-calibration of the results during several months of parallel operations as e.g. in the TOMS instrument series. Given the long preparation time for new missions, this is a major concern, in particular for high spatial resolution observations in the UV/visible.

The requirements for maintaining and improving the spaceborne observation system and filling the existing gaps can therefore be summarized as follows

- to provide continuity of observations by planning and deploying instruments before the end of the lifetime of existing sensors

- to add geostationary observations or a constellation of lowearth orbit measurements to improve spatial and temporal resolution and to sample the diurnal variation in chemistry

- to combine measurements from UV, visible and IR sensors for optimum species coverage and improved vertical resolution

- to develop novel multi-wavelength active systems to explore the vertical distribution of trace gases and aerosols from space

- to improve current instruments to be able to measure more reactive species such as hydrocarbons or reactive $\mathrm{N}$ species

- to make validation data sets available which facilitate validation of all the different satellite products, if possible under all relevant conditions (latitude, longitude, season, pollution level) and in near real time.

\subsubsection{Gaps in data management}

The adequacy of the existing observational networks for integration has been assessed by the IGACO report (IGACO Theme Team, 2004). The focus of the IGACO strategy, and of the WMO GAW programme charged with its implementation, is on operational systems providing continuity and reliability, and on setting priorities, in order to establish a technically and programmatically feasible long-term solution. The integration of more process-oriented observations 
derived from short-term campaigns certainly represents the most difficult challenge in the integration of observational data. Unique information on atmospheric composition, in particular data from very valuable research-based campaigns performed in the 1990s, is now lost to the scientific community due to the lack of integration into world data centres. Process-oriented campaigns are in fact a fundamental piece of the observing system in addition to the network observations designed for longer term studies. They often involve instrumentation that is not affordable for long-term studies, especially if mobile platforms (aircraft, zeppelin, mobile van, etc.) are involved. If such campaigns are performed simultaneously at different sites they can possibly be linked by models, further enhancing the value of an individual campaign.

An extremely promising technique that should be developed further is the so-called assimilation of data (see Section 4 above). Data assimilation should probably not be relied on for long-term trend detection of atmospheric composition changes since it involves numerical averaging that may cancel out detectable changes, but it is a very powerful tool to improve (short-term) predictions of atmospheric states. How does data assimilation help the cause of integration? By casting physical reality in a mathematical model and combining this with observations, many different types of observations can be utilized, and both the model and the observations can be challenged. This approach offers novels ways for satellite validation, but also supports the assessment of representativeness of point measurements (ground-based or airborne in situ) and total column or profile observations (ground-based remote sensing). It is extremely rare that operational measurements not specifically targeted for satellite validation observe the same volume of air at exactly the same time. Data assimilation offers a general way to compare in situ and remote sensing observations, i.e. to integrate information from various sources in a 4D (space and time) data space that can subsequently be interrogated to answer both science and policy relevant questions. To be meaningful, data assimilation requires a realistic mathematical description of the physical and chemical processes controlling atmospheric composition and transport, as well as adequate observations management.

\section{Conclusions: towards an integrated observing strategy}

A suitable observing system is a pre-requisite for informed decisions in a wide range of policy areas, including climate change and air quality, but also for health, international protocols and research requirements. Such an observation strategy therefore responds to political, societal and economic challenges and to the development of scientific knowledge. The challenges in designing an observation strategy for the future are therefore tremendous and range from technical issues like the instrumentation to be applied, how to treat data etc. to more political matters like funding and sustainability.

The IGACO report (IGACO Theme Team, 2004) has clearly set the stage for the implementation of future atmospheric observation strategies. The present paper has highlighted why observation strategies cannot only be policy-driven, and it has indicated the need for improving capacity for observation and analysis, and the need for linking research, long-term monitoring and operational programmes, bringing together the producers of global observations and the users that require them. In terms of atmospheric observations, the most difficult challenge will be to combine satellite, airborne and in situ observations in a way that adds more value to the whole than can be provided by any individual component.

Clearly, the diversity of technical and organisational structures and workflows implemented by different organizations and programmes continues to be a major obstacle for concerted approaches towards the implementation of a European or global integrated system for atmospheric observations. In particular, the fragmentation of efforts essentially decreases the efficiency in harmonizing the measurements and their quality, and in processing and providing data. To fully exploit the collective capacity of atmospheric observations, an overall strategy with improved coordination is needed. This will ensure a global level of standardization and interoperability in line with the requirements and recommendations of the GEOSS initiative (GEO 2005) and the priorities laid down in the GEO work plan for 2007-2009 (GEO 2007).

Resolving the increasingly more complex atmosphere and climate related science and policy issues is a major challenge, and funding for the long-term studies required in to this end is often inadequate. Still, EU initiatives performed within the capacities programme have been extremely important to provide the tools for research-oriented atmospheric chemistry network in the short (EUSAAR, EARLINET) and potentially long (IAGOS) term. Initiatives like the GEOmon project (Global Earth Observation and Monitoring of the atmosphere), building a prototype for a future integrated pan-European Atmospheric Observing System dealing with systematic observations of long-lived greenhouse gases, reactive gases, aerosols, and stratospheric ozone contributes to harmonizing the main European networks of surface and aircraft-based measurements of atmospheric composition parameters. These activities will, in turn, feed the GEMS project (Global and regional Earth-system (atmosphere) Monitoring using Satellite and in situ data) to create a system for operational global monitoring and medium and short-range forecasts of atmospheric chemistry and dynamics. Detecting atmospheric trends of key atmospheric compounds requires long ( $>10$ years) high quality records. The implementation of such a system is however jeopardized due to the limited long-term sustainability of the research based initiatives. The same consideration applies even more to other parts of the world. Long-term commitment of national and international funding agencies to maintain R\&D efforts and funding for atmospheric observations outside of policy-driven activities is of fundamental importance.

Clearly, the existing observation capacities should be maintained and improved. A cautious redistribution of the in situ observation effort in areas where density of information is more than sufficient could free up resources for areas where information is missing. Existing remote sensing platforms such as SCIAMACHY, MIPAS, IASI, GOME-2 or GOMOS instruments have been crucial to the characterization of atmospheric composition. Their limited lifetime and the extensive time required for their replacement implies that decisions about future observation capacities, in particular in Europe, must be taken rapidly. The global coverage of satellite missions and the need for additional in situ validation of satellite sensors highlights the need for a more adequate spatial coverage of in situ observations. As mentioned previously, the existing in situ observation capacities are mainly based on infrastructure operated at national level, mostly sustained in Europe by the Member States and resulting in an evidently inadequate distribution. Improving in situ measurement capacity for Air Quality and Climate studies outside of OECD countries will be a major challenge for the next decade. Development of autonomous samplers/analyzers, capacity building in Asia, Africa and Latin America, relocation of observation capacity for specific species (i.e. $\mathrm{O}_{3}$ ) will be among the priority actions. This should be sustained on the one hand by improving international cooperation in terms of cooperation between existing networks and data centres. On the other hand, it is important that OECD countries engage in providing financial and technical support to observation outside their political boundaries through international and bilateral agreements. There, a pan-state contribution to the observing effort would be extremely beneficial. It is a strong recommendation 
to environmental protection agencies to contributing long-term observation outside of their area of jurisdiction.

An integrated implementation of the IGACO recommendations needs to complement and reach beyond these existing projects and supply a true "system of systems" in the sense of GEOSS with integrated, dynamic and highly efficient data management. Improvements in global data management will also highlight and identify areas of deficiency regarding the techniques and coverage of observations themselves. A prerequisite for that is the use of international standards for the description of metadata as defined by ISO (ISO, 2003) and timely submission (where possible, in nearreal time) of observational data to international data archives. Global catalogues of metadata maintained by the WMO Information System (WIS) (http://wis.wmo.int/) will offer the possibility for efficient discovery and retrieval of data and will significantly improve our ability to make use of observational evidence to inform both science and policy.

\section{Acknowledgements}

This paper is a contribution from EU funded projects ACCENT, EARLINET and EUSAAR. We are thankful to Mariana Berthet and the ACCENT project office for their support in the preparation of the article.

\section{References}

Abbatt, J.P.D., Benz, S., Cziczo, D.J., Kanji, Z., Lohmann, U., Möhler, O., 2006. Solid ammonium sulfate aerosols as ice nuclei: a pathway for cirrus cloud formation. Science 313, 1770-1773.

Acker, K., Febo, A., Trick, S., Perrino, C., Bruno, P., Wiesen, P., Möller, D., Wieprecht, W., Auel, R., Giusto, M., Geyer, A., Platt, U., Allegrini, I., 2006. Nitrous acid in the urban area of Rome. Atmospheric Environment 40, 3123-3133.

Adams, K.L., Steele, P.T., Bogan, M.J., Sadler, N.M., Martin, S.I., Martin, A.N., Frank, M., 2008. Reagentless detection of Mycobacteria tuberculosis H37Ra in respiratory effluents in minutes. Analytical Chemistry 80, 5350-5357.

Afe, O.T., Richter, A., Sierk, B., Wittrock, F., Burrows, J.P., 2004. BrO emission from volcanoes: a survey using GOME and SCIAMACHY measurements. Geophysical Research Letters 31.

Aiken, A.C., DeCarlo, P.F., Jimenez, J.L., 2007. Elemental analysis of organic species with electron ionization high-resolution mass spectrometry. Analytical Chemistry $79,8350-8358$

Aiken, A.C., Decarlo, P.F., Kroll, J.H., Worsnop, D.R., Huffman, J.A., Docherty, K.S., Ulbrich, I.M., Mohr, C., Kimmel, J.R., Sueper, D., Sun, Y., Zhang, Q., Trimborn, A., Northway, M., Ziemann, P.J., Canagaratna, M.R., Onasch, T.B., Alfarra, M.R., Prevot, A.S.H., Dommen, J., Duplissy, J., Metzger, A., Baltensperger, U. Jimenez, J.L., 2008. O/C and OM/OC ratios of primary, secondary, and ambient organic aerosols with high-resolution time-of-flight aerosol mass spectrometry. Environmental Science and Technology 42, 4478-4485.

Albu, M., Barnes, I., Becker, K.H., Patroescu-Klotz, I., Mocanu, R., Benter, T., 2006. Rate coefficients for the gas-phase reaction of $\mathrm{OH}$ radicals with dimethyl sulfide: temperature and 02 partial pressure dependence. Physical Chemistry and Chemical Physics 8, 728-736.

Alfarra, M.R., Coe, H., Allan, J.D., Bower, K.N., Boudries, H., Canagaratna, M.R., Jimenez, J.L., Jayne, J.T., Garforth, A., Li, S., Worsnop, D.R., 2004. Characterization of urban and rural organic particulate in the Lower Fraser Valley using two aerodyne aerosol mass spectrometers. Atmospheric Environment 38, 5745-5758.

Alfarra, M.R., Paulsen, D., Gysel, M., Garforth, A.A., Dommen, J., Prévôt, A.S.H., Worsnop, D.R., Baltensperger, U., Coe, H., 2006. A mass spectrometric study of secondary organic aerosols formed from the photooxidation of anthropogenic and biogenic precursors in a reaction chamber. Atmospheric Chemistry and Physics 6, 5279-5293.

Allan, B., McFiggans, G., Plane, J., Coe, H., McFadyen, G., 2000. The nitrate radical in the remote marine boundary layer. Journal of Geophysical Research - Atmospheres. Journal of Geophysical Research 105, 24191-24204.

Allan, J.D., Jimenez, J.L., Williams, P.I., Alfarra, M.R., Bower, K.N., Jayne, J.T., Coe, H., Worsnop, D.R., 2003. Quantitative sampling using an Aerodyne aerosol mass spectrometer -1 . Techniques of data interpretation and error analysis. Journal of Geophysical Research - Atmospheres 108, 4090.

Allan, J.D., Coe, H., Bower, K.N., Alfarra, M.R., Delia, A.E., Jimenez, J.L., Middlebrook, A.M., Drewnick, F., Onasch, T.B., Canagaratna, M.R., Jayne, J.T. Worsnop, D.R., 2004. A generalised method for the extraction of chemically resolved mass spectra from Aerodyne aerosol mass spectrometer data. Journal of Aerosol Science 35, 909-922.

Allen, J.O., Steele, P., Gard, E.E., Hughes, L.S., Martin, S.I., Martin, A.N., Frank, M. 2000. Particle detection of Mycobacteria tuberculosis H37Ra in respiratory effluents in minutes. Analytical Chemistry 34, 211-217.
Amato, P., Ménager, M., Sancelme, M., Laj, P., Mailhot, G., Delort, A.-M., 2005. Microbial population in cloud water at the Puy de Dôme: implications for the chemistry of clouds. Atmospheric Environment 39, 4143-4153.

Amato, P., Demeer, F., Melaouhi, A., Fontanella, S., Martin-Biesse, A.-S., Sancelme, M. Laj, P., 2007. A fate for organic acids, formaldehyde and methanol in cloud water: their biotransformation by micro-organisms. Atmospheric Chemistry and Physics 7, 4159-4169.

Anderson, T.L., Charlson, R.J., Bellouin, N., Boucher, O., Chin, M., Christopher, S.A. Haywood, J., Kaufman, Y.J., Kinne, S., Ogren, J.A., Remer, L.A., Takemura, T., Tanré, D., Torres, O., Trepte, C.R., Wielicki, B.A., Winker, D.M., Yu, H., 2005. An "A-Train" strategy for quantifying direct climate forcing by anthropogenic aerosols. Bulletin of the American Meteorological Society 86, 1795-1809.

Ansmann, A., Riebesell, M., Weitkamp, C., 1990. Measurement of atmospheric aerosol extinction profiles with a Raman lidar. Optics Letters 15, 746-748.

Ansmann, A., Wandinger, U., Rille, O.L., Lajas, D., Straume, A.G., 2006. Particle backscatter and extinction profiling with the spaceborne high-spectral-resolution Doppler lidar ALADIN: methodology and simulations. Applied Optics 46 6606-6622.

Ansmann, A., Müller, D., 2005. Lidar and atmospheric aerosol particles. In: Weitkamp, C. (Ed.), Lidar. Range-resolved Optical Remote Sensing of the Atmosphere. Springer, New York, pp. 105-141.

Apel, E.C., Calvert, J.G., Gilpin, T.M., Parrish, D.D., Lonneman, W.A., 1999. The nonmethane hydrocarbon intercomparison experiment (NOMHICE): task 3. Journal of Geophysical Research 104, 26069-26086.

Apel, E.C., Calvert, J.G., Gilpin, T.M., Fehsenfeld, F.C., Lonneman, W.A., 2003. The nonmethane hydrocarbon intercomparison experiment (NOMHICE): task 4 ambient air. Journal of Geophysical Research 108, 4300

Apel, E.C., Hills, A.J., Lueb, R., Zindel, S., Eisele, S., Riemer, D.D., 2003. A fast-GC/MS system to measure C-2 to C-4 carbonyls and methanol aboard aircraft. Journal of Geophysical Research 108, 8794.

Apel, E.C., Brauers, T., Koppmann, R., Tillmann, R., Holzke, C., Wegener, R. Boßmeyer, J., Brunner, A., Ruuskanen, T., Jocher, M., Spirig, C., Steinbrecher, R., Meier, R., Steigner, D., Gómez Alvarez, E., Müller, K., Solomon, S.J., Schade, G. Young, D., Simmonds, P., Hopkins, J.R., Lewis, A.C., Legreid, G., Wisthaler, A., Hansel, A., Blake, R., Wyche, K., Ellis, A., Monks, P.S., 2008. Intercomparison of oxygenated volatile organic compound measurements at the SAPHIR atmosphere simulation chamber. Journal of Geophysical Research 113, D20307.

Archuleta, C.M., DeMott, P.J., Kreidenweis, S.M., 2005. Ice nucleation by surrogates for atmospheric mineral dust and mineral dust/sulfate particles at cirrus temperatures. Atmospheric Chemistry and Physics 5, 2617-2634.

Ardon, K., Levin, Z., Ganor, E., Klein, H., Bingemer, H., 2008. The effect of air pollution on ice nuclei concentration in Israel. In: International Conference on Clouds and Precipitation, Cancun, Mexico.

Arnott, W.P., Hamasha, K., Moosmüller, H., Sheridan, P.J., Ogren, J.A., 2005. Towards aerosol light-absorption measurements with a 7-wavelength aethalometer: evaluation with a photoacoustic instrument and 3-wavelength nephelometer. Aerosol Science and Technology 39, 17-29.

Asa-Awuku, A., Engelhart, G.J., Lee, B.H., Pandis, S.N., Nenes, A., 2009. Relating CCN activity, volatility, and droplet growth kinetics of $\beta$-caryophyllene secondary organic aerosol. Atmospheric Chemistry and Physics 9, 795-812.

Atkinson, R., Baulch, D.L., Cox, R.A., Crowley, J.N., Hampson, R.F., Hynes, R.G. Jenkin, M.E., Rossi, M.J., Troe, J., Wallington, T.J., 2008. Evaluated kinetic and photochemical data for atmospheric chemistry: volume IV - gas phase reactions of organic halogen species. Atmospheric Chemistry and Physics 8 , 4141-4496.

Aumann, H.H., Pagano, R.J., 1994. Atmospheric infrared sounder on the Earth observing system. Optical Engineering 33, 776-784

Ayers, J.D., Apodaca, R.L., Simpson, W.R., Baer, D.S., 2005. Off-axis cavity ringdown spectroscopy: application to atmospheric nitrate radical detection. Applied Optics 44, 7239-7242.

Bae, M.S., Schauer, J.J., DeMinter, J.T., Turner, J.R., Smith, D., Cary, R.A., 2004. Validation of a semi-continuous instrument for elemental carbon and organic carbon using a thermal-optical method. Atmospheric Environment 38, 2885-2893.

Bahreini, R., 2005. Measurements of secondary organic aerosol from oxidation of cycloalkenes, terpenes, and m-xylene using an Aerodyne aerosol mass spectrometer. Environmental Science and Technology 39, 5674-5688.

Baker, M.B., 1997. Cloud microphysics and climate. Science 276, 1072-1078.

Ball, S.M., Jones, R.L., 2003. Broad-band cavity ring-down spectroscopy. Chemical Reviews 103, 5239-5262.

Ball, S.M., Langridge, J.M., Jones, L., 2004. Broadband cavity enhanced absorption spectroscopy using light emitting diodes. Chemical Physics Letters 398 (1-3) 68-74.

Baltensperger, U., Streit, N., Weingartner, E., Nyeki, S., Prévôt, A.S.H., van Dingenen, R., Virkkula, A., Putaud, J.-P., Even, A., ten Brink, H., Blatter, A., Neftel, A., Gäggeler, H.W., 2002. Urban and rural aerosol characterization of summer smog events during the PIPAPO field campaign in Milan, Italy. Journal of Geophysical Research 107, 1-13.

Baltensperger, U., Kalberer, M., Dommen, J., Paulsen, D., Alfarra, M.R., Coe, H., Fisseha, R., Gascho, A., Gysel, M., Nyeki, S., Sax, M., Steinbacher, M. Prevot, A.S.H., Sjogren, S., Weingartner, E., Zenobi, R., 2005. Secondary organic aerosols from anthropogenic and biogenic precursors. Faraday Discussions 130 265-278.

Bao, H., 2006. Purifying synthetic barite for oxygen isotope measurement by dissolution and reprecipitation in a chelating solution. Analytical Chemistry 78, 304-309. 
Bao, H., Thiemens, M.H., 2000. Generation of $\mathrm{O}_{2}$ from $\mathrm{BaSO}_{4}$ using a $\mathrm{CO}_{2}$-laser fluorination system for simultaneous $\delta^{18} \mathrm{O}$ and $\delta^{17} \mathrm{O}$ analysis. Analytical Chemistry $72,4029-4032$.

Barret, B., 2003. Inversion et caractérisation de profils verticaux de constituants atmosphériques à partir de mesures FTIR sol. Ph.D. thesis in Chimie Quantique et Photophysique. Université Libre de Bruxelles, Brussels.

Barret, B., De Mazière, M., Demoulin, P., 2002. Retrieval and characterisation of ozone profiles from solar infrared spectra at the Jungfraujoch. Journal of Geophysical Research 107, 4788.

Barret, B., De Mazière, M., Mahieu, E., 2003. Ground-based FTIR measurements of CO from the Jungfraujoch: characterisation and comparison with in situ surface and MOPITT data. Atmospheric Chemistry and Physics 3, 2217-2223.

Bartenbach, S., Williams, J., Plass-Dülmer, C., Berresheim, H., Lelieveld, J., 2007. In situ measurement of reactive hydrocarbons at Hohenpeissenberg with comprehensive two-dimensional gas chromatography (GC $\times$ GC-FID): use in estimating $\mathrm{HO}$ and $\mathrm{NO}_{3}$. Atmospheric Chemistry and Physics 7, 1-14.

Baublys, K.A., Golding, S.D., Young, E., Kamber, B.S., 2004. Simultaneous determination of $\delta^{33} \mathrm{SV}$-CDT and $\delta^{34} \mathrm{SV}-\mathrm{CDT}$ using masses 48,49 and 50 on a continuous flow isotope ratio mass spectrometer. Rapid Communications in Mass Spectrometry $18,2765-2769$.

Baynard, T., Lovejoy, E.R., Pettersson, A., Brown, S.S., Lack, D., Osthoff, H., Massoli, P., Ciciora, S., Dube, W.P., Ravishankara, A.R., 2007. Design and application of a pulsed cavity ring-down aerosol extinction spectrometer for field measurements. Aerosol Science and Technology 41, 447-462.

Beaver, M.R., Garland, R.M., Hasenkopf, C.A., 2008. A laboratory investigation of the relative humidity dependence of light extinction by organic compounds from lignin combustion. Environmental Research Letters 3, 045003.

Beddows, D.C.S., Donovan, R.J., Harrison, R.M., Heal, M.R., Kinnersley, R.P., King, M.D., Nicholson, D.H., Thompson, K.C., 2004. Correlations in the chemica composition of rural background atmospheric aerosol in the UK determined in real time using time-of-flight mass spectrometry. Journal of Environmental Monitoring 6, 124-133.

Beer, R., Glavich, T.A., Rider, D.M., 2001. Tropospheric emission spectrometer for the Earth observing system's Aura satellite. Applied Optics 40, 2356-2367.

Beer, R., Shephard, M.W., Kulawik, S.S., Clough, S.A., Eldering, A., Bowman, K.W., Sander, S.P., Fisher, B.M., Payne, V.H., Luo, M.Z., Osterman, G.B., Worden, J.R. 2008. First satellite observations of lower tropospheric ammonia and methanol. Geophysical Research Letters 35.

Beirle, S., Platt, U., Wenig, M., Wagner, T., 2003. Weekly cycle of $\mathrm{NO}_{2}$ by GOME measurements: a signature of anthropogenic sources. Atmospheric Chemistry and Physics 3, 2225-2232.

Beirle, S., Platt, U., von Glasow, R., Wenig, M., Wagner, T., 2004. Estimate of nitrogen oxide emissions from shipping by satellite remote sensing. Geophysical Research Letters 31, L18102.

Beirle, S., Spichtinger, N., Stohl, A., Cummins, K.L., Turner, T., Boccippio, D. Cooper, O.R., Wenig, M., Grzegorski, M., Platt, U., Wagner, T., 2006. Estimating the $\mathrm{NO}_{\mathrm{x}}$ produced by lightning from GOME and NLDN data: a case study in the Gulf of Mexico. Atmospheric Chemistry and Physics 6, 1075-1089.

Beirle, S., Salzmann, M., Lawrence, M.G., Wagner, T., 2009. Sensitivity of satellite observations for freshly produced lightning $\mathrm{NO}_{\mathrm{x}}$. Atmospheric Chemistry and Physics 9, 1077-1094.

Bendle, J.A., Kawamura, K., Yamazaki, K., 2006. Seasonal changes in stable carbon isotopic composition of n-alkanes in the marine aerosols from the western North Pacific: implications for the source and atmospheric transport. Geochimica et Cosmochimica Acta 70, 13-26.

Benedetti, A., Morcrette, J.-J., Boucher, O., Dethof, A., Engelen, R.J., Fisher, M., Flentjes, H., Huneeus, N., Jones, L., Kaiser, J.W., Kinne, S., Mangold, A., Razinger, M., Simmons, A.J., Suttie, M., team, a.t.G.-A., 2009. Aerosol analysis and forecast in the ECMWF integrated forecast system: data assimilation. Journal of Geophysical Research 114, D13205.

Bernath, P.F., McElroy, C.T., Abrams, M.C., Boone, C.D., Butler, M., Camy-Peyret, C., Carleer, M., Clerbaux, C., Coheur, P.F., Colin, R., DeCola, P., DeMaziere, M. Drummond, J.R., Dufour, D., Evans, W.F.J., Fast, H., Fussen, D., Gilbert, K., Jennings, D.E., Llewellyn, E.J., Lowe, R.P., Mahieu, E., McConnell, J.C., McHugh, M., McLeod, S.D., Michaud, R., Midwinter, C., Nassar, R., Nichitiu, F, Nowlan, C., Rinsland, C.P., Rochon, Y.J., Rowlands, N., Semeniuk, K., Simon, P., Skelton, R., Sloan, J.J., Soucy, M.A., Strong, K., Tremblay, P., Turnbull, D. Walker, K.A., Walkty, I., Wardle, D.A., Wehrle, V., Zander, R., Zou, J., 2005. Atmospheric chemistry experiment (ACE): mission overview. Geophysical Research Letters 32, L15S01.

Birch, M.E., Cary, R.A., 1996. Elemental carbon-based method for monitoring occupational exposures to particulate diesel exhaust. Aerosol Science and Technology 25, 221-241.

Bissonnette, L.R., 1995. Multiple scattering of narrow light beams in aerosols Applied Physics B 60, 315-323.

Biswas, S., Fine, P.M., Geller, M.D., Hering, S.V., Sioutas, C., 2005. Performance evaluation of a recently developed water-based condensation particle counter Aerosol Science and Technology 39, 419-427.

Bitter, M., Ball, S.M., Povey, I.M., Jones, R.L., 2005. A broadband cavity ringdown spectrometer for in situ measurements of atmospheric trace gases. Atmospheric Chemistry and Physics 5, 2547-2560.

Blake, R.S., Whyte, C., Hughes, C.O., Ellis, A.M., Monks, P.S., 2004. Demonstration of proton-transfer reaction time-of-flight mass spectrometry for real-time analysis of trace volatile organic compounds. Analytical Chemistry 76, 3841-3845.
Blomquist, B.W., Huebert, B.J., Howell, S.G., Litchy, M.R., Twohy, C.H., Schanot, A., Baumgardner, D., Lafleur, B., Seebaugh, R., Laucks, M.L., 2001. An evaluation of the community aerosol inlet for the NCAR C-130 research aircraft. Journal of Atmospheric and Oceanic Technology 18, 1387-1397.

Böckmann, C., Miranova, D., Müller, D., Scheidenbach, L., Nessler, R., 2005. Microphysical aerosol parameters from multiwavelength lidar. Journal of the Optical Society of America 22, 518-528.

Boersma, K.F., Eskes, H.J., Meijer, E.W., Kelder, H.M., 2005. Estimates of lightning $\mathrm{NO}_{\mathrm{x}}$ production from GOME satellite observations. Atmospheric Chemistry and Physics 5, 2311-2331.

Böge, O., Miao, Y., Plewka, A., Herrmann, H., 2006. Formation of secondary organic particle phase compounds from isoprene gas-phase oxidation products: an aerosol chamber and field study. Atmospheric Environment 40, 2501-2509.

Bond, T.C., Anderson, T.L., Campbell, D., 1999. Calibration and intercomparison of filter-based measurements of visible light absorption by aerosols. Aerosol Science and Technology 30, 582-600.

Bortz, S.E., Prather, M.J., Cammas, J.-P., Thouret, V., Smit, H., 2006. Ozone, water vapor, and temperature in the upper tropical troposphere: variations over a decade of MOZAIC measurements. Journal of Geophysical Research 111, D05305.

Bossmeyer, J., Brauers, T., Richter, C., Rohrer, F., Wegener, R., Wahner, A., 2006. Simulation chamber studies on the $\mathrm{NO}_{3}$ chemistry of atmospheric aldehydes. Geophysical Research Letters 33, L18810.

Bovensmann, H., Burrows, J.P., Buchwitz, M., Frerick, J., Noel, S., Rozanov, V.V., Chance, K.V., Goede, A.P.H., 1999. SCIAMACHY: mission objectives and measurement modes. Journal of the Atmospheric Sciences 56, 127-150.

Brasseur, G.P., Prinn, R.G., Pszenny, A.A.P., 2003. Atmospheric Chemistry in a Changing World: an Integration and Synthesis of a Decade of Tropospheric Chemistry Research. Springer.

Brenninkmeijer, C.A.M., Crutzen, P., Boumard, F., Dauer, T., Dix, B., Ebinghaus, R., Filippi, D., Fischer, H., Franke, H., Friess, U., Heintzenberg, J., Helleis, F., Hermann, M., Kock, H.H., Koeppel, C., Lelieveld, J., Leuenberger, M., Martinsson, B.G., Miemczyk, S., Moret, H.P., Nguyen, H.N., Nyfeler, P., Oram, D., O'Sullivan, D., Penkett, S., Platt, U., Pupek, M., Ramonet, M., Randa, B., Reichelt, M., Rhee, T.S., Rohwer, J., Rosenfeld, K., Scharffe, D., Schlager, H., Schumann, U., Slemr, F., Sprung, D., Stock, P., Thaler, R., Valentino, F., van Velthoven, P., Waibel, A., Wandel, A., Waschitschek, K., Wiedensohler, A., Xueref-Remy, I., Zahn, A., Zech, U., Ziereis, H., 2007. Civil aircraft for the regular investigation of the atmosphere based on an instrumented container: the new CARIBIC system. Atmospheric Chemistry and Physics 7, 4953-4976.

Brinksma, E.J., Pinardi, G., Volten, H., Braak, R., Richter, A., Schonhardt, A., van Roozendael, M., Fayt, C., Hermans, C., Dirksen, R.J., Vlemmix, T., Berkhout, A.J.C., Swart, D.P.J., Oetjen, H., Wittrock, F., Wagner, T., Ibrahim, O.W., de Leeuw, G., Moerman, M., Curier, R.L., Celarier, E.A., Cede, A., Knap, W.H., Veefkind, J.P., Eskes, H.J., Allaart, M., Rothe, R., Piters, A.J.M., Levelt, P.F., 2008. The 2005 and 2006 DANDELIONS $\mathrm{NO}_{2}$ and aerosol intercomparison campaigns. Journal of Geophysical Research - Atmospheres 113.

Brioude, J., Cammas, J.-P., Cooper, O.R., Nedelec, P., 2008. Characterization of the composition, structure, and seasonal variation of the mixing layer above the extratropical tropopause as revealed by MOZAIC measurements. Journal of Geophysical Research 113, D00B01. doi:10.1029/2007JD009184

Brock, C.A., Schröder, F., Kärcher, B., Petzold, A., Busen, R., Fiebig, M., 2000. Ultrafine particle size distributions measured in aircraft exhaust plumes. Journal of Geophysical Research 105, 555-567.

Bronnimann, S., Buchmann, B., Wanner, H., 2002. Trends in near-surface ozone concentrations in Switzerland: the 1990s. Atmospheric Environment 36 , 2841-2852.

Brown, L.R., Farmer, C.B., Rinsland, C.P., Zander, R., 1992. Remote sensing of the atmosphere by high resolution infrared absorption spectroscopy. In: Rao, K.N. Weber, A. (Eds.), Spectroscopy of the Earth's Atmosphere and Interstellar Medium. Academic Press, San Diego, pp. 97-151.

Brown, S., Stark, H., Ciciora, S., Ravishankara, A., 2001. In situ measurement of atmospheric $\mathrm{NO}_{3}$ and $\mathrm{N}_{2} \mathrm{O}_{5}$ via cavity ring-down spectroscopy. Geophysical Research Letters 28, 3227-3230.

Brown, S.S., Stark, H., Ciciora, S.J., McLaughlin, R.J., Ravishankara, A.R., 2002. Simultaneous in situ detection of atmospheric $\mathrm{NO}_{3}$ and $\mathrm{N}_{2} \mathrm{O}_{5}$ via cavity ringdown spectroscopy. Review of Scientific Instruments 73, 3291-3301.

Brown, S.S., Stark, H., Ravishankara, A.R., 2002. Cavity ring-down spectroscopy for atmospheric trace gas detection: application to the nitrate radical $\left(\mathrm{NO}_{3}\right)$. Applied Physics B-Lasers and Optics 75, 173-182.

Brown, S.S., Stark, H., Ryerson, T.B., Williams, E.J., Nicks Jr., D.K., Trainer, M. Fehsenfeld, F.C., Ravishankara, A.R., 2003. Nitrogen oxides in the nocturnal boundary layer: simultaneous in situ measurements of $\mathrm{NO}_{3}, \mathrm{~N}_{2} \mathrm{O}_{5}, \mathrm{NO}_{2}$, $\mathrm{NO}$, and $\mathrm{O}_{3}$. Journal of Geophysical Research 108, 4299. doi:10.1029/2002JD002917.

Brown, S.S., Dube, W.P., Osthoff, H.D., Stutz, J., Ryerson, T.B., Wollny, A.G., Brock, C.A., Warneke, C., De Gouw, J.A., Atlas, E., Neuman, J.A., Holloway, J.S., Lerner, B.M., Williams, E.J., Kuster, W.C., Goldan, P.D., Angevine, W.M., Trainer, M., Fehsenfeld, F.C., Ravishankara, A.R., 2007. Vertical profiles in $\mathrm{NO}_{3}$ and $\mathrm{N}_{2} \mathrm{O}_{5}$ measured from an aircraft: results from the NOAA P-3 and surface platforms during the New England Air Quality Study 2004. Journal of Geophysical Research 112.

Brune, W.H., Stevens, P.S., Mather, J.H., 1995. Measuring $\mathrm{OH}$ and $\mathrm{HO}_{2}$ in the troposphere by laser-induced fluorescence at low pressure. Journal of Atmospheric Science 52.

Brunner, D., Staehelin, J., Maeder, J.A., Wohltmann, I., Bodeker, G.E., 2006. Variability and trends in total and vertically resolved stratospheric ozone based on the CATO ozone data set. Atmospheric Chemistry and Physics 6, 4985-5008. 
Brunner, A., Ammann, A., Neftel, A., Spirig, C., 2007. Methanol exchange between grassland and the atmosphere. Biogeosciences 4, 395-410.

Buchwitz, M., Khlystova, I., Bovensmann, H., Burrows, J.P., 2007. Three years of global carbon monoxide from SCIAMACHY: comparison with MOPITT and first results related to the detection of enhanced $\mathrm{CO}$ over cities. Atmospheric Chemistry and Physics 7, 2399-2411.

Bukowiecki, N., Kittelson, D.B., Watts, W.F., Burtscher, H., Weingartner, E., Baltensperger, U., 2002. Real-time characterization of ultrafine and accumulation mode particles in ambient combustion aerosols. Journal of Aerosol Science 33, 1139-1154.

Bulatov, V., Fisher, M., Schechter, I., 2002. Aerosol analysis by cavity-ring-down spectroscopy. Analytica Chimica Acta 466, 1-9.

Bulatov, V., Chen, Y.H., Khalmanov, A., Schechter, I., 2006. Absorption and scattering characterization of airborne microparticulates by a cavity ringdown technique. Analytical and Bioanalytical Chemistry 384, 155-160.

Bundke, U., Nillius, B., Jaenicke, R., Wetter, T., Klein, H., Bingemer, H., 2008. The fast ice nucleus chamber FINCH. Atmospheric Research 90 (2-4), 180-186.

Burrows, J.P., Weber, M., Buchwitz, M., Rozanov, V., Ladstatter-Weissenmayer, A., Richter, A., DeBeek, R., Hoogen, R., Bramstedt, K., Eichmann, K.U., Eisinger, M., 1999. The global ozone monitoring experiment (GOME): mission concept and first scientific results. Journal of the Atmospheric Sciences 56, 151-175.

Burtscher, H., Baltensperger, U., Bukowiecki, N., Cohn, P., Huglin, C., Mohr, M., Matter, U., Nyeki, S., Schmatloch, V., Streit, N., Weingartner, E., 2001. Separation of volatile and non-volatile aerosol fractions by thermodesorption: instrumental development and applications. Journal of Aerosol Science 32, 427-442.

Butkovskaya, N.I., Kukui, A., Pouvesle, N., Le Bras, G., 2005. Formation of nitric acid in the gas-phase $\mathrm{HO}_{2}+\mathrm{NO}$ reaction: effects of temperature and water vapor. Journal of Physical Chemistry - Atmospheres 109, 6509-6520.

Callies, J., Corpaccioli, E., Eisinger, M., Hahne, A., Lefebvre, A., 2000. GOME-2Metop's second-generation sensor for operational ozone monitoring. Esa Bulletin-European Space Agency, 28-36.

Cammas, J.-P., Brioude, J., Chaboureau, J.-P., Duron, J., Mari, C., Mascart, P., Nédélec, P., Smit, H., Pätz, H.-W., Volz-Thomas, A., Stohl, A., Fromm, M., 2009. Injection in the lower stratosphere of biomass fire emissions followed by longrange transport: a MOZAIC case study. Atmospheric Chemistry and Physics 9 , 5829-5846.

Cantrell, W., Robinson, C., 2006. Heterogeneous freezing of ammonium sulfate and sodium chloride solutions by long chain alcohols. Geophysical Research Letters 33, L07802.

Cape, J.N., Anderson, M., Rowland, A.P., Wilson, D., 2004. Organic nitrogen in precipitation across the United Kingdom. Water, Air, and Soil Pollution: Focus 4, 25-35.

Cappa, C.D., Lack, D.A., Burkholder, J.B., Ravishankara, A.R., 2008. Bias in filter-based aerosol light absorption measurements due to organic aerosol loading: evidence from laboratory measurements. Aerosol Science and Technology 42, 1022-1032.

Carn, S.A., Strow, L.L., de Souza-Machado, S., Edmonds, Y., Hannon, S., 2005. Quantifying tropospheric volcanic emissions with AIRS: the 2002 eruption of Mt. Etna (Italy). Geophysical Research Letters 32, L02301.

Carn, S.A., Krueger, A.J., Krotkov, N.A., Yang, K., Levelt, P.F., 2007. Sulfur dioxide emissions from Peruvian copper smelters detected by the ozone monitoring instrument. Geophysical Research Letters 34, L09801.

Carter, W.P.L., Cocker, D.R., Flitz, D.R., Malkina, I.L., Bummiller, K., Saver, C.G., Pisano, J.T., Bufalino, C., Song, C., 2005. A new environmental chamber for evaluation of gas-phase chemical mechanisms and secondary aerosol formation. Atmospheric Environment 39, 7768-7788.

Casciotti, K.L., Sigman, D.M., Hastings, M.G., Böhlke, J.K., Hilkert, A., 2002. Measurement of the oxygen isotopic composition of nitrate in seawater and freshwater using the denitrifier method. Analytical Chemistry 74, 4905-4912.

Cassanelli, P., Fox, D.J., Cox, R.A., 2007. Temperature dependence of pentyl nitrate formation from the reaction of pentyl peroxy radicals with NO. Physical Chemistry and Chemical Physics 9, 4332-4337.

Chahine, M.T., Pagano, T.S., Aumann, H.H., Atlas, R., Barnet, C., Blaisdell, J., Chen, L., Divakarla, M., Fetzer, E.J., Goldberg, M., Gautier, C., Granger, S., Hannon, S., Irion, F.W., Kakar, R., Kalnay, E., Lambrigtsen, B.H., Lee, S.Y., Le Marshall, J., McMillan, W.W., McMillin, L., Olsen, E.T., Revercomb, H., Rosenkranz, P., Smith, W.L., Staelin, D., Strow, L.L., Susskind, J., Tobin, D., Wolf, W., Zhou, L.H., 2006. Improving weather forecasting and providing new data on greenhouse gases. Bulletin of the American Meteorological Society 87, 911.

Chaikovsky, A., Balin, Y., Bukin, O., Chen, B., Ivanov, A., Plusnin, I., Tulinov, G., Zuev, V., 2008. Monitoring aerosol and ozone in cis regions: 3 years of CIS-LINET activity. In: Proceedings of the 24th International Laser Radar Conference, Boulder (CO), USA, 23-27 June.

Chan, M.N., Chan, C.K., 2007. Mass transfer effects on the hygroscopic growth of ammonium sulfate particles with a water-insoluble coating. Atmospheric Environment 41, 4423-4433.

Chang, C.C.Y., Silva, S.R., Kendall, C., Michalski, G., Casciotti, K.L., Wankel, S.D., 2004 Preparation and Analysis of Nitrogen-bearing Compounds in Water for Stable Isotope Ratio Measurement. In: Handbook of Stable Isotope Analytical Techniques, vol. 1. Elsevier Science, Amsterdam (Chapter 15).

Chattopadhyay, S., Ziemann, P.J., 2005. Vapor pressures of unsubstituted and substituted monocarboxylic and dicarboxylic acids measured using an improved thermal desorption particle beam mass spectrometry method. Aerosol Science and Technology 39, 1085-1100.

Chen, Y.L., DeMott, P.J., Kreidenweis, S.M., Rogers, D.C., Sherman, D.E., 2000. Ice formation by sulfate and sulfuric acid aerosol particles under upper-tropospheric conditions. Journal of Atmospheric Science 57, 3752-3766.
Choi, Chan, C., 2002. The effects of organic species on the hygroscopic behaviors of inorganic aerosols. Environmental Science and Technology 36, 2422-2428.

Choi, Y., Wang, Y.H., Zeng, T., Martin, R.V., Kurosu, T.P., Chance, K., 2005. Evidence of lightning $\mathrm{NO}_{\mathrm{x}}$ and convective transport of pollutants in satellite observations over North America. Geophysical Research Letters 32, L02805.

Chung, S.H., Seinfeld, J., 2002. Global distribution and climate forcing of carbonaceous aerosols. Journal of Geophysical Research 107.

Claeys, M., Graham, B., Vas, G., Wang, W., Vermeylen, R., Pashynska, V., Cafmeyer, J. Guyon, P., Andreae, M.O., Artaxo, P., Maenhaut, W., 2004. Formation of secondary organic aerosols through photooxidation of isoprene. Science 303. 1173-1176.

Clarisse, L., Coheur, P.F., Prata, A.J., Hurtmans, D., Razavi, A., Phulpin, T., HadjiLazaro, J. Clerbaux, C., 2008. Tracking and quantifying volcanic $\mathrm{SO}_{2}$ with IASI the September 2007 eruption at Jebel at Tai. Atmospheric Chemistry and Physics 8, 7723-7734.

Clarisse, L., Clerbaux, C., Dentener, F., Hurtmans, D., Razavi, A., Coheur, P.F., 2009 Global ammonia distribution derived from infrared satellite observations. Nature Geoscience 2, 479-483. doi:10.1038/ngeo551.

Clarmann, T.V., Glatthor, N., Koukouli, M.E., Stiller, G.P., Funke, B., Grabowski, U. Hoepfner, M., Kellmann, S., Linden, A., Milz, M., Steck, T., Fischer, H., 2007. MIPAS measurements of upper tropospheric $\mathrm{C}_{2} \mathrm{H}_{6}$ and $\mathrm{O}-3$ during the southern hemispheric biomass burning season in 2003. Atmospheric Chemistry and Physics 7, 5861-5872.

Clemitshaw, K.C., 2004. A review of instrumentation and measurement techniques for ground-based and airborne field studies of gas-phase tropospheric chemistry. Critical Reviews in Environmental Science and Technology 34, 1-108.

Clerbaux, C., Edwards, D.P., Deeter, M., Emmons, L., Lamarque, J.F., Tie, X.X. Massie, S.T., Gille, J., 2008. Carbon monoxide pollution from cities and urban areas observed by the Terra/MOPITT mission. Geophysical Research Letters 35 L03817. doi:10.1029/2007GL032300.

Clerbaux, C., Coheur, P.-F., Clarisse, L., Hadji-Lazaro, J., Hurtmans, D., Turquety, S., Bowman, K., Worden, H., Carn, S.A., 2008b. Measurements of $\mathrm{SO}_{2}$ profiles in volcanic plumes from the NASA Tropospheric Emission Spectrometer (TES). Geophysical Research Letters 35, L22807. doi:10.1029/2008GL035566.

Clerbaux C., Boynard, A., Clarisse, L., George, M., Hadji-Lazaro, J., Herbin, H., Hurtmans, D., Pommier, M., Razavi, A., Turquety, S., Wespes, C., Coheur, P.-F., 2009. Monitoring of atmospheric composition using the thermal infrared IASI/MetOp sounder. Atmospheric Chemistry and Physics 9, 6041-6054.

Cliff, S.S., Thiemens, M.H., 1994. High-precision isotopic determination of the ${ }^{18} \mathrm{O} /{ }^{16} \mathrm{O}$ and ${ }^{17} \mathrm{O} /{ }^{16} \mathrm{O}$ ratios in nitrous oxide. Analytical Chemistry 66, 2791-2793.

Cocker, D.R., Flagan, R.C., Seinfeld, J.H., 2001. State-of-the-art chamber facility for studying atmospheric aerosol chemistry. Environmental Science and Technology 35, 2594-2601.

Coe, H., Allan, J.D., 2006. Mass spectrometric methods for aerosol composition measurements. In: Heard, D.E. (Ed.), Analytical Techniques for Atmospheric Measurement. Blackwell Publishing Ltd., pp. 265-310.

Coheur, P.F., Barret, B., Turquety, S., Hurtmans, D., Hadji-Lazaro, J., Clerbaux, C., 2005. Retrieval and characterization of ozone vertical profiles from a thermal infrared nadir sounder. Journal of Geophysical Research - Atmospheres 110 (D24) D24303. doi:10.1029/2005JD005845.

Coheur, P.F., Herbin, H., Clerbaux, C., Hurtmans, D., Wespes, C., Carleer, M. Turquety, S., Rinsland, C.P., Remedios, J., Hauglustaine, D., Boone, C.D. Bernath, P.F., 2007. ACE-FTS observation of a young biomass burning plume: first reported measurements of $\mathrm{C}_{2} \mathrm{H}_{4}, \mathrm{C}_{3} \mathrm{H}_{6} \mathrm{O}, \mathrm{H}_{2} \mathrm{CO}$ and PAN by infrared occultation from space. Atmospheric Chemistry and Physics 7, 5437-5446.

Coheur, P.F., Clarisse, L., Turquety, S., Hurtmans, D., Clerbaux, C., 2009. IASI measurements of reactive trace species in biomass burning plumes. Atmospheric Chemistry and Physics 9, 5655-5667.

Collins, W.D., Rasch, P.J., Eaton, B.E., Khattatov, B.V., Lamarque, J.F., Zender, C.S., 2001. Simulating aerosols using a chemical transport model with assimilation of satellite aerosol retrievals: methodology for INDOEX. Journal of Geophysical Research - Atmospheres 106, 7313-7336.

Connolly, P.J., Flynn, M.J., Ulanowski, Z., Choularton, T.W., Gallagher, M.W. Bower, K.N., 2007. Calibration of 2-D imaging probes using calibration beads and ice crystal analogues. Part 1: the depth-of-field. Journal of Atmospheric and Oceanic Technology 24, 1860-1879.

Constapel, M., Schellentrager, M., Schmitz, O.J., Gäb, S., Brockmann, K.J., Giese, R. Benter, T., 2005. Atmospheric-pressure laser ionization: a novel ionization method for liquid chromatography/mass spectrometry. Rapid Communications in Mass Spectrometry 19, 326-336.

Cook, P.A., Savage, N.H., Turquety, S., Carver, G.D., O’Connor, F.M., Heckel, A., Stewart, D., Whalley, L.K., Parker, A.E., Schlager, H., Singh, H.B., Avery, M.A. Sachse, G.W., Brune, W., Richter, A., Burrows, J.P., Purvis, R., Lewis, A.C. Reeves, C.E., Monks, P.S., Levine, J.G., Pyle, J.A., 2007. Forest fire plumes over the North Atlantic: p-TOMCAT model simulations with aircraft and satellite measurements from the ITOP/ICARTT campaign. Journal of Geophysical Research - Atmospheres 112 .

Coplen, T.B., Böhlke, J.K., Casciotti, K.L., 2004. Using dual-bacterial denitrification to improve $\delta^{15} \mathrm{~N}$ determinations of nitrates containing mass-independent ${ }^{17} \mathrm{O}$. Rapid Communications in Mass Spectrometry 18, 245-250.

Cornell, S.E., Jickells, T.D., Cape, J.N., Rowland, A.P., Duce, R.A., 2003. Organic nitrogen deposition on land and coastal environments: a review of methods and data. Atmospheric Environment 37, 2173-2191.

Cotton, R.J., 2008. Results of ice nucleation ability of various aerosols sampled at the international workshop on comparing ice nucleation measuring systems (ICIS 
2007) using a continuous flow diffusion chamber. In: International Conference on Clouds and Precipitation, Cancun, Mexico.

Cozic, J., Mertes, S., Verheggen, B., Cziczo, D.J., Gallavardin, S.J., Walter, S., Baltensperger, U., Weingartner, E., 2008. Black carbon enrichment in atmospheric ice particle residuals observed in lower tropospheric mixed phase cloud. Journal of Geophysical Research 113. doi:10.1029/2007JD009266.

Crewell, S., Czekala, H., Löhnert, U., Simmer, C., Rose, T., Zimmermann, R., Zimmermann, R., 2001. Microwave radiometer for cloud carthography: a 22channel ground-based microwave radiometer for atmospheric research. Radio Science 36, 621-638.

Crosier, J., Allan, J.D., Coe, H., Bower, K.N., Formenti, P., Williams, P.I., 2007. Chemical composition of summertime aerosol in the Po Valley (Italy) Northern Adriatic and Black Sea. Quarterly Journal of the Royal Meteorological Society $133,61-75$

Crosier, J., Jimenez, J.L., Allan, J.D., Bower, K.N., Williams, P.I., Alfarra, M.R. Canagaratna, M.R., Jayne, J.T., Worsnop, D.R., Coe, H., 2007. Technical note: description and use of the new Jump mass spectrum mode of operation for the Aerodyne quadrupole aerosol mass spectrometers (Q-AMS). Aerosol Science and Technology 41, 865-872.

Cross, E.S., Slowik, J.G., Davidovits, P., Allan, J.D., Worsnop, D.R., Jayne, J.T Lewis, D.K., Canagaratna, M., Onasch, T.B., 2007. Laboratory and ambient particle density determinations using light scattering in conjunction with aerosol mass spectrometry. Aerosol Science and Technology 41, 343-359.

Dämmgen, U., Erisman, J.W., Cape, J.N., Grunhage, L., Fowler, D., 2005. Practical considerations for addressing uncertainties in monitoring bulk deposition. Environmental Pollution 134, 535-548.

Davis, 1980. Single aerosol-particle size and mass measurements using an electrodynamic balance. Journal of Colloid and Interface Science 75, 566-576.

Davis, 1990. The double-ring electrodynamic balance for microparticle characterization. Review of Scientific Instruments 61, 1281-1288.

Davison, B., Brunner, A., Ammann, A., Spirig, C., Jocher, M., Neftel, A., 2008. Cutinduced VOC emissions from agricultural grasslands. Plant Biology 10, 76-85.

Day, D.A., Wooldridge, P.J., Dillon, M.B., Thornton, J.A., Cohen, R.C., 2002. A therma dissociation laser-induced fluorescence instrument for in situ detection of $\mathrm{NO}_{2}$ peroxy nitrates, alkyl nitrates, and $\mathrm{HNO}_{3}$. Journal of Geophysical Research 107, 4046.

de Gouw, J.A., Warneke, C., Karl, T., Eerdekends, G., van der Veen, C., Fall, R., 2003. Sensitivity and specificity of atmospheric trace gas detection by proton-transfer-reaction mass spectrometry. International Journal of Mass Spectrometry 223-224, 365-382.

de Gouw, J.A., Warneke, C., 2007. Measurements of volatile organic compounds in the earths atmosphere using proton-transfer-reaction mass spectrometry. Mass Spectrometry Reviews 26, 223-257.

de Graaf, M., Stammes, P., 2005. SCIAMACHY absorbing aerosol index - calibration issues and global results from 2002-2004. Atmospheric Chemistry and Physics $5,2385-2394$.

de Laat, A.T.J., Gloudemans, A.M.S., Schrijver, H., van den Broek, M.M.P., Meirink, J.F. Aben, I., Krol, M., 2006. Quantitative analysis of SCIAMACHY carbon monoxide total column measurements. Geophysical Research Letters 33, L07807.

DeCarlo, P.F., 2004. Particle morphology and density characterization by combined mobility and aerodynamic diameter measurements. Part 1: theory. Aerosol Science and Technology 38, 1185-1205.

DeCarlo, P.F., Kimmel, J.R., Trimborn, A., Northway, M.J., Jayne, J.T., Aiken, A.C. Gonin, M., Fuhrer, K., Horvath, T., Docherty, K.S., Worsnop, D.R., Jimenez, J.L. 2006. Field-deployable, high-resolution, time-of-flight aerosol mass spectrometer. Analytical Chemistry 78, 8281-8289.

DeCarlo, P.F., Dunlea, E.J., Kimmel, J.R., Aiken, A.C., Sueper, D., Crounse, J. Wennberg, P.O., Emmons, L., Shinozuka, Y., Clarke, A., Zhou, J., Tomlinson, J., Collins, D.R., Knapp, D., Weinheimer, A.J., Montzka, D.D., Campos, T. Jimenez, J.L., 2008. Fast airborne aerosol size and chemistry measurements above Mexico City and Central Mexico during the MILAGRO campaign. Atmospheric Chemistry and Physics 8, 4027-4048.

Decesari, S., Facchini, M.C., Fuzzi, S., Tagliavini, E., 2000. Characterization of watersoluble organic compounds in atmospheric aerosols: a new approach. Journal of Geophysical Research 105, 1481-1489.

Decesari, S., Mircea, M., Cavalli, F., Fuzzi, S., Moretti, F., Tagliavini, E., Facchini, M.C., 2007. Source attribution of water-soluble organic aerosol by nuclear magnetic resonance spectroscopy. Environmental Science and Technology 41, 2479-2484.

DeMott, PJ., 2002. Laboratory studies of cirrus cloud processes. In: Lynch, D.K Sassen, K., Starr, D.O.C., Stephens, G. (Eds.), Cirrus. Oxford University Press, Oxford, pp. 102-135.

DeMott, P... Rogers, D.C. 1990. Freezing nucleation rates of dilute-solution droplets measured between $-30^{\circ}$ and $-40{ }^{\circ} \mathrm{C}$ in laboratory simulations of natura clouds. Journal of Atmospheric Science 47, 1056-1064.

Deschamps, P.Y., Bréon, F.M., Leroy, M., Podaire, A., Bricaud, A., Buriez, J.C., Seze, G. 1994. The POLDER mission: instrument characteristics and scientific objectives. IEEE Transactions on International Geoscience \& Remote Sensing 32 598-615.

Deuzé, J.L., Bréon, F.M., Devaux, C., Goloub, P., Herman, M., Lafrance, B., Maignan, F Marchand, A., Nadal, F., Perry, G., Tanré, D., 2001. Remote sensing of aerosols over land surfaces from POLDER-ADEOS-1 polarized measurements. Journal of Geophysical Research 106, 4913-4926.

Devolder, P., 2003. Atmospheric fate of small alkoxy radicals: recent experimenta and theoretical advances. Journal of Photochemistry and Photobiology A: Chemistry 157, 137-147.
Dhaniyala, S., Flagan, R., McKinney, K.A., Wenneberg, P.O., 2003. Novel aerosol/gas inlet for aircraft-based measurements. Aerosol Science and Technology 37, 828-840.

Dhaniyala, S., Wennberg, P.O., Flagan, R.C., Fahey, D.W., Northway, M.J., Gao, R.S., Bui, T.P., 2004. Stratospheric aerosol sampling: effect of a blunt-body housing on inlet sampling characteristics. Aerosol Science and Technology 38, 1080-1090.

DiCarlo, P. Brune, W.H., Martinez, M., Harder, H., Lescher, R., Ren, X., Thornberry, T. Carroll, M.A., Young, V., Shepson, P.B., Riemer, D., Apel, E., Campbell, C., 2004. Missing $\mathrm{OH}$ reactivity in a forest: evidence for unknown reactive biogenic VOCs. Science 304, 722-725.

Dillon, T.J., Horowitz, A., H, D., Crowley, J.N., Vereckner, L., Peeters, J., 2006. Reaction of $\mathrm{HO}$ with hydroxyacetone $\left(\mathrm{HOCH}_{2} \mathrm{C}(\mathrm{O}) \mathrm{CH}_{3}\right)$ : rate coefficients $(233-363 \mathrm{~K})$ and mechanism. Physical Chemistry and Chemical Physics 8, 236-246.

Dils, B., De Mazière, M., Müller, J.F., Blumenstock, T., Buchwitz, M., de Beek, R. Demoulin, P., Duchatelet, P., Fast, H., Frankenberg, C., Gloudemans, A., Griffith, D., Jones, N., Kerzenmacher, T., Kramer, I., Mahieu, E., Mellqvist, J., Mittermeier, R.L., Notholt, J., Rinsland, C.P., Schrijver, H., Smale, D., Strandberg, A., Straume, A.G., Stremme, W., Strong, K., Sussmann, R., Taylor, J., van den Broek, M., Velazco, V., Wagner, T., Warneke, T., Wiacek, A., Wood, S., 2006. Comparisons between SCIAMACHY scientific products and ground-based FTIR data for total columns of $\mathrm{CO}, \mathrm{CH}_{4}, \mathrm{CO}_{2}$ and $\mathrm{N}_{2} \mathrm{O}$. Atmospheric Chemistry and Physics 6, 1953-1976.

Dinar, E., Riziq, A.A., Spindler, C., Erlick, C., Kiss, G., Rudich, Y., 2008. The complex refractive index of atmospheric and model humic-like substances (HULIS) retrieved by a cavity ring down aerosol spectrometer (CRD-AS). Faraday Discussions 137, 279-295.

Diner, D., Becket, J., Reilly, T., Bruegge, C., Conel, J., Kahn, R., Martonchik, J., Ackermann, $T$, Davies, $R$, Gerstl, $S$, Gordon, $H$, Muller, J-P. Myneni, $R$, Sellers, P., Pinty, B., Verstraete, M., 1998. Multi-angle Imaging SpectroRadiometer (MISR) instrument description and experiment overview. IEEE Transactions on International Geoscience \& Remote Sensing 36, 1072-1087.

Ding, A.J., Wang, T., Thouret, V., Cammas, J.-P., Nédélec, P., 2008. Tropospheric ozone climatology over Beijing: analysis of aircraft data from the MOZAIC program. Atmospheric Chemistry and Physics 8, 1-13.

Divakarla, M., Barnet, C., Goldberg, M., Maddy, E, Irion, F, Newchurch, M. Liu, X.P. Wolf, W., Flynn, L., Labow, G., Xiong, X.Z., Wei, J., Zhou, L.H., 2008. Evaluation of atmospheric infrared sounder ozone profiles and total ozone retrievals with matched ozonesonde measurements, ECMWF ozone data, and ozone monitoring instrument retrievals. Journal of Geophysical Research - Atmospheres 113, D15308.

Donahue, N., Hartz, K., Chuong, B., Presto, A., Stanier, C., Rosenhørn, T., Robinson, A., Pandis, S., 2005. Critical factors determining the variation in SOA yields from terpene ozonolysis: a combined experimental and computational study. Faraday Discussions 130, 295-309.

Donahue, N.M., Robinson, A.L., Stainer, C.O., Pandis, S.N., 2006. Coupled partitioning, 30 dilution, and chemical aging of semivolatile organics. Environmental Science and Technology 40, 2635-2643.

Donovan, D.P., van Lammeren, A.C.A.P., 2001. Cloud effective particle size and water content profile retrievals using combined lidar and radar observations - 1 . Theory and examples. Journal of Geophysical Research 106, 27425-27448.

Drewnick, F., Schwab, J.J., Hogrefe, O., Peters, S., Husain, L., Diamond, D., Weber, R., Demerjian, K.L., 2003. Intercomparison and evaluation of four semi-continuous PM2.5 sulfate instruments. Atmospheric Environment 37, 3335-3350.

Drewnick, F., Hings, S.S., DeCarlo, P., Jayne, J.T., Gonin, M., Fuhrer, K., Weimer, S., Jimenez, J.L., Demerjian, K.L., Borrmann, S., Worsnop, D.R., 2005. A new time-offlight aerosol mass spectrometer (TOF-AMS)-instrument description and first field deployment. Aerosol Science and Technology 39, 637-658.

Drewnick, F., Schneider, J., Hings, S.S., Hock, N., Noone, K., Targino, A., Weimer, S., Borrmann, S., 2007. Measurement of ambient, interstitial, and residual aerosol particles on a mountaintop site in central Sweden using an aerosol mass spectrometer and a CVI. Journal of Atmospheric Chemistry 56, 1-20.

Drummond, J.R., Mand, G.S., 1996. The measurements of pollution in the troposphere (MOPITT) instrument: overall performance and calibration requirements. Journal of Atmospheric and Oceanic Technology 13, 314-320.

Duarte, R.M.B.O., Santos, E.B.H., Pio, C.A., Duarte, A.C., 2007. Comparison of structural features of water-soluble organic compounds from atmospheric aerosols with those of aquatic humic substances. Atmospheric Environment 41, 81008113.

Dubé, W.P., Brown, S.S., Osthoff, H.D., 2006. Aircraft instrument for simultaneous, in situ measurement of $\mathrm{NO}_{3}$ and $\mathrm{N}_{2} \mathrm{O}_{5}$ via pulsed cavity ring-down spectroscopy. Review of Scientific Instruments 77, 034101-034111.

Dubovik, O., Holben, B.N., Eck, T.F., Smirnov, A., Kaufman, Y.J., King, M.D., Tanré, D., Slutsker, I., 2002. Variability of absorption and optical properties of key aerosol types observed in worldwide locations. Journal of Atmospheric Science 59, 590-608.

Dubovik, O., Lapyonok, T., Kaufman, Y.J., Chin, M., Ginoux, P., Kahn, R.A., Sinyuk, A., 2008. Retrieving global aerosol sources from satellites using inverse modeling. Atmospheric Chemistry and Physics 8, 209-250.

Dubovik, O., King, M.D., 2000. A flexible inversion algorithm for retrieval of aerosol optical properties from sun and sky radiance measurements. Journal of Geophysical Research 105, 20673-20696.

Dufour, E., Breon, F.M., Peylin, P., 2004. $\mathrm{CO}_{2}$ column averaged mixing ratio from inversion of ground-based solar spectra. Journal of Geophysical Research 109, D09304.

Dufour, G., Boone, C.D., Rinsland, C.P., Bernath, P.F., 2006. First space-borne measurements of methanol inside aged southern tropical to mid-latitude 
biomass burning plumes using the ACE-FTS instrument. Atmospheric Chemistry and Physics 6, 3463-3470.

Dufour, G., Szopa, S., Hauglustaine, D.A., Boone, C.D., Rinsland, C.P., Bernath, P.F. 2007. The influence of biogenic emissions on upper-tropospheric methanol as revealed from space. Atmospheric Chemistry and Physics 7, 6119-6129.

Duft, Leisner, T., 2004. Laboratory evidence for volume-dominated nucleation of ice in supercooled water microdroplets. ACP 4, 1997-2000.

Duplissy, J., Gysel, M., Alfarra, M.R., Dommen, J., Metzger, A., Prevot, A.S.H., Weingartner, E., Laaksonen, A., Raatikainen, T., Good, N., Turner, S.F. McFiggans, G., Baltensperger, U., 2008. Cloud forming potential of secondary organic aerosol under near atmospheric conditions. Geophysical Research Letters 35, L03818.

Duplissy, J., Gysel, M., Sjogren, S., Meyer, N., Good, N., Kammermann, L., Michaud, V. Weigel, R., Martins dos Santos, S., Gruening, C., Villani, P., Laj, P., Sellegri, K., Metzger, A., McFiggans, G., Wehrle, G., Richter, R., Dommen, J., Ristovski, Z., Baltensperger, U., Weingartner, E., 2009. Intercomparison study of six HTDMAs: results and general recommendations for HTDMA operation. Atmospheric Measurement Techniques 2, 363-378.

Durant, A.J., Shaw, R.A., 2005. Evaporation freezing by contact nucleation inside-out. Geophysical Research Letters 32, L20814

Dusek, U., Frank, G.P., Hildebrandt, L., Curtius, J., Schneider, J., Walter, S., Chand, D., Drewnick, F., Hings, S., Jung, D., Borrmann, S., Andreae, M.O., 2006. Size matters more than chemistry for cloud-nucleating ability of aerosol particles. Science 312, 1375-1378.

Dymarska, M., Murray, B.J., Sun, L.M., Eastwood, M.L., Knopf, D.A., Bertram, A.K., 2006. Deposition ice nucleation on soot at temperatures relevant for the lower troposphere. Journal of Geophysical Research 111, D04204.

EANET (Acid Deposition Monitoring Network in East Asia), 2000. Quality Assurance/Quality Control (QA/QC) Program for Wet Deposition Monitoring in East Asia. The Second Interim Scientific Advisory Group Meeting of Acid Deposition Monitoring Network in East Asia.

Eckhardt, S., Prata, A.J., Seibert, P., Stebel, K., Stohl, A., 2008. Estimation of the vertical profile of sulfur dioxide injection into the atmosphere by a volcanic eruption using satellite column measurements and inverse transport modeling. Atmospheric Chemistry and Physics 8, 3881-3897.

Eddy, P., Natarajan, A., Dhaniyala, S., 2006. Subisokinetic sampling characteristics of high speed aircraft inlets: a new CFD-based correlation considering inlet geometries. Journal of Aerosol Science 37, 1853-1870.

Edwards, G.D., Cantrell, C.A., Stephens, S., Hill, B., Goyea, O., Shetter, R.E., Mauldin, R.L., Kosciuch, E., Tanner, D.J., Eisele, F.L., 2003. Chemical ionization mass spectrometer instrument for the measurement of tropospheric $\mathrm{HO}_{2}$ and $\mathrm{RO}_{2}$. Analytical Chemistry 75, 5317-5327.

Edwards, D.P., Emmons, L.K., Hauglustaine, D.A., Chu, D.A., Gille, J.C., Kaufman, Y.J., Petron, G., Yurganov, L.N., Giglio, L., Deeter, M.N., Yudin, V., Ziskin, D.C., Warner, J., Lamarque, J.F., Francis, G.L., Ho, S.P., Mao, D., Chen, J., Grechko, E.I., Drummond, J.R., 2004. Observations of carbon monoxide and aerosols from the Terra satellite: Northern Hemisphere variability. Journal of Geophysical Research - Atmospheres 109, D24202.

Edwards, D.P., Emmons, L.K., Gille, J.C., Chu, A., Attie, J.L., Giglio, L., Wood, S.W., Haywood, J., Deeter, M.N., Massie, S.T., Ziskin, D.C., Drummond, J.R., 2006 Satellite-observed pollution from Southern Hemisphere biomass burning. Journal of Geophysical Research - Atmospheres 111, D14312.

Eisinger, M., Burrows, J.P., 1998. Tropospheric sulfur dioxide observed by the ERS-2 GOME instrument. Geophysical Research Letters 25, 4177-4180.

Elbern, H., Schmidt, H., Ebel, A., 1997. Variational data assimilation for tropospheric chemistry modeling. Journal of Geophysical Research 102, 15967-15985.

Elbern, H., Strunk, A., Schmidt, H., Talagrand, O., 2007. Emission rate and chemical state estimation by 4-dimensional variational inversion. Atmospheric Chemistry and Physics 7, 3749-3769.

Erdmann, N., Dell'Acqua, A., Cavalli, P., Gruning, C., Omenetto, N., Putaud, J.P., Raes, F., Van Dingenen, R., 2005. Instrument characterization and first application of the single particle analysis and sizing system (SPASS) for atmospheric aerosols. Aerosol Science and Technology 39, 377-393.

Eremenko, M., Dufour, G., Foret, G., Keim, C., Orphal, J., Beekmann, M., Bergametti, G., Flaud, J.M., 2008. Tropospheric ozone distributions over Europe during the heat wave in July 2007 observed from infrared nadir spectra recorded by IASI. Geophysical Research Letters 35, L18805.

Erisman, J.W., Mols, H., Fonteijn, P., Geusebroek, M., Draaijers, G., Bleeker, A., van der Veen, D., 2003. Field intercomparison of precipitation measurements performed within the framework of the Pan European Intensive Monitoring Program of EU/ICP forest. Environmental Pollution 125, 139-155.

Erisman, J.W., Hensen, A., Mosquera, J., Sutton, M., Fowler, D., 2005. Deposition monitoring networks: what monitoring is required to give reasonable estimates of ammonia/ammonium? Environmental Pollution 135, 419-431.

European Space Agency (ESA), 2004. Earth Clouds, Aerosols, and Radiation Explorer. ESA SP-1279(1), Technical report. ESTEC, Noorwijk, The Netherlands.

Esselborn, M., Wirth, M., Fix, A., Tesche, M., Ehret, G., 2008. Airborne high spectral resolution lidar for measuring aerosol extinction and backscatter coefficients. Applied Optics 47, 346-358.

Esselborn, M., Wirth, M., Fix, A., Weinzierl, B., Rasp, K., Tesche, M., Petzold, A. Spatial distribution and optical properties of Saharan dust observed by airborne high spectral resolution lidar during SAMUM 2006. Tellus B 61, 131-143.

Fagerli, H., Aas, W., 2008. Trends of nitrogen in air and precipitation: model results and observations at EMEP sites in Europe, 1980-2003. Environmental Pollution $154,448-461$.
Fan, J., Zhang, R., 2004. Atmospheric oxidation mechanism of isoprene. Environmental Chemistry 1, 140-149.

Fang, J., Kawamura, K., Ishimura, Y., Matsumoto, K., 2002. Carbon isotopic composition of fatty acids in the marine aerosols from the western North Pacific: implication for the source and atmospheric transport. Environmental Science and Technology 36, 2598-2604.

Feingold, G., Furrer, R., Pilewskie, P., Remer, L.A., Min, Q., Jonsson, H., 2006. Aerosol indirect effect studies at Southern Great Plains during the May 2003 intensive operations period. Journal of Geophysical Research 111, D05S14.

Feingold, G., Grund, C.J., 1994. Feasibility of using multiwavelength lidar measurements to measure cloud condensation nuclei. Journal of Atmospheric and Oceanic Technology 11, 1543-1558.

Fergenson, D.P., Pitesky, M.E., Tobias, H.J., Steele, P.T., Czerwieniec, G.A., Russell, S.C., Lebrilla, C.B., Horn, J.M., Coffee, K.R., Srivastava, A., Pillai, S.P., Shih, M.T.P., Hall, H.L., Ramponi, A.J., Chang, J.T., Langlois, R.G., Estacio, P.L., Hadley, R.T. Frank, M., Gard, E.E., 2004. Reagentless detection and classification of individual bioaerosol particles in seconds. Analytical Chemistry 76, 373-378.

Field, P.R., Möhler, O., Connolly, P., Krämer, M., Cotton, R., Heymsfield, A.J., Saathoff, H., Schnaiter, M., 2006. Some ice nucleation characteristics of Asian and Saharan desert dust. Atmospheric Chemistry and Physics 6, 2991-3006.

Fietkau, S., Medeke, T., Richter, A., Sheode, N., Sinnhuber, B.-M., Wittrock, F. Theys, N., van Roozendael, M., Burrows, J.P., 2007. Ground-based measurements of tropospheric and stratospheric bromine monoxide above Nairobi $\left(1^{\circ} \mathrm{S}, 36^{\circ} \mathrm{E}\right)$. Atmospheric Chemistry and Physics Discussions 7, 6527-6555.

Fioletov, V.E., Bodeker, G.E., Miller, A.J., McPeters, R.D., Stolarski, R., 2002. Global and zonal total ozone variations estimated from ground-based and satellite measurements: 1964-2000. Journal of Geophysical Research - Atmospheres $107,4647$.

Fischer, H., Birk, M., Blom, C., Carli, B., Carlotti, M., von Clarmann, T., Delbouille, L., Dudhia, A., Ehhalt, D., Endemann, M., Flaud, J.M., Gessner, R., Kleinert, A., Koopman, R., Langen, J., Lopez-Puertas, M., Mosner, P., Nett, H., Oelhaf, H., Perron, G., Remedios, J., Ridolfi, M., Stiller, G., Zander, R., 2008. MIPAS: an instrument for atmospheric and climate research. Atmospheric Chemistry and Physics 8, 2151-2188.

Fishman, J., Brackett, V.G., 1997. The climatological distribution of tropospheric ozone derived from satellite measurements using version 7 total ozone mapping spectrometer and stratospheric aerosol and gas experiment data sets. Journal of Geophysical Research - Atmospheres 102, 19275-19278.

Fisseha, R., Dommen, J., Sax, M., Paulsen, D., Kalberer, M., Maurer, R., Höfler, F., Weingartner, E., Baltensperger, U., 2004. Identification of organic acids in secondary organic aerosol and the corresponding gas phase from chamber experiments. Analytical Chemistry 76, 6535-6540.

Fisseha, R., Saurer, M., Jäggi, M., Szidat, S., Siegwolf, R.T.W., Baltensperger, U., 2006. Determination of stable carbon isotopes of organic acids and carbonaceous aerosols in the atmosphere. Rapid Communications in Mass Spectrometry 20, 2343-2347.

Fisseha, R., Saurer, M., Jäggi, M., Szidat, S., Samburova, V., Baltensperger, U., 2009. Characterization of primary and secondary sources of organic acids and carbonaceous aerosols using stable carbon isotopes. Atmospheric Environment 43, 431-437.

Forster, P., Van Dorland, R., Schulz, M., Raga, G., Prinn, R., Nganga, J., Myhre, G., Lowe, D.C., Lean, J., Haywood, J., Betts, R., Fahey, D.W., Ramaswamy, V., Artaxo, P., Bernsten, T., 2007. Changes in atmospheric constituents and in radiative forcing. In: Climate Change 2007: the Physical Science Basis. Contribution of Working Group I to the Fourth Assessment Report of the Intergovernmental Panel on Climate Change. Publisher Cambridge University Press, Cambridge, UK and New York, NY, USA.

Fowler, D., Pilegaard, K., Sutton, M.A., Ambus, P., Raivonen, M., Duyzer, J., Simpson, D., Fagerli, H., Schjoerring, J.K., Neftel, A., Burkhardt, J., Daemmgen, U., Neirynck, J., Personne, E., Wichink-Kruit, R., Butterbach-Bahl, K., Flechard, C., Tuovinen, J.P., Coyle, M., Fuzzi, S., Gerosa, G., Granier, C., Loubet, B., Altimir, N., Gruenhage, L., Ammann, C., Cieslik, S., Paoletti, E., Mikkelsen, T.N., Ro-Poulsen, H., Cellier, P. Cape, J.N., Isaksen, I.S.A., Horváth, L., Loreto, F., Niinemets, Ü., Palmer, P.I., Rinne J., Laj, P., Maione, M., Misztal, P., Monks, P., Nemitz, E., Nilsson, D., Pryor, S. Gallagher, M.W., Vesala, T., Skiba, U., Brüeggemann, N., Zechmeister-Boltenstern, S., Williams, J., O'Dowd, C., Facchini, M.C., de Leeuw, G., Flossman, A Chaumerliac, N., Erisman, J.W. Atmospheric composition change: ecosystems Atmosphere interactions, in this issue.

Franke, K., Richter, A., Bovensmann, H., Eyring, V., Jöckel, P., Burrows, J.P., 2008. Ship emitted $\mathrm{NO}_{2}$ in the Indian Ocean: comparison of model results with satellite data. Atmospheric Chemistry and Physics Discussions 8, 15997-16025.

Frankenberg, C., Meirink, J.F., van Weele, M., Platt, U., Wagner, T., 2005. Assessing methane emissions from global space-borne observations. Science 308, 1010 1014.

Frankenberg, C., Bergamaschi, P., Butz, A., Houweling, S., Meirink, J.F., Notholt, J. Petersen, A.K. Schrijver, H., Warneke, T., Aben, I., 2008. Tropical methane emissions: a revised view from SCIAMACHY onboard ENVISAT. Geophysical Research Letters 35, L15811.

Fraser, R.S., Kaufman, Y.J., Mahoney, R.L., 1984. Satellite measurements of aerosol mass and transport. Atmospheric Environment 18, 2577-2584.

Freudenthaler, V., Esselborn, M., Wiegner, M., Heese, B., Tesche, M., Ansmann, A., Müller, D., Althausen, D., Wirth, M., Fix, A., Ehret, G., Knippertz, P., Toledano, C. Gasteiger, J., Garhammer, M., Seefeldner, M., 2009. Depolarization-ratio profiling at several wavelengths in pure Saharan dust during SAMUM 2006. Tellus 61 B, 165-179. 
Frieß, U., Wagner, T., Pundt, I., Pfeilsticker, K., Platt, U., 2001. Spectroscopic measurements of tropospheric iodine oxide at Neumayer station, Antarctica. Geophysical Research Letters 28, 1941-1944.

Frieß, U., Hollwedel, J., König-Langlo, G., Wagner, T., Platt, U., 2004. Dynamics and chemistry of tropospheric bromine explosion events in the Antarctic coastal region. Journal of Geophysical Research 1009, D06305.

Frieß, U., Monks, P.S., Remedios, J.J., Rozanov, A., Sinreich, R., Wagner, T., Platt, U. 2006. MAX-DOAS O_4 measurements: a new technique to derive information on atmospheric aerosols: 2. Modeling studies. Journal of Geophysical Research 111, D14204.

Fuentes, M., Chaudhuri, A., Holland, D.M., 2007. Bayesian entropy for spatial sampling design of environmental data. Environmental and Ecological Statistics $14,323-340$

Fuzzi, S., Decesari, S., Facchini, M.C., Matta, E., Mircea, M., Tagliavini, E., 2001. A simplified model of the water soluble organic component of atmospheric aerosols. Geophysical Research Letters 28, 4079-4082.

Fuzzi, S., Andreae, M.O., Huebert, B.J., Kulmala, M., Bond, T.C., Boy, M., Doherty, S.J., Guenther, A., Kanakidou, M., Kawamura, K., Kerminen, V.M., Lohmann, U. Russell, L.M., Pöschl, U., 2006. Critical assessment of the current state of scientific knowledge, terminology, and research needs concerning the role of organic aerosols in the atmosphere, climate, and global change. Atmospheric Chemistry and Physics 6, 2017-2038.

Gallar, C., Brock, C.A., Jimenez, J.L., Simons, C., 2006. A variable supersaturation condensation particle sizer. Aerosol Science and Technology 40, 431-436.

Gamero-Castaño, M., De la Mora, J.F., 2000. A condensation nucleus counter (CNC) sensitive to singly charged sub-nanometer particles. Journal of Aerosol Science 31, 757-772.

Gard, E., Mayer, J.E., Morrical, B.D., Dienes, T., Fergenson, D.P., Prather, K.A., 1997. Real-time analysis of individual atmospheric aerosol particles: design and performance of a portable ATOFMS. Analytical Chemistry 69, 4083-4091.

Garland, R.M., Ravishankara, A.R., Lovejoy, E.R., Tolbert, M.A., Baynard, T., 2007. Parameterization for the relative humidity dependence of light extinction: organic-ammonium sulfate aerosol. Journal of Geophysics Research 112, D19303.

Gaussiat, N., Sauvageot, H., Illingworth, A.J., 2003. Cloud liquid water and ice content retrieval by multi-wavelength radar. Journal of Atmospheric and Oceanic Technology 20, 1264-1275.

Gelencsér, A., May, B., Simpson, D., Sanchez-Ochoa, A., Kasper-Giebl, A. Puxbaum, H., Caseiro, A., Pio, C., Legrand, M., 2007. Source apportionment of PM2.5 organic aerosol over Europe: primary/secondary, natural/anthropogenic, fossil/biogenic origin. Journal of Geophysical Research 112, D23S04. doi:10.1029/2006JD008094

Generoso, S., Breon, F.M., Chevallier, F., Balkanski, Y., Schulz, M., Bey, I., 2007. Assimilation of POLDER aerosol optical thickness into the LMDz-INCA model: implications for the Arctic aerosol burden. Journal of Geophysical Research 112 , D02311.

Gerbig, C., Schmitgen, S., Kley, D., Volz-Thomas, A., 1999. An improved fast-response vacuum-UV resonance fluorescence $\mathrm{CO}$ instrument. Journal of Geophysical Research 104, 1699-1704.

Giles, J., 2005. Climate science: the dustiest place on Earth. Nature 434, 816-819.

Glasius, M., Lahaniati, M., Calogirou, A., Di Bella, D., Jensen, N.R., Hjorth, J. Kotzias, D., Larsen, B.R., 2000. Carboxylic acids in secondary aerosols from oxidation of cyclic monoterpenes by ozone. Environmental Science and Technology 34, 1001-1010.

Glatthor, N., von Clarmann, T., Fischer, H., Funke, B., Grabowski, U., Hopfner, M. Kellmann, S., Linden, A., Milz, M., Steck, T., Stiller, G.P., 2007. Global peroxyacetyl nitrate (PAN) retrieval in the upper troposphere from limb emission spectra of the Michelson Interferometer for Passive Atmospheric Sounding (MIPAS). Atmospheric Chemistry and Physics 7, 2775-2787.

Goldan, P.D., Kuster, W.C., Williams, E., Murphy, P.C., Fehsenfeld, F.C., Meagher, J. 2004. Nonmethane hydrocarbon and oxy hydrocarbon measurements during the 2002 New England Air Quality Study. Journal of Geophysical Research 109, D21.

Goldstein, A.H., Shaw, S.L., 2003. Isotopes of volatile organic compounds: an emerging approach for studying atmospheric budgets and chemistry. Chemical Reviews 103, 5025-5048.

Graus, M., Müller, M., Wisthaler, A., Hansel, A., 2007. High resolution PTR-TOFMS performance assessment and applicability in atmospheric sciences. Geophysical Research Abstracts 9, 10543.

Gros, V., Williams, J., Lawrence, M.G., von Kuhlmann, R., van Aardenne, J., Atlas, E. Chuck, A., Edwards, D.P., Stroud, V., Krol, M., 2004. Tracing the origin and ages of interlaced atmospheric pollution events over the tropical Atlantic Ocean with in situ measurements, satellites, trajectories, emission inventories, and global models. Journal of Geophysical Research - Atmospheres 109.

Gross, D.S., Galli, M.E., Kalberer, M., Prevot, A.S.H., Dommen, J., Alfarra, M.R Duplissy, J. Gaeggeler, K., Gascho, A., Metzger, A., Baltensperger, U., 2006. Realtime measurement of oligomeric species in secondary organic aerosol with the aerosol time-of-flight mass spectrometer. Analytical Chemistry 78, 2130-2137.

Guazzotti, S.A., Suess, D.T., Coffee, K.R., Quinn, P.K., Bates, T.S., Wisthaler, A Hansel, A., Ball, W.P., Dickerson, R.R., Neususs, C., Crutzen, P.J., Prather, K.A 2003. Characterization of carbonaceous aerosols outflow from India and Arabia: biomass/biofuel burning and fossil fuel combustion. Journal of Geophysical Research - Atmospheres 108, 4485.

Guenther, A., Karl, T., Harley, P., Wiedinmyer, C., Palmer, P.I., Geron, C., 2006. Estimates of global terrestrial isoprene emissions using MEGAN (Model of
Emissions of Gases and Aerosols from Nature). Atmospheric Chemistry and Physics 6, 3181-3210.

Gysel, M., McFiggans, G.B., Coe, H., 2009. Inversion of tandem differential mobility analyser (TDMA) measurements. Journal of Aerosol Science 40 (2), 134-151.

Hair, J., Caldwell, L., Krueger, D., She, C., 2001. High-spectral-resolution lidar with iodine - vapor filters: measurement of atmospheric-state and aerosol profiles. Applied Optics 40, 5280-5294.

Hak, C., Pundt, I., Trick, S., Kern, C., Platt, U., Dommen, J., Ordóñez, C., Prévôt, A.S.H., Junkermann, W., Astorga-Lloréns, C., Larsen, B.R., Mellqvist, J., Strandberg, A., Yu, Y., Galle, B., Kleffmann, J., Lörzer, J.C., Braathen, G.O., Volkamer, R., 2005 Intercomparison of four different in situ techniques for ambient formaldehyde measurements in urban air. Atmospheric Chemistry and Physics 5, 28812900

Hallar, A.G., Strawa, A.W., Schmid, B., Andrews, E., Ogren, J., Sheridan, P., Ferrare, P., Covert, D., Elleman, R., Jonsson, K., Luu, A., 2006. Atmospheric radiation measurements aerosol intensive operating period: comparison of aerosol scattering during coordinated flights. Journal of Geophysical Research 111, D05S07.

Hallquist, M., Wenger, J.C., Baltensperger, U., Rudich, Y., Simpson, D., Claeys, M., Dommen, J., Donahue, N.M., George, C., Goldstein, A.H., Hamilton, J.F., Herrmann, H., Hoffmann, T., Iinuma, Y., Jang, M., Jenkin, M., Jimenez, J.L., Kiendler-Scharr, A., Maenhaut, W., McFiggans, G., Mentel, T., Monod, A., Prevot, A.S.H., Seinfeld, J.H., Surratt, J.D., Szmigielski, R., Wildt, J., 2009. The formation, properties and impact of secondary organic aerosol: current and emerging issues. Atmospheric Chemistry and Physics 9, 5155-5235.

Hammer, S., Wagenbach, D., Preunkert, S., Pio, C., Schlosser, C., Meinhardt, F., 2007. Lead-210 observations within CARBOSOL: a diagnostic tool for assessing the spatiotemporal variability of related chemical aerosol species? Journal of Geophysical Research 112, D23S03.

Hanford, K.L., Mitchem, L., Reid, J.P., Clegg, S.L., Topping, D.O., McFiggans, G.B., 2008 Comparative thermodynamic studies of aqueous glutaric acid, ammonium sulfate and sodium chloride aerosol at high humidity. Journal of Physical Chemistry 112, 9413-9422.

Hanke, M., Uecker, J., Reiner, T., Arnold, F., 2002. Atmospheric peroxy radicals: ROXMAS, a new mass-spectrometric methodology for speciated measurements of $\mathrm{HO}_{2}$ and $\sum \mathrm{RO}_{2}$ and first results. International Journal of Mass Spectrometry 213, 91-99.

Hannigan, J.W., Coffey, M.T., Goldman, A. Semi-autonomous NDACC FTS observation system for remote sensing of stratospheric and tropospheric gases. Journal of Atmospheric and Oceanic Technology, in press. doi:10.1175/2009JTECHA1230.1.

Hartogh, P., Espy, P.., 2006. MIPAS (Michelson Interferometer for Passive Atmosphere Sounding): potential of the experiment, data processing and validation of results. Special Issue in Atmospheric Chemistry and Physics.

Hartz, K.E.H., Rosenorn, T., Ferchak, S.R., Raymond, T.M., Bilde, M., Donahue, N.M., Pandis, S.N., 2005. Cloud condensation nuclei activation of monoterpene and sesquiterpene secondary organic aerosol. Journal of Geophysical Research 110, D14208.

Hartz, K.E.H., Weitkamp, E.A., Sage, A.M., Donahue, N.M., Robinson, A.L. 2007. Laboratory measurements of the oxidation kinetics of organic aerosol mixtures using a relative rate constants approach. Journal of Geophysical Research 112, D04204.

Haywood, J.M., Bush, M., Osborne, S.R., Abel, S.J., Claxton, B., Macpherson, B., Harrison, M., Crosier, J., Coe, H., 2008. Prediction of visibility and aerosol within the operational Met Office Unified Model. II: validation of model performance using observational data. Quarterly Journal of the Royal Meteorological Society 134, 1817-1832.

Heald, C.L., Jacob, D.J., Fiore, A.M., Emmons, L.K., Gille, J.C., Deeter, M.N., Warner, J., Edwards, D.P., Crawford, J.H., Hamlin, A.J., Sachse, G.W., Browell, E.V., Avery, M.A., Vay, S.A., Westberg, D.J., Blake, D.R., Singh, H.B., Sandholm, S.T., Talbot, R.W., Fuelberg, H.E., 2003. Asian outflow and trans-Pacific transport of carbon monoxide and ozone pollution: an integrated satellite, aircraft, and model perspective. Journal of Geophysical Research - Atmospheres 108, 4804

Heckel, A., Richter, A., Tarsu, T., Wittrock, F., Hak, C., Pundt, I., Junkermann, W. Burrows, J.P., 2005. MAX-DOAS measurements of formaldehyde in the PoValley. Atmospheric Chemistry and Physics 5, 909-918.

Hegg, D.A., Covert, D.S., Jonsson, H., Covert, P.A., 2005. Determination of the transmission efficiency of an aircraft aerosol inlet. Aerosol Science and Technology 39, 966-971.

Heintzenberg, J., Hermann, M., Theiss, D., 2003. Out of Africa: high aerosol concentrations in the upper troposphere over Africa. Atmospheric Chemistry and Physics 3, 1191-1198.

Heintzenberg J. Wiedensohler, A, Tuch, TM Covert, D. S, Sheridan, P Ogren, J.A Gras, J., Nessler, R., Kleefeld, C., Kalivitis, N., Aaltonen, V., Wilhelm, R.-T., Havlicek, M., 2006. Intercomparisons and aerosol calibrations of 12 commercial integrating nephelometers of 3 manufacturers. Journal of Atmospheric and Oceanic Technology 23, 902-914.

Heland, J., Kleffmann, J., Kurtenbach, R., Wiesen, P., 2001. A new instrument to measure gaseous nitrous acid (HONO) in the atmosphere. Environmental Science and Technology 35, 3207-3212.

Helmig, D., 1997. Ozone removal techniques in the sampling of atmospheric volatile organic trace gases. Atmospheric Environment 31, 3635-3651.

Helsper, C., Horn, H.G., Schneider, F., Wehner, B., Wiedensohler, A., 2009. Intercomparison of Five mobility size spectrometers for measuring atmospheric submicrometer aerosol particles. Gefahrstoffe Reinhaltung der Luft 68(11-12), 475-481. 
Henne, S., Dommen, J., Neininger, B., Reimann, S., Staehelin, J., Prevot, A.S.H., 2005. Influence of mountain venting in the Alps on the ozone chemistry of the lower free troposphere and the European pollution export. Journal of Geophysical Research - Atmospheres 110, D22307.

Henne, S., Folini, D., Brunner, D., Buchmann, B., 2008. Report on Supersite Representativeness and Representativeness Assessment Method. Report for EC FP6 Project GEOmon (Project no. 036677). Empa, Dübendorf, Switzerland. GEOmon D2.1.2.

Henne, S., Klausen, J., Junkermann, W., Kariuki, J.M., Aseyo, J.O., Buchmann, B., 2008. Representativeness and climatology of carbon monoxide and ozone at the global GAW station Mt. Kenya in equatorial Africa. Atmospheric Chemistry and Physics 8, 3119-3139.

Hering, S.V., Stolzenburg, M.R., Quant, F.R., Oberreit, D.R., Keady, P.B., 2005. A laminar-flow, water-based condensation particle counter (WCPC). Aerosol Science and Technology 39, 659-672.

Hering, S.V., Stolzenburg, M.R., 2005. A method for particle size amplification by water condensation in a laminar, thermally diffusive flow. Aerosol Science and Technology 39, 428-436.

Herman, J.R., Barthia, P.K., Torres, O., Hsu, C., Seftor, C., Celarier, E., 1997. Global distribution of UV-absorbing aerosols from Nimbus 7/TOMS data. Journal of Geophysical Research 102, 16911-16922.

Herman, M., Deuzé, J.L., Marchand, A., Roger, B., Lallart, P., 2005. Aerosol remote sensing from POLDER/ADEOS over the ocean. Improved retrieval using nonspherical particle model. Journal of Geophysical Research 110, D10S02.

Hermann, M., Adler, S., Caldow, R., Stratmann, F., Wiedensohler, A., 2005. Pressuredependent efficiency of a condensation particle counter operated with FC-43 as working fluid. Journal of Aerosol Science 36, 1322-1337.

Herndon, S.C., Zahniser, M.S., Nelson, D.D., Shorter, J., McManus, J.B., Jiménez, R., Warneke, C., De Gouw, J.A., 2007. Airborne measurements of $\mathrm{HCHO}$ and $\mathrm{HCOOH}$ during the New England Air Quality Study 2004 using a pulsed quantum cascade laser spectrometer. Journal of Geophysical Research 112, D10S03.

Hertel, O., Ellermann, T., Palmgren, F., Berkowicz, R., Lofstrom, P., Frohn, L.M., Geels, C., Skjoth, C.A., Brandt, J., Christensen, J., Kemp, K., Ketzel, M., 2007. Integrated air-quality monitoring - combined use of measurements and models in monitoring programmes. Environmental Chemistry 4, 65-74.

Hertel, O., Mukai, H., Sawa, Y., Matsueda, H., Yonemura, S., Wang, T., Poon, S., Wong, A., Lee, G., Jung, J., Kim, K., Lee, M., 2007. Direct assessment of measurements and models in Asia. Journal of Environmental Monitoring 9,1183.

Hewison, T., 2006. Profiling temperature and humidity by ground-based microwave radiometers. Ph.D. thesis. Department of Meteorology, University of Reading GB.

Hinz, K.P., Spengler, B., 2007. Instrumentation, data evaluation and quantification in on-line aerosol mass spectrometry. Journal of Mass Spectrometry 42, 843-860.

Holben, B.N., Eck, T.F., Slutsker, I., Tanré, D., Buis, J.P., Setzer, A., Vermote, E., Reagan, J.A., Kaufman, Y.J., Nakajima, T., Lavenu, F., Jankowiak, I., Smirnov, A. 1998. AERONET - a federated instrument network and data archive for aerosol characterization. Remote Sensing of Environment 66, 1-16.

Hofzumahaus, A., Rohrer, F., Lu, K., Bohn, B., Brauers, T., Chang, C.-C., Fuchs, H., Holland, F., Kita, K., Kondo, Y., Li, X., Lou, S., Shao, M., Zeng, L., Wahner, A. Zhang, Y., 2009. Amplified Trace Gas Removal in the Troposphere. Science 324 (5935), 1702. doi:10.1126/science.1164566.

Holben, B.N., Eck, T., Slutsker, I., Smirnov, A., Sinyuk, A., Schafer, J., Giles, D. Dubovik, O., 2006. AERONET Version 2.0 Quality Assurance Criteria. SPIE, Goa, India.

Hollingsworth, A., Engelen, R.J., Textor, C., Benedetti, A., Boucher, O., Chevallier, F.,

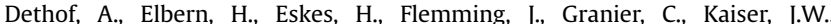
Morcrette, J.-J., Rayner, P., Peuch, V.-H., Rouil, L., Schultz, M.G., Simmons, A.J., GEMS Consortium, 2008. Toward a monitoring and forecasting system for atmospheric composition: the GEMS project. Bulletin of the American Meteorological Society $89,1147$.

Holt, T., Atkinson, R., Arey, J., 2005. Effect of water vapor concentration on the conversion of a series of 1,4-hydroxycarbonyls to dihydrofurans. Journal of Photochemistry and Photobiology A: Chemistry 176, 231-237.

Holzinger, R., Lee, A., McKay, M., Goldstein, A.H., 2006. Seasonal variability of monoterpene emission factors for a Ponderosa pine plantation in California. Atmospheric Chemistry and Physics 6, 1267-1274.

Hönninger, G., von Friedeburg, C., Platt, U., 2004. Multi axis differential optical absorption spectroscopy (MAX-DOAS). Atmospheric Chemistry and Physics 4, 231-254.

Hopkins, R.J., 2004. Control and characterisation of a single aerosol droplet in a single-beam gradient-force optical trap. Physical Chemistry and Chemical Physics 6, 4924-4927.

Hopkins, J.R., Lewis, A.C., Read, K.A., 2003. A two-column method for long-term monitoring of non-methane hydrocarbons (NMHCs) and oxygenated volatile organic compounds (o-VOCs). Journal of Environmental Monitoring 5, 8-13.

Hopkins, J.R., Still, T., Al-Haider, S., Fischer, I.R., Lewis, A.C., Seakins, P.W., 2003. A simplified apparatus for ambient formaldehyde detection via GC-pHID. Atmospheric Environment 37, 2557-2565.

Howle, C.R., Homer, C.J., Hopkins, R.J., Reid, J.P., 2007. Probing the evaporation of ternary ethanol-methanol-water droplets by cavity enhanced Raman scattering. Physical Chemistry and Chemical Physics 9, 5344-5352.

Hu, Y., Vaughan, M., McClain, C., Behrenfeld, M., Maring, H., Anderson, D., SunMack, S., Flittner, D., Huang, J., Wielicki, B., Minnis, P., Weimer, C., Trepte, C. Kuehn, R., 2007. Global statistics of liquid water content and effective number concentration of water clouds over ocean derived from combined CALIPSO and MODIS measurements. Atmospheric Chemistry and Physics 7, 3353-3359.
Huang, L., Brook, J.R., Zhang, W., Li, S.M., Graham, L., Ernst, D., Chivulescu, A., Lu, G. 2006. Stable isotope measurements of carbon fractions (OC/EC) in airborne particulate: a new dimension for source characterization and apportionment. Atmospheric Environment 40, 2690-2705.

Huey, L.G., Tanner, D.J., 2006. Measurement of trace atmospheric gases by chemical ionization mass spectrometry. In: Heard, D. (Ed.), Free Radicals in the Troposphere, ACCENT Report 6.2006, Sep. 2006. ACCENT Secretariat, Urbino, Italy, pp. 76-78.

Huffman, J.A., Jayne, J.T., Drewnick, F., Aiken, A.C., Onasch, T., Worsnop, D.R. Jimenez, J.L., 2005. Design, modeling, optimization, and experimental tests of a particle beam width probe for the Aerodyne aerosol mass spectrometer Aerosol Science and Technology 39, 1143-1163.

Huffman, J.A., Ziemann, P.J., Jayne, J.T., Worsnop, D.R., Jimenez, J.L., 2008. Development and characterization of a fast-stepping/scanning thermodenuder for chemically-resolved aerosol volatility measurements. Aerosol Science and Technology 42, 395-407.

Husar, R.B., Prospero, J.M., Stowe, L.L., 1997. Characterization of tropospheric aerosols over the oceans with the NOAA advanced very high resolution radiomete optical thickness operational product. Journal of Geophysical Research 102, 16889-16909.

IGACO Theme Team, 2004. The Changing Atmosphere, an Integrated Global Atmospheric Chemistry Observation Theme for the IGOS Partnership. ESA SP1282, GAW Report No. 159 (WMO TD No. 1235). European Space Agency, ESA Noordwijk, The Netherlands.

Iinuma, Y., Muller, C., Berndt, T., Boge, O., Claeys, M., Herrmann, H., 2007. Evidence for the existence of organosulfates from beta-pinene ozonolysis in ambient secondary organic aerosol. Environmental Science and Technology 41, 6678-6683.

Illingworth, A.J., Hogan, R.J., Connor, E.J., Bouniol, D., Brooks, M.E., Delanoë, J Donovan, D.P., Eastment, J.D., Gaussiat, N., Goddard, J.W.F., Haeffelin, M. Baltink, H.K., Krasnov, O.A., Pelon, J., Piriou, J.M., Protat, A., Russchenberg, H.W.J., Seifert, A., Tompkins, A.M., van Zadelhoff, G.J., Vinit, F., Willén, U., Wilson, D.R., Wrench, C.L., 2007. CloudNet: continuous evaluations of cloud profiles in seven operational models using ground-based observations. Bulletin of the American Meteorological Society 88, 883-898.

Inomata, S., Tanimoto, H., Kameyama, S., Tsunogai, U., Irie, H., Kanaya, Y., Wang, Z. 2008. Technical note: determination of formaldehyde mixing ratios in air with PTR-MS: laboratory experiments and field measurements. Atmospheric Chemistry and Physics 8, 273-284.

Irie, H., Kanaya, Y., Akimoto, H., Iwabuchi, H., Shimizu, A., Aoki, K., 2008. First retrieval of tropospheric aerosol profiles using MAX-DOAS and comparison with lidar and sky radiometer measurements. Atmospheric Chemistry and Physics 8, 341-350.

Isaksen, I.S.A., Benestad, R., Berntsen, T.K., Bousquet, P., Collins, B., Cox, T., Dalsoren, S.B., Eyring, V., Fowler, D., Fuzzi, S., Gauss, M., Granier, C., Joeckel, P., Klimont, Z., Laj, P. Lohmann, U., Maione, M., Monks, P., Myhre, G., Prevot, A.S.H., Raes, F. Richter, A., Rognerud, B., Schulz, M., Shindell, D., Stevenson, D., Storelvmo, T. Wang, W.-C., van Weele, M., Wild, M., Wuebbles, D. Atmospheric composition change - climate chemistry interaction, in this issue.

ISO, 2003. Geographic Information - Metadata (ISO 19115:2003). International Organization for Standardization, Geneva, Switzerland.

Jacobi, H., Kaleschke, A., Richter, A., Rozanov, A., Burrows, J.P., 2006. Observation of a fast ozone loss in the marginal ice zone of the Arctic Ocean. Journal of Geophysical Research 111, D15309.

Jacobson, M.C., Hansson, H.-C., Noone, K.J., Charlson, R.J., 2000. Organic atmospheric aerosols: review and state of the science. Reviews of Geophysics 38, 267-294.

Jaegle, L., Steinberger, L., Martin, R.V., Chance, K., 2005. Global partitioning of NO sources using satellite observations: relative roles of fossil fuel combustion, biomass burning and soil emissions. Faraday Discussions 130, 407-423.

Jaffe, D., Price, H., Parrish, D., Goldstein, A., Harris, J., 2003. Increasing background ozone during spring on the west coast of North America. Geophysical Research Letters 30.

Jaffe, D., Ray, J., 2007. Increase in surface ozone at rural sites in the western US Atmospheric Environment 41, 5452-5463.

Janis, M.J., Hubbard, K.G., Redmond, K.T., 2004. Station density strategy for monitoring long-term climatic change in the contiguous United States. Journal of Climate 17, 151-162.

Jankowiak, I., Tanré, D., 1992. Satellite climatology of Saharan dust outbreaks: method and preliminary results. Journal of Climate 5, 646-656.

Jaoui, M., Kleindienst, T.E., Lewandowski, M., Edney, E.O., 2004. Identification and quantification of aerosol polar oxygenated compounds bearing carboxylic or hydroxyl groups. 1. Method development. Analytical Chemistry 76, 4765-4778.

Jayne, J.T., Leard, D.C., Zhang, X.F., Davidovits, P., Smith, K.A., Kolb, C.E. Worsnop, D.R., 2000. Development of an aerosol mass spectrometer for size and composition analysis of submicron particles. Aerosol Science and Technology 33, 49-70.

Jiang, J.H., Livesey, N.J., Su, H., Neary, L., McConnell, J.C., Richards, N.A.D., 2007 Connecting surface emissions, convective uplifting, and long-range transport of carbon monoxide in the upper troposphere: new observations from the Aura Microwave Limb Sounder. Geophysical Research Letters 34, L18812.

Jimenez, J.L., Jayne, J.T., Shi, Q., Kolb, C.E., Worsnop, D.R., Yourshaw, I., Seinfeld, J.H. Flagan, R.C., Zhang, X., Smith, K.A., Morris, J.W., Davidovits, P., 2003. Ambient aerosol sampling using the Aerodyne aerosol mass spectrometer. Journal of Geophysical Research - Atmospheres 108, 8425.

Johanson, T., Caldow, R., Pöcher, A., Mirme, A., Kittelson, D., 2004. A new electrical mobility particle sizer spectrometer for engine exhaust particle measurements. Society of Automotive Engineers Technical Paper Series 2004-01-1341. 
Jones, H., Abbatt, J., Bowles, J., Cotton, R., DeMott, P.J., Kanji, Z., Möhler, O., Petters, M., Saunders, C., Sierau, B., Stetzer, O., 2008. Initial results from the Manchester ice nucleation counter taken during ICIS2007. In: The Proceedings of the International Conference on Clouds and Precipitation, Cancun, Mexico, July 711.

Jonhson, G.R., Ristovski, Z., Morawska, L., 2004. Method for measuring the hygroscopic behaviour of lower volatility fractions in an internally mixed aerosol. Journal of Aerosol Science 35, 443-455.

Jourdain, L., Worden, H.M., Worden, J.R., Bowman, K., Li, Q., Eldering, A., Kulawik, S.S., Osterman, G., Boersma, K.F., Fisher, B., Rinsland, C.P., Beer, R. Gunson, M., 2007. Tropospheric vertical distribution of tropical Atlantic ozone observed by TES during the northern African biomass burning season. Geophysical Research Letters 34.

Joutsensaari, J., Toivonen, T., Vattovaara, P., Vesterinen, M., Vepsalainen, J., Laaksonen, A., 2004. Time-resolved growth behavior of acid aerosols in ethano vapor with a tandem-DMA technique. Journal of Aerosol Science 35, 851-867.

Joutsensaari, J., Loivamäki, M., Vuorinen, T., Miettinen, P., Nerg, A.-M., Holopainen, J.K., Laaksonen, A., 2005. Nanoparticle formation by ozonolysis of inducible plant volatiles. Atmospheric Chemistry and Physics 5.

Junkermann, W., Burger, J.M., 2006. A new portable instrument for continuous measurement of formaldehyde in ambient air. Journal of Atmospheric and Oceanic Technology 23, 38-45.

Kahn, R., Banerjee, P., McDonald, D., 2001. The sensitivity of multiangle imaging to natural mixtures of aerosols over ocean. Journal of Geophysical Research 106 18219-18238.

Kahn, R.A., Garay, M.J., Nelson, D.L., Yau, K.K., Bull, M.A., Gaitley, B.J., Martonchik, J.V. Levy, R.C., 2007. Satellite-derived aerosol optical depth over dark water from MISR and MODIS: comparison with AERONET and implication for climatological studies. Journal of Geophysical Research 112, D18205.

Kahnert, M., 2008. Variational data analysis of aerosol species in a regional CTM: background error covariance constraint and aerosol optical observation operators. Tellus B 60, 753-770.

Kaiser, J., Hastings, M.G., Houlton, B.Z., Röckmann, T., Sigman, D.M., 2007. Triple oxygen isotope analysis of nitrate using the denitrifier method and therma decomposition of $\mathrm{N}_{2} \mathrm{O}$. Analytical Chemistry 79, 599-607.

Kalabokas, P.D., Volz-Thomas, A., Brioude, J., Thouret, V., Cammas, J.-P., Repapis, C.C., 2007. Vertical ozone measurements in the troposphere over the Eastern Mediterranean and comparison with Central Europe. Atmospheric Chemistry and Physics 7, 3783-3790.

Kalberer, M., Paulsen, D., Sax, M., Steinbacher, M., Dommen, J., Prevot, A.S.H., Fisseha, R., Weingartner, E., Frankevich, V., Zenobi, R., Baltensperger, U., 2004 Identification of polymers as major components of atmospheric organic aerosols. Science 303, 1659-1662.

Kaleschke, L., Richter, A., Burrows, J., Afe, O., Heygster, G., Notholt, J., Rankin, A.M. Roscoe, H.K., Hollwedel, J., Wagner, T., Jacobi, H.W., 2004. Frost flowers on sea ice as a source of sea salt and their influence on tropospheric halogen chemistry. Geophysical Research Letters 31, L16114.

Kämpfer, N., Mätzler, C., Brocard, E., Feist, D., Haefele, A., Hocke, K., Martin, L. Morland, J., Müller, S., Schneebeli, M., 2006. Microwave remote sensing of the atmosphere - a University profile. IEEE Geoscience and Remote Sensing Society Newsletter 140, 13-17.

Kanakidou, M., Seinfeld, J.H., Pandis, S.N., Barnes, I., Dentener, F.J., Facchini, M.C., Van Dingenen, R., Ervens, B., Nenes, A., Nielsen, C.J., Swietlicki, E., Putaud, J.P., Balkanski, Y., Fuzzi, S., Horth, J., Moortgat, G.K., Winterhalter, R., Myhre, C.E.L. Tsigaridis, K., Vignati, E., Stephanou, E.G., Wilson, J., 2005. Organic aerosol and global climate modelling: a review. Atmospheric Chemistry and Physics 5, 1053-1123.

Kang, E., Root, M.J., Toohey, D.W., Brune, W.H., 2007. Introducing the concept of Potential Aerosol Mass (PAM). Atmospheric Chemistry and Physics 7, 5727-5744.

Kanji, Z.A., Abbatt, J.P.D., 2006. Laboratory studies of ice formation via deposition mode nucleation onto mineral dust and n-hexane soot samples. Journal of Geophysical Research 111, D16204.

Kannosto, J., Virtanen, A., Lemmetty, M., Mäkelä, J.M., Keskinen, J., Junninen, H. Hussein, T., Aalto, P., Kulmala, M., 2008. Mode resolved density of atmospheric aerosol particles. Atmospheric Chemistry and Physics 8, 5327-5337.

Kar, J., Bremer, H., Drummond, J.R., Rochon, Y.J., Jones, D.B.A., Nichitiu, F., Zou, J., Liu, J., Gille, J.C., Edwards, D.P., Deeter, M.N., Francis, G., Ziskin, D., Warner, J. 2004. Evidence of vertical transport of carbon monoxide from Measurements of Pollution in the Troposphere (MOPITT). Geophysical Research Letters 31.

Kar, J., Drummond, J.R., Jones, D.B.A., Liu, J., Nichitiu, F., Zou, J., Gille, J.C. Edwards, D.P., Deeter, M.N., 2006. Carbon monoxide (CO) maximum over the Zagros mountains in the Middle East: signature of mountain venting? Geophysical Research Letters 33.

Karl, T., Guenther, A., Jordan, A., Fall, R., Lindinger, W., 2000. Eddy covariance measurement of biogenic oxygenated VOC emissions from hay harvesting. Atmospheric Environment 35.

Karl, T., Crutzen, P.J., Mandl, M., Staudinger, M., Guenther, A., Jordan, A., Fall, R., Lindinger, W., 2001. Variability-lifetime relationship of VOCs observed at the Sonnblick Observatory 1999-estimation of HO-densities. Atmospheric Environment 35, 5287-5300.

Karl, T., Fall, R., Crutzen, P.J., Jordan, A., Lindinger, W., 2001. High concentrations of reactive biogenic VOCs at a high altitude site in late autumn. Geophysical Research Letters 28, 507-510.

Karl, T.G., Spirig, C., Rinne, J., Stroud, C., Prevost, P., Greenberg, J., Fall, R., Guenther, A., 2002. Virtual disjunct eddy covariance measurements of organic compound fluxes from a subalpine forest using proton transfer reaction mass spectrometry. Atmospheric Chemistry and Physics 2, 279-291.

Karl, T., Potosnak, M., Guenther, A., Clark, D., Walker, J., Herrik, J.D., Geron, C., 2004. Exchange processes of volatile organic compounds above a tropical rain forest: implications for modeling tropospheric chemistry above dense vegetation. Journal of Geophysical Research 109, D18306.

Karunanandan, R., Hölscher, D., Dillon, T.J., Horowitz, A., Crowley, J.N., 2007. Reaction of $\mathrm{HO}$ with glycolaldehyde, $\mathrm{HOCH}_{2} \mathrm{CHO}$ : rate coefficients (240-362 K) and mechanism. Journal of Physical Chemistry 111, 897-908.

Katrib, Y., Biskos, G., Buseck, P.R., Davidovits, P., Jayne, J.T., Mochida, M., Wise, M.E., Worsnop, D.R., Martin, S.T., 2005. Ozonolysis of mixed oleic-acid/stearic-acid particles: reaction kinetics and chemical morphology. Journal of Physical Chemistry - Atmospheres 109, 10910-10919.

Kaufman, Y., Tanré, D., Remer, L., Vermote, D., Chu, A., Holben, B., 1997. Operational remote sensing of tropospheric aerosol over land from EOS moderate resolution imaging spectroradiometer. Journal of Geophysical Research 102, 1705117067.

Kaufman, Y.J., Koren, I., 2006. Smoke and pollution aerosol effect on cloud cover. Science 313, 655-658.

Kaufmann, Y.F., Holben, B.N., Tanré, D., Slutsker, I., Smirnov, A., Eck, T.F., 2000. Will aerosol measurements from Terra and Aqua polar orbiting satellites represent the daily aerosol abundance and properties? Geophysical Research Letters 27, 3861-3864.

Kawamura, K., Watanabe, T., 2004. Determination of stable carbon isotopic compositions of low molecular weight dicarboxylic acids and ketocarboxylic acids in atmospheric aerosol and snow samples. Analytical Chemistry 76, 5762-5768.

Khlystov, A., Wyers, G.P., Slanina, J., 1995. The steam-jet aerosol collector. Atmospheric Environment 29, 2229-2234.

Kim, C.S., Okuyama, K., de la Mora, F., 2003. Performance evaluation of an improved particle size magnifier (PSM) for single nanoparticle detection. Aerosol Science and Technology 37, 791-803.

Kim, S.W., Heckel, A., McKeen, S.A., Frost, G.J., Hsie, E.Y., Trainer, M.K., Richter, A., Burrows, J.P., Peckham, S.E., Grell, G.A., 2006. Satellite-observed US power plant $\mathrm{NO}_{\mathrm{x}}$ emission reductions and their impact on air quality. Geophysical Research Letters 33.

Kim, S.W., Yoon, S.C., Dutton, E.G., Kim, J., Wehrli, C., Holben, B.N., 2008. Global surface-based sun photometer network for long-term observations of column aerosol optical properties: intercomparison of aerosol optical depth. Aerosol Science and Technology 42,1-9.

King, M., Kaufman, Y., Menzel, P., Tanré, D., 1992. Remote sensing of cloud, aerosol and water vapor properties from the moderate resolution imaging spectrometer (MODIS). IEEE Transactions on Geoscience and Remote Sensing 30, 2-27.

King, S.M., Rosenoern, T., Shilling, J.E., Chen, Q., Martin, S.T., 2007. Cloud condensation nucleus activity of secondary organic aerosol particles mixed with sulfate. Geophysical Research Letters 34, L06708.

Kinne, S., Schulz, M., Textor, C., Guibert, S., Balkanski, Y., Bauer, S.E., Berntsen, T., Berglen, T.F., Boucher, O., Chin, M., Collins, W., Dentener, F., Diehl, T., Easter, R., Feichter, J., Fillmore, D., Ghan, S., Ginoux, P., Gong, S., Grini, A., Hendricks, J., Herzog, M., Horowitz, L., Isaksen, I., Iversen, T., Kirkevåg, A., Kloster, S., Koch, D., Kristjansson, J.E., Krol, M., Lauer, A., Lamarque, J.F., Lesins, G., Liu, X., Lohmann, U., Montanaro, V., Myhre, G., Penner, J., Pitari, G., Reddy, S., Seland, O., Stier, P., Takemura, T., Tie, X., 2006. An AeroCom initial assessment optical properties in aerosol component modules of global models. Atmospheric Chemistry and Physics 6, 1815-1834

Klausen, J., Zellweger, C., Buchmann, B., Hofer, P., 2003. Uncertainty and bias of surface ozone measurements at selected global atmosphere watch sites. Journal of Geophysical Research - Atmospheres 108, 4622. doi:10.1029/2003JD003710.

Kleffmann, J., 2007. Daytime sources of nitrous acid (HONO) in the atmospheric boundary layer. ChemPhysChem 8, 1137-1144.

Kleffmann, J., Lörzer, J.C., Wiesen, P., Kern, C., Trick, S., Volkamer, R., Rodenas, M., Wirtz, K., 2006. Intercomparison of the DOAS and LOPAP techniques for the detection of nitrous acid (HONO). Atmospheric Environment 40, 3640-3652.

Kleffmann, J., Gavriloaiei, T., Elshorbany, Y., Ródenas, M., Wiesen, P., 2007. Detection of nitric acid $\left(\mathrm{HNO}_{3}\right)$ in the atmosphere using the LOPAP technique. Journal of Atmospheric Chemistry 58, 131-149.

Klein, H., Bundke, U., Nillius, B., Schütz, L., Wetter, T., Bingemer, H., 2008. The variability of ice nucleating aerosols over central Europe. In: International Conference on Clouds and Precipitation, Cancun, Mexico.

Knopf, D.A., Koop, T., 2006. Heterogeneous nucleation of ice on surrogates of mineral dust. Journal of Geophysical Research 111, D12201.

Kobayashi, H., Shimota, A., Kondo, K., Okumura, E., Kameda, Y., Shimoda, H., Ogawa, T., 1999. Development and evaluation of the interferometric monitor for greenhouse gases: a high-throughput Fourier-transform infrared radiometer for nadir Earth observation. Applied Optics 38, 6801-6807.

Kolgotin, A., Müller, D., 2008. Two-dimensional regularization for the retrieval of profiles of microphysical particle properties from multiwavelength Raman lidar observations, theory. Applied Optics 47, 4472-4490.

Komatsu, D.D., Ishimura, T., Nakagawa, F., Tsunogai, U., 2008. Determination of the ${ }^{15} \mathrm{~N} /{ }^{14} \mathrm{~N},{ }^{17} \mathrm{O} /{ }^{16} \mathrm{O}$, and ${ }^{18} \mathrm{O} /{ }^{16} \mathrm{O}$ ratios of nitrous oxide by using continuous-flow isotope-ratio mass spectrometry. Rapid Communications in Mass Spectrometry 22, 1587-1596.

Komenda, M., Schaub, A., Koppmann, R., 2003. Description and characterization of an on-line system for long-term measurements of isoprene, methyl vinyl ketone, and methacrolein in ambient air. Journal of Chromatography A 995, 185-201. 
Konovalov, I.B., Beekmann, M., Vautard, R., Burrows, J.P., Richter, A., Nuss, H., Elansky, N., 2005. Comparison and evaluation of modelled and GOME measurement derived tropospheric $\mathrm{NO}_{2}$ columns over Western and Eastern Europe. Atmospheric Chemistry and Physics 5, 169-190.

Konovalov, I.B., Beekmann, M., Richter, A., Burrows, J.P., 2006. Inverse modelling of the spatial distribution of $\mathrm{NO}_{\mathrm{x}}$ emissions on a continental scale using satellite data. Atmospheric Chemistry and Physics 6, 1747-1770.

Konovalov, I.B., Beekmann, M., Burrows, J.P., Richter, A., 2008. Satellite measurement based estimates of decadal changes in European nitrogen oxides emissions. Atmospheric Chemistry and Physics 8, 2623-2641.

Koop, T., Luo, B., Tsias, A., Peter, T., 2000. Water activity as the determinant for homogeneous ice nucleation in aqueous solutions. Nature 406, 611-614.

Koponen, I.K., Riipinen, I., Hienola, A., Kulmala, M., Bilde, M., 2007. Thermodynamic properties of malonic, succinic, and glutaric acids: evaporation rates and saturation vapor pressures. Environmental Science and Technology 41, 3926-3933.

Koppmann, R., Johnen, F.J., Khedim, A., Rudolph, J., Wedel, A., Wiards, B., 1995. The influence of ozone on light nonmethane hydrocarbons during cryogenic preconcentration. Journal of Geophysical Research 100, 11383-11391.

Koren, I., Remer, L.A., Kaufman, Y.J., Rudich, Y., Martins, J.V., 2007. On the twilight zone between clouds and aerosols. Geophysical Research Letters 34, L08805.

Koren, I., Martins, J.V., Remer, L.A., Afargan, H., 2008. Smoke invigoration versus inhibition of clouds over the Amazon. Science 321, 946-949.

Kormann, R., Fischer, H., Gurk, C., Helleis, F., Klüpfel, T., Kowalski, K., Königstedt, R., Parchatka, U., Wagner, V., 2002. Application of a multi-laser tunable diode laser absorption spectrometer for atmospheric trace gas measurements at sub-ppbv levels. Spectrochimica Acta Part A - Molecular and Biomolecular Spectroscopy 58, 2489-2498.

Kornexl, B.E., Gehre, M., Höfling, R., Werner, R.A., 1999. On-line $\delta^{18}$ O measurement of organic and inorganic substances. Rapid Communications in Mass Spectrometry $13,1685-1693$.

Kovacs, T.A., Brune, W.H., 2001. Total OH loss rate measurement. Journal of Atmospheric Chemistry 39, 105.

Krämer, M., Afchine, A., 2004. Sampling characteristics of inlets operated at low U/ Uo ratios: new insights from computational fluid dynamics (CFX) modelling. Journal of Aerosol Science 35, 683-694.

Krasnoperov, L.N., Chesnokov, E.N., Stark, H., Ravishankara, A.R., 2005. Elementary reactions of formyl (HCO) radical studied by laser photolysis-transient absorption spectroscopy. Proceedings of the Combustion Institute 30.

Krueger, A.J., Walter, L.S., Bhartia, P.K., Schnetzler, C.C., Krotkov, N.A., Sprod, I., Bluth, G.J.S., 1995. Volcanic sulfur-dioxide measurements from the total ozone mapping spectrometer instruments. Journal of Geophysical Research - Atmospheres 100, 14057-14076.

Krueger, A.J., Jaross, G., 1999. TOMS ADEOS instrument characterization. IEEE Transactions on Geoscience and Remote Sensing 37, 1543-1549.

Kulmala, M., Mordas, G., Petäjä, T., Grönholm, T., Aalto, P.P., Vehkamäki, H., Hienola, A.I., Herrmann, E., Sipilä, M., Riipinen, I., Manninen, H.E., Hämeri, K., Stratmann, F., Bilde, M., Winkler, P.M., Birmili, W., Wagner, P.E., 2007. The condensation particle counter battery $(\mathrm{CPCB})$ : a new tool to investigate the activation properties of nanoparticles. Journal of Aerosol Science 38, 289-304.

Kulmala, M., Riipinen, I., Sipilä, M., Manninen, H.E., Petäjä, T., Junninen, H., Dal Maso, M., Mordas, G., Mirme, A., Vana, M., Hirsikko, A., Laakso, L., Harrison, R.M., Hanson, I., Leung, C., Lehtinen, K.E.J., Kerminen, V.-M., 2007. Toward direct measurement of atmospheric nucleation. Science 318, 89-92.

Lahoz, W.A., Geer, A.J., Bekki, S., Bormann, N., Ceccherini, S., Elbern, H., Errera, Q., Eskes, H.J., Fonteyn, D., Jackson, D.R., Khattatov, B., Marchand, M., Massart, S., Peuch, V.-H., Rharmili, S., Ridolfi, M., Segers, A., Talagrand, O., Thornton, H.E., Vik, A.F., von Clarmann, T., 2007. The Assimilation of Envisat data (ASSET) project. Atmospheric Chemistry and Physics 7, 1773-1796.

Laj, P., Flossmann, A.I., Wobrock, W., Fuzzi, S., Orsi, G., Ricci, L., Mertes, S. Schwarzenböck, A., Heintzenberg, J., Ten Brink, H., 2001. Behaviour of $\mathrm{H}_{2} \mathrm{O}_{2}$, $\mathrm{NH}_{3}$, and black carbon in mixed-phase clouds during CIME. Atmospheric Research SI 58, 315-336.

Lamquin, N., Stubenrauch, C.J., Pelon, J., 2008. Upper tropospheric humidity and cirrus geometrical and optical thickness: relationships inferred from 1 year of collocated AIRS and CALIPSO data. Journal of Geophysical Research 113, D00A08.

Lance, S., Medina, J., Smith, J.N., Nenes, A., 2006. Mapping the operation of the DMT continuous flow CCN counter. Aerosol Science and Technology 40, 1-13.

Landgraf, J., Hasekamp, O.P., 2007. Retrieval of ozone: the synergistic use of thermal infrared nadir sounder. Journal of Geophysical Research - Atmospheres 112, D08310.

Lang-Yona, N., Rudich, Y., Segre, E., Dinar, E., Abo Riziq, A., 2009. Complex refractive indices of aerosols retrieved by continuous wave - cavity ring down aerosol spectrometer (CW-CRD-AS). Analytical Chemistry 81, 1762-1769.

Lanz, V.A., Alfarra, M.R., Baltensperger, U., Buchmann, B., Hueglin, C., Prevot, A.S.H., 2007. Source apportionment of submicron organic aerosols at an urban site by factor analytical modelling of aerosol mass spectra. Atmospheric Chemistry and Physics 7, 1503-1522.

Lanz, V.A., Alfarra, M.R., Baltensperger, U., Buchmann, B., Hueglin, C., Szidat, S., Wehrli, M.N., Wacker, L., Weimer, S., Caseiro, A., Puxbaum, H., Prevot, A.S.H., 2008. Source attribution of submicron organic aerosols during wintertime inversions by advanced factor analysis of aerosol mass spectra. Environmental Science and Technology 42, 214-220.

Larssen, S., Sluyter, R., Helmis, C., 1999. Criteria for EUROAIRNET - the EEA air quality monitoring and information network. In: European Environment Agency (Ed.). European Environment Agency, Copenhagen, Denmark, p. 56.
Lawson, R.P., Baker, B.A., Zmarzly, P., O'Connor, D., Mo, Q., Gayet, J.-F., Shcherbakov, V., 2006. Microphysical and optical properties of atmospheric ice crystals at South Pole Station. Journal of Applied Meteorology and Climatology $45,1505-1524$

Le Calve, S., Hitier, D., Le Bras, G., Mellouki, A., 1997. Kinetic studies of OH reactions with a series of ketones. Journal of Physical Chemistry - Atmospheres 102 4579-4584.

Le Calve, S., Le Bras, G., Mellouki, A., 1997. Kinetic studies of OH reactions with a series of methyl esters. Journal of Physical Chemistry - Atmospheres 101, 9137-9141.

Lee, A.K.Y., Ling, T.Y., Chan, C.K., 2008. Understanding hygroscopic growth and phase transformation of aerosols using single particle Raman spectroscopy in an electrodynamic balance. Faraday Discussions 137, 245-263.

Legrand, M., Puxbaum, H., 2007. Summary of the CARBOSOL project: present and retrospective state of organic versus inorganic aerosol over Europe. Journal of Geophysical Research 112, D23S01.

Legreid, G., Loov, J.B., Staehelin, J., Hueglin, C., Hill, M., Buchmann, B., Prevot, A.S.H., Reimann, S., 2007. Oxygenated volatile organic compounds (OVOCs) at an urban background site in Zurich (Europe): seasonal variation and source allocation. Atmospheric Environment 41, 8409-8423.

Leigh, R.J., Corlett, G.K., Friess, U., Monks, P.S., 2006. A concurrent multi-axis differential optical absorption spectroscopy system for the measurement of tropospheric nitrogen dioxide. Applied Optics 45, 7504-7518.

Leigh, R.J., Corlett, G.K., Friess, U., Monks, P.S., 2007. Spatially resolved measurements of nitrogen dioxide in an urban environment using concurrent multi-axis differential optical absorption spectroscopy. Atmospheric Chemistry and Physics 7, 4751-4762.

Levelt, P.F., Van den Oord, G.H.J., Dobber, M.R., Malkki, A., Visser, H., de Vries, J. Stammes, P., Lundell, J.O.V., Saari, H., 2006. The ozone monitoring instrument IEEE Transactions on Geoscience and Remote Sensing 44, 1093-1101.

Lewis, A.C., Carslaw, N., Marriott, P.J., Kinghorn, R.M., Morrison, P.D., Lee, A.L. Bartle, K.D., Pilling, M.J., 2000. A larger pool of ozone-forming carbon compounds in urban atmospheres. Nature 405, 778-781.

Li, Y., Schwab, J.J., Demerjian, K.L., 2006. Measurements of ambient ammonia using a tunable diode laser absorption spectrometer: characteristics of ambient ammonia emissions in an urban area of New York City. Journal of Geophysical Research.

Li, Y.Q., Schwab, J.J., Demerjian, K.L., 2008. Fast time response measurements of gaseous nitrous acid using a tunable diode laser absorption spectrometer: HONO emission source from vehicle exhausts. Geophysical Research Letters 35, L04803.

Lindinger, W., Hansel, A., Jordan, A., 1998. On-line monitoring of volatile organic compounds at pptv levels by means of proton-transfer-reaction mass spectrometry (PTR-MS) - medical applications, food control and environmental research. International Journal of Mass Spectrometry 173, 191-241.

Lindinger, W., Hansel, A., Jordan, A., 1998. Proton-transfer-reaction mass spectrometry (PTR-MS): on-line monitoring of volatile organic compounds at pptv levels. Chemical Society Reviews 27, 347-354.

Ling, T.Y., Chan, C.K., 2008. Partial crystallization and deliquescence of particles containing ammonium sulfate and dicarboxylic acids. Journal of Geophysical Research 113, D14205.

Liu, B.Y.H., Pui, D.Y.H., Whitby, K.T., Kittelson, D.B., Kousaka, Y., McKenzie, R.L., 1978 The aerosol mobility chromatograph: a new detector for sulfuric acid aerosols. Atmospheric Environment 12, 99-104.

Liu, P., Ziemann, P.J., Kittelson, D.B., McMurry, P.H., 1995. Generating particle beams of controlled dimensions and divergence: I. Theory of particle motion in aerodynamic lenses and nozzle expansions. Aerosol Science and Technology 22 293-313.

Liu, P., Ziemann, P.J., Kittelson, D.B., McMurry, P.H., 1995. Generating particle beams of controlled dimensions and divergence: II. Experimental evaluation of particle motion in aerodynamic lenses and nozzle expansions. Aerosol Science and Technology 22, 314-324.

Liu, X., Chance, K., Sioris, C.E., Kurosu, T.P., Spurr, R.J.D., Martin, R.V., Fu, T.M., Logan, J.A., Jacob, D.J., Palmer, P.I., Newchurch, M.J., Megretskaia, I.A., Chatfield, R.B., 2006. First directly retrieved global distribution of tropospheric column ozone from GOME: comparison with the GEOS-CHEM model. Journal of Geophysical Research - Atmospheres 111, D10399.

Liu, X.J., Ju, X.T., Zhang, Y., He, C., Kopsch, J., Fusuo, Z., 2006. Nitrogen deposition in agroecosystems in the Beijing area. Agriculture Ecosystems \& Environment 113 370-377.

Liu, Z., Omar, A., Vaughan, M., Hair, J., Kittaka, C., Hu, Y., Powell, K., Trepte, C. Winker, D., Hostetler, C., Ferrare, R., Pierce, R., 2008. CALIPSO lidar observations of the optical properties of Saharan dust: a case study of long-range transport. Journal of Geophysical Research 113, D07207.

Löhnert, U., Crewell, S., Simmer, C., 2004. An integrated approach towards retrieving physically consistent profiles of temperature, humidity, and cloud liquid water. Journal of Applied Meteorology 43, 1295-1307.

Löhnert, U., Crewell, S., Krasnov, O., O’Connor, E., Russchenberg, H., 2008. Advances in continuously profiling the thermodynamic state of the boundary layer: integration of measurements and methods. Journal of Atmospheric and Oceanic Technology 25, 1251-1266.

Lugauer, M., Baltensperger, U., Furger, M., Gäggeler, H.W., Jost, D.T., Nyeki, S. Schwikowski, M., 2000. Influences of vertical transport and scavenging on aerosol particle surface area and Radon decay product concentrations at the Jungfraujoch (3454 m asl). Journal of Geophysical Research 105, 19869-19879. 
Magee, N., Moyle, A.M., Lamb, D., 2006. Experimental determination of the deposition coefficient of small cirrus-like ice crystals near-50 ${ }^{\circ} \mathrm{C}$. Geophysical Research Letters 33, L17813.

Malinka, A.V., Zege, E.P., 2007. Possibilities of warm cloud microstructure profiling with multiple-field-of-view Raman lidar. Applied Optics 46, 8419-8427.

Mao, J., Ren, X., Brune, W.H., Olson, J.R., Crawford, J.H., Fried, A., Huey, L.G., Cohen, R.C., Heikes, B., Singh, H.B., Blake, D.R., Sachse, G.W., Diskin, G.S., Hall, S.R., Shetter, R.E., 2008. Airborne measurement of $\mathrm{OH}$ reactivity during INTEX-B. Atmospheric Chemistry and Physics 9, 163-173.

Marcolli, C., Gedamke, S., Peter, T., Zobrist, B., 2007. Efficiency of immersion mode ice nucleation on surrogates of mineral dust. Atmospheric Chemistry and Physics 7, 5081-5091.

Marcolli, C., Krieger, U.K., 2006. Phase changes during hygroscopic cycles of mixed organic/inorganic model systems of tropospheric aerosols. Journal of Physical Chemistry - Atmospheres 110, 1881-1893.

Marenco, A., Thouret, V., Nédélec, P., Smit, H., Helten, M., Kley, D., Karcher, F Simon, P., Law, K., Pyle, J., Poschmann, G., Von Wrede, R., Hume, C., Cook, T. 1998. Measurement of ozone and water vapour by airbus in-service aircraft: the MOZAIC airborne program, an overview. Journal of Geophysical Research 103 , 25631-25642.

Maricq, M.M., Xu, N., 2004. The effective density and fractal dimension of soot particles from premixed flames and motor vehicle exhaust. Aerosol Science 35 , 1251-1274.

Marinoni, A., Laj, P., Sellegri, K., Maihot, G., 2004. Cloud chemistry at the Puy de Dôme: variability and relationships with environmental factors. Atmospheric Chemistry and Physics 4, 715-728.

Marshak, A., Knyzaikhin, Y., Evans, K.D., Wiscombe, W.J., 2004. The "RED versus NIR" plane to retrieve broken-cloud optical depth from ground-based measurements. Journal of Atmospheric Science 61, 1911-1925.

Martin, R.V., Jacob, D.J., Logan, J.A., Bey, I., Yantosca, R.M., Staudt, A.C., Li, Q.B. Fiore, A.M., Duncan, B.N., Liu, H.Y., Ginoux, P., Thouret, V., 2002. Interpretation of TOMS observations of tropical tropospheric ozone with a global model and in situ observations. Journal of Geophysical Research - Atmospheres 107, 4351

Martin, R.V., Jacob, D.J., Chance, K., Kurosu, T.P., Palmer, P.I., Evans, M.J., 2003. Global inventory of nitrogen oxide emissions constrained by space-based observation of $\mathrm{NO}_{2}$ columns. Journal of Geophysical Research - Atmospheres 108, 4537.

Martin, R.V., Sioris, C.E., Chance, K., Ryerson, T.B., Bertram, T.H., Wooldridge, P.J. Cohen, R.C., Neuman, J.A., Swanson, A., Flocke, F.M., 2006. Evaluation of spacebased constraints on global nitrogen oxide emissions with regional aircraft measurements over and downwind of eastern North America. Journal of Geophysical Research - Atmospheres 111, D15308.

Martin, R.V., Sauvage, B., Folkins, I., Sioris, C.E., Boone, C., Bernath, P., Ziemke, J. 2007. Space-based constraints on the production of nitric oxide by lightning. Journal of Geophysical Research - Atmospheres 112, D09309.

Martinsson, B.G., Papaspiropoulos, G., Heintzenberg, J., Hermann, M., 2001. Fine mode particulate sulphur in the tropopause region measured from intercontinental flights (CARIBIC). Geophysical Research Letters 28, 1175-1178.

Martinsson, B.G., Nguyen, H.N., Brenninkmeijer, C.A.M., Zahn, A., Heintzenberg, J, Hermann, M., van Velthoven, P.F.J., 2005. Characteristics and origin of lowermost stratospheric aerosol at northern midlatitudes under volcanically quiescent conditions based on CARIBIC observations. Journal of Geophysical Research 110, D12201.

Mather, J., Stevens, P., Brune, W., 1997. $\mathrm{OH}$ and $\mathrm{HO}_{2}$ measurements using laserinduced fluorescence. Journal of Geophysical Research 102, 6427-6436.

Matsui, T., Kreidenweis, S.M., Pielke, R.A., Schichtel, B., Yu, H.B., Chin, M., Chu, D.A., Niyogi, D., 2004. Regional comparison and assimilation of GOCART and MODIS aerosol optical depth across the eastern US. Geophysical Research Letters 31, L21101.

Matsumoto, K., Kawamura, K., Uchida, M., Shibata, Y., Yoneda, M., 2001. Compound specific radiocarbon and $\delta^{13} \mathrm{C}$ measurements of fatty acids in a continental aerosol sample. Geophysical Research Letters 28, 4587-4590.

Matsumoto, J., Kosugi, N., Imai, H., Kajii, Y., 2005. Development of a measurement system for nitrate radical and dinitrogen pentoxide using a thermal conversion laser-induced fluorescence technique. Review of Scientific Instruments 76, 064101

Matthew, B.M., Middlebrook, A.M., Onasch, T.B., 2008. Collection efficiencies in an Aerodyne aerosol mass spectrometer as a function of particle phase for laboratory generated aerosols. Aerosol Science and Technology 42, 884-898.

Mattis, I., Müller, D., Ansmann, A., Wandinger, U., Preißler, J., Seifert, P., Tesche, M. 2008. Ten years of multiwavelength Raman lidar observations of free-tropospheric aerosol layers over Central Europe: geometrical properties and annual cycle. Journal of Geophysical Research 113, D20202.

McCabe, J.R., Savarino, J., Alexander, B., Gong, S., Thiemens, M.H., 2006. Isotopic constraints on non-photochemical sulfate production in the Arctic winter. Geophysical Research Letters 33, L05810.

McCormick, M.P., Winker, D.M., Browell, E.V., Coakley, J.A., Gardner, C.S., Hoff, R.M. Kent, G.S., Melfi, S.H., Menzies, R.T., Platt, C.M.R., Randall, D.A., Reagan, J.A., 1993. Scientific investigations planned for the Lidar-In-Space Technology Experiment (LITE). Bulletin of the American Meteorological Society 74, 205-214.

McFiggans, G., Coe, H., Burgess, R., Allan, J., Cubison, M., Alfarra, M.R., Saunders, R Saiz-Lopez, A., Plane, J.M.C., Wevill, D., Carpenter, L., Rickard, A.R., Monks, P.S. 2004. Direct evidence for coastal iodine particles from Laminaria macroalgae linkage to emissions of molecular iodine. Atmospheric Chemistry and Physics 4 , 701-713.

McGillen, M.R., Percival, C.J., Raventos-Duran, T., Sanchez-Reyna, G., Shallcross, D.E., 2006. Can topological indices be used to predict gas-phase rate coefficients of importance to tropospheric chemistry? Free radical abstraction reactions of alkanes. Atmospheric Environment 40, 2488-2500.

McGillen, M.R., Crosier, J., Percival, C.J., Sanchez-Reyna, G., Shallcross, D.E., 2006. Can topological indices be used to predict gas-phase rate coefficients of importance to tropospheric chemistry? Reactions of alkenes with $\mathrm{OH}, \mathrm{NO}_{3}$ and $\mathrm{O}_{3}$. Chemosphere 65, 2035-2044.

Mcllvin, M.R., Altabet, M.A., 2005. Chemical conversion of nitrate and nitrite to nitrous oxide for nitrogen and oxygen isotopic analysis in freshwater and seawater. Analytical Chemistry 77, 5589-5595.

McLaren, R., Salmon, R.A., Liggio, J., Hayden, K.L., Anlauf, K.G., Leaitch, W.R., 2004. Nighttime chemistry at a rural site in the Lower Fraser Valley. Atmospheric Environment 38, 5837-5848.

McMillan, W.W., Warner, J.X., Comer, M.M., Maddy, E., Chu, A., Sparling, L. Eloranta, E., Hoff, R., Sachse, G., Barnet, C., Razenkov, I., Wolf, W., 2008. AIRS views transport from 12 to 22 July 2004 Alaskan/Canadian fires: correlation of AIRS CO and MODIS AOD with forward trajectories and comparison of AIRS CO retrievals with DC-8 in situ measurements during INTEX-A/ICARTT. Journal of Geophysical Research - Atmospheres 113. doi:10.1029/2007JD009711.

McMurry, P.H., Wang, X., Park, K., Ehara, K., 2002. The relationship between mass and mobility for atmospheric particles: A new technique for measuring particle density. Aerosol Science and Technology 36, 227-238.

Meier, A., Notholt, J., 1996. Determination of the isotopic abundances of heavy $\mathrm{O}_{3}$ as observed in arctic ground-based FTIR spectra. Geophysical Research Letters 23 . 511-554.

Menut, L., 2003. Adjoint modeling for atmospheric pollution process sensitivity at regional scale. Journal of Geophysical Research 108.

Mertes, S., Schwarzenböck, A., Laj, P., Wobrock, W., Pichon, J.-M., Orsi, G. Heintzenberg, J., 2001. Changes of cloud microphysical properties during the transition from supercooled to mixed-phase conditions during CIME. Atmospheric Research SI 58, 267-294.

Mertes, S., Galgon, D., Schwirn, K., Nowak, A., Lehman, K., Massling, A., Wiedensohler, A., Wieprecht, W., 2005. Evolution of particle concentration and size distribution observed upwind, inside and downwind hill cap clouds at connected flow conditions during FEBUKO. Atmospheric Environment 39, $4233-4245$

Mertes, S., Verheggen, B., Walter, S., Connolly, P., Ebert, M., Schneider, J., Bower, K., Inerle-Hof, M., Cozic, J., Baltensperger, U., Weingartner, E., 2007. Counterflow virtual impactor based collection of small ice particles in mixed-phase clouds for the physico-chemical characterization of tropospheric ice nuclei: sampler description and first case study. Aerosol Science and Technology 41, 848-864.

Michalski, G. Savarino, J., Böhlke, J.K. Thiemens, M., 2002. Determination of the total oxygen isotopic composition of nitrate and the calibration of a $\delta^{17} \mathrm{O}$ nitrate reference material. Analytical Chemistry 74, 4989-4993.

Michalski, G., Kasem, M., Rech, J.A., Adieu, S., Showers, W.S., Genna, B., Thiemens, M.H., 2008. Uncertainties in the oxygen isotopic composition of barium sulfate induced by coprecipitation of nitrate. Rapid Communications in Mass Spectrometry 22.

Mihelcic, D., Ehhalt, D.H., Kulessa, G.F., Klomfass, J., Trainer, M., Schmidt, U., Rohrs, H., 1978. Measurement of free radicals in the atmosphere by matrix isolation and electron paramagnetic resonance. Geofisica pura e applicata 116.

Mihelcic, D., Klemp, D., Müsgen, P., Pätz, H.W., Volz-Thomas, A., 1993. Simultaneous measurements of peroxy and nitrate radicals at Schauinsland. Journal of Atmospheric Chemistry 16, 313-335.

Miller, B.R., Weiss, R.F., Salameh, P.K., Tanhua, T., Greally, B.R., Mühle, J., Simmonds, P.G., 2008. Medusa: a sample preconcentration and GC/MS detector system for in situ measurements of atmospheric trace halocarbons, hydrocarbons, and sulfur compounds. Analytical Chemistry 80, 1536-1545.

Mirme, A., Tamm, E., Mordas, G., Vana, M., Uin, J., Mirme, S., Bernotas, T., Laakso, L., Hirsikko, A., Kulmala, M., 2007. A wide-range multi-channel air ion spectrometer. Boreal Environmental Research 12, 247-264.

Mishchenko, M.I., Geogdzhayev, I.V., Liu, L., Ogren, J.A., Lacis, A.A., Rossow, W.B., Hovenier, J.W., Volten, H., Muñoz, O., 2003. Aerosol retrievals from AVHRR radiances: effects of particle nonsphericity and absorption and an updated long-term global climatology of aerosol properties. Journal of Quantitative Spectroscopy and Radiative Transfer 79, 953-972.

Mishchenko, M.I., Cairns, B., Kopp, G., Schueler, C.F., Fafaul, B.A., Hansen, J.E., Hooker, R.J., Itchkawich, T., Maring, H.B., Travis, L.D., 2007. Precise and accurate monitoring of terrestrial aerosols and total solar irradiance: introducing the Glory mission. Bulletin of the American Meteorological Society 88, 677-691.

Möhler, O., Büttner, S., Linke, C., Schnaiter, M., Saathoff, H., Stetzer, O., Wagner, R., Krämer, M., Mangold, A., Ebert, V., Schurath, U., 2005. Effect of sulfuric acid coating on heterogeneous ice nucleation by soot aerosol particles. Journal of Geophysical Research 110, D11210.

Möhler, O., Georgakopoulos, D., Morris, C., Benz, S., Ebert, V., Saathoff, H., Schnaiter, M., Wagner, F., 2008. Heterogeneous ice nucleation activity of Pseudomonas bacteria: new laboratory experiments at simulated cloud conditions. Biogeosciences 5, 1425-1435.

Möhler, O., Benz, S., Saathoff, H., Schnaiter, M., Wagner, R., Schneider, J., Walter, S. Ebert, M., Wagner, S., 2008. The effect of organic coating on the heterogeneous ice nucleation efficiency of mineral dust aerosols. Environmental Research Letters 3, 025007.

Möhler, O., DeMott, P.J., Stetzer, O., 2008c. The Fourth International Ice Nucleation Workshop ICIS-2007. In: The Proceedings of the International Conference on Clouds and Precipitation, Cancun, Mexico, July 7-11. 
Mona, L., Amodeo, A., Pandolfi, M., Pappalardo, G., 2006. Saharan dust intrusions in the Mediterranean area: three years of lidar measurements in Potenza. Journal of Geophysical Research 111, D16203. doi:10.1029/2005JD006569.

Monks, P.S., Granier, C., Fuzzi, S., Stohl, A., Williams, M., Akimoto, H., Amman, M., Baklanov, A., Baltensperger, U., Bey, I., Blake, N., Blake, R.S., Carslaw, K., Cooper, O.R., Dentener, F., Fowler, D., Fragkou, E., Frost, G., Generoso, S., Ginoux, P., Grewe, V., Guenther, A., Hansson, H.C., Henne, S., Hjorth, J., Hofzumahaus, A., Huntrieser, H., Isaksen, I.S.A., Jenkin, M.E., Kaiser, J., Kanakidou, M., Klimont, Z., Kulmala, M., Laj, P., Lawrence, M.G., Lee, J.D., Liousse, C., Maione, M., McFiggans, G., Metzger, A., Mieville, A., Moussiopoulos, N., Orlando, J.J., O’Dowd, C., Palmer, P.I., Parrish, D., Petzold, A., Platt, U., Pöschl, U., Prévôt, A.S.H., Reeves, C.E., Reiman, S., Rudich, Y., Sellegri, K., Steinbrecher, R., Simpson, D., ten Brink, H., Theloke, J., van der Werf, G., Vautard, R., Vestreng, V., Vlachokostas, Ch., vonGlasow, R. Atmospheric composition change - global and regional air quality, in this issue.

Moore, R.H., 2008. HTDMA analysis of multicomponent dicarboxylic acid aerosols with comparison to UNIFAC and ZSR. Journal of Geophysical Research 113, D04206.

Moore II, K.G., Clarke, A.D., Kapustin, V.N., McNaughton, C., Anderson, B.E. Winstead, E.L., Weber, R., Ma, Y., Lee, Y.N., Talbot, R., Dibb, J., Anderson, T. Doherty, S., Covert, D., Rogers, D., 2004. A comparison of similar aerosol measurements made on the NASA P3-B, DC-8, and NSF C-130 aircraft during TRACE-P and ACE-Asia. Journal of Geophysical Research 109, D15S15.

Moosmüller, H., Varma, R., Arnott, W.P., 2005. Cavity ring-down and cavityenhanced detection techniques for the measurement of aerosol extinction. Aerosol Science and Technology 39, 30-39.

Moretti, F., Tagliavini, E., Decesari, S., Facchini, M.C., Rinaldi, R., Fuzzi, S., 2008. NMR determination of total carbonyls and carboxyls: a tool for tracing the evolution of atmospheric oxidized organic aerosols. Environmental Science and Technology 42, 4844-4849.

Morin, S., Savarino, J., Frey, M.M., Yan, N., Bekki, S., Bottenheim, J.W., Martins, J.M.F., 2008. Tracing the origin and fate of $\mathrm{NO}_{\mathrm{x}}$ in the Arctic atmosphere using stable isotopes in nitrate. Science 322, 730-732.

Müller, D., Wandinger, U., Ansmann, A., 1999. Microphysical particle parameters from extinction and backscatter lidar data by inversion with regularization: theory. Applied Optics 38, 2346-2357.

Müller, D., Mattis, I., Ansmann, A., Wehner, B., Althausen, D., Wandinger, U., Dubovik, O., 2004. Comprehensive characterization of Arctic haze from combined observations with Raman lidar and Sun photometer. Journal of Geophysical Research 109, D13206.

Müller, D., Mattis, I., Wadinger, A., Ansmann, A, Althausen, D., Stohl, A, 2005. Raman lidar observations of aged Siberian and Canadian forest-fire smoke in the free troposphere over Germany in 2003: microphysical particle characterization. Journal of Geophysical Research 110, D17201.

Müller, D., Ansmann, A., Mattis, I., Tesche, M., Wadinger, A., Althausen, D., Pisani, G. 2007. Aerosol-type-dependent lidar-ratio observed with Raman lidar. Journal of Geophysical Research 112, D16202.

Müller, D., Mattis, I., Ansmann, A., Wadinger, A., Althausen, D., 2007. Raman lidar for monitoring of aerosol pollution in the free troposphere. In: Kim, Y.J., Platt, U. (Eds.), Advanced Environmental Monitoring. Springer, New York, pp. 155-166.

Müller, L., Reinning, M.C., Warneke, J., Hoffmann, T., 2008. Unambiguous identification of esters as oligomers in secondary organic aerosol formed from cyclohexene and cyclohexene/alpha-pinene ozonolysis. Atmospheric Chemistry and Physics 8, 1423-1433.

Murphy, D.M., 2007. The design of single particle laser mass spectrometers. Mass Spectrometry Reviews 26, 150-165.

Murphy, D.M., Thomson, D.S., Mahoney, M.J., 1998. In situ measurements of organics, meteoritic material, mercury, and other elements in aerosols at 5 to 19 kilometers. Science 282, 1664-1669.

Murphy, D.M., Middlebrook, A.M., Warshawsky, M., 2003. Cluster analysis of data from the particle analysis by laser mass spectrometry (PALMS) instrument. Aerosol Science and Technology 37, 382-391.

Murphy, D.M., Cziczo, D.J., Froyd, K.D., Hudson, P.K., Matthew, B.M., Middlebrook, A.M., Peltier, R.E., Sullivan, A., Thomson, D.S., Weber, R.J., 2006. Single-particle mass spectrometry of tropospheric aerosol particles. Journal of Geophysical Research 111, D23S32.

Murphy, D.M., Hudson, P.K., Thompson, D.S., Sheridan, P.J., Wilson, J.C., 2006. Observations of mercury-containing aerosols. Environmental Science and Technology 40, 3163-3167.

Murphy, D.M., Cziczo, D.J., Hudson, P.K., Thompson, D.S., 2007. Carbonaceous material in aerosol particles in the lower stratosphere and tropopause region. Journal of Geophysical Research 112, D04203.

Murphy, D.M., Thomson, D.S., 1995. Laser ionization mass-spectroscopy of single aerosol-particles. Aerosol Science and Technology 22, 237-249.

Murray, B.J., 2008. Inhibition of ice crystallisation in highly viscous aqueous organic acid droplets. Atmospheric Chemistry and Physics 8, 5423-5433.

Murray, B.J., Bertram, A.K., 2008. Inhibition of solute crystallisation in aqueous $\mathrm{H}^{+}-$ $\mathrm{NH}_{4}^{+}-\mathrm{SO}_{4}^{2}-\mathrm{H}_{2} \mathrm{O}$ droplets. Physical Chemistry and Chemical Physics $10,3287-$ 3301.

Nakajima, T., Higurashi, A., Kawamoto, K., Penner, J., 2001. A possible correlation between satellite-derived cloud and aerosol microphysical parameters. Geophysical Research Letters 28, 1171-1174.

Nappo, C.J., Caneill, J.Y., Furman, R.W., Gifford, F.A., Kaimal, J.C., Kramer, M.L., Lockhart, T.J., Pendergast, M.M., Pielke, R.A., Randerson, D., Shreffler, J.H.,
Wyngaard, J.C., 1982. The workshop on the representativeness of meteorological-observations, June 1981, Boulder, Colorado. Bulletin of the American Meteorological Society 63, 761-764.

Nash, D.G., Baer, T., Johnston, M.V., 2006. Aerosol mass spectrometry: an introductory review. International Journal of Mass Spectrometry 258, 2-12.

Nassar, R., Logan, J.A., Worden, H.M., Megretskaia, I.A., Bowman, K.W., Osterman, G.B., Thompson, A.M., Tarasick, D.W., Austin, S., Claude, H. Dubey, M.K., Hocking, W.K., Johnson, B.J., Joseph, E., Merrill, J., Morris, G.A., Newchurch, M., Oltmans, S.J., Posny, F., Schmidlin, F.J., Vomel, H. Whiteman, D.N., Witte, J.C., 2008. Validation of tropospheric emission spectrometer (TES) nadir ozone profiles using ozonesonde measurements. Journal of Geophysical Research - Atmospheres 113, D15S17.

NDACC (Network for the Detection of Atmospheric Composition Change), 2008. NDACC Validation Protocol. http://www.ndsc.ncep.noaa.gov/organize/ protocols/prot_val/ (accessed 15.10.08).

Neefs, E., De Mazière, M., Scolas, F., Hermans, C., Hawat, T., 2007. BARCOS an automation and remote control system for atmospheric observations with a Bruker interferometer. Review of Scientific Instruments 78, 035109.

Neff, J.C., Holland, E.A., Dentener, F.J., McDowell, W.H., Russell, K.M., 2002. The origin, composition and rates of organic nitrogen deposition: a missing piece of the nitrogen cycle? Biogeochemistry 57, 99-136.

Nemitz, E., Jimenez, J.L., Huffman, J.A., Ulbrich, I.M., Canagaratna, M.R. Worsnop, D.R., Guenther, A.B., 2008. An eddy-covariance system for the measurement of surface/atmosphere exchange fluxes of submicron aerosol chemical species - first application above an urban area. Aerosol Science and Technology 42, 636-657.

Nguyen, H.N., Martinsson, B.G., Wagner, J.B., Carlemalm, E., Ebert, M., Weinbruch, S. Brenninkmeijer, C.A.M., Heintzenberg, J., Hermann, M., Schuck, T., van Velthoven, P.F.J., Zahn, A., 2008. Chemical composition and morphology of individual aerosol particles from a CARIBIC flight at $10 \mathrm{~km}$ altitude between $50^{\circ} \mathrm{N}$ and $30^{\circ} \mathrm{S}$. Journal of Geophysical Research 113, D23209.

Nisbet, E., 2007. Earth monitoring: Cinderella science. Nature 450, 789-790.

Northway, M.J., Jayne, J.T., Toohey, D.W., Canagaratna, M.R., Trimborn, A., Akiyama, K.I., Shimono, A., Jimenez, J.L., DeCarlo, P.F., Wilson, K.R., Worsnop, D.R., 2007. Demonstration of a VUV lamp photoionization source for improved organic speciation in an aerosol mass spectrometer. Aerosol Science and Technology 41, 828-839.

Notholt, J., Kuang, Z., Rinsland, C.P., Toon, G.C., Rex, M., Jones, N., Albrecht, T., Deckelmann, H., Krieg, J., Weinzierl, C., Bingemer, H., Weller, R., Schrems, O. 2003. Enhanced upper tropical tropospheric COS: impact on the stratospheric aerosol layer. Science 300, 307-310.

Notholt, J., Lehmann, R., 2003. The moon as light source for atmospheric trace gas observations: measurement technique and analysis method. Journal of Quantitative Spectroscopy and Radiative Transfer 76, 435-445.

O'Keefe, A., Deacon, D.A.G., 1988. Cavity ring-down optical spectrometer for absorption-measurements using pulsed laser sources. Review of Scientific Instruments 59, 2544-2551.

O'Neill, N.T., Eck, T.F., Smirnov, A., Holben, B.N., Thulasiraman, S., 2003. Spectra discrimination of coarse and fine mode optical depth. Journal of Geophysical Research 108, 4559.

Oltmans, S.J., Lefohn, A.S., Harris, J.M., Galbally, I., Scheel, H.E., Bodeker, G., Brunke, E., Claude, H., Tarasick, D., Johnson, B.J., Simmonds, P., Shadwick, D. Anlauf, K., Hayden, K., Schmidlin, F., Fujimoto, T., Akagi, K., Meyer, C., Nichol, S. Davies, J., Redondas, A., Cuevas, E., 2006. Long-term changes in tropospheric ozone. Atmospheric Environment 40, 3156-3173.

Omar, A.H., Won, J.-G., Winker, D.M., Yoon, S.-C., Dubovik, O., McCormick, M.P. 2005. Development of global aerosol models using cluster analysis of Aerosol Robotic Network (AERONET) measurements. Journal of Geophysical Research 110 , D10S14.

Ordonez, C., Mathis, H., Furger, M., Henne, S., Huglin, C., Staehelin, J., Prevot, A.S.H., 2005. Changes of daily surface ozone maxima in Switzerland in all seasons from 1992 to 2002 and discussion of summer 2003. Atmospheric Chemistry and Physics 5, 1187-1203.

Orlando, J.J., 2007. The atmospheric oxidation of diethyl ether: chemistry of the $\mathrm{C}_{2} \mathrm{H}_{5}-\mathrm{O}-\mathrm{CH}(\mathrm{O}) \mathrm{CH}_{3}$ radical between 218 and $335 \mathrm{~K}$. Physical Chemistry and Chemical Physics 9, 4189-4199.

Orlando, J.J., Tyndall, G.S., Betterton, E.A., Lowry, J., Stegall, S.T., 2005. Atmospheric chemistry of hydrazoic acid $\left(\mathrm{HN}_{3}\right)$ : UV absorption spectrum, $\mathrm{HO}$ reaction rate, and reactions of the $\mathrm{N}_{3}$ radical. Environmental Science and Technology 39, $1632-1640$

Orsini, D.A., Ma, Y.L., Sullivan, A., Sierau, B., Baumann, K., Weber, R.J., 2003. Refinements to the particle-into-liquid sampler (PILS) for ground and airborne measurements of water soluble aerosol composition. Atmospheric Environment 37, 1243-1259.

Ortega, J., Helmig, D., Guenther, A., Harley, P., Pressley, S., Vogel, C., 2007. Flux estimates and $\mathrm{OH}$ reaction potential of reactive biogenic volatile organic compounds (BVOCs) from a mixed northern hardwood forest. Atmospheric Environment 41, 5479-5495.

Ortega, J., Helmig, D., Daly, R.W., Tanner, D.M., Guenther, A., Herrick, J.D., 2008 Approaches for quantifying reactive and low-volatility biogenic organic compound emissions by vegetation enclosure techniques - part B: applications. Chemosphere 72, 365-380. doi:10.1016/j.chemosphere.2008.02.054.

Ortega, J., Helmig, D., 2008. Approaches for quantifying reactive and low-volatility biogenic organic compound emissions by vegetation enclosure techniques part A. Chemosphere 72, 343-364. doi:10.1016/j.chemosphere.2007.11.020. 
Oshchepkov, S., Isaka, H., Gayet, J.-F., Sinyuk, A., Auriol, F., Havemann, S., 2000 Microphysical properties of mixed-phase \& ice clouds retrieved from in situ airborne "polar nephelometer" measurements. Geophysical Research Letters 27, 209-212.

Osterman, G.B., Kulawik, S.S., Worden, H.M., Richards, N.A.D., Fisher, B.M. Eldering, A., Shephard, M.W., Froidevaux, L., Labow, G., Luo, M., Herman, R.L Bowman, K.W., Thompson, A.M., 2008. Validation of tropospheric emission spectrometer (TES) measurements of the total, stratospheric, and tropospheric column abundance of ozone. Journal of Geophysical Research - Atmospheres 113, D15S16.

Paatero, P., 1997. Least squares formulation of robust non-negative factor analysis. Chemometrics and Intelligent Laboratory Systems 37, 23-35.

Pappalardo, G., Amodeo, A. Mona, L. Pandolfi, M., Pergola, N., Cuomo, V., 2004. Raman lidar observations of aerosol emitted during the 2002 Etna eruption. Geophysics Research Letters 31, L05120. doi:10.1029/2003GL019073.

Papaspiropoulos, G., Martinson, B.G., Zahn, A., Brenninkmeijer, C.A.M. Hermann, M., Heintzenberg, J., Fischer, H., van Velthoven, P.F.J., 2002. Aeroso elemental concentrations in the tropopause region from intercontinental flights with the Civil Aircraft for Regular Investigation of the Atmosphere Based on an Instrument Container (CARIBIC) platform. Journal of Geophysica Research 107, 4671.

Park, K., 2003. Relationship between particle mass and mobility for diesel exhaust particles. Environmental Science and Technology 37, 577-583.

Park, M., Randel, W.J., Emmons, L.K., Bernath, P.F., Walker, K.A., Boone, C.D., 2008. Chemical isolation in the Asian monsoon anticyclone observed in Atmospheric Chemistry Experiment (ACE-FTS) data. Atmospheric Chemistry and Physics 8, 757-764.

Parrington, M., Jones, D.B.A., Bowman, K.W., Horowitz, L.W., Thompson, A.M., Tarasick, D.W., Witte, J.C., 2008. Estimating the summertime tropospheric ozone distribution over North America through assimilation of observations from the tropospheric emission spectrometer. Journal of Geophysical Research - Atmospheres 113.

Parrish, D.D., Dunlea, E.J., Atlas, E.L., Schauffler, S., Donnelly, S., Stroud, V. Goldstein, A.H., Millet, D.B., McKay, M., Jaffe, D.A., Price, H.U., Hess, P.G. Flocke, F., Roberts, J.M., 2004. Changes in the photochemical environment of the temperate North Pacific troposphere in response to increased Asian emissions. Journal of Geophysical Research - Atmospheres 109, D23S18.

Parsons, 2006. Crystallization of aqueous inorganic-malonic acid particles: nucleation rates, dependence on size, and dependence on the ammonium-to-sulfate ratio. Journal of Physical Chemistry - Atmospheres, 8108-8115. 2006

Paulsen, D., Dommen, J., Kalberer, M., Prévôt, A.S.H., Richter, R., Sax, M. Steinbacher, M., Weingartner, E., Baltensperger, U., 2005. Secondary organic aerosol formation by irradiation of $1,3,5$ trimethylbenzene- $\mathrm{NO}_{x}-\mathrm{H}_{2} \mathrm{O}$ in a new reaction chamber for atmospheric chemistry and physics. Environmental Science and Technology 39, 2668-2678.

Paulsen, D., Weingartner, E., Rami Alfarra, M., Baltensperger, U., 2006. Volatility measurements of photochemically and nebulizer-generated organic aerosol particles. Journal of Aerosol Science 37, 1025-1051.

Peng, C., Man, N.C., Chan, C.K., 2001. The hygroscopic properties of dicarboxylic and multifunctional acids: measurements and UNIFAC predictions. Environmenta Science and Technology 35, 4495-4501.

Perner, D., Platt, U., 1979. Detection of nitrous acid in the atmosphere by differentia optical absorption. Geophysical Research Letters 6, 917-920.

Petersen, A.K., Warneke, T., Lawrence, M.G., Notholt, J., Schrems, O., 2008. First ground-based FTIR observations of the seasonal variation of carbon monoxide in the tropics. Geophysical Research Letters 35, L03813.

Petron, G., Granier, C., Khattatov, B., Yudin, V., Lamarque, J.F., Emmons, L., Gille, J. Edwards, D.P., 2004. Monthly CO surface sources inventory based on the 20002001 MOPITT satellite data. Geophysical Research Letters 31.

Pettersson, A., Lovejoy, E.R., Brock, C.A., Brown, S.S., Ravishankara, A.R., 2004. Measurement of aerosol optical extinction at $532 \mathrm{~nm}$ with pulsed cavity ring down spectroscopy. Journal of Aerosol Science 35, 995-1011.

Peuch, A., Thepaut, J.N., Pailleux, J., 2000. Dynamical impact of total-ozone observations in a four-dimensional variational assimilation. Quarterly Journal of the Royal Meteorological Society 126, 1641-1659.

Pfister, G., Hess, P.G., Emmons, L.K., Lamarque, J.F., Wiedinmyer, C., Edwards, D.P. Petron, G., Gille, J.C., Sachse, G.W., 2005. Quantifying CO emissions from the 2004 Alaskan wildfires using MOPITT CO data. Geophysical Research Letters 32 L11809.

Phares, D.J., Rhoads, K.P., Wexler, A.S., Kane, D.B., Johnston, M.V., 2001. Application of the ART-2a algorithm to laser ablation aerosol mass spectrometry of particle standards. Analytical Chemistry 73, 2338-2344.

Pio, C.A., Legrand, M., Oliveira, T., Afonso, J., Santos, C., Caseiro, A., Fialho, P., Puxbaum, H., Sanchez-Ochoa, A., Kasper-Giebl, A., Gelencsér, A., Preunkert, S. Schock, M., 2007. Climatology of aerosol composition (organic versus inorganic) at nonurban sites on a west-east transect across Europe. Journal of Geophysical Research 112, D23S02.

Plass-Dülmer, C., Schmidbauer, N., Slemr, J., Slemr, F., D’Souza, H., 2006. European hydrocarbon intercomparison experiment AMOHA part 4: Canister sampling of ambient air. Journal of Geophysical Research 111, D04306.

Platt, U., Perner, D., Winer, A., Harris, G., Pitts Jr., J., 1980. Detection of $\mathrm{NO}_{3}$ in the polluted troposphere by differential optical-absorption. Geophysical Research Letters 7, 89-92.

Platt, U., Perner, D., Schröder, J., Kessler, C., Toennissen, A., 1981. The diurnal-variation of $\mathrm{NO}_{3}$. Journal of Geophysical Research 86, 1965-1970.
Platt, U., Perner, D., 1980. Direct measurements of atmospheric $\mathrm{CH}_{2} \mathrm{O}, \mathrm{HNO}_{2}, \mathrm{O}_{3}$, $\mathrm{NO}_{2}$, and $\mathrm{SO}_{2}$ by differential optical absorption in the near UV. Journal of Geophysical Research 85, 7453-7458.

Platt, U., Stutz, J., 2006. Differential Optical Absorption Spectroscopy (DOAS), Principle and Applications. Springer Verlag, Heidelberg, Germany.

Pope, F.D., Smith, C.A., Davis, P.R., Shallcross, D.E., Ashfold, M.N.R., Orr-Ewing, A.J., 2005. The photochemistry of formaldehyde under tropospheric conditions. Faraday Discussions 130, 59-72.

Poulson, S.R., 2005. The effect of sulfate- $\delta^{18} \mathrm{O}$ upon on-line sulfate- $\delta^{34} \mathrm{~S}$ analysis, and implications for measurements of $\delta^{33} \mathrm{~S}$ and $\delta^{33} \mathrm{~S}$. Rapid Communications in Mass Spectrometry 19, 105-107.

Pradier, S., Attie, J.L., Chong, M., Escobar, J., Peuch, V.-H., Lamarque, J.F., Khattatov, B., Edwards, D., 2006. Evaluation of 2001 springtime CO transport over West Africa using MOPITT CO measurements assimilated in a global chemistry transport model. Tellus B 58, 163-176.

Prata, A.J., Bernardo, C., 2007. Retrieval of volcanic $\mathrm{SO}_{2}$ column abundance from atmospheric infrared sounder data. Journal of Geophysical Research - Atmospheres 112, D20204.

Prata, A.J., Kerkmann, J., 2007. Simultaneous retrieval of volcanic ash and $\mathrm{SO}_{2}$ using MSG-SEVIRI measurements. Geophysical Research Letters 34, L05813.

Prenni, A.J., DeMott, P.J., Kreidenweis, S.M., 2003. Water uptake of internally mixed particles containing ammonium sulfate and dicarboxylic acids. Atmospheric Environment 37, 4243-4251.

Prenni, A.J., Petters, M.D., Kreidenweis, S.M., DeMott, P.J., Ziemann, P.J., 2007. Cloud droplet activation of secondary organic aerosol. Journal of Geophysical Research 112, 12.

Pugnaghi, S., Gangale, G., Corradini, S., Buongiorno, M.F., 2006. Mt. Etna sulfur dioxide flux monitoring using ASTER-TIR data and atmospheric observations. Journal of Volcanology and Geothermal Research 152, 74-90.

Rader, D.J., McMurry, P.H., 1986. Application of the tandem differential mobility analyzer to studies of droplet growth or evaporation. Journal of Aerosol Science 17, 771-787.

Rajakumar, B., Gierczak, T., Flad, J.E., Ravishankara, A.R., Burkholder, J.B., 2008. The $\mathrm{CH}_{3} \mathrm{CO}$ quantum yield in the $248 \mathrm{~nm}$ photolysis of acetone, methyl ethyl ketone, and biacetyl. Journal of Photochemistry and Photobiology A 199, 336-344.

Rappenglück, B., Apel, E., Bauerfeind, M., Bottenheim, J., Brickell, P., Cavolka, P., Cech, J., Gatti, L., Hakola, H., Honzak, J., Junek, R., Martin, D., Noone, C., PlassDülmer, C., Travers, D., Wang, D., 2006. The first VOC intercomparison exercise within the Global Atmosphere Watch (GAW). Atmospheric Environment 40, 7508-7527.

Rasch, P.., Collins, W.D., Eaton, B.E., 2001. Understanding the Indian Ocean Experiment (INDOEX) aerosol distributions with an aerosol assimilation. Journal of Geophysical Research - Atmospheres 106, 7337-7355.

Rattigan, O.V., Hogrefe, O., Felton, H.D., Schwab, J.J., Roychowdhury, U.K., Husain, L., Dutkiewicz, V.A., Demerjian, K.L., 2006. Multi-year urban and rural semicontinuous PM2.5 sulfate and nitrate measurements in New York State: evaluation and comparison with filter based measurements. Atmospheric Environment 40, S192-S205.

Rebotier, T.P., Prather, K.A., 2007. Aerosol time-of-flight mass spectrometry data analysis: a benchmark of clustering algorithms. Analytica Chimica Acta 585, 38-54.

Reinhardt, A., Emmenegger, C., Gerrits, B., Panse, C., Dommen, J., Baltensperger, U., Zenobi, R., Kalberer, M., 2007. Ultrahigh mass resolution and accurate mass measurements as a tool to characterize oligomers in secondary organic aerosols. Analytical Chemistry 79, 4074-4082.

Remer, L.A., Kaufman, Y.J., Tanré, D., Mattoo, S., Chu, D.A., Martins, J.V., Li, R.R., Ichoku, C., Levy, R.C., Kleidman, R.G., Eck, T.F., Vermote, E., Holben, B.N., 2005. The MODIS aerosol algorithm, products and validation. Journal of Atmospheric Science 62, 947-972.

Ren, X., Edwards, G.D., Cantrell, C.A., Lesher, R.L., Metcalf, A.R., Shirley, T., Brune, W.H., 2003. Intercomparison of peroxy radical measurements at a rural site using laser-induced fluorescence and Peroxy Radical Chemical Ionization Mass Spectrometer (PerCIMS) techniques. Journal of Geophysical Research 108, 4605.

Revesz, K., Böhlke, J.K., Yoshinari, T., 1997. Determination of $\delta^{18} \mathrm{O}$ and $\delta^{15} \mathrm{~N}$ in nitrate. Analytical Chemistry 69, 4375-4380.

Richter, A., Wittrock, F., Eisinger, M., Burrows, J.P., 1998. GOME observations of tropospheric $\mathrm{BrO}$ in Northern Hemispheric spring and summer 1997. Geophysical Research Letters 25, 2683-2686.

Richter, A., Eyring, V., Burrows, J.P., Bovensmann, H., Lauer, A., Sierk, B., Crutzen, P.J., 2004. Satellite measurements of $\mathrm{NO}_{2}$ from international shipping emissions. Geophysical Research Letters 31, L23110.

Richter, A., Burrows, J.P., Nuss, H., Granier, C., Niemeier, U., 2005. Increase in tropospheric nitrogen dioxide over China observed from space. Nature 437, 129-132.

Richter, A., Wagner, T., 2008. Validation results for the Atmospheric Chemistry Experiment (ACE). In: Richter, A., Wagner, T. (Eds.), ACPD Special Issue. Atmospheric Chemistry and Physics Discussions, vols. 7-8.

Riemer, N., Vogel, H., Vogel, B., Schell, B., Ackermann, I., Kessler, C., Hass, H., 2003. Impact of the heterogeneous hydrolysis of $\mathrm{N}_{2} \mathrm{O}_{5}$ on chemistry and nitrate formation in the lower troposphere under photosmog conditions. Journal of Geophysical Research 108, 4144

Riipinen, I., Svenningsson, B., Bilde, M., Gaman, A., Lehtinen, K.E.J., Kulmala, M. 2006. A method for determining thermophysical properties of organic material in aqueous solutions: Succinic acid. Atmospheric Research 82, 579-590. 
Riipinen, I., Koponen, K.I., Frank, G.P., Hyvaerinen, A.P., Vanhanen, J., Lihavainen, H., Lehtinen, K.E.J., Bilde, M., Kulmala, M., 2007. Adipic and malonic acid aqueous solutions: surface tensions and saturation vapor pressures. Journal of Physical Chemistry 111, 12995-13002.

Rinsland, C.P., Dufour, G., Boone, C.D., Bernath, P.F., Chiou, L., 2005. Atmospheric Chemistry Experiment (ACE) measurements of elevated Southern Hemisphere upper tropospheric $\mathrm{CO}, \mathrm{C}_{2} \mathrm{H}_{6}, \mathrm{HCN}$, and $\mathrm{C}_{2} \mathrm{H}_{2}$ mixing ratios from biomass burning emissions and long-range transport. Geophysical Research Letters 32, L20803.

Rinsland, C.P., Boone, C.D., Bernath, P.F., Mahieu, E., Zander, R., Dufour, G., Clerbaux, C., Turquety, S., Chiou, L., McConnell, J.C., Neary, L., Kaminski, J.W., 2006. First space-based observations of formic acid $(\mathrm{HCOOH})$ : Atmospheric Chemistry Experiment austral spring 2004 and 2005 Southern Hemisphere tropical-mid-latitude upper tropospheric measurements. Geophysical Research Letters 33, L23804.

Rinsland, C.P., Coheur, P.F., Herbin, H., Clerbaux, C., Boone, C., Bernath, P., Chiou, L.S., 2007. Detection of elevated tropospheric hydrogen peroxide $\left(\mathrm{H}_{2} \mathrm{O}_{2}\right)$ mixing ratios in Atmospheric Chemistry Experiment (ACE) subtropical infrared solar occultation spectra. Journal of Quantitative Spectroscopy and Radiative Transfer 107, 340-348.

Rinsland, C.P., Dufour, G., Boone, C.D., Bernath, P.F., Chiou, L., Coheur, P.F., Turquety, S., Clerbaux, C., 2007. Satellite boreal measurements over Alaska and Canada during June-July 2004: simultaneous measurements of upper tropospheric $\mathrm{CO}, \mathrm{C}_{2} \mathrm{H}_{6}, \mathrm{HCN}, \mathrm{CH}_{3} \mathrm{Cl}, \mathrm{CH}_{4}, \mathrm{C}_{2} \mathrm{H}_{2}, \mathrm{CH}_{3} \mathrm{OH}, \mathrm{HCOOH}$, OCS, and $\mathrm{SF}_{6}$ mixing ratios. Global Biogeochemical Cycles 21, GB3008.

Riziq, A.A., Erlick, C., Dinar, E., Rudich, Y., 2007. Optical properties of absorbing and non-absorbing aerosols retrieved by cavity ring down (CRD) spectroscopy. Atmospheric Chemistry and Physics 7, 1523-1536.

Riziq, A.A., Trainic, M., Erlick, C., Serge, E., Rudich, Y., 2008. Extinction efficiencies of coated absorbing aerosols measured by cavity ring down aerosol spectrometry. Atmospheric Chemistry and Physics 8, 1823-1833.

Roberts, P., Hallett, J., 1968. A laboratory study of the ice nucleating properties of some mineral particulates. Quarterly Journal of the Royal Meteorological Society $94,25-34$.

Roberts, G., Nenes, A., 2005. A continuous-flow streamwise thermal-gradient CCN chamber for atmospheric measurements. Aerosol Science and Technology 39, 206-221.

Robinson, A.L., Donahue, N.M., Shrivastava, M.K., Weitkamp, E.A., Sage, A.M., Grieshop, A.P., Lane, T.E., Pierce, J.R., Pandis, S.N., 2007. Rethinking organic aerosols: semivolatile emissions and photochemical aging. Science 315, 12591262.

Rogers, D.C., 1988. Development of a continuous flow thermal gradient diffusion chamber for ice nucleation studies. Atmospheric Research 22, 149-181.

Rogers, D.C., DeMott, P.J., Kreidenweis, S.M., Chen, Y.L., 2001. A continuous-flow diffusion chamber for airborne measurements of ice nuclei. Journal of Atmospheric and Oceanic Technology 18, 725-741.

Rohrer, F., Berresheim, H., 2006. Strong correlation between levels of tropospheric hydroxyl radicals and solar ultraviolet radiation. Nature 442, 184-187.

Rudolph, J., 2007. Gas chromatography-isotope ratio mass spectrometry. In: Koppmann, R. (Ed.), Volatile Organic Compounds in the Atmosphere. Blackwell Publishing, Oxford, pp. 388-466.

Rudolph, J., Czuba, E., 2000. On the use of isotopic composition measurements of volatile organic compounds to determine the "photochemical age" of an air mass. Geophysical Research Letters 27, 3865-3868.

Rudolph, J., Lowe, D.C., Martin, R.J., Clarkson, T.S., 1997. A novel method for the compound specific determination of $\delta^{13} \mathrm{C}$ in volatile organic compounds at ppt levels in ambient air. Geophysical Research Letters 24, 659-662.

Russchenberg, H., Bosveld, F., Swart, D., Brink, H., deLeeuw, G., Uijlenhout, R., Abresse, B., Boers, R., Apituley, A., 2005. Ground based atmospheric remote sensing in the Netherlands: European outlook. IEICE Transactions on Communications, E86.

Sadanaga, Y., Yoshino, A., Kato, S., Yoshioka, A., Watanabe, K., Miyakawa, Y., Hayashi, I., Ichikawa, M., Matsumoto, J., Nishiyama, A., Kanaya, Y., Kajii, Y., 2004. The importance of $\mathrm{NO}_{2}$ and volatile organic compounds in the urban air from the viewpoint of the $\mathrm{OH}$ reactivity. Geophysical Research Letters 31, L08102.

Sadanaga, Y., Yoshino, A., Kato, S., Kajii, Y., 2005. Measurements of OH reactivity and photochemical ozone production in the urban atmosphere. Environmental Science and Technology 33, 8847-8852.

Saito, A., Murakami, M., 2008. Measurement of natural ice nuclei by continuous flow thermal diffusion chamber type ice nucleus counter. In: International Conference on Clouds and Precipitation, Cancun, Mexico.

Saiz-Lopez, A., Chance, K., Liu, X., Kurosu, T.P., Sander, S.P., 2007. First observations of iodine oxide from space. Geophysical Research Letters 34.

Saiz-Lopez, A., Mahajan, A.S., Salmon, R.A., Bauguitte, S.J.B., Jones, A.E., Roscoe, H.K., Plane, J.M.C., 2007. Boundary layer halogens in coastal Antarctica. Science 317, 348-351.

Sakurai, H., Park, K., McMurry, P.H., Zarling, D.D., Kittelson, D.B., Ziemann, P.J., 2003. Size-dependent mixing characteristics of volatile and nonvolatile components in diesel exhaust aerosols. Environmental Science and Technology 37, 5487-5495.

Salam, A., Lohmann, U., Crenna, B., Lesins, G., Klages, P., Rogers, D., Irani, R. MacGillivray, A., Coffin, M., 2006. Ice nucleation studies of mineral dust particles with a new continuous flow diffusion chamber. Aerosol Science and Technology 40, 134-143.

Salt, K., Noble, C.A., Prather, K.A., 1996. Aerodynamic particle sizing versus light scattering intensity measurement as methods for real time particle sizing coupled with time-of-flight mass spectrometry. Analytical Chemistry 68 ,
$230-234$.

Salt, K., Noble, C.A., Prather, K.A., 1998. Coupling two-step laser desorption ionization with time-of-flight mass spectrometry. Analytical Chemistry 9 , 230-234.

Sannigrahi, P., Sullivan, A.P., Weber, R.J., Ingall, E.D., 2006. Characterization of watersoluble organic carbon in urban atmospheric aerosols using solid-state ${ }^{13} \mathrm{C}$ NMR spectroscopy. Environmental Science and Technology 40, 666-672.

Sanusi, A.A., Norman, A.-L., Burridge, C., Wadleigh, M., Tang W.-, W., 2006. Determination of the $S$ isotope composition of methanesulfonic acid. Analytical Chemistry 78, 4964-4968.

Sappey, A.D., Hill, E.S., Settersten, T., Linne, M.A., 1998. Fixed frequency cavity ringdown diagnostic for atmospheric particulate matter. Optics Letters 23.

Sarigiannis, D.A., Saisana, M., 2008. Multi-objective optimization of air quality monitoring. Environmental Monitoring and Assessment 136, 87-99.

Sassen, K., 2000. Lidar backscatter depolarization technique for cloud and aerosol research. In: Mishchenko, M.L., Hovenier, J.W., Travis, L.D. (Eds.), Light Scattering by Nonspherical Particles: Theory, Measurements, and Geophysical Applications. Academic Press, pp. 393-416.

Sassen, K. Wang, Z., Liu, D., 2008. Global distribution of cirrus clouds from CloudSat/Cloud-Aersol Lidar and Infrared Pathfinder Satellite Observations (CALIPSO) measurements. Journal of Geophysical Research 113, D00A12.

Sassen, K., Mace, G.G., 2002. Ground based remote sensing of cirrus clouds. In: Lynch, D., Sassen, K., Starr, D.O.C., Stephens, G.L. (Eds.), Cirrus. Oxford University Press, pp. 168-195.

Sauvage, B., Thouret, V., Cammas, J.-P., Brioude, J., Nédélec, P., Mari, C., 2007. Meridional ozone gradients in the African upper troposphere. Geophysical Research Letters 34, L03817. doi:10.1029/2006GL028542.

Savarino, J., Alexander, B., Darmohusodo, V., Thiemens, M.H., 2001. Sulfur and oxygen isotope analysis of sulfate at micromole levels using a pyrolysis technique in a continuous flow system. Analytical Chemistry 73, 4457-4462.

Schade, G.W., Custer, T.G., 2004. OVOC emissions from agricultural soil in northern Germany during the 2003 European heat wave. Atmospheric Environment 38, 6105-6114.

Schaller, R.C., Fukuta, N., 1979. Ice nucleation by aerosol particles: experimental studies using a wedge-shaped ice thermal diffusion chamber. Journal of Atmospheric Science 36, 1788-1802.

Schaub, D., Boersma, K.F., Kaiser, J.W., Weiss, A.K., Folini, D., Eskes, H.J., Buchmann, B., 2006. Comparison of GOME tropospheric $\mathrm{NO}_{2}$ columns with $\mathrm{NO}_{2}$ profiles deduced from ground-based in situ measurements. Atmospheric Chemistry and Physics 6, 3211-3229.

Scherer, J.J., Paul, J.B., O’Keefe, A., Saykally, R.J., 1997. Cavity ringdown laser absorption spectroscopy: history, development, and application to pulsed molecular beams. Chemical Reviews 97, 25-51.

Schilt, S., Thévenaz, L., Niklès, M., Emmenegger, L., Hüglin, C., 2004. Ammonia monitoring at trace level using photoacoustic spectroscopy in industrial and environmental applications. Spectrochimica Acta A 60, 3259-3268.

Schlosser, E., Bohn, B., Brauers, T., Dorn, H.P., Fuchs, H., Haseler, R., Hofzumahaus, A. Holland, F., Rohrer, F., Rupp, L.O., Siese, M., Tillmann, R., Wahner, A., 2007. Intercomparison of two hydroxyl radical measurement techniques at the atmosphere simulation chamber SAPHIR. Journal of Atmospheric Chemistry 56, 187-205.

Schlosser, E., Brauers, T., Dorn, H.-P., Fuchs, H., Häseler, R., Hofzumahaus, A. Holland, F., Wahner, A., Kanaya, Y., Kajii, Y., Miyamoto, K., Nishida, S. Watanabe, K., Yoshino, A., Kubistin, D., Martinez, M., Rudolf, M., Harder, H., Berresheim, H., Elste, T., Plass-Dülmer, C., Stange, G., Schurath, U., 2009. Forma blind intercomparison of $\mathrm{OH}$ measurements: results from the international campaign HOxComp. Technical Note. Atmospheric Chemistry and Physics Discussions 9, 14081-14139.

Schlussel, P., Hultberg, T.H., Phillips, P.L., August, T., Calbet, X., 2005. The operational IASI level 2 processor. In: Burrows, J.P., Eichmann, K.U. (Eds.), Atmospheric Remote Sensing: Earth's Surface, Troposphere, Stratosphere and Mesosphere I, pp. 982-988

Schmid, O., Artaxo, P., Arnott, W.P., Chand, D., Gatti, L.V., Frank, G.P., Hoffer, A. Schnaiter, M., Andreae, M.O., 2006. Spectral light absorption by ambient aerosols influenced by biomass burning in the Amazon Basin. I: comparison and field calibration of absorption measurement techniques. Atmospheric Chemistry and Physics 6, 3443-3462.

Schneider, M. Blumenstock, T. Hase, F, Höpfner, M., Cuevas, $E$, Redondas, A Sancho, J.M., 2005. Ozone profiles and total column amounts derived at Izaña, Tenerife Island, from FTIR solar absorption spectra, and its validation by an intercomparison to ECC-sonde and Brewer spectrometer measurements. Journal of Quantitative Spectroscopy and Radiative Transfer 91, 245-274

Schneider, M., Hase, F., Blumenstock, T., 2006. Water vapour profiles by groundbased FTIR spectroscopy: study for an optimised retrieval and its validation. Atmospheric Chemistry and Physics 6, 811-830.

Schneider, M., Hase, F., Blumenstock, T., 2006. Ground-based remote sensing of $\mathrm{HDO} / \mathrm{H}_{2} \mathrm{O}$ ratio profiles: introduction and validation of an innovative retrieval approach. Atmospheric Chemistry and Physics 6, 4705-4722.

Schneider, M., Hase, F., Blumenstock, T., Redondas, A., Cuevas, E., 2008. Quality assessment of $\mathrm{O}_{3}$ profiles measured by a state-of-the-art ground-based FTIR observing system. Atmospheric Chemistry and Physics 8, 5579-5588.

Schneider, M., Redondas, A., Hase, F., Guirado, C., Blumenstock, T., Cuevas, E., 2008 Comparison of ground-based Brewer and FTIR total column $\mathrm{O}_{3}$ monitoring techniques. Atmospheric Chemistry and Physics 8, 5535-5550. 
Schneider, M., Sussmann, R., Notholt, J. Fourier transform infrared spectrometry. In: Sounding Systems for Atmospheric Water Vapor Monitoring. International Space Science Institute, in press.

Schneider, M., Hase, F., 2008. Technical note: recipe for monitoring of total ozone with a precision of around 1 DU applying mid-infrared solar absorption spectra. Atmospheric Chemistry and Physics 8, 63-71.

Schneider, M., Hase, F., 2009. Reviewing the development of a ground-based FTIR water vapour profile analysis. Atmospheric Measurement Techniques Discussions 2, 1221-1246.

Schoeberl, M.R., Ziemke, J.R., Bojkov, B., Livesey, N., Duncan, B., Strahan, S Froidevaux, L., Kulawik, S., Bhartia, P.K., Chandra, S., Levelt, P.F., Witte, J.C. Thompson, A.M., Cuevas, E., Redondas, A., Tarasick, D.W., Davies, J., Bodeker, G. Hansen, G., Johnson, B.J., Oltmans, S.J. Vomel, H., Allaart, M., Kelder, H., Newchurch, M., Godin-Beekmann, S., Ancellet, G., Claude, H., Andersen, S.B., Kyro, E., Parrondos, M., Yela, M., Zablocki, G., Moore, D., Dier, H., von der Gathen, P., Viatte, P., Stubi, R., Calpini, B., Skrivankova, P., Dorokhov, V., de Backer, H., Schmidlin, F.J., Coetzee, G., Fujiwara, M., Thouret, V., Posny, F., Morris, G., Merrill, J., Leong, C.P., Koenig-Langlo, G., Joseph, E., 2007. A trajectory-based estimate of the tropospheric ozone column using the residua method. Journal of Geophysical Research - Atmospheres 112, D24S49.

Schönhardt, A., Richter, A., Wittrock, F., Kirk, H., Oetjen, H., Roscoe, H.K. Burrows, J.P., 2008. Observations of iodine monoxide columns from satellite. Atmospheric Chemistry and Physics 8, 637-653.

Schuster, G.L., Dubovik, O., Holben, B.N., Clothiaux, E.E., 2005. Inferring black carbon content and specific absorption from Aerosol Robotic Network (AERONET) aerosol retrievals. Journal of Geophysical Research 110, D10S17.

Seakins, P.W., Robertson, S.H., 2007. Synergies between experimental and theoretical studies of gas phase reactions. Physical Chemistry and Chemical Physics 9, 4053-4054.

Seebaugh, R., Wilson, J.C., 1999. High-volume low-turbulence inlet for aeroso sampling from aircraft. Annual Report to the National Science Foundation Division of Atmospheric Chemistry.

Seinfeld, J.H., Pandis, S.N., 1998. Atmospheric Chemistry and Physics: from Air Pollution to Climate Change. John Wiley \& Sons, Inc., New York.

Sekiguchi, M., Nakajima, T., Suzuki, T., Kawamoto, K., Higurashi, A., Rosenfeld, D., Sano, I., Mukai, S., 2003. A study of the direct and indirect effects of aerosols using global satellite data sets of aerosol and cloud parameters. Journal of Geophysical Research 108, 4699.

Sellegri, K., Laj, P., Dupuy, R., Legrand, M., Preunkert, S., Putaud J.-, P., 2003. Sizedependent scavenging efficiencies of multicomponent atmospheric aerosols in clouds. Journal of Geophysical Research 108, 4334.

Sellegri, K., Villani, P., Picard, D., Dupuy, R., O’Dowd, C., Laj, P., 2008. Role of the volatile fraction of submicron marine aerosol on its hygroscopic properties. Atmospheric Research 90 (2-4), 272-277.

Sellevag, S.R., Nielsen, C.J., Sovde, O.A., Myrhe, G., Sundet, J.K., Stordal, F., Isaksen, I.S., 2004. Atmospheric gas-phase degradation and global warming potentials of 2-fluoroethanol, 2,2-difluoroethanol, and 2,2,2-trifluoroethanol. Atmospheric Environment 38, 6725-6735.

Senten, C., De Mazière, M., Dils, B., Hermans, C., Kruglanski, M., Neefs, E., Scolas, F. Vandaele, A.C., Vanhaelewyn, G., Vigouroux, C., Carleer, M., Coheur, P.F., Fally, S. Barret, B., Baray, J.L., Delmas, R., Leveau, J., Metzger, J.M., Mahieu, E., Boone, C. Walker, K.A., Bernath, P.F., Strong, K., 2008. Technical note: ground-based FTIR measurements at Ile de La Réunion: observations, error analysis and comparisons with satellite data. Atmospheric Chemistry and Physics 8, 3483-3508.

Sgro, L.A., De la Mora, J.F., 2004. A simple turbulent mixing CNC for charged particle detection down to $1.2 \mathrm{~nm}$. Aerosol Science and Technology 38, 1-11.

Shindell, D.T., Faluvegi, G., Emmons, L.K., 2005. Inferring carbon monoxide pollution changes from space-based observations. Journal of Geophysical Research Atmospheres 110, D23303.

Shupe, M.D., Daniel, J.S., De Boer, G., Eloranta, E.W., Kollias, P., Luke, E., Long, C.N. Turner, D.D., Verlinde, J., 2008. A focus on mixed-phase clouds: the status of ground-based observational methods. Bulletin of the American Meteorologica Society 89 (10), 1549-1562. doi:10.1175/2008BAMS2378.1

Sigman, D.M., Casciotti, K.L., Andreani, M., Barford, C., Galanter, M., Böhlke, J.K., 2001. A bacterial method for the nitrogen isotopic analysis of nitrate in marine and fresh waters. Analytical Chemistry 73, 4145-4153.

Silva, S.R., Kendall, C., Wilkinson, D.H., Ziegler, A.C., Chang, C.C.Y., Avanzino, R.J., 2000. A new method for collection of nitrate from fresh water and nitrogen and oxygen isotope ratios. Journal of Hydrology 228, 22-36.

Simpson, W.R., 2003. Continuous wave cavity ring-down spectroscopy applied to in situ detection of dinitrogen pentoxide $\left(\mathrm{N}_{2} \mathrm{O}_{5}\right)$. Review of Scientific Instruments 74, 3442-3452.

Simpson, D., Yttri, K., Klimont, Z., Kupiainen, K., Caseiro, A., Gelencser, A., Pio, C. Legrand, M., 2007. Modeling carbonaceous aerosol over Europe. Analysis of the CARBOSOL and EMEP EC/OC campaigns. Journal of Geophysical Research 112 D23S14. doi:10.1029/2006JD008158.

Simpson, W.R., von Glasow, R., Riedel, K., Anderson, P., Ariya, P., Bottenheim, J. Burrows, J., Carpenter, L.J., Friess, U., Goodsite, M.E., Heard, D., Hutterli, M. Jacobi, H.W., Kaleschke, L., Neff, B., Plane, J., Platt, U., Richter, A., Roscoe, H., Sander, R., Shepson, P., Sodeau, J., Steffen, A., Wagner, T., Wolff, E., 2007. Halogens and their role in polar boundary-layer ozone depletion. Atmospheric Chemistry and Physics 7, 4375-4418.

Singh, H.B., Salas, L.J., Chatfield, R.B., Czech, E., Fried, A., Walega, J., Evans, M.J. Field, B.D., Jacob, D.J., Blake, D., Heikes, B., Talbot, R., Sachse, G., Crawford, J.H., Avery, M.A., Sandholm, S., Fuelberg, H., 2004. Analysis of the atmospheric distribution, sources, and sinks of oxygenated volatile organic chemicals based on measurements over the Pacific during TRACE-P. Journal of Geophysical Research 109, D15S07.

Sinha, V., Williams, J., Crowley, J.N., Lelieveld, J., 2008. The comparative reactivity method - a new tool to measure total $\mathrm{OH}$ Reactivity in ambient air. Atmospheric Chemistry and Physics 8, 2213-2227.

Sinha, B.W., Hoppe, P., Huth, J., Foley, S., Andreae, M.O., 2008. Sulfur isotope analyses of individual aerosol particles in the urban aerosol at a central European site (Mainz, Germany). Atmospheric Chemistry and Physics 8, 7217-7238.

Sinreich, R., Volkamer, R., Filsinger, F., Frieß, U., Kern, C., Platt, U., Sebastián, O., Wagner, T., 2007. MAX-DOAS detection of glyoxal during ICARTT 2004. Atmospheric Chemistry and Physics 7, 1293-1303.

Sipilä, M., Lehtipalo, K., Kulmala, M., Petäjä, T., Junninen, H., Aalto, P.P. Manninen, H.E., Kyro, E.-M., Asmi, E., Riipinen, I., Curtius, J., Kurten, A., Borrmann, S., O'Dowd, C.D., 2008. Applicability of condensation particle counters to measure atmospheric clusters. Atmospheric Chemistry and Physics $8,4049-4060$.

Sivakumaran, V., Crowley, J.N., 2003. Reaction between $\mathrm{OH}$ and $\mathrm{CH}_{3} \mathrm{CHO}$. Part 2. Temperature dependent rate coefficients (201-348 K). Physical Chemistry and Chemical Physics 5, 106-111.

Sive, B.C., Zhou, Y., Troop, D., Wang, Y., Little, W.C., Wingenter, O.W., Russo, R.S., Varner, R.K., Talbot, R., 2005. Development of a cryogen-free concentration system for measurements of volatile organic compounds. Analytical Chemistry 77, 6989-6998.

Skillas, G., Künzel, S., Burtscher, H., Baltensperger, U., Siegmann, K., 1998. High fractal-like dimension of diesel soot agglomerates. Journal of Aerosol Science 29, 411-419.

Sommariva, R., Pilling, M.J., Bloss, W.J., Heard, D.E., Lee, J.D., Fleming, Z.L., Monks, P.S., Plane, J.M.C., Saiz-Lopez, A., Ball, S.M., Bitter, M., Jones, R.L., Brough, N., Penkett, S.A., Hopkins, J.R., Lewis, A.C., Read, K.A., 2007. Night-time radical chemistry during the NAMBLEX campaign. Atmospheric Chemistry and Physics 7, 587-598.

Sorooshian, A., Murphy, S.M., Hersey, S., Gates, H., Padro, L.T., Nenes, A., Brechtel, F.J., Jonsson, H., Flagan, R.C., Seinfeld, J.H., 2008. Comprehensive airborne characterization of aerosol from a major bovine source. Atmospheric Chemistry and Physics 8, 5489-5520.

Spangenberg, T., Köhler, S., Hansmann, B., Wachsmuth, U., Abel, B., 2004. Lowtemperature reactions of $\mathrm{OH}$ radicals with propene and isoprene in pulsed laval nozzle expansions. Journal of Physical Chemistry A 108, 7527-7534.

Spindler, C., Abo Riziq, A., Rudich, Y., 2007. Retrieval of aerosol complex refractive index by combining cavity ring down aerosol spectrometer measurement with full size distribution information. Aerosol Science and Technology 41, 1011-1017.

Spirig, C., Neftel, A., Ammann, A., Dommen, J., Grabmer, W., Thielmann, A., Schaub, A., Beauchamp, J., Wisthaler, A., Hansel, A., 2005. Eddy covariance flux measurements of biogenic VOCs during ECHO 2003 using proton transfer reaction mass spectrometry. Atmospheric Chemistry and Physics 5, 465-481.

Spracklen, D.V., Arnold, S.R., Sciare, J., Carslaw, K.S., Pio, C., 2008. Globally significant oceanic source of organic carbon aerosol. Geophysical Research Letters 35, L12811.

Stanier, C.O., Pathak, R.K., Pandis, S.N., 2007. Measurements of the volatility of aerosols from a-pinene ozonolysis. Environmental Science and Technology 41, 2756-2763.

Stark, H., Brown, S.S., Burkholder, J.B., Aldener, M., Riffault, V., Gierczak, T., Ravishankara, A.R., 2008. Overtone dissociation of peroxynitric acid $\left(\mathrm{HO}_{2} \mathrm{NO}_{2}\right)$ : absorption cross sections and photolysis products. Journal of Physical Chemistry $112,9296-9303$.

Steck, T., Glatthor, N., von Clarmann, T., Fischer, H., Flaud, J.M., Funke, B., Grabowski, U., Hopfner, M., Kellmann, S., Linden, A., Perrin, A., Stiller, G.P., 2008. Retrieval of global upper tropospheric and stratospheric formaldehyde $\left(\mathrm{H}_{2} \mathrm{CO}\right)$ distributions from high-resolution MIPAS-Envisat spectra. Atmospheric Chemistry and Physics 8, 463-470.

Steinbacher, M., Dommen, J., Ammann, C., Spirig, C., Neftel, A., Prevot, A.S.H., 2004 Performance characteristics of a proton-transfer-reaction mass spectrometer (PTR-MS) derived from laboratory and field measurements. International Journal of Mass Spectrometry 239, 117-128.

Stephens, G.L., Tsay, S., Stackhouse, P.W., Flatau, P.J., 1990. The relevance of the microphysical and radiative properties of cirrus clouds to climate and climatic feedback. Journal of Atmospheric Science 47, 1742-1753.

Stephens, G.L., Vane, D.G., Boain, R.J., Mace, G.G., Sassen, K., Wang, Z. Illingworth, A.J., O'Connor, E.J., Rossow, W.B., Durden, S.L., Miller, S.D., Austin, R.T., Benedetti, A., Mitrescu, C., The CloudSat Science Team, 2002. The CloudSat mission and the A-Train: a new dimension of space-based observations of clouds and precipitation. Bulletin of the American Meteorological Society $83,1771-1790$

Stetzer, O., Baschek, B., Lüönd, F., Lohmann, U., 2008. The Zurich Ice Nucleation Chamber (ZINC) - a new instrument to investigate atmospheric ice formation. Aerosol Science and Technology 42, 64-74.

Still, T.J., Al-Haider, S., Seakins, P.W., Sommariva, R., Stanton, J.C., Mills, G., Penkett, S.A., 2006. Ambient formaldehyde measurements made at a remote marine boundary layer site during the NAMBLEX campaign - a comparison of data from chromatographic and modified Hantzsch techniques. Atmospheric Chemistry and Physics 6, 2711-2726.

Stoffelen, A., Marseille, G.-J., Andersson, E., Tan, D.G.H., 2005. The atmospheric dynamics mission for global wind field measurement. Bulletin of the American Meteorological Society 86 
Stohl, A., Berg, T., Burkhart, J.F., Fjaeraa, A.M., Forster, C., Herber, A., Hov, O., Lunder, C., McMillan, W.W., Oltmans, S., Shiobara, M., Simpson, D., Solberg, S., Stebel, K., Strom, J., Torseth, K., Treffeisen, R., Virkkunen, K., Yttri, K.E., 2007. Arctic smoke - record high air pollution levels in the European Arctic due to agricultural fires in Eastern Europe in spring 2006. Atmospheric Chemistry and Physics 7, 511-534.

Stohl, A., Forster, C., Huntrieser, H., Mannstein, H., McMillan, W.W., Petzold, A., Schlager, H., Weinzierl, B., 2007. Aircraft measurements over Europe of an air pollution plume from Southeast Asia - aerosol and chemical characterization. Atmospheric Chemistry and Physics 7, 913-937.

Stolzenburg, M.R., Hering, S.V., 2000. Method for the automated measurement of fine particle nitrate in the atmosphere. Environmental Science and Technology 34, 907-914.

Stone, D., Rowley, D.M., 2005. Kinetics of the gas phase $\mathrm{HO}_{2}$ self-reaction: effects of temperature, pressure, water and methanol vapours. Physical Chemistry and Chemical Physics 7, 2156-2163.

Stratmann, F., Kiselev, A., Wurzler, S., Wendisch, M., Heintzenberg, J., Charlson, R.J., Diehl, K., Wex, H., Schmidt, S., 2004. Laboratory studies and numerical simulations of cloud droplet formation under realistic supersaturation conditions. Journal of Atmospheric and Oceanic Technology 21, 876-887.

Strawa, A.W., Castaneda, R., Owano, T., Baer, D.S., Paldus, B.A., 2003. The measurement of aerosol optical properties using continuous wave cavity ring-down techniques. Journal of Atmospheric and Oceanic Technology 20, 454-465.

Stutz, J., Alicke, B., Ackermann, R., Geyer, A., White, A., Williams, E., 2004. Vertical profiles of $\mathrm{NO}_{3}, \mathrm{~N}_{2} \mathrm{O}_{5}, \mathrm{O}_{3}$, and $\mathrm{NO}_{\mathrm{x}}$ in the nocturnal boundary layer: 1 . Observations during the Texas Air Quality Study 2000. Journal of Geophysical Research 109, D12306.

Suess, D.T., Prather, K.A., 1999. Mass spectrometry of aerosols. Chemical Reviews 99, 3007-3035.

Sugimoto, N., Shimizu, A., Matsui, I., Itsushi, U., Arao, K., Chen, Y., Zhao, S., Zhou, J., Lee C.-, H., 2005. Study of dust transport using a network of continuously operated polarization lidars. Water, Air, and Soil Pollution: Focus 5.

Sullivan, A.P., Weber, R.J., Clements, A.L., Turner, J.R., Bae, M.S., Schauer, J.J., 2004. A method for on-line measurement of water-soluble organic carbon in ambient aerosol particles: results from an urban site. Geophysical Research Letters 31, L13105.

Sullivan, R.C., Prather, K.A., 2005. Recent advances in our understanding of atmospheric chemistry and climate made possible by on-line aerosol analysis instrumentation. Analytical Chemistry 77, 3861-3885.

Surratt, J.D., Murphy, S.M., Kroll, J.H., Ng, N.L., Hildebrandt, L., Sorooshian, A., Szmigielski, R, Vermeylen, R. Maenhaut, W. Claeys, M. Flagan, R.C. Seinfeld, J.H., 2006. Chemical composition of secondary organic aerosol formed from the photooxidation of isoprene. Journal of Physical Chemistry - Atmospheres $110,9665-9690$.

Surratt, J.D., Kroll, J.H., Kleindienst, T.E., Edney, E.O., Claeys, M., Sorooshian, A., Ng, N.L., Offenberg, J.H., Lewandowski, M., Jaoui, M., Flagan, R.C., Seinfeld, J.H., 2007. Evidence for organosulfates in secondary organic aerosol. Environmental Science and Technology 41, 517-527.

Surratt, J.D., Gomez-Gonzalez, Y., Chan, A.W.H., Vermeylen, R., Shahgholi, M., Kleindienst, T.E., Edney, E.O., Offenberg, J.H., Lewandowski, M., Jaoui, M., Maenhaut, W., Claeys, M., Flagan, R.C., Seinfeld, J.H., 2008. Organosulfate formation in biogenic secondary organic aerosol. Journal of Physical Chemistry - Atmospheres 112, 8345-8378.

Sussmann, R., Buchwitz, M., 2005. Initial validation of ENVISAT/SCIAMACHY columnar CO by FTIR profile retrievals at the Ground-Truthing Station Zugspitze. Atmospheric Chemistry and Physics 5, 1497-1503.

Sussmann, R., Stremme, W., Buchwitz, M., de Beek, R., 2005. Validation of ENVISAT/ SCIAMACHY columnar methane by solar FTIR spectrometry at the GroundTruthing Station Zugspitze. Atmospheric Chemistry and Physics 5, 2419-2429.

Swietlicki, E., Hansson, H.-C., Hämeri, K., Svenningsson, B., Massling, A., McFiggans, G., McMurry, P., Petäjä, T., Tunved, P., Gysel, M., Topping, D., Weingartner, E., Baltensperger, U., Rissler, J., Wiedensohler, A., Kulmala, M. 2008. Hygroscopic properties of submicrometer atmospheric aerosol particles measured with H-TDMA instruments in various environments - a review. Tellus 60B, 432-469.

Syage, J.A., Hanning-Lee, M.A., Hanold, K.A., 2000. A man-portable, photoionization time-of-flight mass spectrometer. Field Analytical Chemistry and Technology 4, 204-215.

Szidat, S., Jenk, T.M., Synal, H.A., Kalberer, M., Wacker, L., Hajdas, I., Kasper-Giebl, A. Baltensperger, U., 2006. Contributions of fossil fuel, biomass-burning, and biogenic emissions to carbonaceous aerosols in Zurich as traced by ${ }^{14} \mathrm{C}$. Journal of Geophysical Research 111, D07206.

Szidat, S., Prévôt, A.S.H., Sandradewi, J., Alfarra, M.R., Synal, H.A., Wacker, L., Baltensperger, U., 2007. Dominant impact of residential wood burning on particulate matter in alpine valleys during winter. Geophysical Research Letters 34. doi:10.1029/2006GL028325.

Szidat, S., Ruff, M., Wacker, L., Synal, H.-A., Hallquist, M., Shannigrahi, A.S., Yttri, K.E., Dye, C., Simpson, D., 2009. Fossil and non-fossil sources of organic carbon (OC) and elemental carbon (EC) in Göteborg, Sweden. Atmospheric Chemistry and Physics 9, 1521-1535.

Szmigielski, R., Surratt, J.D., Vermeylen, R., Szmigielska, K., Kroll, J.H., Ng, N.L., Murphy, S.M., Sorooshian, A., Seinfeld, J.H., Claeys, M., 2007. Characterization of 2-methylglyceric acid oligomers in secondary organic aerosol formed from the photooxidation of isoprene using trimethylsilylation and gas chromatography/ ion trap mass spectrometry. Journal of Mass Spectrometry 42, 101-116.
Tajiri, T., Yamashita, K., Murakami, M., Orikasa, N., Saito, A., Nagai, T., Sakai, T., Ishimoto, H., 2008. Laboratory experiments of mixed-phase cloud formation. In International Conference on Clouds and Precipitation, Cancun, Mexico.

Tan, D., 2006. LIF Detection of trace gas species. In: Heard, D. (Ed.), Free Radicals in the Troposphere, ACCENT Report 6.2006, Sep. 2006. ACCENT Secretariat, Urbino, Italy, pp. 111-113.

Tanner, D., Helming, J., Hueber, J., Goldan, P., 2006. Gas chromatography system for the automated, unattended, and cryogen-free monitoring of $C_{2}$ to $C_{6}$ nonmethane hydrocarbons in the remote troposphere. Journal of Chromatography A $1111,76-88$.

Tatarov, B., Sugimoto, N., 2005. Estimation of quartz concentration in the tropospheric mineral aerosols using combined Raman and high-spectral-resolution lidars. Optics Letters 30, 3407-3409.

Tatarov, B., Sugimoto, N., Matusi, I., 2006. Lidar determination of quartz concentration in the tropospheric mineral aerosols - methodology and first results, in Laser remote sensing of the atmosphere. In: Selected Papers of the 23rd International Laser Radar Conference, Nara, Japan.

ten Brink, H., Otjes, R., Jongejan, P., Slanina, S., 2007. An instrument for semicontinuous monitoring of the size-distribution of nitrate, ammonium, sulphate and chloride in aerosol. Atmospheric Environment 41, 2768-2779.

Textor, C., Schulz, M., Guibert, S., Kinne, S., Balkanski, Y., Bauer, S., Berntsen, T., Berglen, T., Boucher, O., Chin, M., Dentener, F., Diehl, T., Easter, R., Feichter, H. Fillmore, D., Ghan, S., Ginoux, P., Gong, S., Grini, A., Hendricks, J., Horowitz, L. Huang, P., Isaksen, I., Iversen, T., Kloster, S., Koch, D., Kirkevåg, A Kristjansson, J.E., Krol, M., Lauer, A., Lamarque, J.F., Liu, X., Montanaro, V. Myhre, G., Penner, J., Pitari, G., Reddy, S., Seland, Ø., Stier, P., Takemura, T., Tie, X 2006. Analysis and quantification of the diversities of aerosol life cycles within AeroCom. Atmospheric Chemistry and Physics 6, 1777-1813.

Thompson, J.E., Smith, B.W., Winefordner, J.D., 2002. Monitoring atmospheric particulate matter through cavity ring-down spectroscopy. Analytical Chemistry $74,1962-1967$.

Theys, N., Van Roozendael, M., Hendrick, F., Fayt, C., Hermans, C., Baray, J.-L., Goutail, F., Pommereau, J.-P., De Mazière, M., 2007. Retrieval of stratospheric and tropospheric BrO columns from multi-axis DOAS measurements at Reunion Island $\left(21^{\circ} \mathrm{S}, 56^{\circ} \mathrm{E}\right)$. Atmospheric Chemistry and Physics 7, 4733-4749.

Theys, N., Van Roozendael, M., Dils, B., Hendrick, F., Hao, N., De Mazière, M., 2009. First satellite detection of volcanic bromine monoxide emission after the Kasatochi eruption. Geophysics Research Letters 36, L03809. doi:10.1029/ 2008GL036552.

Thiebaud, J., Crunaire, S., Fittschen, C., 2007. Measurements of line strengths in the $2 v_{1}$ band of the $\mathrm{HO}_{2}$ radical using laser photolysis/continuous wave cavity ringdown spectroscopy (cw-CRDS). Journal of Physical Chemistry 11, 6959-6966.

Thompson, A.M., Doddridge, B.G., Witte, J.C., Hudson, R.D., Luke, W.T., Johnston, J.E., Johnston, B.J., Oltmans, S.J., Weller, R., 2000. A tropical Atlantic paradox: shipboard and satellite views of a tropospheric ozone maximum and wave-one in January-February 1999. Geophysical Research Letters 27, 3317-3320.

Thompson, A.M., Witte, J.C., Oltmans, S.J., Schmidlin, F.J., 2004. Shadoz - a tropical ozonesonde-radiosonde network for the atmospheric community. Bulletin of the American Meteorological Society 85, 1549.

Thouret, V., Cammas, J.-P., Sauvage, B., Athier, G., R. Z., Nédélec, P., Simon, P. Karcher, F., 2006. Tropopause referenced ozone climatology and inter-annual variability (1994-2003) from the MOZAIC programme. Atmospheric Chemistry and Physics 8, 2133-2150.

Tobias, H.J., Kooiman, P.M., Docherty, K.S., Ziemann, P.J., 2000. Real-time chemical analysis of organic aerosols using a thermal desorption particle beam mass spectrometer. Aerosol Science and Technology 33, 170-190.

Todd, M.C., Washington, R., Martins, V., Dubovik, O., Lizcano, G., M'Bainayerl, S., Engelstaedter, S., 2007. Mineral dust emission from the Bodèlè depression, Chad during BoDEx. Journal of Geophysical Research 112, D06207.

Tolocka, M.P., Jang, M., Ginter, J.M., Cox, F.J., Kamens, R.M., Johnston, M.V., 2004 Formation of oligomers in secondary organic aerosol. Environmental Science and Technology 38, 1428-1434

Tressol, M., Ordonez, C., Zbinden, R., Thouret, V., Mari, C., Nédélec, P., Cammas, J.-P., Smit, H., Patz, H.-W., Volz-Thomas, A., 2008. Air pollution during the 2003 European heat wave as seen by MOZAIC airliners. Atmospheric Chemistry and Physics 8, 2133-2150.

Treuel, L., Schulze, S., Leisner, T., Zellner, R., 2008. Deliquescence behaviour of single levitated ternary salt/carboxylic acid/water microdroplets. Faraday Discussions 137, 267-278.

Turner, D., 2007. Improved ground-based liquid water path retrievals using a combined infrared and microwave approach. Journal of Geophysical Research 112, D15204.

Turner, D.D., Ferrare, R.A., Brasseur, L.A.H., Feltz, W.F., 2002. Automated retrievals of water vapor and aerosol profiles from an operational Raman lidar. Journal of Atmospheric and Oceanic Technology 19, 37-50.

Turquety, S., Hadji-Lazaro, J., Clerbaux, C., 2002. First satellite ozone distributions retrieved from nadir high-resolution infrared spectra. Geophysical Research Letters 29.

Turquety, S., Logan, J.A., Jacob, D.J., Hudman, R.C., Leung, F.Y., Heald, C.L, Yantosca, R.M., Wu, S.L., Emmons, L.K., Edwards, D.P., Sachse, G.W., 2007. Inventory of boreal fire emissions for North America in 2004: importance of peat burning and pyroconvective injection. Journal of Geophysical Research Atmospheres 112, D12S03.

Turquety, S., Clerbaux, C., Law, K., Coheur, P.-F., Cozic, A., Szopa, S., Hauglustaine, D.A., Hadji-Lazaro, J., Gloudemans, A.M.S., Schrijver, H. 
Boone, C.D., Bernath, P.F., Edwards, D.P., 2008. CO emission and export from Asia: an analysis combining complementary satellite measurements (MOPITT, SCIAMACHY and ACE-FTS) with global modeling. Atmospheric Chemistry and Physics 8, 1680-7316.

Urai, M., 2004. Sulfur dioxide flux estimation from volcanoes using advanced spaceborne thermal emission and reflection radiometer - a case study of Miyakejima volcano, Japan. Journal of Volcanology and Geothermal Research 134, 1-13.

Van der A, R.J., Peters, D.H.M.U., Eskes, H., Boersma, K.F., Van Roozendael, M., De Smedt, I., Kelder, H.M., 2006. Detection of the trend and seasonal variation in tropospheric $\mathrm{NO}_{2}$ over China. Journal of Geophysical Research 111, D12317.

Van der A, R.J., Eskes, H.J., Boersma, K.F., van Noije, T.P.C., Van Roozendael, M., De Smedt, I., Peters, D.H.M.U., Meijer, E.W., 2008. Trends, seasonal variability and dominant $\mathrm{NO}_{\mathrm{x}}$ source derived from a ten year record of $\mathrm{NO}_{2}$ measured from space. Journal of Geophysical Research 113, D04302.

VanReken, T.M., Ng, N.L., Flagan, R.C., Seinfeld, J.H., 2005. Cloud condensation nucleus activation properties of biogenic secondary organic aerosol. Journal of Geophysical Research 110, D07206.

VanReken, T.M., Greenberg, J.P., Harley, P.C., Guenther, A.B., Smith, J.N., 2006. Direct measurement of particle formation and growth from the oxidation of biogenic emissions. Atmospheric Chemistry and Physics 6, 4403-4413.

Varutbangkul, V., Brechtel, F.J., Bahreini, R., Ng, N.L., Keywood, M.D., Kroll, J.H. Flagan, R.C., Seinfeld, J.H., Lee, A., Goldstein, A.H., 2006. Hygroscopicity of secondary organic aerosols formed by oxidation of cycloalkenes, monoterpenes, sesquiterpenes, and related compounds. Atmospheric Chemistry and Physics 6, 2367-2388.

Veefkind, J.P., de Leeuw, G., Durkee, P.A., 1998. Retrieval of aerosol optical depth over land using two-angle view satellite radiometry during TARFOX. Geophysical Research Letters 25, 3135-3138.

Velazco, V., Notholt, J., Warneke, T., Lawrence, M., Bremer, H., Drummond, J. Schulz, A., Krieg, J., Schrems, O., 2005. Latitude and altitude variability of carbon monoxide in the Atlantic detected from ship-borne Fourier transform spectrometry, model, and satellite data. Journal of Geophysical Research 110 D09306.

Venzac, H., Sellegri, K., Laj, P., Villani, P., Bonasoni, P., Marinoni, A., Cristofanelli, P., Calzolari, F., Fuzzi, S., Decesari, S., Facchini, M.-C., Vuillermoz, E., Verza, G.P. 2008. High frequency new particle formation in the Himalayas. Proceedings National Academy of Science 105, 15666-15671.

Verheggen, B., Cozic, J., Weingartner, E., Bower, K., Mertes, S., Connolly, P., Gallagher, M., Flynn, M., Choularton, T., Baltensperger, U., 2007. Aerosol partitioning between the interstitial and the condensed phase in mixed-phase clouds. Journal of Geophysical Research 112, D23202.

Verheggen, B., Cozic, J., Weingartner, E., Bower, K., Mertes, S., Connolly, P., Gallagher, M., Flynn, M., Choularton, T., Baltensperger, U., 2007. Aerosol activation in mixed phase clouds at the high alpine site Jungfraujoch. Journal of Geophysical Research 112. doi:10.1029/2007JD008714.

Veselovskii, I., Kolgotin, A., Griaznov, V., Müller, D., Wandinger, U., Whiteman, D.N., 2002. Inversion with regularization for the retrieval of tropospheric aeroso parameters from multiwavelength lidar sounding. Applied Optics 41, 36853699.

Veselovskii, I., Kolgotin, A., Griaznov, V., Müller, D., Franke, K., Whiteman, D.N 2004. Inversion of multiwavelength Raman lidar data for retrieval of bimodal aerosol size distribution. Applied Optics 43, 1180-1195.

Veselovskii, I., Kolgotin, A., Griaznov, V., Müller, D., Whiteman, D.N., 2005. Information content of multiwavelength lidar data with respect to microphysical particle properties derived from eigenvalue analysis. Applied Optics 44, 5292-5303.

Vigouroux, C., De Mazière, M., Demoulin, P., Servais, C., Hase, F., Blumenstock, T. Kramer, I., Schneider, M., Melllqvist, J., Strandberg, A., Velazco, V., Notholt, J., Sussman, R., Stremme, W., Rockmann, A., Gardiner, T., Coleman, M., Woods, P. 2008. Evaluation of tropospheric and stratospheric ozone trends over Western Europe from ground-based FTIR network observations. Atmospheric Chemistry and Physics 8, 6865-6886. Special Issue 'Results from the European project UFTIR, Time series of Upper Free Troposphere observations from a European ground-based FTIR network.

Villani, P., Picard, D., Marchand, N., Laj, P., 2007. Design and validation of a 6volatility tandem differential mobility analyzer (VTDMA). Aerosol Science and Technology 41, 898-906.

Villani, P., Picard, D., Michaud, V., Laj, P., Wiedensohler, A., 2008. Design and validation of a volatility hygroscopic tandem differential mobility analyzer (VHTDMA) to characterize the relationships between the thermal and hygroscopic properties of atmospheric aerosol particles. Aerosol Science and Technology 42, 729-741.

Virkkula, A., Ahlquist, N.C., Covert, D.S., Arnott, W.P., Sheridan, P.J., Quinn, P.K., Coffmann, D.J., 2005. Modification, calibration and a field test of an instrument for measuring light absorption by particles. Aerosol Science and Technology 39 68-83.

Voisin, D., Smith, J.N., Sakurai, H., McMurry, P.H., Eisele, F.L., 2003. Thermal desorption chemical ionization mass spectrometer for ultrafine particle chemical composition. Aerosol Science and Technology 37, 471-475.

Volkamer, R., Jimenez, J.L., Martini, F.S., Dzepina, K., Zhang, Q., Salcedo, D., Molina, L.T., Worsnop, D.R., Molina, M.J., 2006. Secondary organic aerosol formation from anthropogenic air pollution: rapid and higher than expected. Geophysical Research Letters 33, L17811.

Volz-Thomas, A., the IAGOS Team, 2007. In-service aircraft for global observations, the future. International Global Atmospheric Chemistry (IGAC) Newsletter. von Hoyningen-Huene, W., Kokhanovsky, A.A., Wuttke, M.W., Buchwitz, M., Noël, S., Gerilowski, K., Burrows, J.P., Latter, B., Siddans, R., Kerridge, B.J., 2007. Validation of SCIAMACHY top-of-atmosphere reflectance for aerosol remote sensing using MERIS L1 data. Atmospheric Chemistry and Physics 7, 97-106.

Vrekoussis, M., Kanakidou, M., Mihalopoulos, N., Crutzen, P.J., Lelieveld, J., Perner, D. Berresheim, H., Baboukas, E., 2004. Role of the $\mathrm{NO}_{3}$ radicals in oxidation processes in the eastern Mediterranean troposphere during the MINOS campaign. Atmospheric Chemistry and Physics 4, 169-182.

Vrekoussis, M., Mihalopoulos, N., Gerasopoulos, E., Kanakidou, M., Crutzen, P.J., Lelieveld, J., 2007. Two-years of $\mathrm{NO}_{3}$ radical observations in the boundary layer over the Eastern Mediterranean. Atmospheric Chemistry and Physics 7, 315-327.

Wagner, T., Dix, B., Friedeburg, C.v., Frieß, U., Sanghavi, S., Sinreich, R., Platt, U., 2004. MAX-DOAS $\mathrm{O}_{4}$ measurements - a new technique to derive information on atmospheric aerosols. (I) Principles and information content. Journal of Geophysical Research 109, D22205.

Wagner, T., Platt, U., 1998. Satellite mapping of enhanced BrO concentrations in the troposphere. Nature 395, 486-490.

Wal, R.L.V., Ticich, T.M., West, J.R., Householder, P.A., 1999. Trace metal detection by laser-induced breakdown spectroscopy. Applied Spectroscopy 53 (10), 388A405A, 1226-1236.

Wandinger, U., Müller, D., Böckmann, C., Althausen, D., Matthias, V., Bösenberg, J., Weiß, V., Fiebig, M., Wendisch, M., Stohl, A., Ansmann, A., 2002. Optical and microphysical characterization of biomass-burning and industrial-pollution aerosols from multiwavelength lidar and aircraft measurements. Journal of Geophysical Research 107, D21.

Wang, J., McNeill, V.F., Collins, D.R., Flagan, R.C., 2002. Fast mixing condensation nucleus counter: application to rapid scanning differential mobility analyzer measurements. Aerosol Science and Technology 36, 678-689.

Wängberg, I., Barnes, I., Becker, K.H., 1997. Product and mechanistic study of the reaction of $\mathrm{NO}_{3}$ radicals with $\alpha$-pinene. Environmental Science and Technology 31, 2130-2135

Warneke, T., Yang, Z., Olsen, S., Korner, S., Notholt, J., Toon, G.C., Velazco, V., Schulz, A., Schrems, O. 2005. Seasonal and latitudinal variations of column averaged volume-mixing ratios of atmospheric $\mathrm{CO}_{2}$. Geophysical Research Letters 32, L03808.

Warneke, C., de Gouw, J.A., Lovejoy, E.R., Murphy, P.C., Kuster, W.C., Fall, R., 2005 Development of proton-transfer ion trap-mass spectrometry: on-line detection and identification of volatile organic compounds in air. Journal of the American Society for Mass Spectrometry 16, 1316-1324.

Warneke, C., Kato, S., De Gouw, J.A., Goldan, P.D., Kuster, W.C., Shao, M., Lovejoy, E.R. Fall, R., Fehsenfeld, F.C., 2005. Online volatile organic compound measurements using a newly developed proton-transfer ion-trap mass spectrometry instrument during New England Air Quality Study-Intercontinental Transport and Chemical Transformation 2004: performance, intercomparison, and compound identification. Environmental Science and Technology 39, 5390-5397.

Warren, B., Song, C., Cocker, D.R., 2008. Light intensity and light source influence on secondary organic aerosol formation for the m-xylene/ $\mathrm{NO}_{\mathrm{x}}$ photooxidation system. Environmental Science and Technology 42, 5461-5466.

Washenfelder, R., 2006. Column Abundances of Carbon Dioxide and Methane Retrieved from Ground-based Near-infrared Solar Spectra. Ph.D. thesis. Caltech University.

Watson, I.M., Realmuto, V.J., Rose, W.I., Prata, A.J., Bluth, G.J.S., Gu, Y., Bader, C.E., $\mathrm{Yu}, \mathrm{T}$., 2004. Thermal infrared remote sensing of volcanic emissions using the moderate resolution imaging spectroradiometer. Journal of Volcanology and Geothermal Research 135, 75-89.

Weatherhead, E.C., Reinsel, G.C., Tiao, G.C., Meng, X.L., Choi, D.S., Cheang, W.K., Keller, T., DeLuisi, J., Wuebbles, D.J., Kerr, J.B., Miller, A.J., Oltmans, S.J., Frederick, J.E., 1998. Factors affecting the detection of trends: statistical considerations and applications to environmental data. Journal of Geophysical Research - Atmospheres 103, 17149-17161.

Weber, R.J., Stolzenburg, M.R., Pandis, S.N., McMurry, P.H., 1998. Inversion of ultrafine condensation nucleus counter pulse height distributions to obtain nanoparticle (similar to 3-10 nm) size distributions. Journal of Aerosol Science 29, 601-615.

Weber, R.J., Orsini, D., Daun, Y., Lee, Y.N., Klotz, P.J., Brechtel, F., 2001. A particle-intoliquid collector for rapid measurement of aerosol bulk chemical composition. Aerosol Science and Technology 35, 718-727.

Weber, R.J., Sullivan, A.P., Peltier, R.E., Russell, A., Yan, B., Zheng, M., de Gouw, J., Warneke, C., Brock, C., Holloway, J.S., Atlas, E.L., Edgerton, E., 2007. A study of secondary organic aerosol formation in the anthropogenic-influenced southeastern United States. Journal of Geophysical Research 112, D13302.

Wehner, B., Philippin, S., Wiedensohler, A., 2002. Design and calibration of a thermodenuder with an improved heating unit to measure the size-dependent volatile fraction of aerosol particles. Journal of Aerosol Science 33, 1087-1093.

Wehrli, C., 2000. Calibrations of filter radiometers for determination of atmospheric optical depth. Metrologia 37, 419-422.

Weigelt, A., Hermann, M., van Velthoven, P.F.J., Brenninkmeijer, C.A.M., Schlaf, G., Zahn, A., Wiedensohler, A., 2009. Influence of clouds on aerosol particle number concentrations in the upper troposphere. Journal of Geophysical Research 114 D01204.

Weimer, S., Mohr, C., Richter, R., Mohr, M., Prevot, A.S.H., Baltensperger, U., 2009. Highly time and spatially resolved measurements of aerosol size distributions with an FMPS in an Alpine valley. Atmospheric Environment 43, 624-630. 
Weingartner, E., Saathoff, H., Schnaiter, M., Streit, N., Bitnar, B., Baltensperger, U., 2003. Absorption of light by soot particles: determination of the absorption by means of aethalometers. Journal of Aerosol Science 34, 1445-1463.

Weitkamp, E.A., Sage, A.M., Pierce, J.R., Donahue, N.M., Robinson, A.L., 2007. Organic aerosol formation from photochemical oxidation of diesel exhaust in a smog chamber. Environmental Science and Technology 41, 6969-6975.

Wen, G., Marshak, A., Cahalan, R.F., 2006. Impact of 3D clouds on clear sky reflectance and aerosol retrieval in a biomass burning region of Brazil. IEEE Transactions on Geoscience and Remote Sensing 3, 169-172.

Wespes, C., Hurtmans, D., Herbin, H., Barret, B., Turquety, S., Hadji-Lazaro, J., Clerbaux, C., Coheur, P.F., 2007. First global distributions of nitric acid in the troposphere and the stratosphere derived from infrared satellite measurements. Journal of Geophysical Research - Atmospheres 112, D13311.

Wex, H., Kiselev, A., Stratmann, F., Zoboki, J., Brechtel, F., 2005. Measured and modeled equilibrium sizes of $\mathrm{NaCl}$ and $\left(\mathrm{NH}_{4}\right)_{2} \mathrm{SO}_{4}$ particles at relative humidities up to $99.1 \%$. Journal of Geophysical Research 110.

Wex, H., Ziese, M., Kiselev, A., 2007. Deliquescence and hygroscopic growth of succinic acid particles measured with LACIS. Geophysical Research Letters 34, L17810.

Williams, B.J., Goldstein, A.H., Kreisberg, N.M., Hering, S.V., 2006. An in situ instrument for speciated organic composition of atmospheric aerosols: thermal desorption aerosol GC/MS-FID (TAG). Aerosol Science and Technology 40, 627-638.

Williams, B.J., Goldstein, A.H., Millet, D.B., Holzinger, R., Kreisberg, N.M., Hering, S.V., White, A.B., Worsnop, D.R., Allan, J.D., Jimenez, J.L., 2007. Chemical speciation of organic aerosol during the international consortium for atmospheric research on transport and transformation 2004: results from in situ measurements. Journal of Geophysical Research - Atmospheres 112, D10S26.

Wilson, J., Lafleur, B., Hilbert, H., Seebaugh, W., Fox, J., Gesler, D., Brock, C., Huebert, B., Mullen, J., 2004. Function and performance of a low turbulence inlet for sampling supermicron particles from aircraft platforms. Aerosol Science and Technology 38, 790-802.

Winker, D.M., Hunt, W.H., McGill, M.J., 2007. Initial performance assessment of CALIOP. Geophysical Research Letters 34, L19803.

Winterholler, B., Hoppe, P., Foley, S., Andreae, M.O., 2008. Sulfur isotope ratio measurements of individual sulfate particles by NanoSIMS. International Journal of Mass Spectrometry 272, 63-77.

Wise, M.E., Garland, R.M., Tolbert, M.A., 2004. Ice nucleation in internally mixed ammonium sulfate/dicarboxylic acid particles. Journal of Geophysical Research 109, D19203.

Wisthaler, A., Apel, E.C., Bossmeyer, J., Hansel, A., Junkermann, W., Koppmann, R., Meier, R., Müller, K., Solomon, S.J., Steinbrecher, R., Tillmann, R., Brauers, T., 2008. Technical note: intercomparison of formaldehyde measurements at the atmosphere simulation chamber. Atmospheric Chemistry and Physics 8, 2189-2200.

Wittrock, F., Oetjen, H., Richter, A., Fietkau, S., Medeke, T., Rozanov, A., Burrows, J.P., 2004. MAX-DOAS measurements of atmospheric trace gases in Ny-Ålesund radiative transfer studies and their application. Atmospheric Chemistry and Physics 4, 955-966.

Wittrock, F., Richter, A., Oetjen, H., Burrows, J.P., Kanakidou, M., Myriokefalitakis, S., Volkamer, R., Beirle, S., Platt, U., Wagner, T., 2006. Simultaneous global observations of glyoxal and formaldehyde from space. Geophysical Research Letters 33, L16804.

WMO, 2007. WMO Global Atmosphere Watch (GAW) Strategic Plan (2008-2015). GAW Report No. 172 (WMO TD NO. 1384). World Meteorological Organization, Geneva, Switzerland.

Wood, E.C., Wooldridge, P.J., Freese, J.H., Albrecht, T., Cohen, R.C., 2003. Prototype for in situ detection of atmospheric $\mathrm{NO}_{3}$ and $\mathrm{N}_{2} \mathrm{O}_{5}$ via laser-induced fluorescence. Environmental Science and Technology 37, 5732-5738.

Worden, H.M., Logan, J.A., Worden, J.R., Beer, R., Bowman, K., Clough, S.A., Eldering, A., Fisher, B.M., Gunson, M.R., Herman, R.L., Kulawik, S.S., Lampel, M.C. Luo, M., Megretskaia, I.A., Osterman, G.B., Shephard, M.W., 2007. Comparisons of tropospheric emission spectrometer (TES) ozone profiles to ozonesondes: methods and initial results. Journal of Geophysical Research - Atmospheres 112, D03309.

Worden, J., Liu, X., Bowman, K., Chance, K., Beer, R., Eldering, A., Gunson, M., Worden, H., 2007. Improved tropospheric ozone profiles using OMI and TES radiances. Geophysical Research Letters 34, L01809.

Worden, H.M., Bowman, K.W., Worden, J.R., Eldering, A., Beer, R., 2008. Satellite measurements of the clear-sky greenhouse effect from tropospheric ozone. Nature Geoscience 1, 305-308.

Wyche, K.P., Monks, P.S., Ellis, A.M., Cordell, R.L., Parke, A.E., Whyte, C. Metzger, A.r.J., Dommen, J., Duplissy, J., Prevot, A.S.H., Baltensperger, U., Rickard, A.R., Wulfert, F., 2009. Gas phase precursors to anthropogenic secondary organic aerosol: detailed observations of 1,3,5-trimethylbenzene photooxidation. Atmospheric Chemistry and Physics 9, 635-665.

Xiong, X.Z., Barnet, C., Maddy, E., Sweeney, C., Liu, X.P., Zhou, L.H., Goldberg, M., 2008. Characterization and validation of methane products from the Atmospheric Infrared Sounder (AIRS). Journal of Geophysical Research - Biogeosciences 113 , G00A01.

Xu, X., Williams, J., Plass-Dülmer, C., Berresheim, H., Salisbury, G., Lange, L., Lelieveld, J., 2003. GC $\times$ GC measurements of C7-C11 aromatic and n-alkane hydrocarbons on Crete, in air from Eastern Europe during the MINOS campaign. Atmospheric Chemistry and Physics 3, 1461-1475.
Xue, H., Moyle, A., Magee, N., Harrington, J., Lamb, D., 2005. Experimental studies of droplet evaporation kinetics: validation of models for binary and ternary aqueous solutions. Journal of the Atmospheric Sciences 62 (12), 4310-4326. doi:10.1175/JAS3623.1

Yang, Z.H., Toon, G.C., Margolis, J.S., Wennberg, P.O., 2002. Atmospheric $\mathrm{CO}_{2}$ retrieved from ground-based near IR solar spectra. Geophysical Research Letters 29, 1339.

Yang, K., Krotkov, N.A., Krueger, A.J., Carn, S.A., Bhartia, P.K., Levelt, P.F., 2007. Retrieval of large volcanic $\mathrm{SO}_{2}$ columns from the Aura ozone monitoring instrument: comparison and limitations. Journal of Geophysical Research Atmospheres 112, D24S43.

Yeatman, S.G., Spokes, L.J., Dennis, P.F., Jickells, T.D., 2001. Can the study of nitrogen isotopic composition in size-segregated aerosol nitrate and ammonium be used to investigate atmospheric processing mechanisms? Atmospheric Environment 35, 1337-1345.

Yu, J., Cocker, D.R., Griffin, R.J., Flagan, R.C., Seinfeld, J.H., 1999. Gas-phase ozone oxidation of monoterpenes: gaseous and particulate products. Journal of Atmospheric Chemistry 34, 207-258.

Yu, H.B., Dickinson, R.E., Chin, M., Kaufman, Y.J., Holben, B.N., Geogdzhayev, I.V. Mishchenko, M.I., 2003. Annual cycle of global distributions of aerosol optica depth from integration of MODIS retrievals and GOCART model simulations. Journal of Geophysical Research - Atmospheres 108, 4128.

Zardini, A.A., Krieger, U.K., Marcolli, C., 2006. White light Mie resonance spectroscopy used to measure very low vapor pressures of substances in aqueous solution aerosol particles. Optics Express 14, 6951-6962.

Zardini, A.A., Sjogren, S., Marcolli, C., Krieger, U.K., Gysel, M., Weingartner, E. Baltensperger, U., Peter, T., 2008. A combined particle trap/HTDMA hygroscopicity study of mixed inorganic/organic aerosol particles. Atmospheric Chemistry and Physics 8, 5589-5601.

Zbinden, R.M., Cammas, J.-P., Thouret, V., Nédélec, P., Karcher, F., Simon, P., 2006. Midlatitude tropospheric ozone columns from the MOZAIC program: climatology and interannual variability. Atmospheric Chemistry and Physics 6, 1053-1073.

Zelenyuk, A., Imre, D., 2005. Single particle laser ablation time-of-flight mass spectrometer: an introduction to SPLAT. Aerosol Science and Technology 39, 554-568.

Zellweger, C., Ammann, M., Hofer, P., Baltensperger, U., 1999. $\mathrm{NO}_{\mathrm{y}}$ speciation with a combined wet effluent diffusion denuder-aerosol collector coupled to ion chromatography. Atmospheric Environment 33, 1131-1140.

Zhang, X.F., Smith, K.A., Worsnop, D.R., Jimenez, J., Jayne, J.T., Kolb, C.E., 2002. A numerical characterization of particle beam collimation by an aerodynamic lens-nozzle system: part I. An individual lens or nozzle. Aerosol Science and Technology 36, 617-631.

Zhang, X.F., Smith, K.A., Worsnop, D.R., Jimenez, J.L., Jayne, J.T., Kolb, C.E., Morris, J., Davidovits, P., 2004. Numerical characterization of particle beam collimation: part II - integrated aerodynamic-lens-nozzle system. Aerosol Science and Technology 38, 619-638.

Zhang, Q., Alfarra, M.R., Worsnop, D.R., Allan, J.D., Coe, H., Canagaratna, M.R. Jimenez, J.L., 2005. Deconvolution and quantification of hydrocarbon-like and oxygenated organic aerosols based on aerosol mass spectrometry. Environmental Science and Technology 39, 4938-4952.

Zhang, L., Jacob, D.., Bowman, K.W., Logan, J.A., Turquety, S., Hudman, R.C., Li, Q.B. Beer, R., Worden, H.M., Worden, J.R., Rinsland, C.P., Kulawik, S.S., Lampel, M.C. Shephard, M.W., Fisher, B.M., Eldering, A., Avery, M.A., 2006. Ozone-CO correlations determined by the TES satellite instrument in continental outflow regions. Geophysical Research Letters 33, L18804.

Zhang, Q., Jimenez, J.L., Canagaratna, M.R., Allan, J.D., Coe, H., Ulbrich, I., Alfarra, M.R., Takami, A., Middlebrook, A.M., Sun, Y.L., Dzepina, K., Dunlea, E., Docherty, K., DeCarlo, P.F., Salcedo, D., Onasch, T., Jayne, J.T., Miyoshi, T., Shimono, A., Hatakeyama, S., Takegawa, N., Kondo, Y., Schneider, J., Drewnick, F., Borrmann, S., Weimer, S., Demerjian, K., Williams, P., Bower, K., Bahreini, R. Cottrell, L., Griffin, R.J., Rautiainen, J., Sun, J.Y., Zhang, Y.M., Worsnop, D.R., 2007. Ubiquity and dominance of oxygenated species in organic aerosols in anthropogenically-influenced Northern Hemisphere midlatitudes. Geophysical Research Letters 34, L13801.

Ziemke, J.R., Chandra, S., Bhartia, P.K., 2001. "Cloud slicing”: a new technique to derive upper tropospheric ozone from satellite measurements. Journal of Geophysical Research - Atmospheres 106, 9853-9867.

Zientara, M., Jakubczyk, D., Derkachov, G., Kolwas, K., Kolwas, M., 2005. Simultaneous determination of mass and thermal accommodation coeincients from temporal evolution of an evaporating water microdroplet. Journal of Physics D: Applied Physics 38, 1978-1983.

Zobrist, B., Marcolli, C., Koop, T., Luo, B.P., Murphy, D.M., Lohmann, U., Zardini, A.A Krieger, U.K., Corti, T., Cziczo, D.J., Fueglistaler, S., Hudson, P.K., Thomson, D.S. Peter, T., 2006. Oxalic acid as a heterogeneous ice nucleus in the upper troposphere and its indirect aerosol effect. Atmospheric Chemistry and Physics 6 , 3115-3129.

Zobrist, B., Marcolli, C., Pedernera, D.A., Koop, T., 2008. Do atmospheric aerosols form glasses? Atmospheric Chemistry and Physics 8, 5221-5244.

Zuberi, B., Bertram, A.K., Cassa, C.A., Molina, L.T., Molina, M.J., 2002. Heterogeneous nucleation of ice in $\left(\mathrm{NH}_{4}\right)_{2} \mathrm{SO}_{4}-\mathrm{H}_{2} \mathrm{O}$ particles with mineral dust immersions. Geophysical Research Letters 29, 1504.

Zuberi, B., Johnson, K.S., de Foy, B., 2006. Aerosol composition and source apportionment in the Mexico City Metropolitan Area with PIXE/PESA/STIM and multivariate analysis. Atmospheric Chemistry and Physics 6, 4591-4600. 